\title{
Photolytischer Käfigeffekt von Dihalomethanen in überkritischen Lösungsmitteln
}

\author{
Dissertation \\ zur Erlangung des Doktorgrades \\ der Mathematisch-Naturwissenschaftlichen Fakultäten \\ der Georg-August-Universität zu Göttingen
}

vorgelegt von

Jochen Zerbs

aus Fulda

Göttingen 2005 
D7

Referent: Prof. Dr. J. Troe

Korreferent: Prof. Dr. D. Schwarzer

Tag der mündlichen Prüfung: 02.11.2005 
"Alles ist so, wie es ist, weil es so ist."

Die Goldenen Zitronen, Auf der Unterseite 



\section{Danksagung}

Herrn Prof. Jürgen Troe danke ich für seine stetige und wohlwollende Förderung dieser Arbeit. Die hervorragende Arbeitsatmosphäre in seiner Gruppe hat mir immer sehr viel Freude bereitet und dadurch sehr zum Gelingen dieser Arbeit beigetragen.

Herrn Prof. Jörg Schroeder danke ich für seine fortwährende Begeisterung für das Projekt. Die Diskussionen mit ihm haben mir immer sehr geholfen und neue Ideen und Erkenntnisse gebracht.

Zudem danke ich Prof. Dirk Schwarzer für wertvolle Diskussionen und Anregungen sowie die freundliche Übernahme des Korreferats.

Ich danke ganz herzlich Sascha Kandratsenka für die Durchführung der MD-Simulationen. Ohne seine radialen Verteilungsfunktionen wäre ich wahrscheinlich niemals auf die Idee gekommen, meine Daten quadratisch anzupassen.

Mein ganz besonderer Dank gilt meinen engsten Mitarbeitern, die mich durch dieses auBerordentlich spannende Projekt begleitet haben. Dank sei Christian Grimm, der mich trotz allem damals in der Gruppe aufgenommen hat und mit dem ich die Diiodmethan Messungen angefangen habe. Für Toleranz gegenüber schwermütiger Musik bedanke mich auch bei meinem „Erben“ Philipp Wagener, bei dem ich mir sicher sein kann, dass das Labor 247 bei ihm in guten Händen liegt. Für die Einführung in die wunderbare Welt des Weines bedanke ich mich bei Tobias Steinel, der mich damals in mein erstes Experiment eingearbeitet hat und das ich dann leider zerlegt habe (es tut mir leid...). Ein weiteres großes Dankeschön geht nach Amsterdam zu Matthias Kling, dem wohl enthusiastischsten Wissenschaftler, den ich kennenlernen durfte. Mithin danke ich „el Professore“ Christian Müller für außerordentlich fruchtbare wissenschaftliche, philosophische und politische Diskussionen und seinen wertvollen, zuweilen aber auch besserwisserisch vorgetragenem Ratschlage, doch mal endlich irgendwelche Rechnungen zu machen. In ihm habe ich den einzigen Menschen gefunden, der sich von mir musikalisch beeinflussen ließ - zum Guten wie zum Bösen. Nochmal ein riesiges Danke an die gesamte Schroeder Gruppe, lasst Euch von niemandem sagen, dass Ihr nicht die Besten seid!

An dieser Stelle möchte ich meinen Korrekturlesern danken, die es geschafft haben, die kleineren und größeren Fehler auszumerzen: Philipp Wagener, Matthias Kling, Jörg Hahn, Frauke Schröder, Marco Seidel und Christian Müller.

Ich tue allen Mitarbeitern der Werkstätten unrecht, dass ich sie an solch später Stelle danke, weil ohne ihren immer schnellen und überaus hilfsbereiten Einsatz mein Experiment schon längsten in alle Einzelteile zerfallen wäre. Und ich tue wiederum unrecht, wenn ich namentlich nur die Leiter der mechanischen und elektronischen Werkstätten, die Herren Volker Meier und Andreas Knorr, nenne. Außerdem danke ich Milo, der guten Seele des Instituts.

Ich möchte mich hier noch bei dem Rest der Abteilung für das ganz besondere Abteilungsklima bedanken: den Administratoren Oliver und Björn, Britta, Kawon und Thomas, 
Anatoli (danke für mehrmalige Nachhilfe in Mathematik), Ravi, Heiko und Matthias, Jaane, Esteban, Chang, Reinhard (dem ich hiermit für kleinere und größere Wunder danke) und denen, die schon lange nicht mehr da sind: Christoph, Tim, Uwe, Marco (der für mich immer irgendwo zwischen Pur und Slayer stehen wird), Sven (bei dem ich mich hiermit entschuldigen möchte, dass der Wind nie geweht hat) und Rosalin.

Und ich möchte an dieser Stelle meinem Freundeskreis danken, die zwar nicht unmittelbar am meiner Arbeit mitgewirkt haben, die aber doch immer einen positiven Einfluss auf mich hatten: André, dem bayrischten aller Göttinger, Nicole (danke für diverse Analysen), den besten Tauchpartnern aller Zeiten: Nicola, Carmen und Moritz, dem besten Tauchlehrer aller Zeiten: Clemens, Hans und Holger für schlechtere und bessere Zeiten in unserer Küche, Ilga (der einzige Mensch, der meine Besessenheit von NC teilt), Alessandra, Ruzica und schließlich Ulrich. Und natürlich sei da noch die Doppelkopfrunde vom MPI erwähnt: Frauke, Christian und Michael.

Meiner Familie danke ich dafür, dass sie mich immer unterstützt und gefördert hat.

Danke Nick Cave für wunderbar leidenschaftliche Musik. 


\section{Inhaltsverzeichnis}

\begin{tabular}{lll}
\hline 1 & Einleitung & 1
\end{tabular}

2 Grundlagen 3

2.1 Der Käfigeffekt des Iod-Moleküls . . . . . . . . . . . . . . . . . . . . . . 3

2.1.1 Das Diffusionsmodell nach Otto, Schroeder und Troe . . . . . . . . . 4

$2.1 .2 \quad$ Dynamische Studien . . . . . . . . . . . . . . . . . . 6

2.1.3 Kinematischer Käfigeffekt . . . . . . . . . . . . . . . . . . . . . . . . . . 6

2.2 Der Käfigeffekt des Diiodmethan-Moleküls . . . . . . . . . . . . . . . . . . . 8

$2.2 .1 \quad$ Spektroskopie und Dissoziationsdynamik . . . . . . . . . . . . . . . . . . . 8

$2.2 .2 \quad \mathrm{CH}_{2} \mathrm{I}_{2}$ in der kondensierten Phase . . . . . . . . . . . . . . . . . . . 11

2.3 Der Käfigeffekt des Bromiodmethan-Moleküls . . . . . . . . . . . . . . . . . 14

$2.3 .1 \quad$ Spektroskopie und Dissoziationsdynamik . . . . . . . . . . . . . . . . 14

$2.3 .2 \mathrm{CH}_{2} \mathrm{BrI}$ in der kondensierten Phase . . . . . . . . . . . . . . . . . . . 14

2.4 Solvatation in überkritischen Fluiden . . . . . . . . . . . . . . . . . . . 15

\begin{tabular}{lll}
\hline & Experimentelle Technik & 19
\end{tabular}

3.1 Wahl der Methode . . . . . . . . . . . . . . . . . . . . . . . . . . . . . . . . 19

3.2 Das Lasersystem im Überblick . . . . . . . . . . . . . . . . . . . . . . . . . . 19

3.3 Die Komponenten des Lasersystems . . . . . . . . . . . . . . . . . . . . . . . 20

$3.3 .1 \quad$ CPA-2001 . . . . . . . . . . . . . . . . . . . . . . . 20

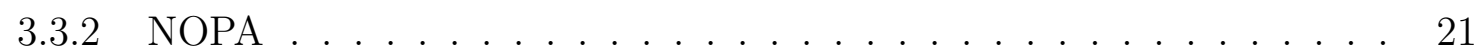

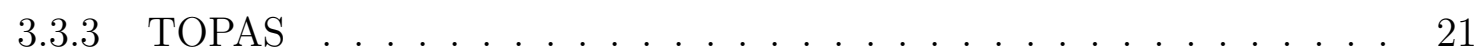

3.3 .4 Pump-Probe-Interferometer . . . . . . . . . . . . . . . 22

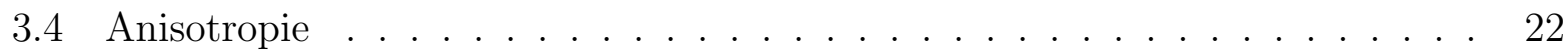

3.5 Anpassung der Daten . . . . . . . . . . . . . . . . . . . . . . . . . . 24

3.6 Verwendete Chemikalien . . . . . . . . . . . . . . . . . . . . . . . . . . . . . 25

\begin{tabular}{|lll}
\hline 4 & Experimentelle Ergebnisse & 27
\end{tabular}

4.1 Stationäre Absorptionsspektren . . . . . . . . . . . . . . . . . . . . . . . 27

4.2 Zeitaufgelöste Absorptionsmessungen an $\mathrm{CH}_{2} \mathrm{I}_{2} \quad \ldots \ldots \ldots$. . . . . . . . . . . . . . 27

$4.2 .1 \quad$ Probewellenlängenabhängigkeit . . . . . . . . . . . . . . . . . . . 30

4.2.2 $\quad$ Dichteabhängigkeit . . . . . . . . . . . . . . . . . . . . . . . . . . . 32

$4.2 .3 \quad$ Lösungsmittelabhängigkeit . . . . . . . . . . . . . . . . . . . . . . . . . 39

$4.2 .4 \quad$ Anregungswellenlängenabhängigkeit . . . . . . . . . . . . . . . . . . . . . . . . . . 42

4.3 Zeitaufgelöste Absorptionsmessungen an $\mathrm{CH}_{2} \mathrm{BrI}$. . . . . . . . . . . . . . . . . . 45

4.4 Zeitaufgelöste Anisotropiemessungen . . . . . . . . . . . . . . . . . . . . . 47 
5 Molekulardynamische Simulationen $\quad 51$

5.1 Verwendete Potentiale . . . . . . . . . . . . . . . . . . . . . 51

5.2 Radiale Verteilungsfunktion von $\mathrm{CH}_{2} \mathrm{I}_{2}$ in Xe . . . . . . . . . . . . . . . 53

5.3 Radiale Verteilungsfunktion von $\mathrm{CH}_{2} \mathrm{I}_{2}$ in $\mathrm{CO}_{2} \ldots \ldots \ldots$. . . . . . . . . . . . . 53

5.4 Radiale Verteilungsfunktion von $\mathrm{CH}_{2} \mathrm{I}_{2}$ in $\mathrm{C}_{2} \mathrm{H}_{6} \ldots \ldots \ldots \ldots$. . . . . . . 56

5.5 Radiale Verteilungsfunktion von $\mathrm{CH}_{2} \mathrm{I}_{2}$ in $\mathrm{CHF}_{3} \ldots \ldots \ldots$. . . . . . . . . 58

5.6 Zusammenfassung . . . . . . . . . . . . . . . . . . . . 58

\begin{tabular}{|lll}
\hline $\mathbf{6}$ & Diskussion & $\mathbf{6 1}$
\end{tabular}

6.1 Zuordnung im Kurzzeitbereich . . . . . . . . . . . . . . . . . . . . . . . . 61

$6.1 .1 \quad$ Franck-Condon Region des angeregten $\mathrm{CH}_{2} \mathrm{I}_{2}$. . . . . . . . . . . . . . 61

6.1 .2 Direkte Isomerbildung . . . . . . . . . . . . . . . . . . . . . . . 61

$6.1 .3 \quad \mathrm{CH}_{2} \mathrm{I}$-Radikal $\ldots \ldots \ldots \ldots$. . . . . . . . . . . . . . . . . . . . 62

6.1 .4 Geminale Rekombination zum Muttermolekül . . . . . . . . . . . . . 64

$6.1 .5 \quad$ Ladungstransferkomplex . . . . . . . . . . . . . . . . . . . 65

6.1 .6 Orientierungsrelaxation . . . . . . . . . . . . . . . . . . . 65

6.1 .7 Zusammenfassung . . . . . . . . . . . . . . . . . . . . . . . . . . . . . . . . . . . 67

6.2 Quantenausbeuten . . . . . . . . . . . . . . . . . 67

6.2 .1 Lösungsmittelabhängigkeit . . . . . . . . . . . . . . . . . . . . 67

$6.2 .2 \quad$ Dichteabhängigkeit . . . . . . . . . . . . . . . . . . . . . . . 68

6.3 Bildungsgeschwindigkeit . . . . . . . . . . . . . . . . . . . . . . . 74

6.3 .1 Lösungsmittelabhängigkeit . . . . . . . . . . . . . . . . . . . . . . . . . . . . . . . . . . . . . . 75

6.3 .2 Dichteabhängigkeit . . . . . . . . . . . . . . . . . . . 76

$6.3 .3 \quad$ Probewellenlängenabhängigkeit . . . . . . . . . . . . . . . . . . . . . 79

$6.3 .4 \quad$ Anregungswellenlängenabhängigkeit . . . . . . . . . . . . . . . . . . . 82

6.4 Zusammenfassung . . . . . . . . . . . . . . . . . . 84

\begin{tabular}{ll}
\hline Anhang & $\mathbf{8 5}$
\end{tabular}

A.1 Kritische Daten der verwendeten Lösungsmittel . . . . . . . . . . . . . . . . 85

A.2 Zeitaufgelöste Absorptionsspektren in $\mathrm{Xe}, \mathrm{C}_{2} \mathrm{H}_{6}$ und $\mathrm{CHF}_{3} \ldots \ldots$. . . . . . 85

A.3 $\quad$ Sulzer-Wieland Modell zur Abschätzung der Radikalabsorption . . . . . . . . . 89

\begin{tabular}{llll}
\hline A.4 & Zeitaufgelöste Absorptionszeitprofile in $\mathrm{Xe}, \mathrm{C}_{2} \mathrm{H}_{6}$ und $\mathrm{CHF}_{3}$
\end{tabular}$\ldots$. . . . . . . . 89

A.5 Korrelation der Quantenausbeuten mit den lokalen Dichten in $\mathrm{Xe}_{2} \mathrm{C}_{2} \mathrm{H}_{6}$ und

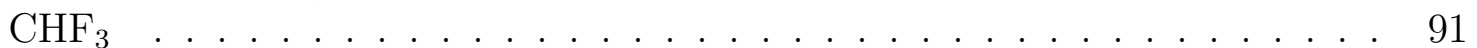

\begin{tabular}{ll}
\hline Literaturverzeichnis & 93
\end{tabular} 


\section{Einleitung}

Eine Vielzahl chemischer Reaktionen wird in Lösung durchgeführt. Dabei kann das Lösungsmittel die Reaktionspartner nicht nur komplexieren und mit einer Solvathülle umgeben, sondern auch direkten Einfluss auf die Reaktion nehmen, in dem es das Diffusionsverhalten der Reaktanden kontrolliert, die Aktivierungsenergien modifiziert oder die Potentialflächen, auf denen die Dynamik abläuft, verändert. Um solche Einflüsse der Lösungsmittelumgebung zu untersuchen, wurde für diese Arbeit eine möglichst einfache Reaktion gewählt: die Photodissoziation und anschließende Isomerisierung von Dihalomethanen. In der Gasphase ist die Photodissoziationsquantenausbeute eins, während in Lösung die Isomerisierung als Konkurrenzprozess auftritt und die Quantenausbeute dementsprechend kleiner wird. Diese Verringerung der Photodissoziationsquantenausbeute auf Werte kleiner eins beim Übergang von isolierten Bedingungen auf nicht isolierte Bedingungen wird als photolytischer Käfigeffekt bezeichnet [1].

Der photolytische Käfigeffekt wird seit den 1930er Jahren am Beispiel des Iodmoleküls untersucht, in dieser Arbeit werden die Untersuchungen auf die größeren Systeme Diiodmethan und Bromiodmethan ausgeweitet. Als Lösungsmittel wurden überkritische Fluide gewählt, weil sie auf Grund ihrer Inhomogenitäten eine hohe Kompressibiliät aufweisen und sich so durch Änderung des Drucks die Lösungsmitteleigenschaften wie z.B. Dichte, Polarität und Viskosität über einen weiten Bereich in beliebiger Schrittweite variieren lassen. Als experimentelle Technik wurde die Pump-Probe Absorptionsspektroskopie mit Femtosekundenzeitauflösung verwendet. Die erhaltenen Absorptionszeitprofile stimmen mit den aus der Literatur bekannten Transienten überein und konnten in dieser Arbeit erstmals auf ihre Druck- und damit Dichteabhängigkeit untersucht werden. Ziel dieser Methode ist es, einen funktionellen Zusammenhang zwischen der Reaktionskinetik und den makroskopischen Eigenschaften des Lösungsmittels zu finden.

Die vorliegende Arbeit ist wie folgt gegliedert: Im zweiten Kapitel wird zunächst auf den Käfigeffekt des Iodmoleküls eingegangen. Dabei werden insbesonders Diffusionsmodelle und der kinematische Käfigeffekt gegenübergestellt. Anschließend wird der derzeitige Stand der Forschung zur Dissoziation von Diiodmethan und Bromiodmethan in der Gasphase sowie zur Isomerisierung in der flüssigen Phase zusammengefasst. Das Kapitel schließt mit einer Ausführung über die Eigenschaften überkritischer Fluide ab. In Kapitel 3 wird das verwendete Laser- und das Hochdrucksystem beschrieben. Die gemessenen Absorptionszeitprofile werden im vierten Kapitel dargestellt und ihre Dichte- und Wellenlängenabhängigkeit wird ausführlich beschrieben. Zudem wurden zur Charakterisierung der im Kurzzeitbereich absorbierenden Spezies dichteabhängige Anisotropiemessungen durchgeführt. Um Informationen über die Struktur der Solvathülle um das Diiodmethan und ihre Dichte- sowie Lösungsmittelabhängigkeit zu erlangen, wurden klassische molekulardynamische Simulationen durchgeführt. Die verwendeten Potentiale und die erhaltenen radialen Verteilungsfunktionen sind in Kapitel 5 dargestellt. Im abschließenden sechsten Kapitel werden die erhaltenen Absorptions- 
zeitprofile, insbesonders im Kurzzeitbereich, diskutiert. Zudem wird erstmals ein kinetisches Modell vorgeschlagen, welches die Dichteabhängigkeit der Isomerisierungsquantenausbeuten und der Bildungsgeschwindigkeiten erklärt. 


\section{Grundlagen}

Nach Photodissoziation eines homonuklearen, zweiatomigen Moleküls unter nicht isolierten Bedingungen sind folgende Prozesse möglich (siehe Abb.2.1 am Beispiel des Iodmoleküls): direkt nach Anregung mit einem Photon dissoziiert das Muttermolekül und die beiden Fragmente entfernen sich voneinander. Anschließend entscheidet sich, ob mindestens eines der Fragmente den ursprünglichen Lösungsmittelkäfig verlassen kann (Käfigausbruch) oder ob beide Fragmente in diesem Käfig gefangen bleiben (Käfigeinfang). Bleiben sie in dem Käfig gefangen, können sie nach Abgabe ihrer kinetischen Energie an das Lösungsmittel rekombinieren (primäre geminale Rekombination). Verlässt mindestens ein Fragment den Käfig, bewegen sich beide Fragmente nach Äquilibrierung der Translationsfreiheitsgrade diffusiv durch das Lösungsmittel. Anschließende Rekombination mit dem ursprünglichen Bindungspartner wird als sekundäre geminale Rekombination bezeichnet, Rekombination mit einem anderen Fragment wird nichtgeminale oder auch homogene Rekombination genannt [2]. Jede Rekombination erzeugt zunächst schwingungsheißes Grundzustandsiod, welches durch Schwingungsenergierelaxation (vibrational energy relaxation, VER) an das Lösungsmittel abkühlt.

Alle drei Rekombinationsprozesse sind durch ihre Zeitskalen eindeutig getrennt. Wegen des eingeschränkten untersuchbaren Zeitintervalls der verwendeten Apparatur (1 ns) beschäftigt sich diese Arbeit ausschließlich mit der geminalen Rekombination.

Abschn.2.1 fasst den derzeitigen Stand der Forschung zum Käfigeffekt des $\mathrm{I}_{2}$-Moleküls zusammen, während die Abschn.2.2 und 2.3 auf den Käfigeffekt des $\mathrm{CH}_{2} \mathrm{I}_{2}$ - und $\mathrm{CH}_{2} \mathrm{BrI-}$ Moleküls eingehen.

\subsection{Der Käfigeffekt des lod-Moleküls}

Bereits in den 1930er Jahren postulierten Franck und Rabinowitsch den photolytischen Käfigeffekt [3, 4]. Danach können die nach Photodissoziation gebildeten Fragmente in einer Lösungsmittelumgebung wieder geminal rekombinieren. Eine solche Reaktion ist demnach von der Konkurrenz zwischen Rekombination und Austritt der Fragmente aus dem Lösungsmittelkäfig bestimmt. In den 1950er Jahren konnte der photolytische Käfigeffekt am Beispiel des Iodmoleküls durch Radikaleinfangexperimente von Noyes und Mitarbeitern beobachtet werden [2, 5, 6, 7, 8, 9]. Die experimentellen Photodissoziationsquantenausbeuten $\Phi_{\text {dis }}$ wurden mit Hilfe eines einfachen Diffusionsmodells diskutiert [2,10]. In späteren Untersuchungen erkannten Luther und Troe den Vorteil des Gebrauchs von überkritischen Fluiden zur Untersuchung des Käfigeffekts und fanden eine Erniedrigung der Photodissoziationsquantenausbeute auf Werte unter eins schon bei weit niedrigeren Dichten als in typischen flüssigen Lösungen [11. In einer weiteren Arbeit wurde bei hohen Drücken ein linearer Zusammenhang zwischen der Quantenausbeute und dem reziproken Quadrat der Lösungsmittelviskosität $\eta^{-2}$ gefun- 


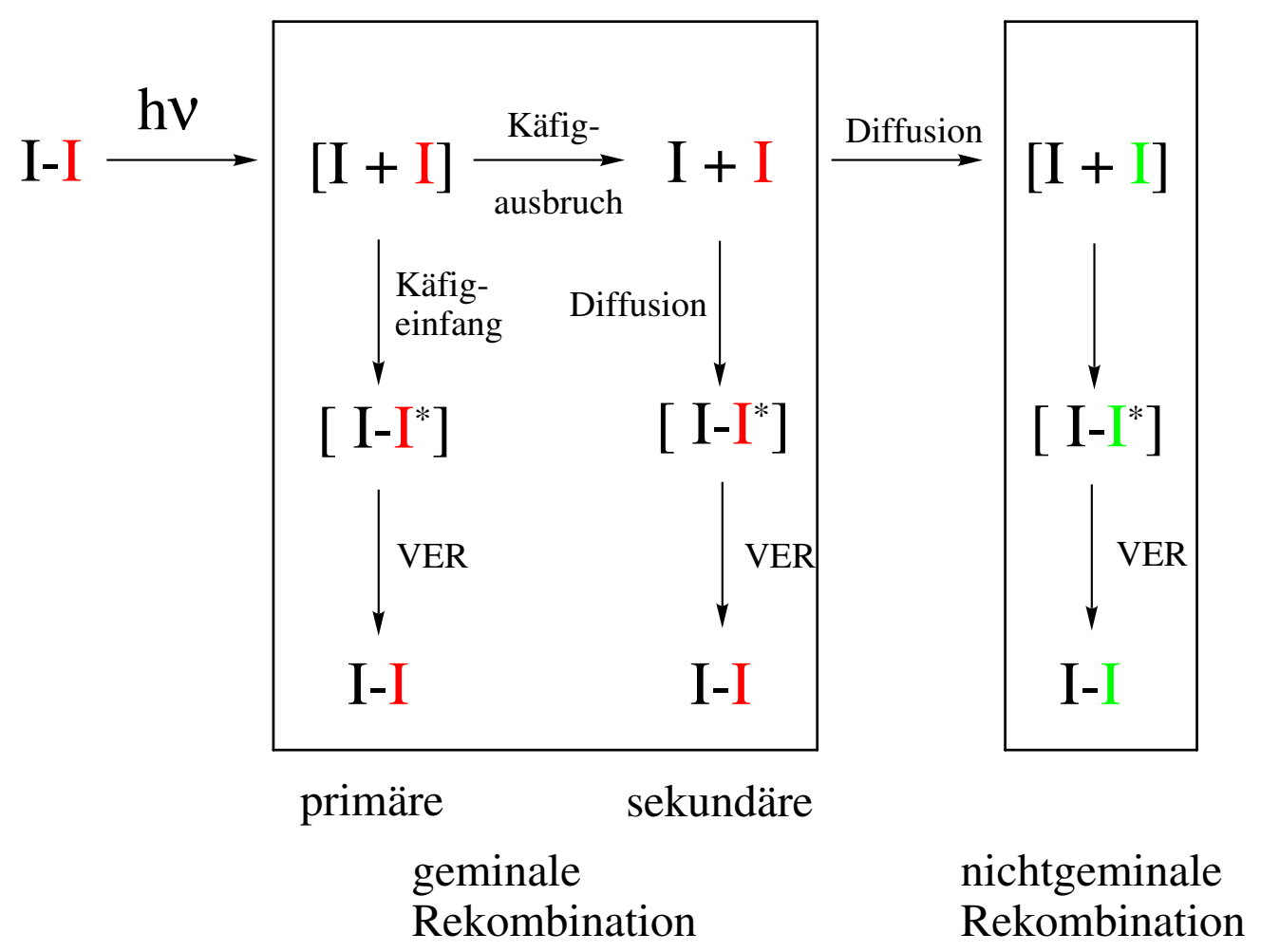

Abbildung 2.1: Reaktionsschema der $\mathrm{I}_{2}$-Photolyse unter nicht isolierten Bedingungen.

den [12. Zewail et al. benutzten ebenfalls überkritische Fluide, um die Dichteabhängigkeit der Ioddissoziation und Rekombination sowie den Lösungsmitteleinfluss auf die Kohärenz der Wellenpaketdynamik zu untersuchen [13, 14].

Weitere Beispiele für den photolytischen Käfigeffekt bei kleinen Molekülen zeigen die in dieser Arbeit untersuchten Dihalogenverbindungen, Iodoform [15, 16, sowie $\mathrm{ClO}_{2}$ [17, 18, $\mathrm{CS}_{2}$ [19, 20], $\mathrm{HOCl}[21,22]$ und ICN [23].

\subsubsection{Das Diffusionsmodell nach Otto, Schroeder und Troe}

Ein einfaches Diffusionsmodell zur Modellierung der Photodissoziationsquantenausbeute $\Phi_{\text {dis }}$, in der das Lösungsmittel als Kontinuum behandelt wird, gibt [24, 25, 26]. Es wird von einer Ortsverteilungsfunktion $n(r)$ ( $r$ : Abstand der Iodatome) der rekombinierenden Iodatome ausgegangen, dessen zeitliches Verhalten sich durch das 2. Ficksche Gesetz beschreiben lässt:

$$
\frac{\partial n(r, t)}{\partial t}=D \frac{\partial^{2} n(r, t)}{\partial r^{2}}
$$

wobei $D$ den Diffusionskoeffizienten der Iodatome im Lösungsmittel darstellt. Nach Photolyse entfernen sich die Iodatome mit einer kinetischen Energie $E_{\text {kin }}=h \nu-E_{0}$, wobei $h \nu$ die Anregungsenergie und $E_{0}$ die Dissoziationsenergie ist. Durch Reibung mit dem Lösungsmittel geben die Iodatome ihre Translationsenergie ab, bis sie bei einem Abstand $r_{0}$ auf thermische Geschwindigkeiten abgebremst werden. Die Randbedingungen obiger Differenti- 
algleichung ergeben sich aus der Annahme, dass die Verteilung zum Zeitnullpunkt $(t=0)$ deltaförmig in $r_{0}$ sei:

$$
n(r, 0)=\left(4 \pi r_{0}^{2}\right)^{-1} \delta\left(r-r_{0}\right)
$$

und bei unendlichem Abstand gegen Null geht:

$$
n(\infty, t)=0
$$

Bei kleineren Entfernungen als dem Kontaktabstand $R$ ist Stoßdesaktivierung möglich. Dies wird durch die Randbedingung

$$
\left.\frac{\partial n(r, t)}{\partial t}\right|_{r=R}=\frac{k_{\mathrm{rec}}^{\mathrm{g}}}{4 \pi R^{2} D N_{\mathrm{A}}} n(R, t)
$$

ausgedrückt, welche in der Literatur auch als "radiation boundary condition“ bekannt ist. $k_{\text {rec }}^{\mathrm{g}}$ ist das Produkt aus Badgaskonzentration $[\mathrm{M}]$ und der Rekombinationsgeschwindigkeitskonstante dritter Ordnung $k_{\text {rec }}$ gemäß d $\left[\mathrm{I}_{2}\right] / \mathrm{d} t=k_{\mathrm{rec}}[\mathrm{M}][\mathrm{I}]^{2}=k_{\mathrm{rec}}^{\mathrm{g}}[\mathrm{I}]^{2}[13$, 24] und $R$ wird aus den Lennard-Jones-Durchmessern $\sigma$ der Iodatome und Badgasmoleküle erhalten: $R \approx[\sigma(\mathrm{I})+\sigma(\mathrm{M})] / \sqrt{2}$.

Die zeitabhängige Dissoziationsquantenausbeute ergibt sich nun aus dem Anteil der Verteilung, der einen größeren Abstand als $R$ hat:

$$
\Phi_{\mathrm{dis}}(t)=\int_{R}^{\infty} 4 \pi r^{2} n(r, t) \mathrm{d} r
$$

Für $t \longrightarrow \infty$ erhält man [27]:

$$
\Phi_{\mathrm{dis}}=1-\frac{R}{r_{0}}\left(\frac{k_{\mathrm{rec}}^{\mathrm{g}}}{k_{\mathrm{rec}}^{\mathrm{g}}+4 \pi R D N_{\mathrm{A}}}\right) .
$$

Der Klammerausdruck beschreibt die Konkurrenz aus der Wahrscheinlichkeit zur Stabilisierung durch Schwingungsenergietransfer gegenüber der diffusiven Separation der Iodatome. Der Faktor $R / r_{0}$ gibt die Wahrscheinlichkeit des Käfigausbruchs gegenüber des Käfigeinfangs an.

Zur Bestimmung von $r_{0}$ wird davon ausgegangen, dass sich die Iodatome nach Anregung mit einer Anfangsgeschwindigkeit von Null auf einem repulsiven Potential bewegen. Das Lösungsmittel dämpft in Form der Stokes'schen Reibungskraft diese Bewegung. Es wird die Bewegungsgleichung für dieses Potential (für das modellhaft ein verkürztes harmonisches angenommen wird) mit obiger Randbedingung gelöst, $r_{0}$ ist der Abstand nach unendlicher Zeit. Es ergibt sich für hinreichend große Viskositäten $r_{0}=R$ und damit für die Dissoziationsquantenausbeute:

$$
\Phi_{\mathrm{dis}}=\frac{4 \pi R D N_{\mathrm{A}}}{k_{\mathrm{rec}}^{\mathrm{g}}+4 \pi R D N_{\mathrm{A}}} .
$$

Bei hohen Dichten kann im Nenner der Diffusionsterm vernachlässigt werden und mit der Annahme $k_{\text {rec }}^{\mathrm{g}} \propto D$ aus diesem Modell und $D \propto \eta^{-1}$ konnte die quadratische Viskositätsabhängigkeit erklärt werden. 


\subsubsection{Dynamische Studien}

Zeitaufgelöste Studien ergeben ein komplexeres Bild der Photodissoziation und Rekombination des Iodmoleküls [28]. Auf Grund der guten Kenntnis der beteiligten elektronischen Zustände (siehe Abb. 2.2] [29] können die Transienten bei verschiedenen Pump- und Probewellenlängen den verschiedenen Prozessen wie Dissoziation, Rekombination und Schwingungsenergietransfer zugeordnet werden. Durch Absorption von Licht im Wellenlängenbereich von 420 bis $720 \mathrm{~nm}$ sind aus dem Grundzustand $\mathrm{X}\left({ }^{1} \Sigma\right)$ die schwach gebundenen Zustände $A\left({ }^{3} \Pi_{1 \mathrm{u}}\right)$ und $\mathrm{A}^{\prime}\left({ }^{3} \Pi_{2 \mathrm{u}}\right)$, der bindende Zustand $\mathrm{B}^{3} \Pi$ sowie der den B-Zustand schneidende repulsive Zustand ${ }^{1} \Pi_{u}$ zugänglich. Kürzere Wellenlängen als $600 \mathrm{~nm}$ bevölkern hauptsächlich den B-Zustand, während Wellenlängen von 660 bis $700 \mathrm{~nm}$ den A/A'-Zustand oberhalb der Dissoziationsschwelle bevölkern. Die Dissoziation erfolgt, je nachdem welcher Zustand anfänglich populiert wird, entweder direkt oder prädissoziativ.

Es lässt sich nun durch transiente Absorption die Population in folgenden Regionen beobachten:

- Der Übergang X $\longrightarrow$ B erlaubt die Observation des Schwingungsenergietransfers im elektronischen Grundzustand.

- Absorption von UV-Wellenlängen in Ionenpaar-Zustände (nicht in Abb.2.2 dargestellt) des Iod-Moleküls erfolgt aus zwei Regionen: aus dem Potentialtopf des metastabilen A/A'-Zustandes und aus den X- und A/A'-Zuständen in der Nähe der Dissoziationsschwelle.

- Die Population im metastabilen A/A'-Zustand kann mit Wellenlängen von 600 bis $800 \mathrm{~nm}$ geprobt werden.

Eine Zusammenfassung der zeitaufgelösten Studien gibt Harris [28]. Das Iodmolekül dissoziiert demnach innerhalb von $2 \mathrm{ps,} \mathrm{unabhängig} \mathrm{davon} \mathrm{in} \mathrm{welchen} \mathrm{Zustand} \mathrm{anfänglich} \mathrm{ange-}$ regt wird. Transiente Absorptionen in der Nähe der Dissoziationsschwelle (350-400 nm) und bei hoch schwingungsangeregten Zuständen des X-Zustandes steigen und fallen innerhalb von 15 ps, was darauf hindeutet, dass die Rekombination innerhalb dieser Zeitspanne abläuft. Die Rekombination in den A/A'-Potentialtopf erfolgt ebenfalls innerhalb 15 ps. Auf Grund der Schnelligkeit dieser Prozesse sprechen beide Ergebnisse gegen einen diffusiven Rekombinationsmechanismus, wie er in Abschn.2.1.1 vorgeschlagen wurde. Die innere Konversion aus dem A/A'-Zustand in den Grundzustand ist stark lösungsmittelabhängig und dauert von $60 \mathrm{ps}$ in Alkanen bis zu $2.7 \mathrm{~ns}$ in $\mathrm{CCl}_{4}$. Die Schwingungsenergierelaxation kann durch das B-X-Absorptionsspektrum verfolgt werden, welches vom Infraroten bis zu $500 \mathrm{~nm}$ blauverschiebt. Diese Blauverschiebung ist ebenfalls lösungsmittelabhängig und erfolgt in organischen Lösungsmitteln in 50-150 ps.

\subsubsection{Kinematischer Käfigeffekt}

Ein anderes Bild des Käfigeffektes ergibt sich aus der Spektroskopie von van-der-Waals Clustern aus Überschallexpansionen. Selbst Anregung oberhalb der Dissoziationsschwelle ermöglicht die Beobachtung eines strukturlosen Fluoreszenzanregungsspektrums, was durch einen einatomigen Käfigeffekt erklärt wurde [30,31]. Danach kann der Komplex, wenn er 


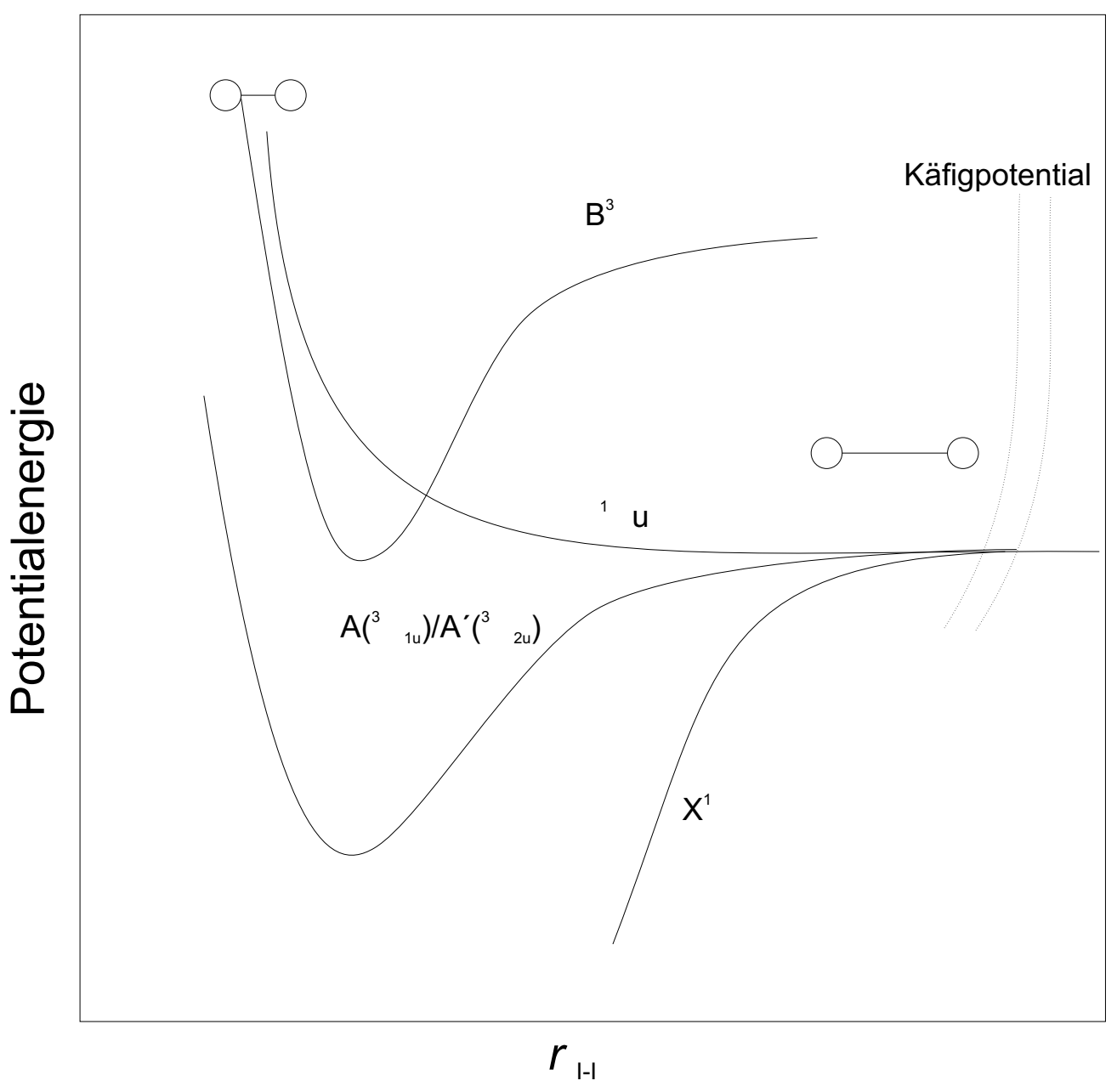

Abbildung 2.2: Potentialflächen des $\mathrm{I}_{2}$.

in einer linearen Geometrie vorliegt [32, nach Anregung seine Überschussenergie durch Kopplung der I - I-Schwingung mit der van-der-Waals Bindung in die relative kinetische Energie des Iodmoleküls und des Edelgasatoms abgeben [33, 34]. Dieser Mechanismus wird als kinematischer Käfigeffekt bezeichnet.

Eine Neubestimmung der Dissoziationsquantenausbeute in überkritischen Lösungsmitteln durch transiente Absorption von Schwarzer et al. [35] ergab, dass die Dissoziationsquantenausbeute mit zunehmender Dichte linear abnimmt. Eine Auftragung der Quantenausbeute verschiedener Lösungsmittel ( $n$-Alkane, $\mathrm{CO}_{2}$ und $\mathrm{Xe}$ ) gegen die reduzierte Dichte ergibt, dass die Quantenausbeute unabhängig von der Natur des Lösungsmittels ist und nur von der reduzierten Dichte abhängt. Dabei ist die reduzierte Dichte ein Maß für die molekulare Packungsdichte und bestimmt, wieviel Raum den dissoziierenden Atomen zur Verfügung steht. Nach dem OST-Modell (siehe Abschn.2.1.1) hingegen korreliert der Käfigeffekt mit dem Diffusionskoeffizienten und dadurch mit der Lösungsmittelviskosität. Die Korrelation der Photodissoziationsquantenausbeute mit der Packungsdichte unterstützt demnach das Bild eines rein kinematischen Käfigausbruchs. 
Quantenausbeuten für die Ioddissoziation wurden durch ebenfalls in überkritischer Lösung durchgeführte Transient Grating Experimente von Ooe et al. [36] erhalten. Die gemessenen Quantenausbeuten dieser Arbeit liegen in der gleichen Größenordnung wie die von Schwarzer et al. Desweiteren lassen sich die in verschiedenen Lösungsmitteln erhaltenen Quantenausbeuten ebenfalls durch die reduzierte Dichte miteinander vergleichen, jedoch ist der funktionelle Zusammenhang von Quantenausbeute und Dichte in der Arbeit von Ooe et al. nicht linear.

Klassische MD-Simulationen in $\mathrm{CCl}_{4}$ [37] und in komprimierten Edelgasen [38, 39] zeigen, dass die Rekombination vollständig innerhalb der ersten Lösungsmittelschale verläuft und die sekundäre geminale Rekombination vernachlässigbar ist.

\subsection{Der Käfigeffekt des Diiodmethan-Moleküls}

Der Käfigeffekt des $\mathrm{I}_{2}$ gilt als gut verstanden und so erscheint es sinnvoll, die Untersuchungen auf kompliziertere Systeme auszuweiten. Als Modellsystem hat sich das polyatomare Molekül $\mathrm{CH}_{2} \mathrm{I}_{2}$ herausgestellt. Hauptunterschied zum $\mathrm{I}_{2}$ ist, dass eines der Photofragmente (nämlich das $\mathrm{CH}_{2} \mathrm{I}$-Radikal) innere Freiheitsgrade besitzt. Zum anderen wird die Rekombination der Fragmente in das Muttermolekül nicht beobachtet, sondern es bildet sich eine Iod-Iod-Bindung aus und es findet eine Isomerisierung zum $\mathrm{CH}_{2} \mathrm{I}-\mathrm{I}$ statt.

\subsubsection{Spektroskopie und Dissoziationsdynamik}

Die Dissoziation des Diiodmethans in der Gasphase ist gut untersucht und diese Ergebnisse werden zur Interpretation der in dieser Arbeit erhaltenen Messungen herangezogen. Diiodmethan liegt in der Gasphase in $\mathrm{C}_{2 \mathrm{v}}$-Symmetrie vor. Das stationäre Gasphasen Absorptionsspektrum des Diiodmethans (siehe Abb.2.3) lässt sich mit vier gaußförmigen Banden der Zentralwellenlängen 312, 284, 249 und $209 \mathrm{~nm}$ anpassen [40]. Durch ein einfaches ExcitonModell [41] lassen sich diese Banden fünf elektronischen Zuständen der Symmetrie $1 \mathrm{~B}_{1}, 2 \mathrm{~B}_{1}$, $\mathrm{B}_{2}, 1 \mathrm{~A}_{1}$ und $2 \mathrm{~A}_{1}$ zuordnen, wobei der $\mathrm{B}_{2^{-}}$und $1 \mathrm{~A}_{1}$-Zustand in der $249 \mathrm{~nm}$-Bande nicht einzeln aufgelöst werden können [42]. Das Übergangsdipolmoment für die Anregung in beiden niederenergetischen Zustände der $S_{1}$-Absorptionsbande $\mathrm{B}_{1} \longleftarrow$ A, bzw. $2 \mathrm{~B}_{1} \longleftarrow$ A liegt parallel zur I-I-Verbindungslinie [43]. Eine Zusammenfassung der elektronischen Zustände gibt Abb.2.4.

Nach UV-Anregung sind folgende Reaktionskanäle möglich [41,44]:

$$
\begin{array}{ll}
\mathrm{CH}_{2} \mathrm{I}_{2} \stackrel{h \nu}{\longrightarrow} \mathrm{CH}_{2} \mathrm{I}+\mathrm{I}\left({ }^{2} \mathrm{P}_{3 / 2}\right) & \lambda<557 \mathrm{~nm}, \\
\mathrm{CH}_{2} \mathrm{I}_{2} \stackrel{h \nu}{\longrightarrow} \mathrm{CH}_{2} \mathrm{I}+\mathrm{I}^{*}\left({ }^{2} \mathrm{P}_{1 / 2}\right) & \lambda<392 \mathrm{~nm}, \\
\mathrm{CH}_{2} \mathrm{I}_{2} \stackrel{h \nu}{\longrightarrow} \mathrm{CH}_{2}+\mathrm{I}_{2} & \lambda<333 \mathrm{~nm} .
\end{array}
$$

Die Reaktion R10 ist symmetrieverboten [45], wurde experimentell jedoch durch Multiphotonenabsorption realisiert [46, 47, 48]. Diese zeitaufgelösten Studien zeigten, dass die Reaktion konzertiert in < 100 fs abläuft.

Wellenlängenabhängige Quantenausbeuten für die Reaktionen $\mathrm{R} 8$ und $\mathrm{R} 9$ wurden von Koffend und Leone [49] über die Emission des Übergangs $\mathrm{I}^{*}\left({ }^{2} \mathrm{P}_{1 / 2}\right) \longrightarrow \mathrm{I}\left({ }^{2} \mathrm{P}_{3 / 2}\right)$ sowie von 


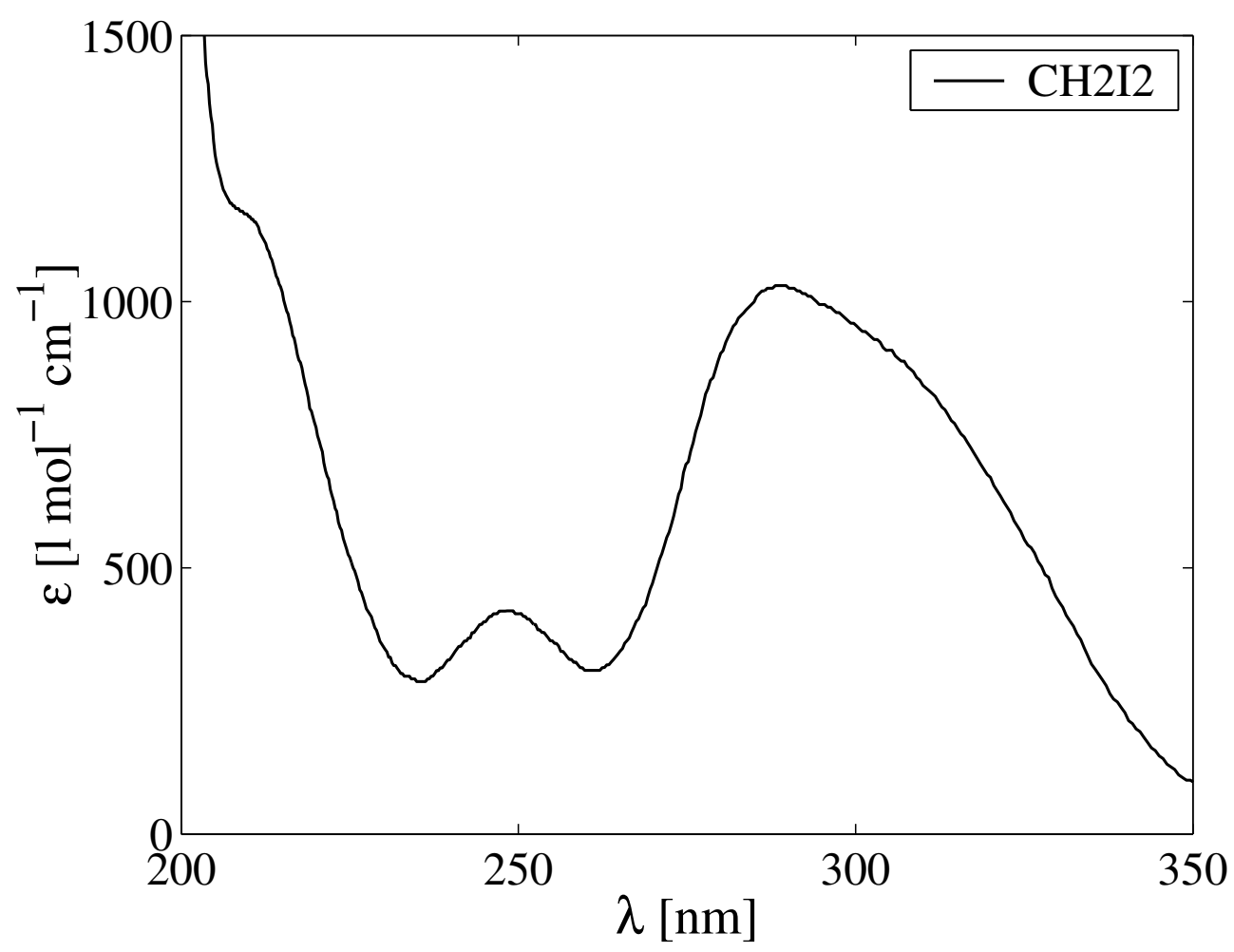

Abbildung 2.3: Gasphasen Absorptionsspektrum des $\mathrm{CH}_{2} \mathrm{I}_{2}$ [42].

Hunter und Kristjansson [50] mit photoakkustischen Methoden gemessen. Einen Vergleich beider Studien zeigt Abb.2.5. Aus diesen Messungen wurde geschlossen, dass die Reaktion aus dem $1 \mathrm{~B}_{1}$-Zustand zur Bildung von Grundzustands-Iodatomen führt, während die Reaktion aus dem $2 \mathrm{~B}_{1}$-Zustand $\mathrm{I}^{*}\left({ }^{2} \mathrm{P}_{1 / 2}\right)$ bildet. Eine Kurvenkreuzung mit energetisch höher liegenden elektronischen Zuständen (siehe Abb.2.4) führt zur Verminderung der Quantenausbeute $\Phi\left(\mathrm{I}^{*}\right)$ [49].

$I^{*}\left({ }^{2} \mathrm{P}_{1 / 2}\right)$ kann durch Stöße mit $\mathrm{CH}_{2} \mathrm{I}_{2}$ effektiv desaktiviert werden. Die Geschwindigkeitskonstante $k_{1}$ der Reaktion $\mathrm{CH}_{2} \mathrm{I}_{2}+\mathrm{I}^{*}\left({ }^{2} \mathrm{P}_{1 / 2}\right) \longrightarrow \mathrm{CH}_{2} \mathrm{I}_{2}+\mathrm{I}\left({ }^{2} \mathrm{P}_{3 / 2}\right)$ konnte zu $(3.4 \pm 0.4) \cdot 10^{-13} \mathrm{~cm}^{3}$ molecule ${ }^{-1} \mathrm{~s}^{-1}\left[50\right.$ ] bzw. $(3.6 \pm 0.3) \cdot 10^{-13} \mathrm{~cm}^{3}$ molecule ${ }^{-1} \mathrm{~s}^{-1}$ [40] bestimmt werden. Die angeregten Iodatome können ebenfalls durch Stöße mit $\mathrm{I}_{2}\left(k_{1}=\right.$ $(3.1 \pm 0.5) \cdot 10^{-11} \mathrm{~cm}^{3}$ molecule $\left.\mathrm{s}^{-1}\right)$ desaktiviert werden, die Lebensdauer für den strahlenden Übergang ist mit $\sim 0.1 \mathrm{~s}$ hingegen zu vernachlässigen [51.

Abb.2.6 zeigt das Gasphasen Absorptionsspektrum des $\mathrm{CH}_{2}$ I-Radikals 52 im Vergleich mit dem $\mathrm{CH}_{2} \mathrm{Br}$-Radikal [53]. Das Spektrum der Iodverbindung weist zwei intensive Banden bei $\sim 280$ und $\sim 340 \mathrm{~nm}$ auf, wobei die langwellige mit der Bande des Isomers überlappt (siehe nächster Abschnitt).

Die Überschussenergie $E_{\text {exc }}$, die den Fragmenten als kinetische Energie $E_{\text {kin }}$, Rotations$E_{\mathrm{r}}$ und Schwingungsenergie $E_{\mathrm{v}}$ zur Verfügung steht, lässt sich durch $E_{\text {exc }}=h \nu-\Delta_{\mathrm{r}} H$ abschätzen, wobei sich die Reaktionsenthalpie $\Delta_{\mathrm{r}} H$ aus den Standardbildungsenthalpien der beteiligten Reaktionspartner [54,55] ergibt. Flugzeitmassenspektroskopische Messungen bei $266 \mathrm{~nm}$ Anregung zeigen, dass 80-90\% dieser Überschussenergie in die innere Ener- 


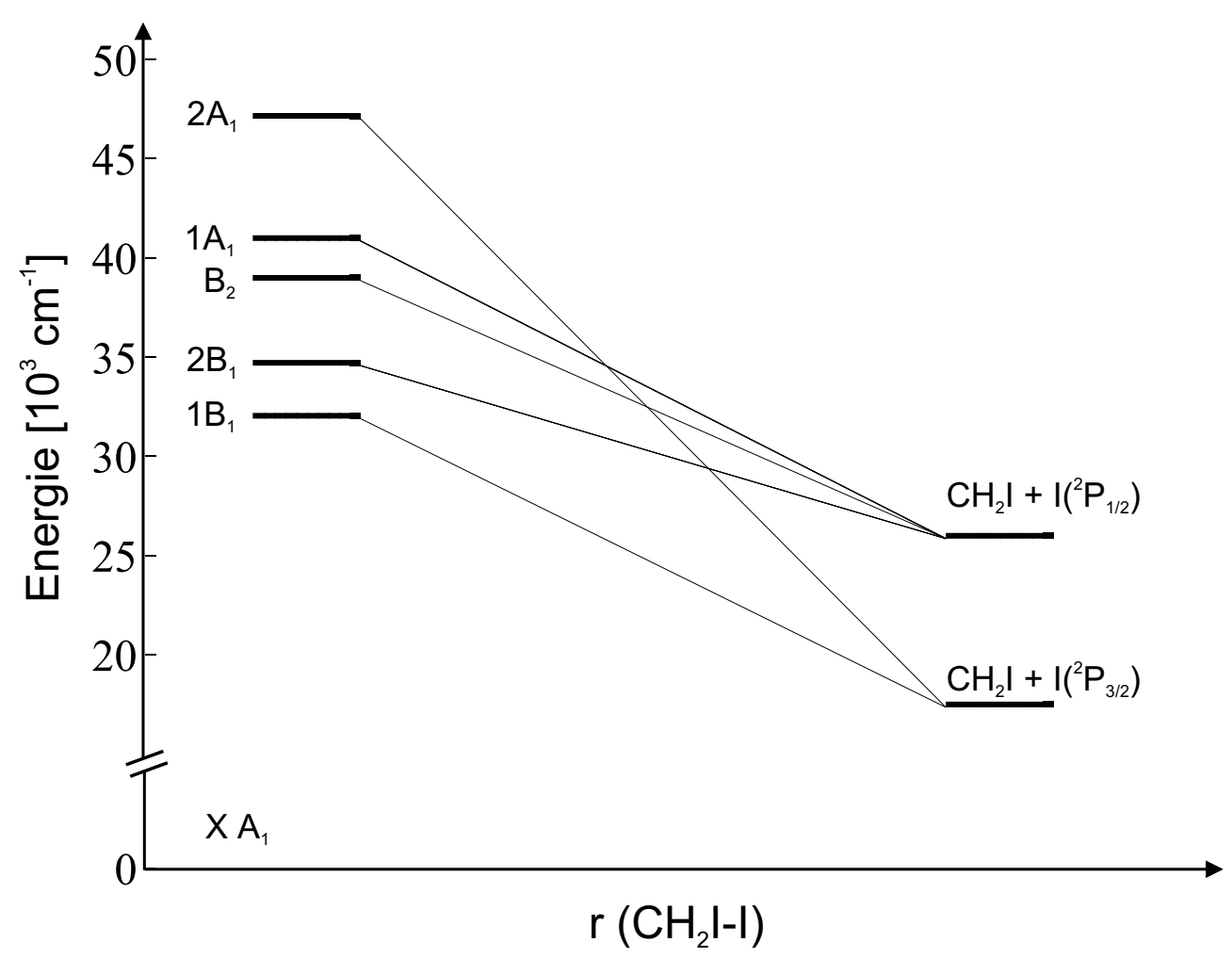

Abbildung 2.4: Energiediagramm der elektronischen Zustände des $\mathrm{CH}_{2} \mathrm{I}_{2}$ mit Darstellung der Dissoziationskanäle.

gie $E_{\text {in }}=E_{\mathrm{r}}+E_{\mathrm{v}}$ des Radikals fließt [43]. Neuere Untersuchungen zeigen, dass die prozentuale Verteilung der Überschussenergie in innere und Translationsfreiheitsgrade nur geringfügig von der Anregungsenergie abhängt [42. Weiterhin wurde abgeschätzt, dass der Anteil der Rotationsenergie $E_{\mathrm{r}}$ an der inneren Energie $E_{\text {in }}$ etwa $40 \%$ beträgt [43].

Informationen über die Dynamik und die Zeitskala der Dissoziation in der Gasphase wurden aus LIF-Spektren und der Winkelverteilung der Photofragmente gewonnen. Im LIFSpektrum lassen sich folgende Banden beobachten: die symmetrische $\mathrm{CI}_{2}$-Streckschwingung $\left(\nu_{3}\right)$ mit Progression, die Fundamentale der ICI-Knickschwingung $\left(\nu_{4}\right)$, eine Kombinationsbande aus $\nu_{3}$ und der asymmetrischen $\mathrm{CI}_{2}$-Streckschwingung $\left(\nu_{9}\right)$ sowie die $2 \nu_{9}$-Bande [44]. Auf Grund der guten Sichtbarkeit der symmetrischen Streckschwingung wurde darauf geschlossen, dass das Wellenpaket hauptsächlich auf der Potentialfläche der symmetrischen Streckschwingung gebildet wird, jedoch wird auch ein Teil des Wellenpakets auf der Potentialfläche der asymmetrischen Streckschwingung gebildet. Diese Potentialfläche muss stark repulsiven Charakter haben, weil nur ein Oberton der Schwingung $\nu_{9}$ sichtbar ist. Dass die Dissoziation schnell $(<100 \mathrm{fs}$ ) verläuft, konnte ebenfalls aus der Winkelverteilung der Photofragmente geschlossen werden [41. Nach diesen Untersuchungen ist die Dissoziation schnell gegenüber einer Rotationsperiode, was wiederum auf einen repulsiven Charakter der Potentialfläche hindeutet. 


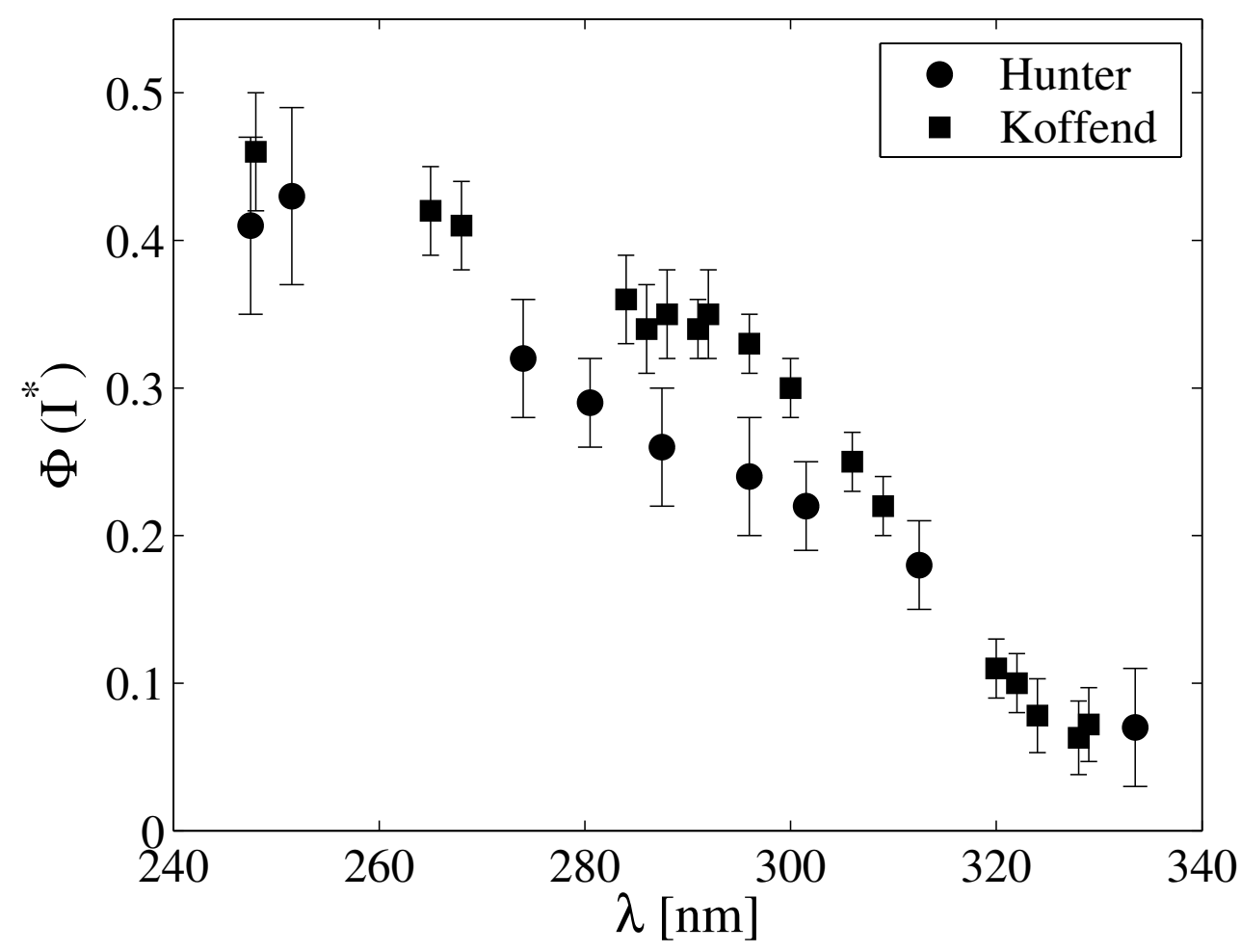

Abbildung 2.5: Wellenlängenabhängige Quantenausbeuten $\Phi\left(\mathrm{I}^{*}\right)$ nach Photodissoziation des $\mathrm{CH}_{2} \mathrm{I}_{2}$ [49, 50].

\subsection{2 $\mathrm{CH}_{2} \mathrm{I}_{2}$ in der kondensierten Phase}

Die Dissoziation in organischen Lösungsmitteln wurde durch zeitaufgelöste Resonanz-Raman Messungen untersucht. Diese Untersuchungen ergaben, dass die $C_{2 \mathrm{v}}$-Symmetrie des $\mathrm{CH}_{2} \mathrm{I}_{2}$ in Lösung gebrochen wird. Während in der Gasphase zu Beginn der Dissoziationsdynamik nicht zwischen den beiden C-I-Bindungen unterschieden wird, wird in Lösung die Streckung einer der beiden C-I-Bindungen durch Solvatation gehindert [56].

Nach UV-Anregung von Diiodmethan in einer Argon oder Stickstoff Matrix bei $12 \mathrm{~K}$ [57, 58, wird eine neue Verbindung gebildet, welche ein charakteristisches UV/VIS- und IR-Spektrum aufweist. Das UV/VIS-Spektrum (siehe Abb.2.7) zeigt eine Bande größerer Intensität bei $\simeq 370 \mathrm{~nm}$ und eine weniger intensive bei $\simeq 545 \mathrm{~nm}$. Durch Anregung in die beiden Absorptionsbanden kann das Muttermolekül fast quantitativ zurückgebildet werden. Auf Grund der Reversibilität der Reaktion wurde geschlossen, dass es sich bei dieser neuen Verbindung um ein Isomer handelt, bei dem das anfänglich dissoziierte Iodatom an das verbliebene Iodatom gebunden ist. Durch Vergleich der IR-Spektren mit durch $a b$ initio-Methoden berechneten Frequenzen wurde diese Struktur bestätigt. Weiterführende $a b$ initio-Rechnungen [59, 60] bestätigen den bindenden Charakter der Halogen-HalogenBindung. Nach diesen neueren Rechnungen beträgt die Barriere für die Rückisomerisierung in das Muttermolekül $+134.6 \mathrm{~kJ} / \mathrm{mol}$ und die Enthalpieänderung für die homolytische Disso- 


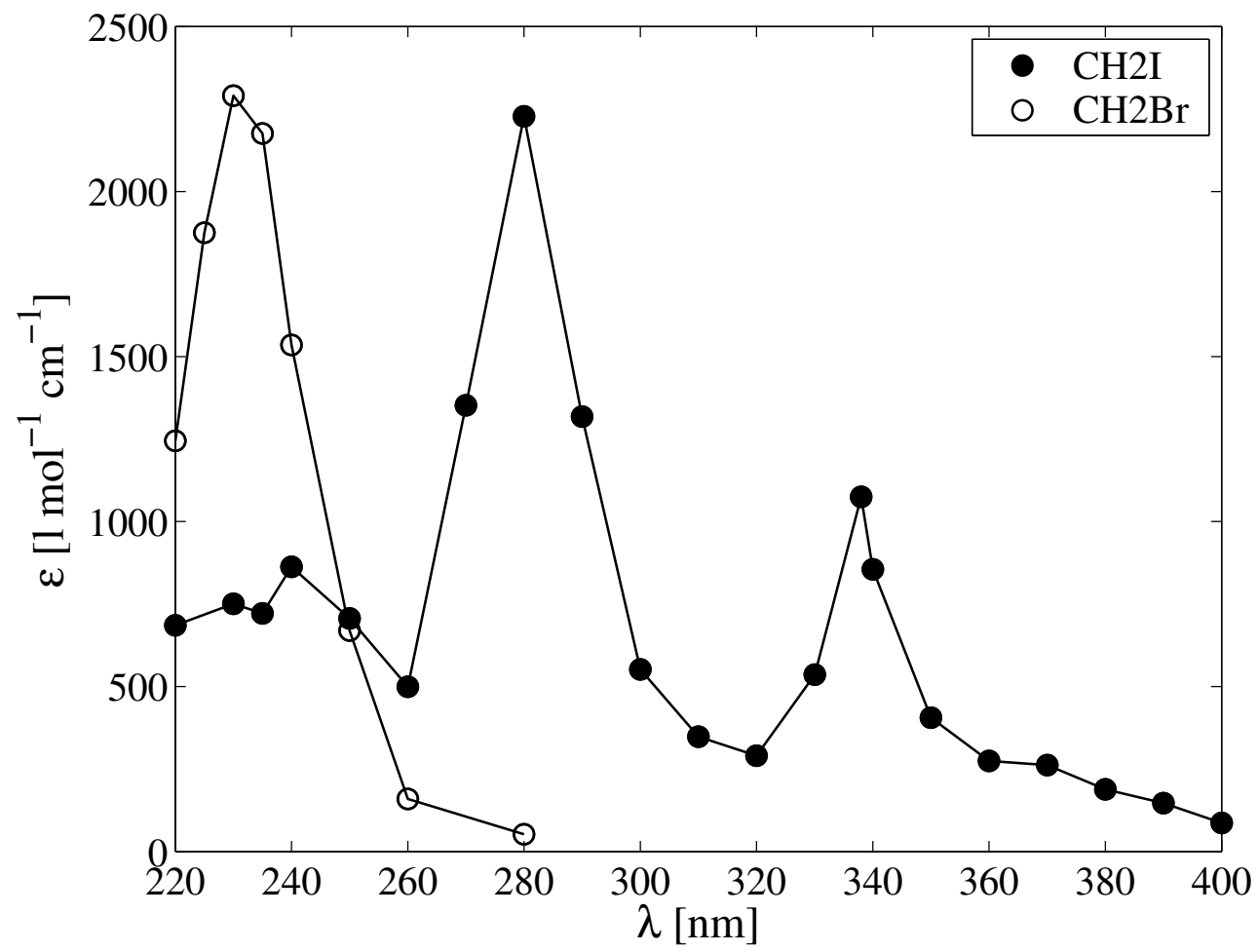

Abbildung 2.6: Gasphasen Absorptionsspektren des $\mathrm{CH}_{2} \mathrm{I}$ [52] und $\mathrm{CH}_{2} \mathrm{Br}$ [53].

ziation $\left(\mathrm{CH}_{2} \mathrm{I}-\mathrm{I} \longrightarrow \mathrm{CH}_{2} \mathrm{I}+\mathrm{I}\right) \Delta_{\text {dis }} H=+19.4 \mathrm{~kJ} / \mathrm{mol}$ [59]. Weiterhin ergab sich aus diesen theoretischen Untersuchungen eine $C_{\mathrm{s}}$-Symmetrie für das Isomer.

Zeitabhängige Absorptionsspektren nach Photolyse von $\mathrm{CH}_{2} \mathrm{I}_{2}$ mit Femtosekunden Auflösung in Acetonitril [61] zeigen eine ähnliche Bandenstruktur wie die Matrixspektren, woraus geschlossen wurde, dass das Isomer auch in Lösung gebildet wird. Die Absorptionszeitprofile zeigen im Wesentlichen drei Komponenten: einen schnellen Anstieg innerhalb der Zeitauflösung des Experiments, einen schnellen Abfall ( $\simeq 350 \mathrm{fs})$ und einen langsamen Anstieg in der Größenordnung von 10 ps. Der schnelle instantane Anstieg wird der transienten Absorption von der repulsiven $\mathrm{S}_{1}$-Potentialfläche in höhergelegene elektronische Niveaus zugeordnet. Der darauf folgende schnelle Abfall wird mit der Bewegung des auf dieser repulsiven Potentialfläche gebildeten Wellenpakets aus der Franck-Condon-Region erklärt. Der abschließende langsame Anstieg wird der Bildung des Isomers zugeschrieben. Weil die Absorption nach dem schnellen Abfall auf ein von Null verschiedenes Minimum abfällt und das Spektrum nach $\sim 1$ ps eine ähnliche Form wie das Langzeitspektrum aufweist, wurde angenommen, dass die Isomerbildung auf einer schnellen und einer langsamen Zeitskala abläuft [62]. Für die schnelle Komponente wurde eine Zeitkonstante von 1 ps abgeschätzt während aus den frequenzintegrierten Spektren eine Bildungsgeschwindigkeit von 5.5 ps erhalten wurde [63]. Die zeitaufgelösten Spektren zeigen zusätzlich zur Populationskinetik eine spektrale Evolution die sich in einer zeitlichen Verschmälerung äußert. Zugeordnet wurde dieses Verhalten der Schwingungsrelaxation des anfänglich schwingungsheiß gebildeten Isomers. Der vorgeschlagene Mechanismus beinhaltet Dissoziation eines Iodatoms, anschließenden Stoß der Frag- 


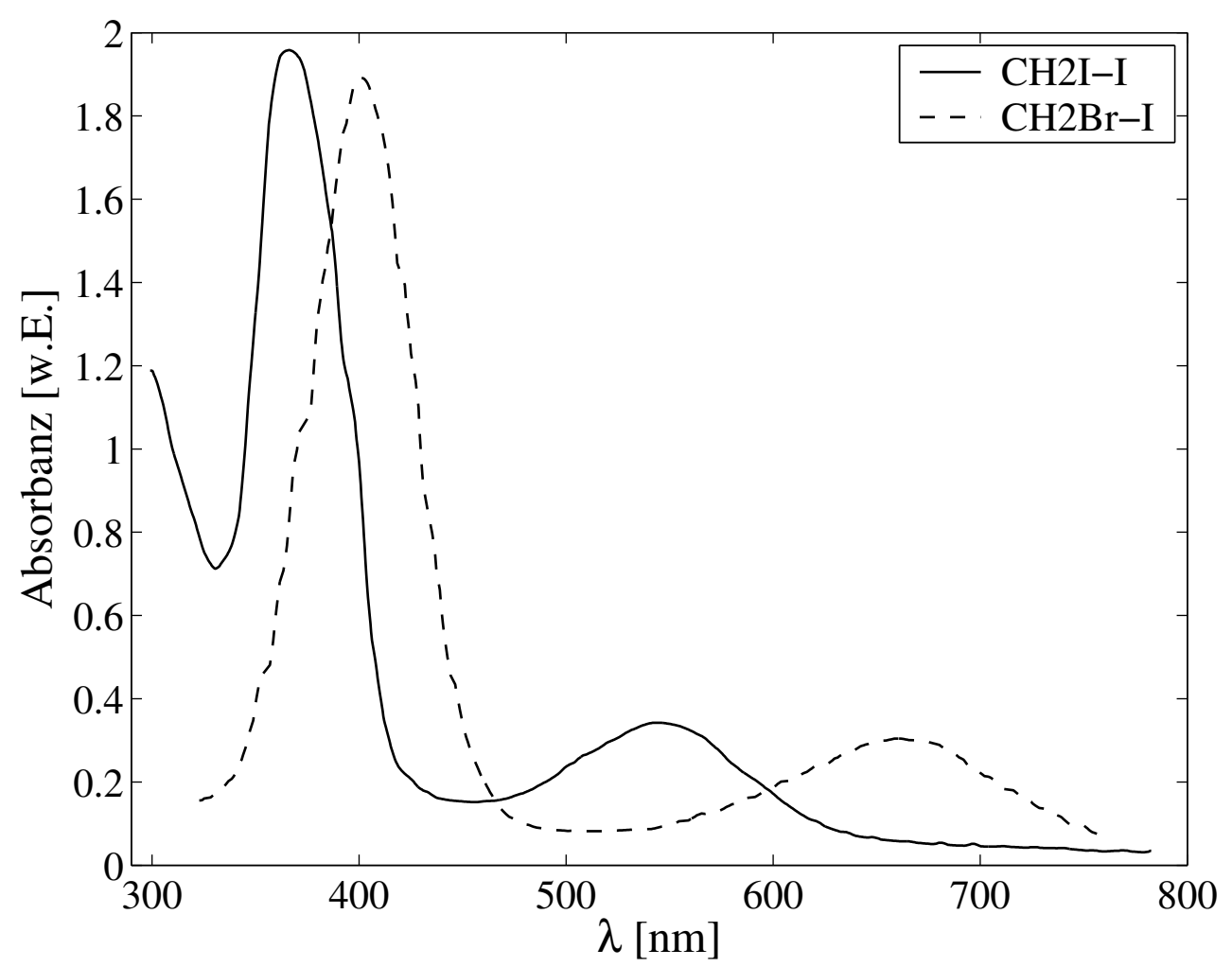

Abbildung 2.7: Absorptionsspektren von $\mathrm{CH}_{2} \mathrm{I}-\mathrm{I}$ und $\mathrm{CH}_{2} \mathrm{Br}-\mathrm{I}$ in einer Stickstoff-Matrix 58.

mente mit dem Lösungsmittelkäfig und Rekombination. Ausgehend von den Extinktionskoeffizienten der stationären Matrix-Absorptionsspektren $(\varepsilon(370 \mathrm{~nm}) \sim 10000 \mathrm{l} /(\mathrm{mol} \cdot \mathrm{cm})$ und $\varepsilon(545 \mathrm{~nm}) \sim 1300 \mathrm{l} /(\mathrm{mol} \cdot \mathrm{cm})$ [57]) wurden die Quantenausbeuten für die Bildung des Isomers in verschiedenen organischen Lösungsmitteln abgeschätzt [63. Die Quantenausbeute ist lösungsmittelabhängig und beträgt zwischen 70 und $90 \%$. Ebenso wurde die bathochrome Verschiebung des Isomerspektrums vom unpolaren Lösungsmittel $n$-Hexan bis zum polaren Lösungsmittel Acetonitril mit $8 \mathrm{~nm}$ in der UV-Bande sowie $4 \mathrm{~nm}$ in der VIS-Bande bestimmt.

Ebenfalls bewiesen wurde die Bildung des Isomers in Lösung durch transiente ResonanzRaman Spektroskopie [64, 65]. Die hier experimentell zugänglichen Schwingungsfrequenzen wurden mit durch ab initio-Methoden gerechneten Frequenzen des Isomers, des $\mathrm{CH}_{2} \mathrm{I}$ Radikals und des $\mathrm{CH}_{2} \mathrm{I}_{2}{ }^{+}$-Kations verglichen, wobei als Zuordnung nur das Isomer in Frage kam. Die Banden enstanden in Acetonitril ebenfalls nach 5 ps, um anschließend an Intensität zu gewinnen und zu höheren Frequenzen zu verschieben. Dieses Verhalten wurde ebenfalls durch Schwingungsrelaxation des heißen Isomers erklärt.

Untersuchungen mit Nanosekunden Zeitauflösung zeigen, dass das Isomer bis zu mehreren Mikrosekunden stabil ist und mit einer Mischung aus Reaktionen erster und zweiter Ordnung zerfällt 63 . 


\subsection{Der Käfigeffekt des Bromiodmethan-Moleküls}

Im Gegensatz zum Diiodmethan besitzt das Bromiodmethan zwei verschiedene Chromophore und somit ergibt sich die Frage nach der Selektivität der Photodissoziation. Während unter thermischen Bedingungen die schwächere C-I-Bindung gebrochen wird, hängt die Selektivität nach Photoanregung von den anfänglich populierten elektronischen Zuständen und möglicherweise auftretenden Kreuzungen ab.

\subsubsection{Spektroskopie und Dissoziationsdynamik}

Das Gasphasen-Absorptionsspektrum von $\mathrm{CH}_{2} \mathrm{BrI}$ weist drei Banden mit den Maxima bei $\sim 270, \sim 215$ und $\sim 190 \mathrm{~nm}$ auf (siehe Abb. 2.8) [66, 67]. Die beiden langwelligen Banden bei 270 und $215 \mathrm{~nm}$ wurden durch Vergleich mit den Absorptionsspektren der einfach halogenierten Verbindungen $\mathrm{CH}_{3} \mathrm{I}$ und $\mathrm{CH}_{3} \mathrm{Br} \operatorname{dem} \sigma^{*}(\mathrm{C}-\mathrm{I}) \longleftarrow n(\mathrm{I})-$, bzw. dem $\sigma^{*}(\mathrm{C}-\mathrm{Br}) \longleftarrow n(\mathrm{Br})-$ Übergang zugeordnet. Beide Banden ergeben sich als Superposition des Übergangs in drei verschiedene elektronisch angeregte Zustände: ${ }^{3} \mathrm{Q}_{1},{ }^{3} \mathrm{Q}_{0}$ und ${ }^{1} \mathrm{Q}_{1}$ [67] (in energetisch steigender Reihenfolge). Die kurzwellige Bande wurde Rydberg-Übergängen des Iod-Atoms zugeordnet [66, 68, 69]. Das Absorptionsspektrum des $\mathrm{CH}_{2}$ Br-Radikals [53] findet sich in Abb.22.6.

Folgende Reaktionen wurden nach Absorption eines Photons im Molekularstrahl beobachtet [68,69]:

$$
\begin{aligned}
& \mathrm{CH}_{2} \mathrm{BrI} \stackrel{h \nu}{\longrightarrow} \mathrm{CH}_{2} \mathrm{Br}+\mathrm{I}\left({ }^{2} \mathrm{P}_{3 / 2}\right) \\
& \mathrm{CH}_{2} \mathrm{BrI} \stackrel{h \nu}{\longrightarrow} \mathrm{CH}_{2} \mathrm{Br}+\mathrm{I}^{*}\left({ }^{2} \mathrm{P}_{1 / 2}\right) \\
& \mathrm{CH}_{2} \mathrm{BrI} \stackrel{h \nu}{\longrightarrow} \mathrm{CH}_{2} \mathrm{I}+\mathrm{Br}\left({ }^{2} \mathrm{P}_{3 / 2}\right) \\
& \mathrm{CH}_{2} \mathrm{BrI} \stackrel{h \nu}{\longrightarrow} \mathrm{CH}_{2} \mathrm{I}+\mathrm{Br}^{*}\left({ }^{2} \mathrm{P}_{1 / 2}\right)
\end{aligned}
$$

Die Quantenausbeuten für die jeweiligen Reaktionskanäle sind im starken Maße eine Funktion der Anregungswellenlänge. Es wird davon ausgegangen, dass Anregung in die Bande $\operatorname{des} \sigma^{*}(\mathrm{C}-\mathrm{I}) \longleftarrow n(\mathrm{I})$-Übergangs zur selektiven Dissoziation eines Iodatoms, Anregung in die Bande des $\sigma^{*}(\mathrm{C}-\mathrm{Br}) \longleftarrow n(\mathrm{Br})$-Übergangs zur Dissoziation eines Bromatoms führt; eine Kurvenkreuzung zwischen beiden Kanälen kann dementsprechend ausgeschlossen werden [66, 69]. Im Wellenlängenbereich um $255 \mathrm{~nm}$ überlappen beide Banden.

Bei $248 \mathrm{~nm}$ Anregung ergab sich die Dissoziation eines I-Atoms als Hauptreaktion und hierbei ein Verhältnis von R11 zu R12 von $1.0: 0.75$ [69].

\subsection{2 $\mathrm{CH}_{2} \mathrm{BrI}$ in der kondensierten Phase}

In Analogie zu den Matrixuntersuchungen des $\mathrm{CH}_{2} \mathrm{I}_{2}$ wurde das $\mathrm{CH}_{2} \mathrm{Br}$-I-Isomer ebenfalls zuerst in Tieftemperatur Matrizen durch sein charakteristisches Absorptionsspektrum (siehe Abb. 2.7) nachgewiesen [57, 58]. Transiente Resonanz-Raman Messungen in Cyclohexan in Verbindung mit DFT-Rechnungen zeigten, dass auch Isomere von $\mathrm{CH}_{2} \mathrm{BrI}$ in Lösung gebildet werden [70,71]. Diese Experimente, die sowohl mit Nano- als auch mit Pikosekundenzeitauflösung durchgeführt wurden, ergaben, dass sich bei geeigneter Anregungswellenlänge nach 


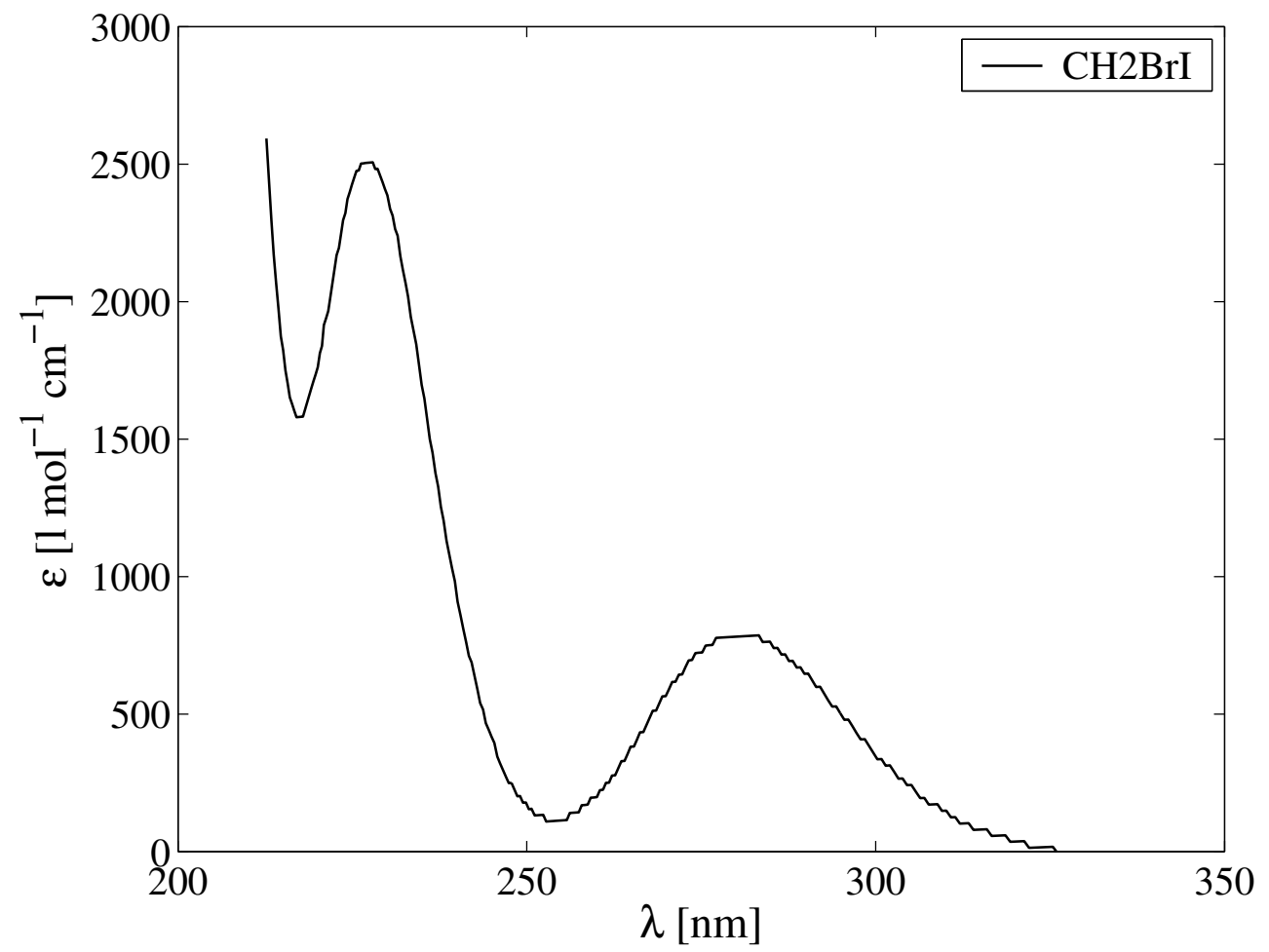

Abbildung 2.8: Gasphasen Absorptionsspektrum des $\mathrm{CH}_{2} \mathrm{BrI}$ [67].

Abspaltung des Iodatoms innerhalb weniger Pikosekunden erst das $\mathrm{CH}_{2} \mathrm{Br}$-I-Isomer bildet, welches sich anschließend im Zeitbereich von 2-3 ns in das thermodynamisch stabilere $\mathrm{CH}_{2} \mathrm{I}$-Br-Isomer umlagert. Die DFT-Rechnungen ergaben eine relative Stabilisierung des $\mathrm{CH}_{2} \mathrm{I}-\mathrm{Br}$-Isomers von $20 \mathrm{~kJ} / \mathrm{mol}$ gegenüber der $\mathrm{CH}_{2} \mathrm{Br}-\mathrm{I}$-Verbindung. Desweiteren wurde eine beträchtliche Kopplung zwischen den C-I- und C-Br-Chromophoren im Muttermolekül gefunden [67, 72].

Ebenfalls wurde die Bildung des $\mathrm{CH}_{2} \mathrm{Br}$-I-Isomers in Acetonitril in Analogie zur Bildung des $\mathrm{CH}_{2} \mathrm{I}-\mathrm{I}$ durch zeitaufgelöste Absorptionsmessungen bei $266 \mathrm{~nm}$ Anregung beobachtet 73. Die Umlagerung in das stabilere Isomer konnte wegen des kürzeren untersuchten Zeitbereichs dieser Arbeit nicht beobachtet werden. Die Spektren zeigen eine Bildung innerhalb von 7.5 ps und ebenfalls eine für den Schwingungsenergietransfer charakteristische Verschmälerung. Desweiteren zeigten die Absorptionszeitprofile die gleichen drei Charakteristika wie die bei der Isomerisierung des Diiodmethans. Die Quantenausbeute für die Isomerbildung in Acetonitril wurde zu $0.26 \pm 0.05$ abgeschätzt.

\subsection{Solvatation in überkritischen Fluiden}

Ein Fluid ist dann überkritisch, wenn seine Temperatur und sein Druck über dem kritischen Punkt liegen [74]. Bei diesen Bedingungen verbindet das Fluid Eigenschaften von Gasen und Flüssigkeiten: Die Dichte ist hoch genug, um eine gute Löslichkeit zu gewährleisten, hingegen 


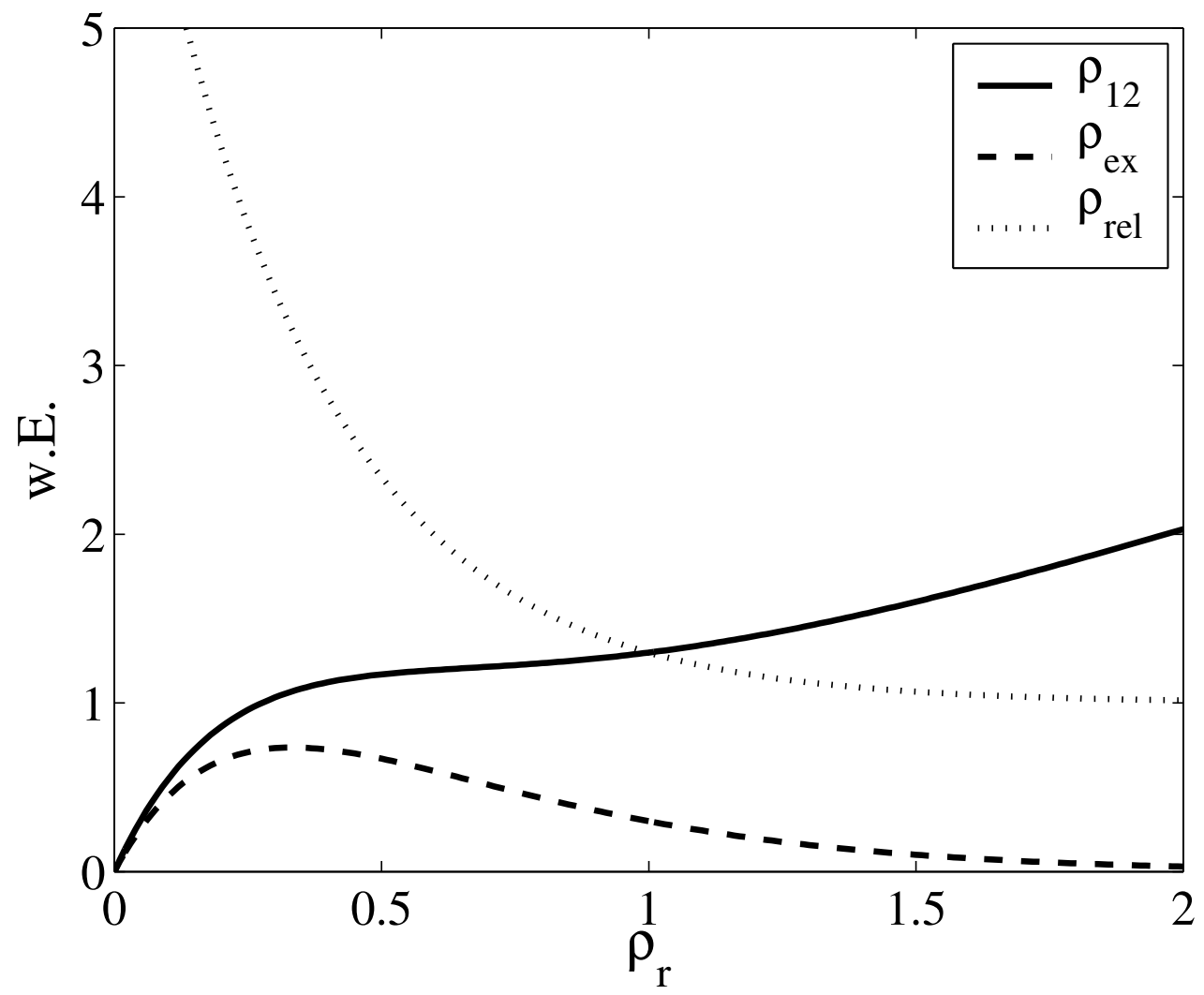

Abbildung 2.9: Vergleich der lokalen Dichte $\rho_{12}$, der lokalen Dichtevergrößerung $\rho_{\text {ex }}$ und der relativen lokalen Dichte $\rho_{\text {rel }}$.

sind die Diffusivitäten größer als in Flüssigkeiten, was den Massentransport und somit chemische Reaktionen erleichtert. Zudem sind überkritische Fluide, insbesondere in der Nähe des kritischen Punkts, in starkem Maße inhomogen, d. h. sie weisen zeitlich fluktuierende Bereiche höherer und niedrigerer Dichte auf. Aus diesen Dichtefluktuationen resultiert die hohe Kompressibilität überkritischer Fluide, die zur Folge hat, dass durch moderate Druckänderungen die Dichte in einem weiten Bereich variiert werden kann [75]. Dichtefluktuationen und isotherme Kompressibilität $\kappa_{\mathrm{T}}$ sind direkt proportional zueinander [76].

Die Existenz solcher Bereiche hoher und niedriger Dichte kann folgendermaßen veranschaulicht werden: Bringt man ein Teilchen in die Nähe eines anderen Teilchens und erhöht dadurch die Dichte in diesem Bereich, so ist dies mit einer Abnahme an Entropie verbunden. Dieser Entropieverlust wird durch den Energiegewinn kompensiert, der auftritt, wenn ein Teilchen von einem großen Abstand in den attraktiven Teil des Wechselwirkungspotentials gebracht wird [77. Solche Gebiete höherer Dichte werden auch als Cluster bezeichnet.

Die Inhomogenitäten wirken sich auch auf überkritische Lösungen aus. Überkritische Lösungen unendlicher Verdünnung werden üblicherweise nach der Art der von dem gelösten Teilchen induzierten Störungen in attraktive, schwach attraktive und repulsive Mischungen unterschieden [78, 79, 80. Qualitativ sind attraktive Lösungen dadurch gekennzeichnet, dass die mittlere lokale Dichte um das Gelöste $\rho_{12}$ (1: Lösungsmittel, 2: Gelöstes) größer als die mittlere lokale Dichte um ein Lösungsmittelmolekül $\rho_{11}$ ist: $\rho_{12}>\rho_{11}$. An dieser Stelle sei 
bemerkt, dass in einem überkritischen Fluid die mittlere lokale Dichte $\rho_{11}$ größer als die mittlere homogene Dichte $\rho$ sein kann, weil sich in einem solchen inhomogenen Fluid die Mehrheit der Lösungsmittelmoleküle in Bereichen höherer Dichte aufhält. In einer schwach attraktiven Mischung gilt $\rho_{11} \geq \rho_{12} \geq \rho$, was bedeutet, dass die mittlere lokale Dichte um das Gelöste kleiner als um ein Lösungsmittelmolekül ist, die mittlere homogene jedoch noch übertrifft. Für eine repulsive Mischung gilt nun, dass die mittlere lokale Dichte um das Gelöste geringer als die mittlere Lösungsmitteldichte ausfällt: $\rho_{12}<\rho$ [77]. Makroskopisch zeichnen sich attraktive Mischungen durch ein negatives partielles molares Volumen $\bar{v}_{2}^{\infty}$ (Verhalten des Volumens einer unendlich verdünnten Mischung bezüglich Zugabe von Gelöstem bei konstantem Druck und Temperatur), repulsive und schwach attraktive durch ein positives aus. Ebenso wie die isotherme Kompressibilität divergiert $\bar{v}_{2}^{\infty}$ am kritischen Punkt [80].

Die mittlere lokale Dichte um das Gelöste ist eine Funktion des thermodynamischen Zustands der Mischung. Die Beobachtung der lokalen Dichtevergrößerung einer attraktiven Mischung besteht oft darin, dass sich nach Druckänderung die mittlere lokale Dichte in einem anderen Maß als die homogene verändert. Experimentell wird dies durch die isotherme und dichteabhängige Messung einer auf die lokalen Dichte sensitiven Observablen, wie z. B. die Lösungsmittelverschiebung eines Absorptionsspektrums gegenüber dem Gasphasenspektrum, erreicht. Bei einer Auftragung der Observablen gegen die Dichte ergeben sich drei charakteristische Bereiche: Bei niedrigen Dichten steigt die Messgröße stark an, danach, bei Dichten um den kritischen Punkt, befindet sich eine Region relativer Invarianz, während anschließend die Messgröße wiederum stärker ansteigt. Durch die attraktiven Wechselwirkungen zwischen Gelöstem und Lösungsmittel wird im unteren Dichtebereich die schnelle Auffüllung der ersten Lösunsmittelschalen ermöglicht, während darauf folgende Druckerhöhungen die lokale Dichte kaum erhöhen bis zu dem Punkt, an dem die mittlere homogene Lösungsmitteldichte die lokale erreicht. Anschließende Druckerhöhungen lassen lokale und homogene Dichte gleichförmig anwachsen. Die lokale Dichtevergrößerung kann in der Regel nur bei reduzierten Temperaturen $\mathrm{T}_{\mathrm{r}} \leq 1.04$ beobachtet werden [77, 81]. Weitere anschauliche Größen sind neben der mittleren lokalen Dichte die relative lokale Dichte $\rho_{\text {rel }}=\rho_{12} / \rho$ sowie die lokale Dichtevergrößerung $\rho_{\mathrm{ex}}=\rho_{12}-\rho . \rho_{\mathrm{ex}}$ weist eine Nullstelle bei verschwindender und eine bei hoher Dichte auf, dazwischen, bei einer Dichte, die ungefähr 1/3 bis $1 / 2$ der kritischen Dichte $\rho_{\mathrm{c}}$ entspricht, wird diese Funktion maximal. Die relative lokale Dichte $\rho_{\text {rel }}$ hingegen fällt monoton von einem Anfangswert bei verschwindender Dichte auf eins ab [82, 83]. Die Dichteabhängigkeit aller drei Größen ist in Abb.2.9 veranschaulicht.

Strukturen in Lösung und damit die lokale Dichte lassen sich mit der PaarKorrelationsfunktion $g\left(\vec{r}=\overrightarrow{r_{2}}-\overrightarrow{r_{1}}\right)$ beschreiben. Die Paar-Korrelationsfunktion ist die auf eine vollständig zufällige Verteilung normierte Wahrscheinlichkeit zwei Teilchen an einem Ort $\overrightarrow{r_{1}}$ und $\overrightarrow{r_{2}}$ zu finden. Ist die Verteilung isotrop, so geht die Paar-Korrelationsfunktion in die radiale Verteilungsfunktion $g(r)$ über [84]. Die Paar-Korrelationsfunktion kann durch Monte-Carlo [85] oder molekulardynamische (MD) [86] Simulationen modelliert werden. Als Maß für die lokale Dichte kann die Koordinationszahl $Z=4 \pi n \int_{0}^{r_{\mathrm{m}}} g(r) r^{2} \mathrm{~d} r$, wobei $n$ : Teilchenzahldichte, $r_{\mathrm{m}}$ : erstes Minimum der Verteilungsfunktion [84] oder das Maximum der Verteilungsfunktion $g_{\max }$ [87] dienen. 



\section{Experimentelle Technik}

\subsection{Wahl der Methode}

Zur Untersuchung der Dynamik der Photodissoziation und Photoisomerisierung des Diiodmethans wird die Pump-Probe-Absorptionsspektroskopie verwendet. Bei dieser experimentellen Technik wird zuerst ein Pumppuls in die Probe eingestrahlt und anschließend nach einer definierten Verzögerungszeit die Absorption eines zweiten Pulses, des Probepulses, gemessen. Grundlage der Absorptionsspektroskopie ist das Lambert-Beersche Gesetz:

$$
O D=-\lg \frac{I}{I_{0}}=\varepsilon(\lambda) \cdot c \cdot l
$$

wobei $I_{0}$ und $I$ die Intensität vor und nach dem Durchgang durch das absorbierende Medium, $\varepsilon$ der molare dekadische Absorptionskoeffizient, $\lambda$ die Probewellenlänge, $c$ die Konzentration, $l$ die Länge des Mediums und $O D$ die optische Dichte sind.

Um die Dynamik der bei dem Käfigeffekt auftretenden Prozesse (Dissoziation, Isomerisierung und Schwingungsenergierelaxation) zu beobachten, ist eine Zeitauflösung in der Größenordnung von 100 fs erforderlich. Die dafür nötigen kurzen Pulse sind durch Titan:SaphirLasersysteme erhältlich.

In dieser Arbeit wurden zeitabhängige Absorptionen mit verschiedenen Probewellenlängen gemessen und daraus zeitaufgelöste Absorptionsspektren rekonstruiert. Zudem wurde die Abhängigkeit der Dynamik von der Anregungswellenlänge untersucht. Durch den Einsatz optisch parametrischer Verstärker lässt sich die dafür erforderliche Durchstimmbarkeit realisieren.

\subsection{Das Lasersystem im Überblick}

Das in dieser Arbeit verwendete Lasersystem wurde bereits ausführlich in der Literatur beschrieben [88, 89, 90, 91, 92, 93, 94] und wird deshalb hier nur kurz dargestellt. Der Aufbau des Lasersystems ist schematisch in Abb.3.1 aufgezeichnet. Als Laserlichtquelle dient ein kommerzieller, regenerativ verstärkter Titan:Saphir-Laser (Clark CPA-2001 95]), welcher Pulse mit einer Zentralwellenlänge von $773 \mathrm{~nm}$, einer Energie von $\sim 0.9 \mathrm{~mJ}$ und $150 \mathrm{fs}$ Dauer bei einer Repetitionsrate von $1 \mathrm{kHz}$ liefert. Ein Drittel der Laserintensität dient zur Erzeugung der Pumppulse im NOPA (siehe Abschn.3.3.2) und zwei Drittel zur Erzeugung der Probepulse im TOPAS (siehe Abschn.3.3.3).

Die Pulse aus dem NOPA zwischen 460 und $700 \mathrm{~nm}$ werden zeitlich komprimiert und frequenzverdoppelt, um eine Anregung zwischen 230 und $350 \mathrm{~nm}$ zu ermöglichen. Der TOPAS lässt sich computergesteuert zwischen 190 und $1090 \mathrm{~nm}$ durchstimmen. 


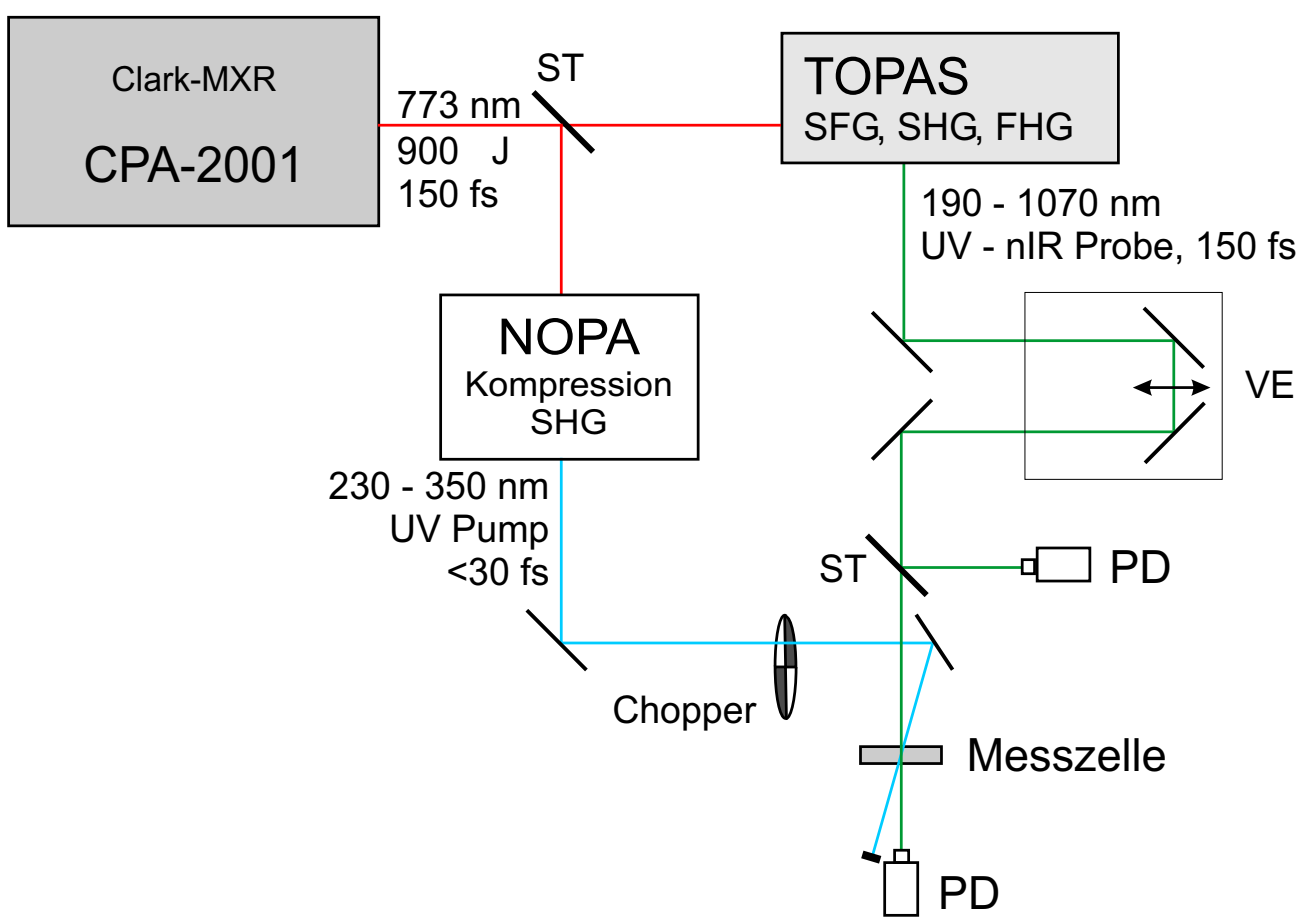

Abbildung 3.1: Das verwendete Lasersystem im Überblick.

Der Probestrahl wird über eine ebenfalls computergesteuerte Verschiebebühne geleitet, wodurch die Verzögerungszeit zwischen Pump- und Probestrahl mit einer Genauigkeit von $\sim 1.3 \mathrm{fs}$ eingestellt werden kann. Beide Strahlen kreuzen sich in der Probe unter einem kleinen Winkel. Die Zeitauflösung der Apparatur liegt zwischen 200 und 300 fs.

\subsection{Die Komponenten des Lasersystems}

\subsubsection{CPA-2001}

Bei dem CPA-2001 (chirped pulse amplifier [96]) handelt es sich um einen regenerativ verstärkten Ti:Sa-Laser. Die Verstärkung der sog. „Seedpulse“ erfolgt dabei in einem Laserresonator, dessen aktives Medium aus einem optisch gepumpten Ti:Sa-Kristall besteht. Ein gütegeschalteter frequenzverdoppelter Nd:YAG-Laser erzeugt die Besetzungsinversion mit einer Repetitionsrate von $1 \mathrm{kHz}$ während zeitverzögert dazu die zu verstärkenden Pulse in die Verstärkerkavität eingekoppelt werden. Diese „Seedpulse“ werden durch polarisationsadditive Modenkopplung [97] in einem Glasfaser-Ringoszillator [98] erzeugt. Auf die Erzeugung von ultrakurzen Pulsen durch Modenkopplung wird hier nicht näher eingegangen und auf die Literatur [99, 100, 101, 102, 103] verwiesen. Um eine Zerstörung von Optiken durch zu hohe Spitzenintensitäten zu vermeiden, werden die Pulse vor Einkopplung in die Verstärkerkavität zeitlich gestreckt. Nach üblicherweise vier bis sechs Resonatorumläufen und damit verbundenen Verstärkungen ist die maximale Pulsintensität erreicht und der Puls wird mit 
Hilfe einer Pockelszelle ausgekoppelt. Anschließende Kompression führt zu Pulsen mit einer typischen Länge von 150 fs 95 .

\subsubsection{NOPA}

Die Funktionsweise des NOPA (non collinear parametric amplifier) beruht auf dem parametrischen Prozess, einem 3-Wellenmischprozess [99]. Bei diesem Prozess wird ein Pumpphoton in ein Photon größerer Energie (Signal) und ein Photon geringerer Energie (Idler) aufgeteilt. Wie bei allen 3-Wellenmischprozessen gelten Energieerhaltung $\omega_{\mathrm{P}}=\omega_{\mathrm{S}}+\omega_{\mathrm{I}}$ und Impulserhaltung $\overrightarrow{k_{\mathrm{P}}}=\overrightarrow{k_{\mathrm{S}}}+\overrightarrow{k_{\mathrm{I}}}$. Der Winkel $\alpha$ zwischen den Wellenvektoren $\overrightarrow{k_{\mathrm{S}}}$ und $\overrightarrow{k_{\mathrm{I}}}$ wird dabei so gewählt, dass die Projektion der Gruppengeschwindigkeit des Idlerpulses $v_{\mathrm{g}, \mathrm{I}}$ auf seine Ausbreitungsrichtung gleich der Gruppengeschwindigkeit des Signalpulses $v_{\mathrm{g}, \mathrm{S}}$ ist [104, 105, 106]:

$$
v_{\mathrm{g}, \mathrm{S}}=\cos (\alpha) \cdot v_{\mathrm{g}, \mathrm{I}}
$$

Experimentell realisiert wird der parametrische Prozess, indem in einem BBO-Kristall die 2. Harmonische der Laserfundamentalen $(387 \mathrm{~nm})$, die hier als Pumppuls fungiert, mit einem gechirpten Weißlichtkontinuum überlagert wird. Über die Kristallverkippung und damit der Phasenanpassung sowie der Verzögerung zwischen Pumppuls und Weißlichtkontinuum lassen sich so Pulse im Bereich von 460-700 nm erzeugen. Nach anschließender Kompression und Frequenzverdopplung erhält man Pulse im Wellenlängenbereich von 230-350 nm mit einer Dauer von $\sim 30$ fs und einer Energie von $\sim 0.05 \mu \mathrm{J}$.

\subsubsection{TOPAS}

Ebenso wie beim NOPA handelt es sich beim TOPAS (travelling wave optical parametric amplifier of superfluorescence) (Lightconversion) um einen optisch parametrischen Verstärker. Im Gegensatz zum NOPA wird hier der bei hohen Intensitäten in einem nichtlinearen Kristall spontan auftretende parametrische Prozess (Superfluoreszenz) ausgenutzt. Dazu wird die Fundamentale in drei Komponenten aufgeteilt, wobei die ersten beiden zur Verstärkung und der dritte Teil zur Erzeugung der Superfluoreszenz dienen. Die im ersten Durchgang durch den nichtlinearen Kristall erzeugten kollinearen Anteile der Superfluoreszenz werden durch zwei weitere Durchgänge verstärkt bevor die für die folgenden parametrischen Prozesse benötigten Wellenlängen durch ein Gitter abgetrennt werden. Dieses Licht dient als „Seedpuls" für zwei weitere parametrische Prozesse, die durch die ersten beiden Komponenten der Laserfundamentalen gepumpt werden. Auf diese Art und Weise können Signalpulse im Wellenlängenbereich von 1070-1560 nm und Idlerpulse im Bereich von 1560-2790 nm erzeugt werden. Durch bis zu drei weitere Mischprozesse (zweite Harmonische von Signal bzw. Idler oder Summenfrequenz aus Fundamentale und Signal bzw. Idler) können Pulse im Bereich von 190-1070 nm erzeugt werden. Für den detaillierten Strahlengang und Funktionsweise sei auf [107, 108] verwiesen.

$\mathrm{Zu}$ Verringerung des Rauschens und zur Strahlaufweitung wird der Probestrahl mit einem Kepler-Teleskop $\left(f_{1}=80 \mathrm{~mm}, f_{2}=100 \mathrm{~mm}\right)$ durch ein $150 \mu \mathrm{m}$-Pinhole fokussiert. Dieser Aufbau wirkt wie ein Fourier-Kurzpass-Raumfilter 99. 


\subsubsection{Pump-Probe-Interferometer}

Der Probestrahl wird über eine Verschiebebühne (Physik Instrumente, PI M-415.DG) geführt und anschließend auf einen Strahlteiler geleitet. Die Intensität $I_{0}$ (siehe Gl.3.1) des dabei transmittierten Anteils wird von einer Photodiode (Hamamatsu, 1226-8BQ bzw. 13368BQ0A) gemessen während der reflektierte Anteil mit dem Pumpstrahl in der Probe interferiert. Dazu wird der reflektierte Strahl mit einem Parabolspiegel $(f=152.4 \mathrm{~mm})$ in die Probe fokussiert und eine dahinter angebrachte weitere Photodiode misst die Intensität $I$.

Ein vor der Frequenzverdopplung in den Strahlengang eingebrachter Chopper blockt jeden zweiten Pumppuls ab, so dass sich als tatsächlich Messgröße die Änderung der Absorption zwischen gepumpter und ungepumpter Messung ergibt:

$$
\triangle O D=O D_{\text {gepumpt }}-O D_{\text {ungepumpt }}
$$

Der Pumpstrahl wird mit einem zweiten Parabolspiegel $(f=203.3 \mathrm{~mm})$ in die Probe fokussiert, wobei beide Strahlen einen Winkel von $7^{\circ}$ einschließen. Es wird darauf geachtet, dass sich der Fokus des Pumpstrahls kurz vor dem des Probestrahls befindet, so dass der Durchmesser des Pumpstrahls am Ort des Überlapps leicht größer als der des Pumpstrahls ist.

Weil die bei der Photodissoziation von $\mathrm{CH}_{2} \mathrm{I}_{2}$ entstehenden Photoprodukte $\mathrm{I}_{2}$, das $\mathrm{CH}_{2} \mathrm{I}-$ Radikal und Ethan nicht durch das Pumplicht angeregt werden [29, 52], konnte eine stationäre Hochdruckzelle verwendet werden. Die Hochdruckzelle 92 besteht aus hochfestem Stahl (Inconel Alloy 718, Materialnr. 2.4969). Sie besitz ein Probevolumen von $\sim 2.6 \mathrm{~cm}^{3}$ und eine Schichtlänge von $2.5 \mathrm{~mm}$. Als Zellenfenster werden $2 \mathrm{~mm}$-Saphirfenster (Korth) mit optischer Achse in Strahlrichtung verwendet. Die Lösung lässt sich mit einem Magnetrührer umwälzen. Die Probensubstanz wird vor dem Zusammenbau der Zelle eingebracht und anschließend wird das Lösungsmittel mit wahlweise einer Spindelpresse oder einer HPLCPumpe (Techlab GmbH, Economy 2/ED) in die Zelle gedrückt. Die Temperatur wird mit einem Temperaturregler (Haake TP24) über ein Widerstandsthermometer (PT-100) konstant gehalten. Der Druck wird mit einem Piezomanometer (Keller GmbH, PA-8, Druckbereich 0-1000 bar) kontrolliert.

Mit einem Breitband- $\lambda / 2$-Plättchen kann die relative Polarisation von Pump- und Probestrahl auf den magischen Winkel, paralle oder senkrechte Polarisation gestellt werden.

\subsection{Anisotropie}

Bei Absorptions- oder Fluoreszenzspektroskopie mit hoher Zeitauflösung können Effekte der Molekülrotation beobachtet werden. Weil in diesem Experiment linear polarisiertes Licht verwendet wird, werden durch den Pumppuls nur Moleküle angeregt, deren Übergangsdipolmomente eine Komponente in Richtung des elektrischen Feldstärkevektors der einfallenden Welle haben. Durch den Anregungspuls erhält man demgemäß eine anisotrope Verteilung von angeregten Molekülen, die durch Rotation nach einer bestimmten Zeit in eine isotrope übergeht. Als Maß für die Anisotropie $r(t)$ dieser Verteilung wirkt die auf das Gesamtsignal 
normierte Differenz aus der Intensität eines Übergangs parallel $\left(I_{\|}\right)$und senkrecht $\left(I_{\perp}\right)$ zur Polarisation der Pumpwelle [109]:

$$
r(t)=\frac{I_{\|}-I_{\perp}}{I_{\|}+2 I_{\perp}}
$$

Bei folgender Betrachtung gehen wir davon aus, dass die Übergangsdipolmomente für Anregung und transiente Absorption parallel sind. Stellt man nun parallele Polarisation zwischen Pump- und Probestrahlen ein, so sieht man eine zeitliche Abnahme der Absorption durch Herauswandern der angeregten Moleküle aus dem räumlichen Beobachtungsfenster, während man bei Verwendung senkrechter Polarisation eine Zunahme der Absorption sieht. Messungen im magischen Winkel $\Theta_{M}$ sind hingegen frei von Effekten der Rotation. Zur Veranschaulichung des magischen Winkels geht man davon aus, dass der elektrische Feldvektor der Anregung einen Winkel $\Theta$ mit der $z$-Achse einschließt. So ist die Intensität für einen Übergang parallel zur $z$-Achse $I_{\|} \propto \cos ^{2} \Theta$ und für einen Übergang senkrecht dazu $I_{\perp} \propto \sin ^{2} \Theta$. Bei dem magischen Winkel gilt $I_{\perp}=2 I_{\|} \Longleftrightarrow \tan ^{2} \Theta_{\mathrm{M}}=2$, was einem Wert von $\Theta_{\mathrm{M}}=54.7^{\circ}$ entspricht [110].

Aus der Anfangsanisotropie $r(t=0)$ lässt sich der Winkel zwischen Übergangsdipolmomente von Anregung und Probe ermitteln. Dazu nimmt man an, dass das Übergangsdipolment der Anregung parallel zur z-Achse eines kartesischen Koordinatensystems orientiert ist $\left(I_{\|}=I_{z}\right)$. Ein einzelnes Dipolmonent des Probeübergangs schließt mit der $\mathrm{x}$-Achse den Winkel $\alpha$ mit der y-Achse den Winkel $\beta$ und mit der z-Achse den Winkel $\gamma$ ein. Für die Intensitäten entlang dieser Achsen gilt:

$$
\begin{aligned}
& I_{x} \propto \overline{\cos ^{2} \alpha} \\
& I_{y} \propto \overline{\cos ^{2} \beta} \\
& I_{z} \propto \overline{\cos ^{2} \gamma}
\end{aligned}
$$

wobei $\overline{\cos ^{2}}$ die Verteilung über die $\cos ^{2}$ der Winkel darstellt. Weil entlang der z-Achse angeregt wird ist die Verteilung der Übergangsdipolmomente um diese Achse symmetrisch: $I_{\perp}=I_{\mathrm{x}}=I_{\mathrm{y}}$. Mit

$$
r(t)=\frac{I_{\|}-I_{\perp}}{I_{\mathrm{x}}+I_{\mathrm{y}}+I_{\mathrm{z}}}
$$

und $\overline{\cos ^{2} \alpha}+\overline{\cos ^{2} \beta}+\overline{\cos ^{2} \gamma}=1$ ergibt sich:

$$
r(t)=\frac{3 \overline{\cos ^{2} \gamma}-1}{2}
$$

Mit Gleichung 3.9 und der Anfangsanisotropie ergibt sich der Winkel zwischen den Übergangsmomenten von Anregung und Probe. Setzt man $r(t)=0$, so erhält man für $\gamma$ ebenfalls den magischen Winkel. $r$ kann maximal den Wert 0.4 für parallele Polarisation von Anregung und Probe sowie -0.2 für senkrechte Polarisation annehmen [109]. 


\subsection{Anpassung der Daten}

Die Absorptionszeitprofile werden mit einer Modellfunktion angepasst, die sich aus einer Faltung von einer Exponentialfunktion mit einer Gaußfunktion ergibt:

$$
f(t)=\int_{0}^{\infty} g(t-\tau) \cdot e(\tau) \mathrm{d} \tau
$$

Die Gaussfunktion $g(t) 3.11$ hat eine der Kreuzkorrelation entsprechende Halbwertsbreite, während die Exponentialfunktion $e(t)$ aus einer Summe von abfallenden (3.12) und ansteigenden (3.13) Exponentialfunktionen besteht.

$$
\begin{aligned}
g(t) & =\frac{1}{\sqrt{2 \pi \sigma^{2}}} \exp \left(-\frac{t^{2}}{2 \sigma^{2}}\right) \\
e_{\mathrm{d}}(t) & =\exp \left(-\frac{t}{\tau_{\mathrm{d}}}\right) \\
e_{\mathrm{r}}(t) & =1-\exp \left(-\frac{t}{\tau_{\mathrm{r}}}\right)
\end{aligned}
$$

$\tau_{\mathrm{d}}$ und $\tau_{\mathrm{r}}$ sind dabei die Lebensdauern und $\sigma$ ist die halbe Halbwertsbreite der Gaußfunktion. Die analytische Faltung mit der Exponentialfunktion $e_{\mathrm{d}}$ ergibt

$$
f_{\mathrm{d}}(t)=\frac{1}{2} \exp \left(-\frac{2\left(t-t_{0}\right) \tau_{\mathrm{d}}-\sigma^{2}}{2 \tau_{\mathrm{d}}^{2}}\right)\left[\operatorname{erf}\left(\frac{\left(\left(t-t_{0}\right) \tau_{\mathrm{d}}-\sigma^{2}\right) \sqrt{2}}{2 \sigma \tau_{\mathrm{d}}}\right)+1\right],
$$

während die Faltung mit $e_{\mathrm{r}}$

$$
\begin{aligned}
f_{\mathrm{r}}(t)=\frac{1}{2}\left[\operatorname{erf}\left(\frac{\sqrt{2}\left(t-t_{0}\right)}{2 \sigma}\right) \exp \left(\frac{t-t_{0}}{\tau_{\mathrm{r}}}\right)-\exp \left(\frac{\sigma^{2}}{2 \tau_{\mathrm{r}}^{2}}\right) \operatorname{erf}\left(\frac{\left(\left(t-t_{0}\right) \tau_{\mathrm{r}}-\sigma^{2}\right) \sqrt{2}}{2 \sigma \tau_{\mathrm{r}}}\right)\right. \\
\left.+\exp \left(\frac{t-t_{0}}{\tau_{\mathrm{r}}}\right)-\exp \left(\frac{\sigma^{2}}{2 \tau_{\mathrm{r}}^{2}}\right)\right] \exp \left(-\frac{t-t_{0}}{\tau_{\mathrm{r}}}\right)
\end{aligned}
$$

ergibt. Der Parameter $t_{0}$ gibt den Zeitnullpunkt an. Desweiteren enthält die Funktion einen konstanten Endoffset $I_{0}$ :

$$
f_{0}(t)=I_{0} \int_{0}^{\infty} 1 \cdot g(t-\tau) \mathrm{d} \tau=\frac{1}{2} \operatorname{erf}\left(\frac{\sqrt{2}\left(t-t_{0}\right)}{2 \sigma}\right)+\frac{1}{2} I_{0} .
$$

Die Modellfunktion $f_{\mathrm{M}}$ ergibt sich nun als

$$
f_{\mathrm{M}}=\sum_{i} A_{i} f_{\mathrm{d}}\left(t, \tau_{\mathrm{d}, i}\right)+\sum_{j} B_{i} f_{\mathrm{r}}\left(t, \tau_{\mathrm{r}, j}\right)+f_{0}(t)+I_{1},
$$

wobei $A_{i}$ und $B_{j}$ die Amplituden und $I_{1}$ den Gesamtoffset darstellen. Die Anpassung der Daten erfolgt mit dem Levenberg-Marquardt Algorithmus, welcher in der Matlab Curve Fitting Toolbox 1.1 (Matlab Release 13) implementiert ist. 


\subsection{Verwendete Chemikalien}

Das verwendete Diiodmethan, sowie die benutzten Lösungsmittel und Gase sind mit Reinheits- und Herstellerangaben in Tabelle 3.1 aufgeführt. Alle Chemikalien wurden ohne weitere Reinigung verwendet.

\begin{tabular}{c|ccc} 
Substanz & Hersteller & Kurzform & Reinheit \\
\hline Diiodmethan zur Synthese & Merck-Schuchardt & $\mathrm{CH}_{2} \mathrm{I}_{2}$ & $>99 \%$ \\
Bromiodmethan & Aldrich & $\mathrm{CH}_{2} \mathrm{BrI}$ & - \\
Kohlendioxid & Messer Griesheim & $\mathrm{CO}_{2}$ & 4.5 \\
Fluoroform & Messer Griesheim & $\mathrm{CHF}_{3}$ & 4.5 \\
Ethan & Messer Griesheim & $\mathrm{C}_{2} \mathrm{H}_{6}$ & 2.5 \\
Xenon & Messer Griesheim & $\mathrm{Xe}$ & 4.0
\end{tabular}

Tabelle 3.1: Herkunft und Reinheit der verwendeten Chemikalien. 



\section{Experimentelle Ergebnisse}

\subsection{Stationäre Absorptionsspektren}

Zunächst wurden stationäre Absorptionsspektren des Diiodmethans in verschiedenen überkritischen Lösungsmitteln aufgenommen. Dazu stand ein Absorptionsspektrometer (Varian, Cary 5E) sowie eine beheizbare Hochdruckzelle (6 cm Schichtlänge, Volumen: $\left.4.7 \mathrm{~cm}^{3}\right)$ zur Verfügung. Abb.4.1zeigt die Absorptionsspektren von Diiodmethan in überkritischem Kohlendioxid $\left(35^{\circ} \mathrm{C}, 800\right.$ bar $)$, Fluoroform $\left(37^{\circ} \mathrm{C}, 800\right.$ bar $)$ und Ethan $\left(37^{\circ} \mathrm{C}, 800\right.$ bar $)$, jeweils bei bei einer Konzentration $c$ von $10^{-5}-10^{-4} \mathrm{~mol} / \mathrm{l}$. Zum Vergleich ist das Gasphasenspektrum aus [42] abgebildet. Es ergibt sich eine qualitative Übereinstimmung der Absorptionsspektren in Lösung mit dem Gasphasenspektrum, lediglich die $S_{2}$-Absorptionsbande um $250 \mathrm{~nm}$ ist in Lösung signifikant intensiver als in der Gasphase. Die Wellenlängen der Maxima der $S_{1}$-Bande werden in Tabelle 4.1 verglichen. Dabei zeigt sich, dass das Gasphasenspektrum und das Spektrum in Ethan nahezu identisch sind, während die Spektren in $\mathrm{CO}_{2}$ und $\mathrm{CHF}_{3}$ dagegen geringfügig blauverschoben sind. Die Blauverschiebung lässt sich durch eine Stabilisierung des Grundzustands durch das Dipolmoment des Fluoroforms und das Quadrupolmoment des Kohlendioxids erklären. Nach Absorption eines Photons ändert sich das Dipolmoments des Diiodmethans. Der Lösungsmittelcluster kann sich nach Anregung nicht umorientieren, so dass der $S_{1}$-Zustand nicht stabilisiert werden kann.

\begin{tabular}{c|c} 
& Abs.-Maximum/nm \\
\hline Gasphase [42] & 289.7 \\
Ethan & 290.0 \\
$\mathrm{CO}_{2}$ & 286.8 \\
$\mathrm{CHF}_{3}$ & 285.2
\end{tabular}

Tabelle 4.1: Vergleich der Absorptionsmaxima von $\mathrm{CH}_{2} \mathrm{I}_{2}$ in verschiedenen Lösungsmitteln.

Desweiteren konnte in dem hier untersuchten Druckbereich von 200-800 bar keine Dichteabhängigkeit der stationären Absorptionsspektren beobachtet werden.

\subsection{Zeitaufgelöste Absorptionsmessungen an $\mathrm{CH}_{2} \mathrm{I}_{2}$}

Bei allen in diesem Abschnitt dargestellten Messungen betrug die Konzentration an Diiodmethan 12-14 mmol/l. Um den Einfluss von Mehrphotonenprozessen, wie sie bei in üblichen Femtosekundenpulsen erreichten Intensitäten auftreten können, zu überprüfen, wurden Absorptionszeitprofile bei verschiedenen Pumpenergien aufgenommen. Der Kurvenverlauf der zeitaufgelösten Absorptionen zeigte keine Abhängigkeit von der Pumpintensität, so dass Mehrphotonenprozesse bei Anregung ausgeschlossen werden können [94. 


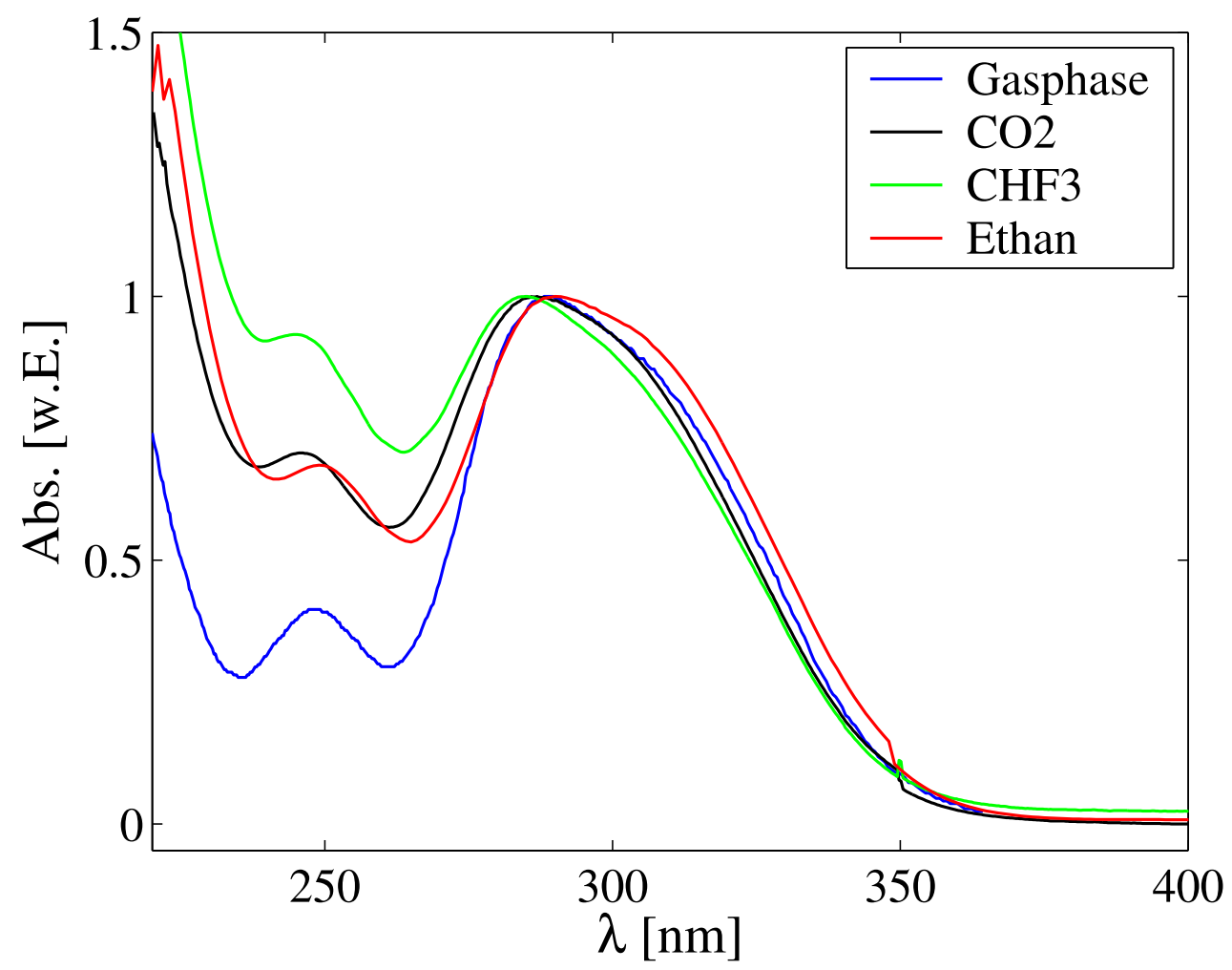

Abbildung 4.1: Vergleich der stationären Absorptionsspektren von $\mathrm{CH}_{2} \mathrm{I}_{2}$ in überkritischen Lösungsmitteln mit dem Gasphasenspektrum [42].

In Abb.4.2 ist ein typisches Absorptionszeitprofil in überkritischem Kohlendioxid dargestellt. Aufgetragen wird die Änderung der optischen Dichte $\triangle O D$ gegen die Zeit $t$. Die durchgezogene Linie stellt die Anpassung dar, als Modellfunktion wurde die Faltung einer Gaussfunktion mit einem einfach exponentiellen Abfall sowie einem einfach exponentiellen Anstieg verwendet (siehe Abschn. 3.5). Die Absorptionszeitprofile dieser Arbeit zeigen dieselben Charakteristika wie die aus vorhergehenden Untersuchungen bekannten Transienten [61, 111, 112]: einen Anstieg der Absorption innerhalb der Zeitauflösung, einen $200 \mathrm{fs}$ schnellen Abfall sowie einen mehrere Picosekunden langsamen Anstieg auf ein konstantes Endniveau. Eine Extrapolation des langsamen Anstiegs auf den Zeitnullpunkt ergibt eine positive, von Null verschiedene Absorption.

Die Bedingungen wurden so gewählt, dass die Temperatur $T$ mit $35^{\circ} \mathrm{C}$ knapp über der kritischen Temperatur $T_{\mathrm{c}}$ von $\mathrm{CO}_{2}$ lag (die kritischen Daten der verwendeten Lösungsmittel sind im Anhang A.1 aufgeführt). Dies entspricht einer reduzierten Temperatur $T_{\mathrm{r}}=T / T_{\mathrm{c}}=$ 1.01. Die Abfragewellenlänge liegt mit $370 \mathrm{~nm}$ im Maximum der UV-Absorptionsbande des Isomers (siehe Abb.2.7).

Ein sowohl qualitativ als auch quantitativ anderes Bild ergibt sich bei einem vergleichbaren Druck und einer Probewellenlänge von $435 \mathrm{~nm}$, was der roten Absorptionsflanke der UV-Bande entspricht (siehe Abb.4.3). Der der Bildung des Isomers zugeschriebene Anstieg ist deutlich schneller als im Maximum, zudem fällt die Absorption nach Erreichen des Maximums auf ein niedriger liegendes, konstantes Endniveau ab. Die Modellfunktion wird 

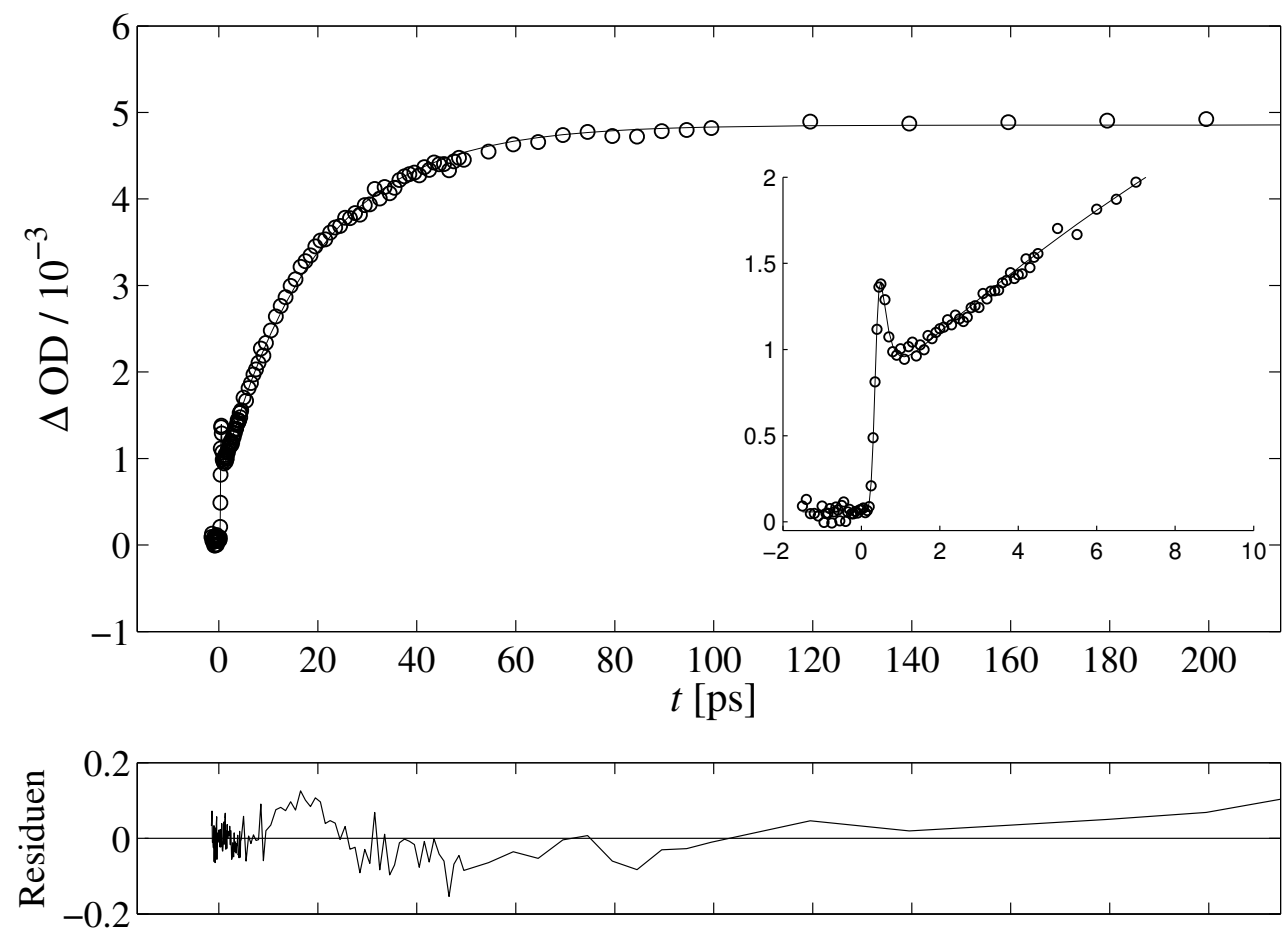

Abbildung 4.2: Absorptionszeitprofil von $\mathrm{CH}_{2} \mathrm{I}_{2}$ in $\mathrm{CO}_{2}\left(515\right.$ bar, $\left.35^{\circ} \mathrm{C}\right)$ bei $304 \mathrm{~nm}$ Anregung und $370 \mathrm{~nm}$ Abfrage.
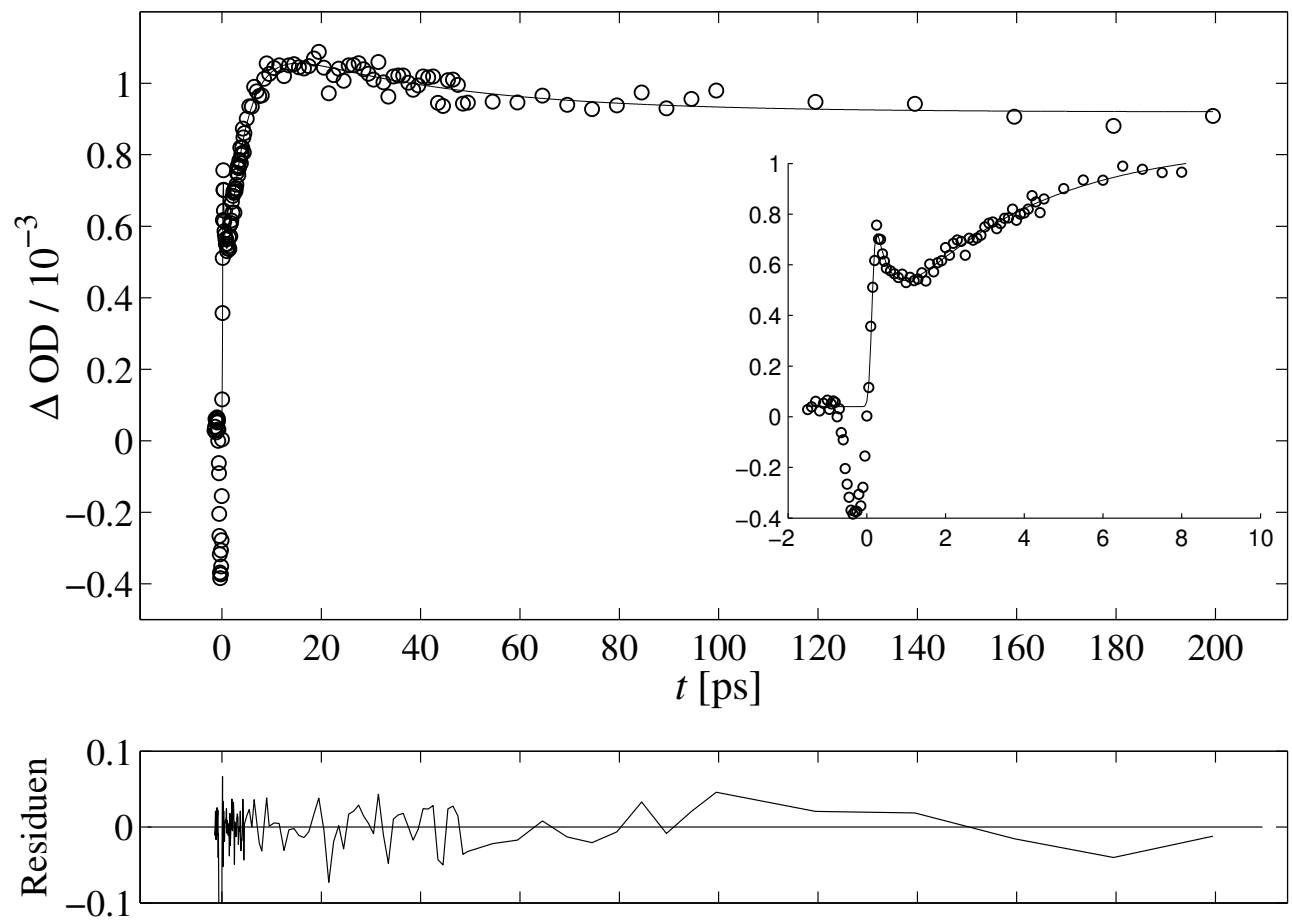

Abbildung 4.3: Absorptionszeitprofil von $\mathrm{CH}_{2} \mathrm{I}_{2}$ in $\mathrm{CO}_{2}\left(566\right.$ bar, $\left.35^{\circ} \mathrm{C}\right)$ bei $304 \mathrm{~nm}$ Anregung und $435 \mathrm{~nm}$ Abfrage. 
dementsprechend bei dieser Probewellenlänge um eine weitere, exponentiell abfallende Funktion ergänzt. Weiterhin zeigt diese Messung um den Zeitnullpunkt herum ein durch stark negative Absorption gekennzeichnetes kohärentes Artefakt. Solche Artefakte ließen sich in der Regel durch Justage vermeiden.

Die Absorptionszeitprofile hängen somit im starken Maße von der Probewellenlänge ab. Um ein vollständiges Bild dieser Wellenlängenabhängigkeit zu erhalten, wurden Transienten bei verschiedenen Probewellenlängen aufgenommen und daraus zeitaufgelöste Absorptionsspektren rekonstruiert.

\subsubsection{Probewellenlängenabhängigkeit}

Es wurden Absorptionszeitprofile im Bereich von 345 bis $455 \mathrm{~nm}$ in $10 \mathrm{~nm}$-Schritten aufgenommen. Dabei wurde gegebenenfalls vor jeder Messung der Überlapp zwischen Pump- und Probestrahl optimiert. Messungen bei kürzeren Wellenlängen waren wegen der Absorption des Muttermoleküls nicht möglich. Zudem sind Wellenlängen um die zweite Harmonische der Laserfundamentalen mit dem TOPAS nicht zugänglich. Zur Rekonstruktion der Spektren aus den gemessenen Absorptionszeitprofilen wurden diese zunächst durch die geeignete Modellfunktion angepasst. Anschließend wurde aus diesen Anpassungen zu verschiedenen Zeitpunkten die Signalintensität entnommen und gegen die Wellenlänge aufgetragen. Ein solches typisches zeitaufgelöstes Absorptionsspektrum in $\mathrm{CO}_{2}$ bei $300 \mathrm{~nm}$ Anregung zeigt Abb.4.4. Die in dieser Arbeit gemessenen transienten Spektren sind in ihrer Gestalt denen von Akesson et al. [61,63] ähnlich und zeigen ein zeitliches Anwachsen der Absorptionsbande sowie eine spektrale Verschmälerung zu längeren Verzögerungszeiten. Um die Probewellenlänge von $355 \mathrm{~nm}$ zeigt sich zu frühen Verzögerungszeiten ein lokales Maximum, zu längeren Verzögerungszeiten ist dieses Maximum schwächer ausgeprägt. Diese Struktur kann der Absorption des nach Photoanregung gebildeten $\mathrm{CH}_{2}$ I-Radikals zugeordnet werden (das Absorptionsspektrum von $\mathrm{CH}_{2} \mathrm{I}$ weist um $340 \mathrm{~nm}$ ein Maximum auf, vergl. Abb. 2.6).

Im Folgenden soll auf die spektrale Verschmälerung genauer eingegangen werden. Unter der Annahme, dass nach längeren Verzögerungszeiten ( $\geq 2$ ps) nur das Isomer zur Absorption beiträgt, lassen sich die Spektren zu den verschiedenen Verzögerungszeiten auf die Fläche unterhalb der Spektren normieren. Diese Fläche ist dabei proportional zur Konzentration an Isomer. Eine Normierung auf die Fläche der Spektren entspricht somit einer Normierung auf die Isomerkonzentration. Jedoch ist die Normierung der Spektren wegen ihrer Unvollständigkeit, insbesonders an der blauen Flanke, fehlerbehaftet. Abb.4.5 zeigt die Normierung des darüber dargestellten Spektrums. Die spektrale Verschmälerung ist in dieser Auftragung besonders deutlich: Zu frühen Verzögerungszeiten hat das Spektrum eine breite und flache Form, zu längeren Verzögerungszeiten wächst das Spektrum in der Mitte der Bande an, während es an den Flanken an Intensität verliert. Dies ist ein charakteristisches Verhalten für die Abkühlung schwingungsheißer Moleküle [113]. Im Bereich um $405 \mathrm{~nm}$ kreuzen sich die Spektren in einem quasi-isosbestischen Punkt. Der quasi-isosbestische Punkt (oder die quasi-isosbestische Region) bezeichnet in diesem Zusammenhang eine Wellenlänge, bei der der Extinktionskoeffizient nicht von der inneren Energie des Moleküls abhängt. Solche quasiisosbestischen Punkte sind z.B. bei heißen Absorptionsspektren aus Stoßwellenexperimenten zu finden [114, 115]. 


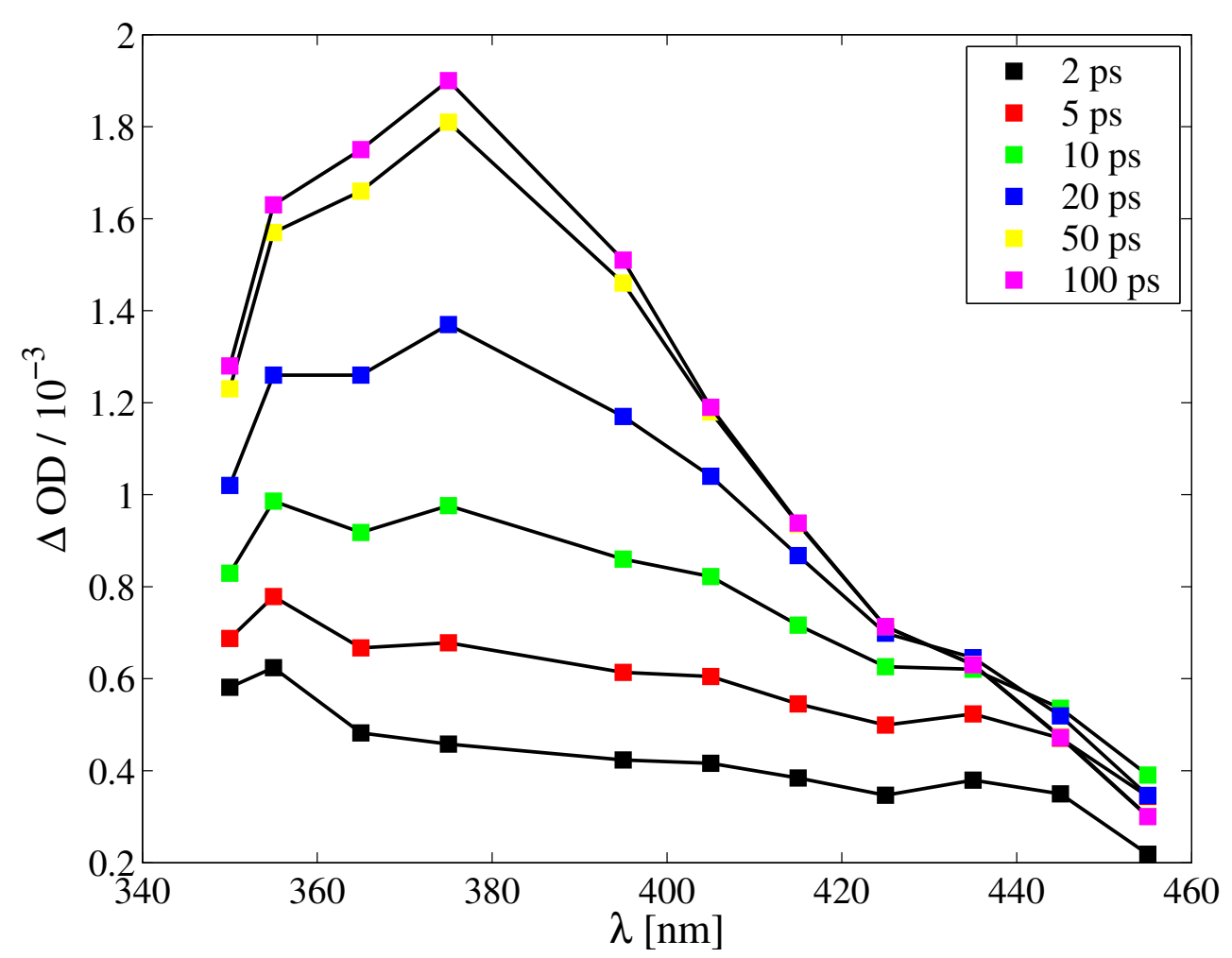

Abbildung 4.4: Rekonstruiertes zeitaufgelöstes Spektrum von $\mathrm{CH}_{2} \mathrm{I}_{2}$ in $\mathrm{CO}_{2}\left(400 \mathrm{bar}, 35^{\circ} \mathrm{C}\right)$.

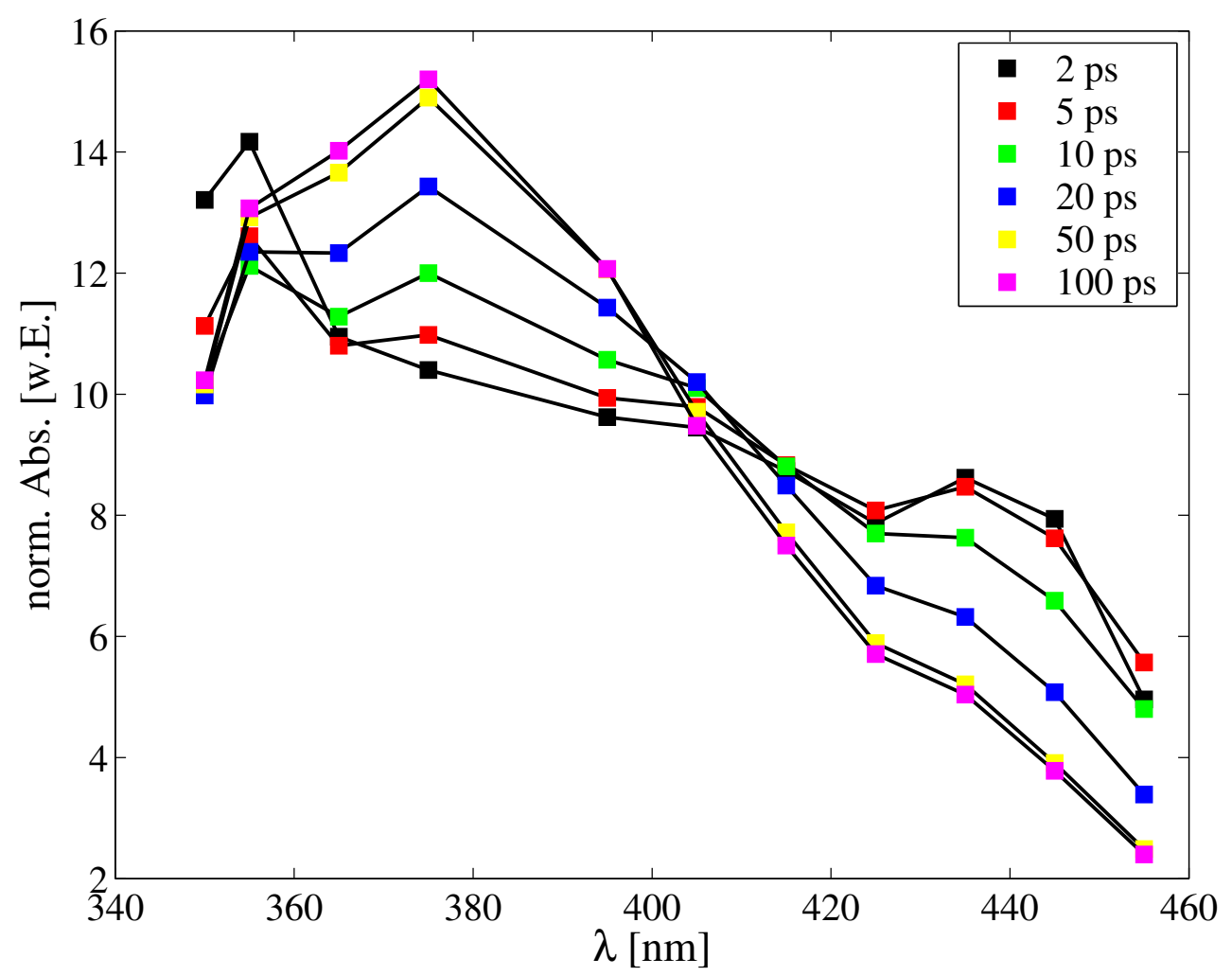

Abbildung 4.5: Rekonstruiertes und normiertes zeitaufgelöstes Spektrum von $\mathrm{CH}_{2} \mathrm{I}_{2}$ in $\mathrm{CO}_{2}$ (400 bar, $35^{\circ} \mathrm{C}$ ). 


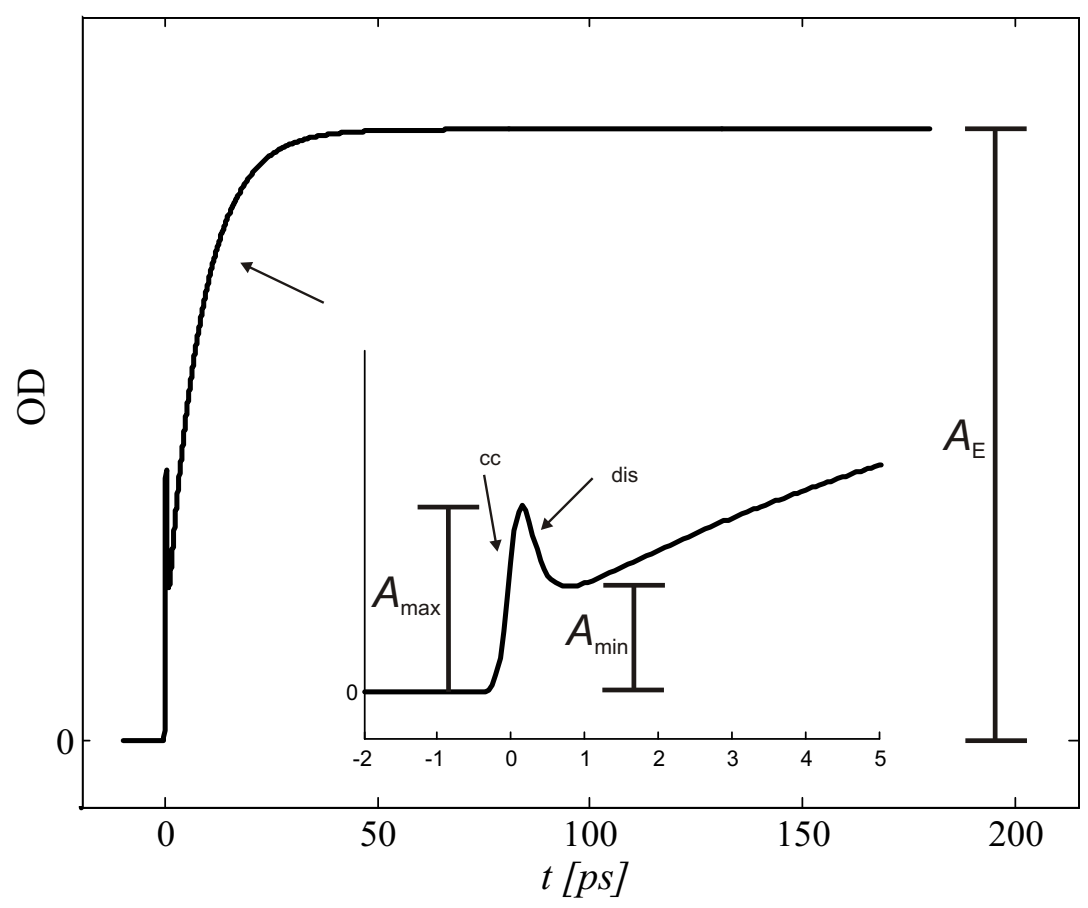

Abbildung 4.6: Schematisches Absorptionszeitprofil zur Veranschaulichung der charakteristischen Größen $\tau_{\text {cc }}, \tau, \tau_{\text {dis }}, A_{\max }, A_{\min }$ und $A_{\mathrm{E}}$.

Die Absorptionszeitprofile zu längeren Verzögerungszeiten sind dementsprechend nicht nur eine Folge der Populationszunahme an $\mathrm{CH}_{2} \mathrm{I}-\mathrm{I}$, sondern spiegeln auch die spektrale Evolution wider. Weil sich am quasi-isosbestischen Punkt keine spektrale Dynamik als Folge von Schwingungsenergietransfer beobachten lässt wird angenommen, dass bei dieser Probewellenlänge die reine Populationsänderung des Isomers beobachtet wird. Dichteabhängige Untersuchungen wurden aus diesem Grund hauptsächlich an den quasi-isosbestischen Punkten durchgeführt.

\subsubsection{Dichteabhängigkeit}

Eine der Hauptfragestellungen der vorliegenden Arbeit ist die Dichteabhängigkeit der Dissoziations- und Isomerisierungsdynamik. Zunächst wird in Abb.4.6 ein schematisches Absorptionszeitprofil dargestellt. Das Profil lässt sich mit der Kreuzkorrelation $\tau_{\text {cc }}$, der Lebensdauer des schnellen Abfalls $\tau_{\text {dis }}$ und des langsamen Anstiegs $\tau$ beschreiben. Zusätzlich sind zur vollständigen Beschreibung noch die Amplituden der Anfangsabsorption $A_{\max }$, der Minimumsabsorptions $A_{\text {min }}$ und der Endabsorption $A_{\mathrm{E}}$ nötig.

Abb. 4.7 zeigt Absorptionszeitprofile von $\mathrm{CH}_{2} \mathrm{I}_{2}$ in $\mathrm{CO}_{2}$ bei drei verschiedenen Lösungsmitteldrücken. Dabei lassen sich zwei Effekte erkennen: Alle drei Amplituden steigen mit zunehmender Dichte an und die langsame Anstiegszeit verkürzt sich. Im Folgenden wird die Dichteabhängigkeit der Absorptionszeitprofile, d.h. die Dichteabhängigkeit der angepassten Lebensdauern und Amplituden, dargestellt. 


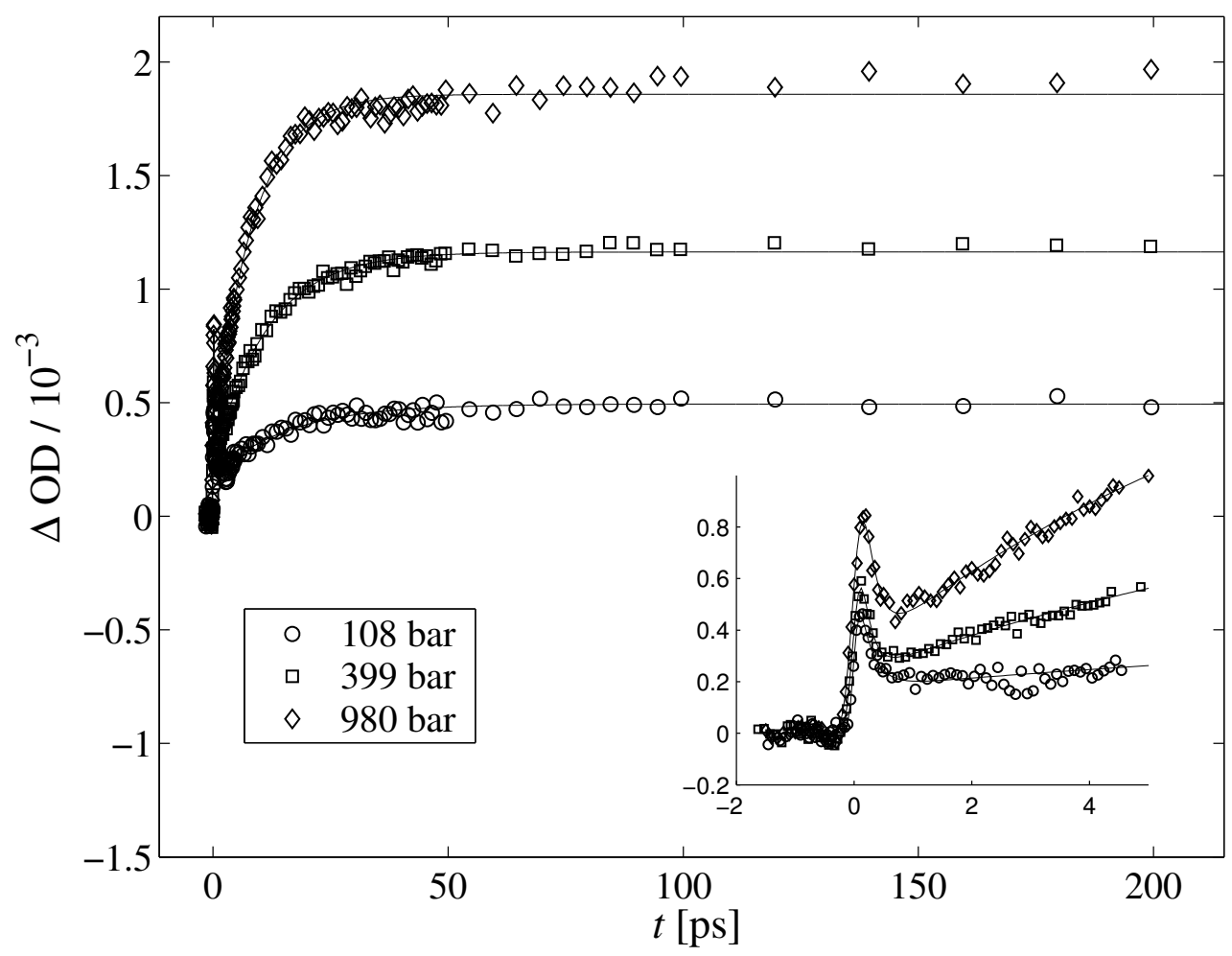

Abbildung 4.7: Druckabhängige Messungen in $\mathrm{CO}_{2}\left(35^{\circ} \mathrm{C}\right)$ bei $304 \mathrm{~nm}$ Anregung und $405 \mathrm{~nm}$ Abfrage.

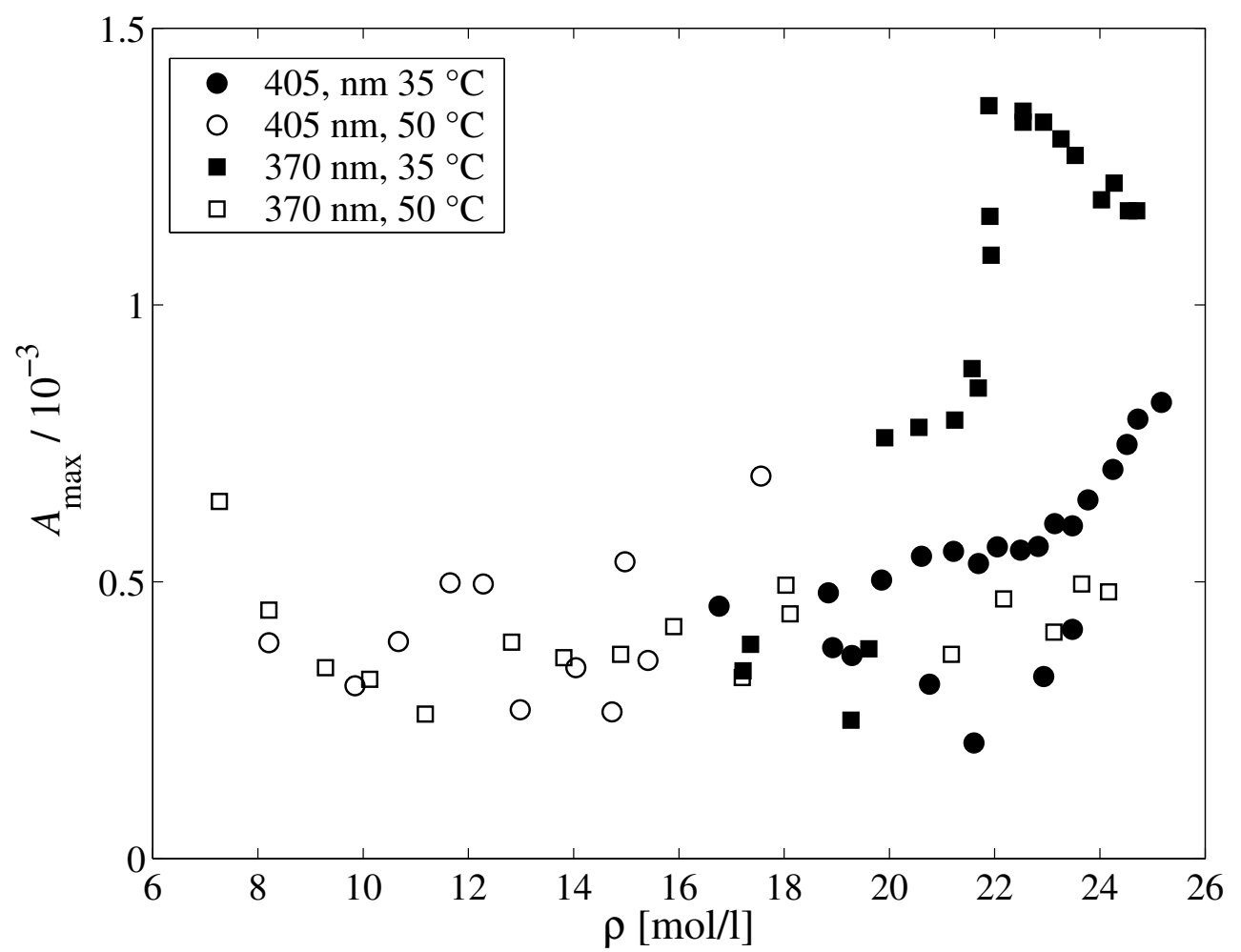

Abbildung 4.8: Auftragung des Anfangsmaximums $A_{\max }$ gegen die Lösungsmitteldichte von $\mathrm{CO}_{2}$ bei $304 \mathrm{~nm}$ Anregung und 370 bzw. $405 \mathrm{~nm}$ Abfrage. 
Abb.4.9 zeigt die aus den Modellfunktionen erhaltenen Endamplituden $A_{\mathrm{E}}$ in Abhängigkeit von der Dichte $\rho$. Die Daten für die Umrechnung von der Druck- in eine Dichteskala wurden dem NIST Chemistry WebBook [116] entnommen. Wegen der geringen Löslichkeit des Diiodmethans bei niedrigen Dichten $(<120$ bar $)$ wurde in diesem Bereich die Temperatur von 35 auf $50^{\circ} \mathrm{C}\left(T_{\mathrm{r}}=1.06\right)$ erhöht. Erst bei dieser Temperatur konnte die Konzentration an gelöstem Diiodmethan so weit erhöht werden, dass der niedrige Dichtebereich experimentell zugänglich wurde. Erklärbar ist dies durch eine Erhöhung des Dampfdrucks oder eine bessere Löslichkeit. Eine weitere Temperaturerhöhung führte jedoch zur Ablagerung des Diiodmethans auf den Fensterflächen, was zu einer starken Absorption des Probestrahls führte. Der niedrigste in dieser Arbeit erreichte Druck lag bei 94 bar und $50^{\circ} \mathrm{C}$, was einer reduzierten Dichte von $\rho_{\mathrm{r}}=0.68$ entspricht.

Weil zu langen Verzögerungszeiten nur das Isomer zur Absorption beiträgt, ist die Endamplitude $A_{\mathrm{E}}$ direkt proportional zur Quantenausbeute an Isomer $\Phi_{\text {iso }}$. In Abb.4.9 ergibt sich bei 370 und $405 \mathrm{~nm}$ Abfrage eine stark nichtlineare Abhängigkeit der Endamplitude $A_{\mathrm{E}}$ von der Dichte.

Wie in der Vergrößerung von Abb.4.7 deutlich zu erkennen, zeigt die Absorption des Anfangsmaximum $A_{\max }$ ebenfalls eine Dichteabhängigkeit, welche in Abb.4.8 dargestellt ist. In dieser Auftragung ergeben sich bei der Meßreihe von $370 \mathrm{~nm}$ Abfrage Abweichungen von monoton ansteigendem Verhalten. Wie in Abschn.2.2.2 beschrieben, wird die Absorption zu diesem Zeitpunkt der transienten Absorption aus dem ersten elektronisch angeregten Zustand des Muttermoleküls zugeordnet. Die Intensität dieser Absorption sollte nur proportional zu der Zahl an angeregten $\mathrm{CH}_{2} \mathrm{I}_{2}$-Molekülen sein und keine Dichteabhängigkeit aufweisen. Die Unstetigkeiten der Anfangsmaxima sind demnach eine Folge von Schwankungen der Justage und Pumpintensität sowie von Löslichkeitseffekten. Um diese Schwankungen auszugleichen, wurden in Abb.4.10 die Endamplituden auf das jeweilige Anfangsmaximum normiert: $A_{\mathrm{E}, \mathrm{n}}=A_{\mathrm{E}} / A_{\max }$. Die bei $35^{\circ} \mathrm{C}$ und $370 \mathrm{~nm}$ Abfrage auftretende Unstetigkeitsstelle wurde durch die Normierung eliminiert. Ein weiterer Hinweis auf die Zulässigkeit der Normierung ist die Unabhängigkeit der Messungen von der Pumpintensität, insbesondere zu frühen Verzögerungszeiten, wodurch kohärente Artefakte ausgeschlossen werden können. Die Dichtabhängigkeit von $\Phi_{\text {iso }}$ spiegelt sich demnach nicht in der Dichteabhängigkeit von $A_{\mathrm{E}}$, sondern in der von $A_{\mathrm{E}, \mathrm{n}}$ wider.

In Abb.4.10 erreichen die normierten Endamplituden bei $405 \mathrm{~nm}$ Abfrage und einer Dichte $>22 \mathrm{~mol} / \mathrm{l}$ einen konstanten Wert. Hierbei handelt es sich wahrscheinlich um ein Artefakt, weil weder die Messungen bei der Abfragewellenlänge von $370 \mathrm{~nm}$ noch die absoluten Endamplituden einen konstanten Wert erreichen. An dieser Stelle sei darauf hingewiesen, dass selbst bei den niedrigsten in dieser Arbeit erreichten Dichten der langsame Anstieg sichtbar ist, was bedeutet, dass selbst unter diesen Bedingungen noch Isomer gebildet wird.

Wie bereits festgestellt, fällt die Absorption nach mehreren Hundert Femtosekunden auf ein lokales Minimum ab. In Abb.4.11 sind die ebenfalls aus den Modellfunktionen erhaltenen Minima $A_{\text {min }}$ aufgetragen. Darunter, in Abb.4.12, sind die wiederum auf das Anfangsmaximum normierten Minima $A_{\text {min,n }}$ dargestellt. Wie bei den Endamplituden wird durch die Normierung eine Unstetigkeitsstelle eliminert. Trotz des geringen Signal-Rausch-Verhältnisses lassen sich zwei Trends erkennen: Bei beiden Temperaturen und Wellenlängen steigt das Minimum mit zunehmender Dichte nahezu linear an. Zudem liegen die Minima bei $50^{\circ} \mathrm{C}$ über denen von $35^{\circ} \mathrm{C}$. 


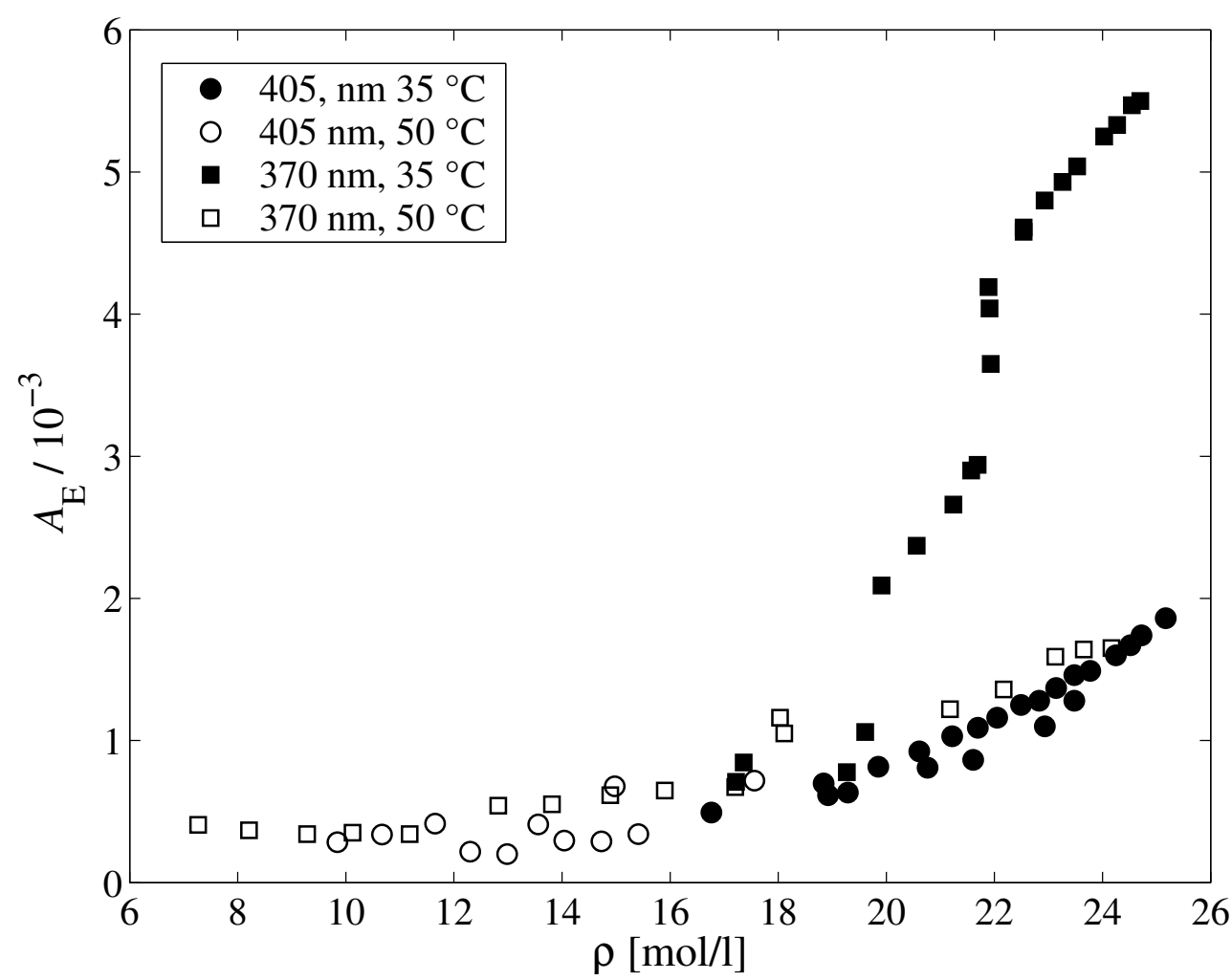

Abbildung 4.9: Auftragung der Endamplitude $A_{\mathrm{E}}$ gegen die Lösungsmitteldichte von $\mathrm{CO}_{2}$ bei $304 \mathrm{~nm}$ Anregung und 370 bzw. $405 \mathrm{~nm}$ Abfrage.

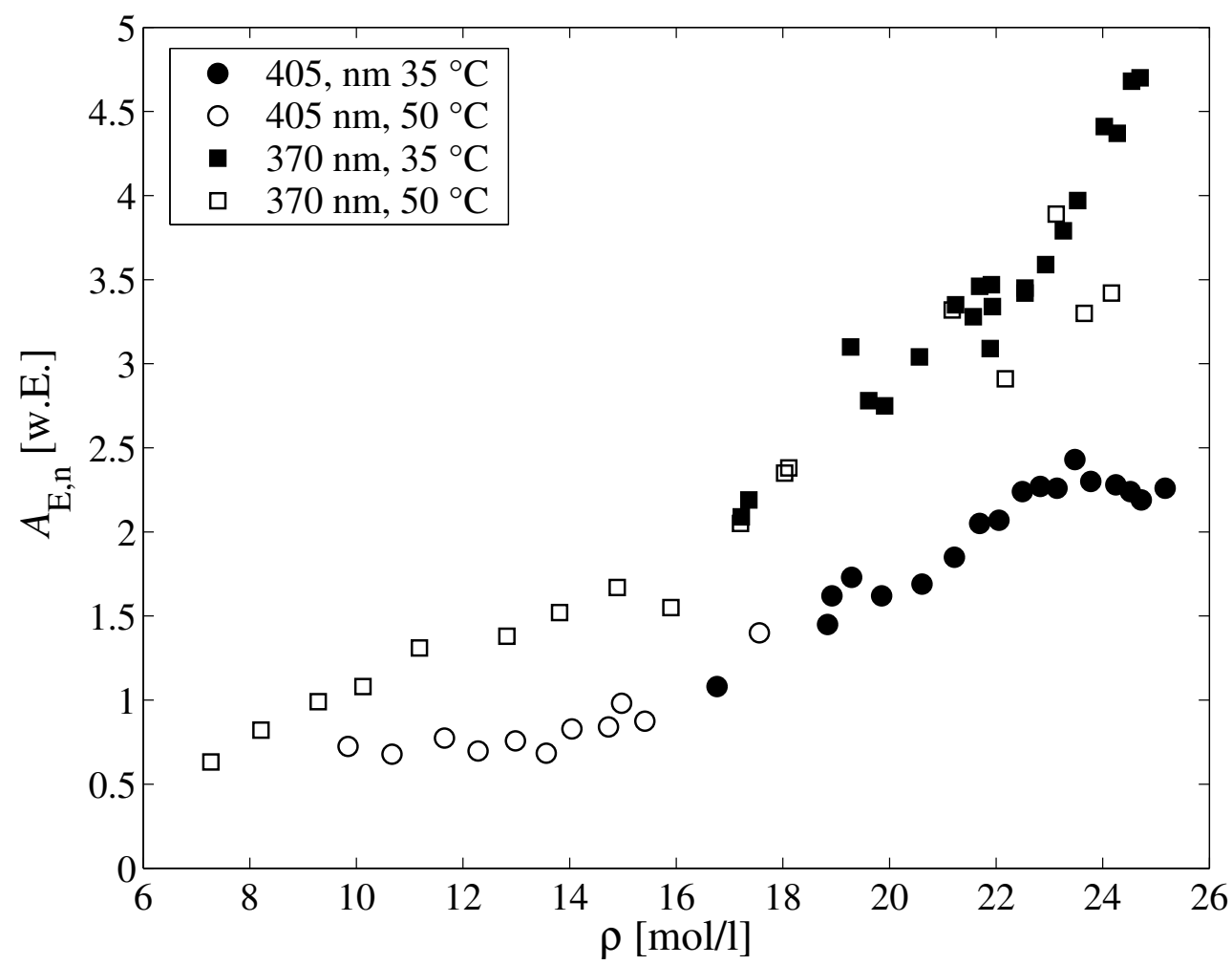

Abbildung 4.10: Auftragung der auf den Anfangspeak normierten Endamplitude $A_{\mathrm{E}, \mathrm{n}}$ gegen die Lösungsmitteldichte von $\mathrm{CO}_{2}$ bei $304 \mathrm{~nm}$ Anregung und 370 bzw. $405 \mathrm{~nm}$ Abfrage. 


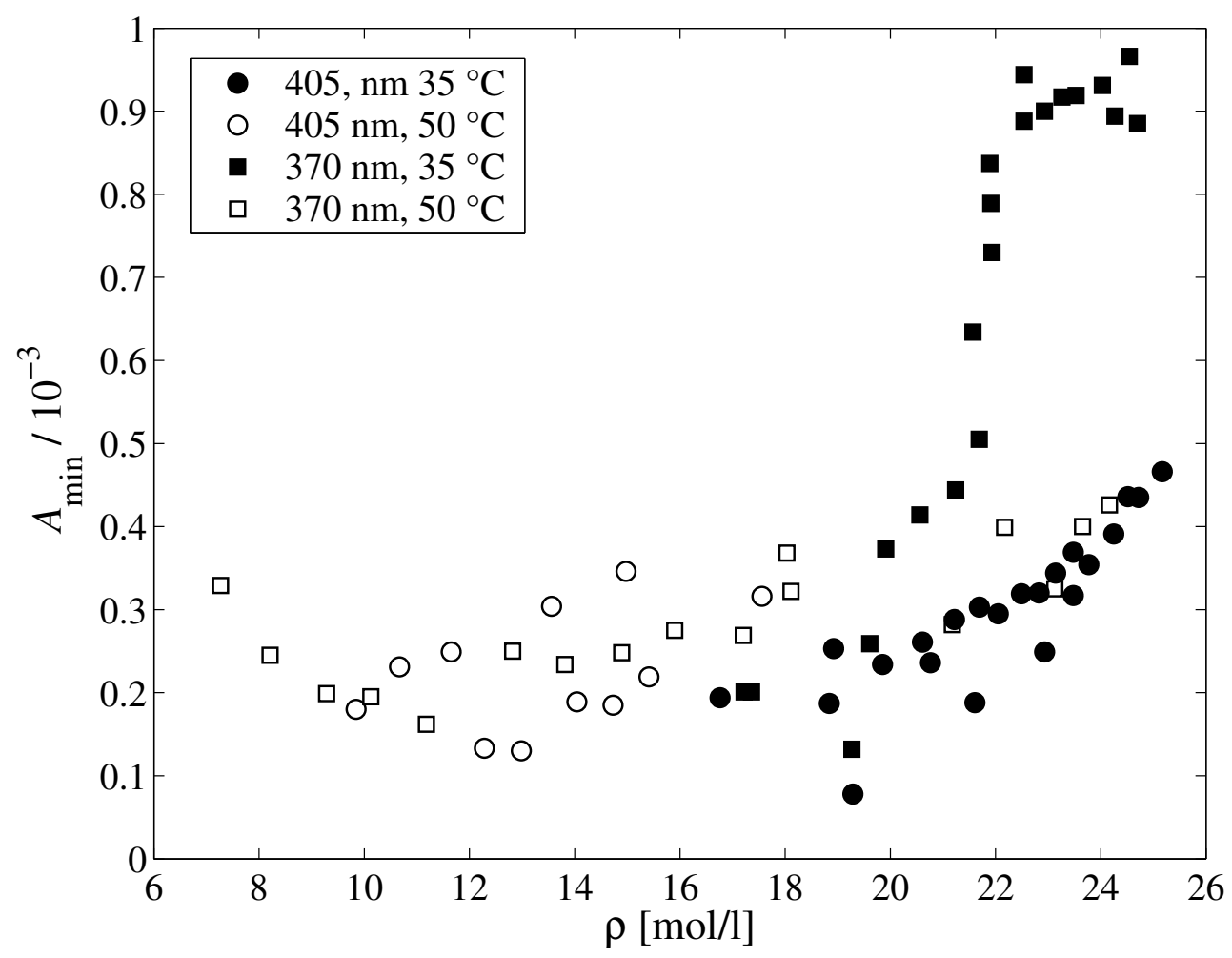

Abbildung 4.11: Auftragung des Minimums $A_{\text {min }}$ gegen die Lösungsmitteldichte von $\mathrm{CO}_{2}$ bei $304 \mathrm{~nm}$ Anregung und 370 bzw. $405 \mathrm{~nm}$ Abfrage.

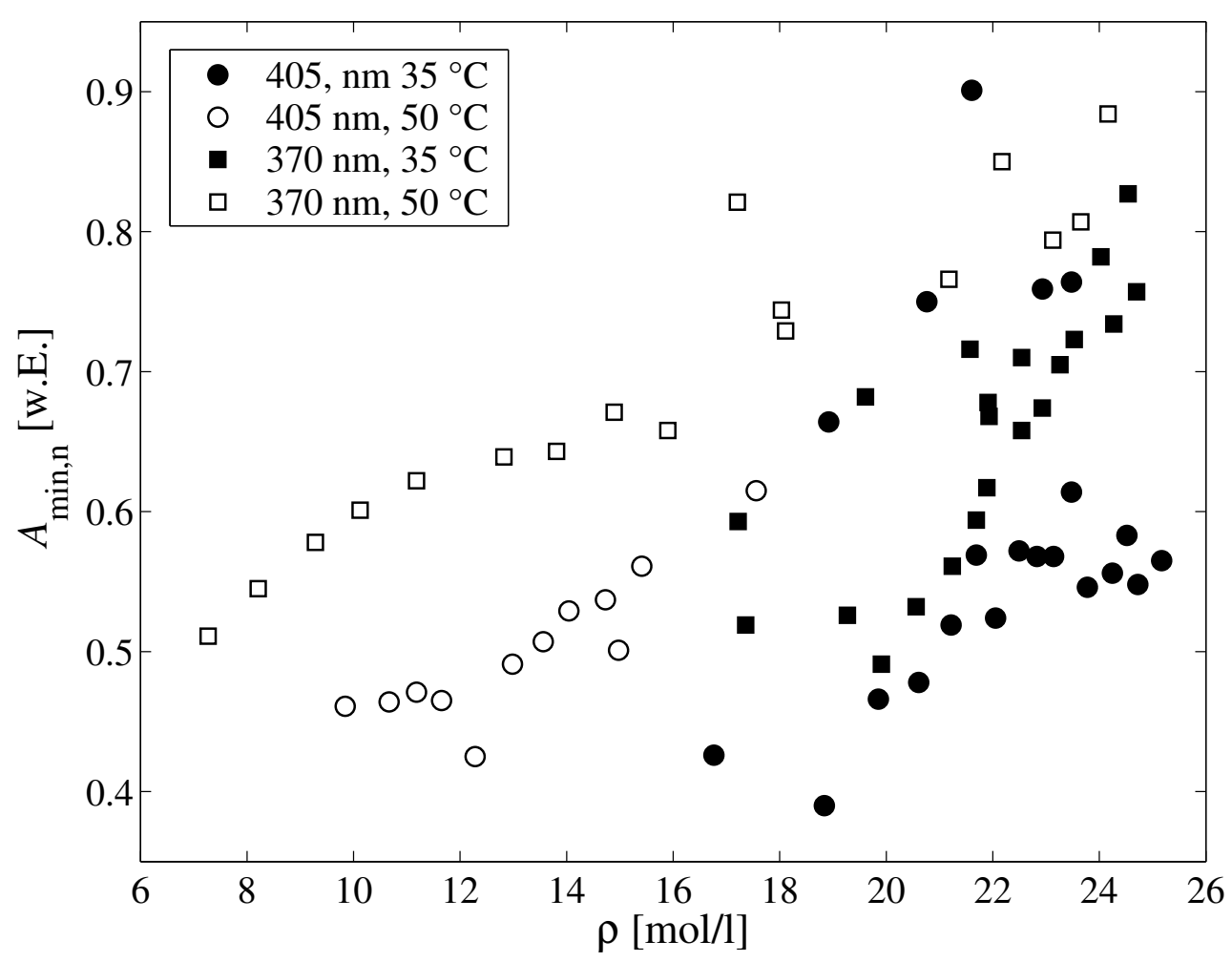

Abbildung 4.12: Auftragung des normierten Minimums $A_{\min , \mathrm{n}}$ gegen die Lösungsmitteldichte von $\mathrm{CO}_{2}$ bei $304 \mathrm{~nm}$ Anregung und 370 bzw. $405 \mathrm{~nm}$ Abfrage. 


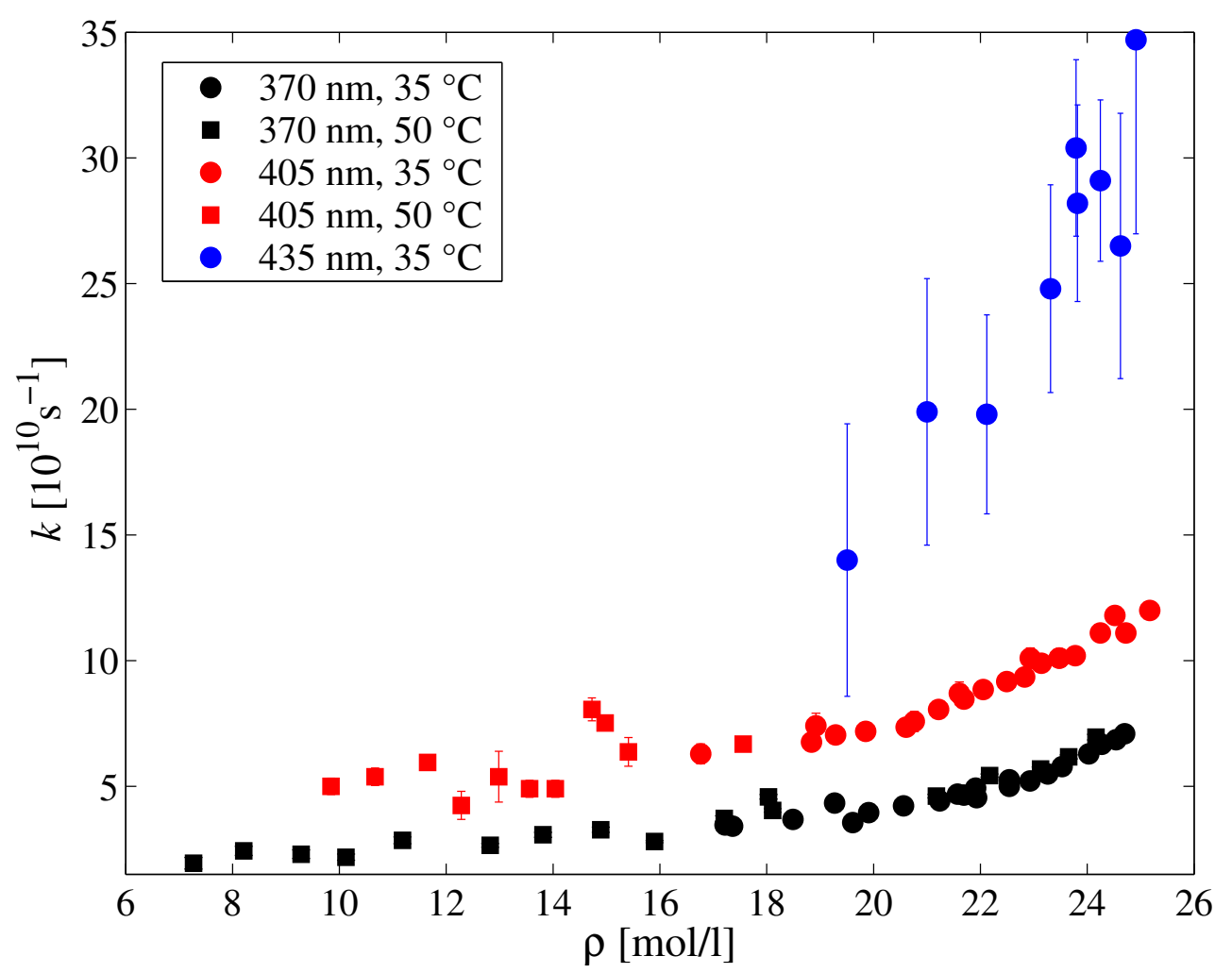

Abbildung 4.13: Auftragung von $k$ gegen die Lösungsmitteldichte von $\mathrm{CO}_{2}\left(35^{\circ} \mathrm{C}\right)$ bei $304 \mathrm{~nm}$ Anregung und 370, 405 bzw. 435 nm Abfrage.

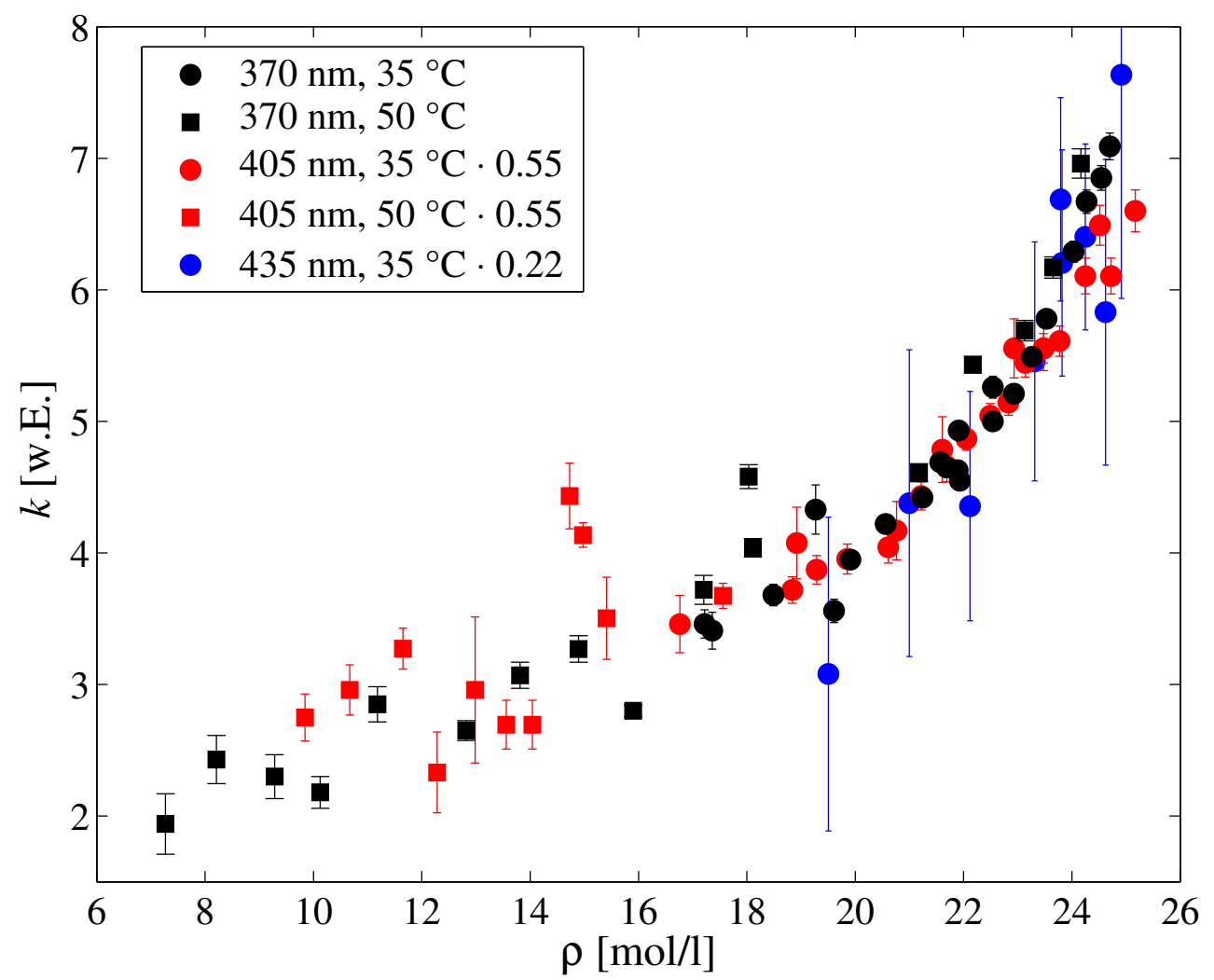

Abbildung 4.14: Dichteabhängigkeit von $k$ bei 370, 405 bzw. 435 nm Abfrage. 


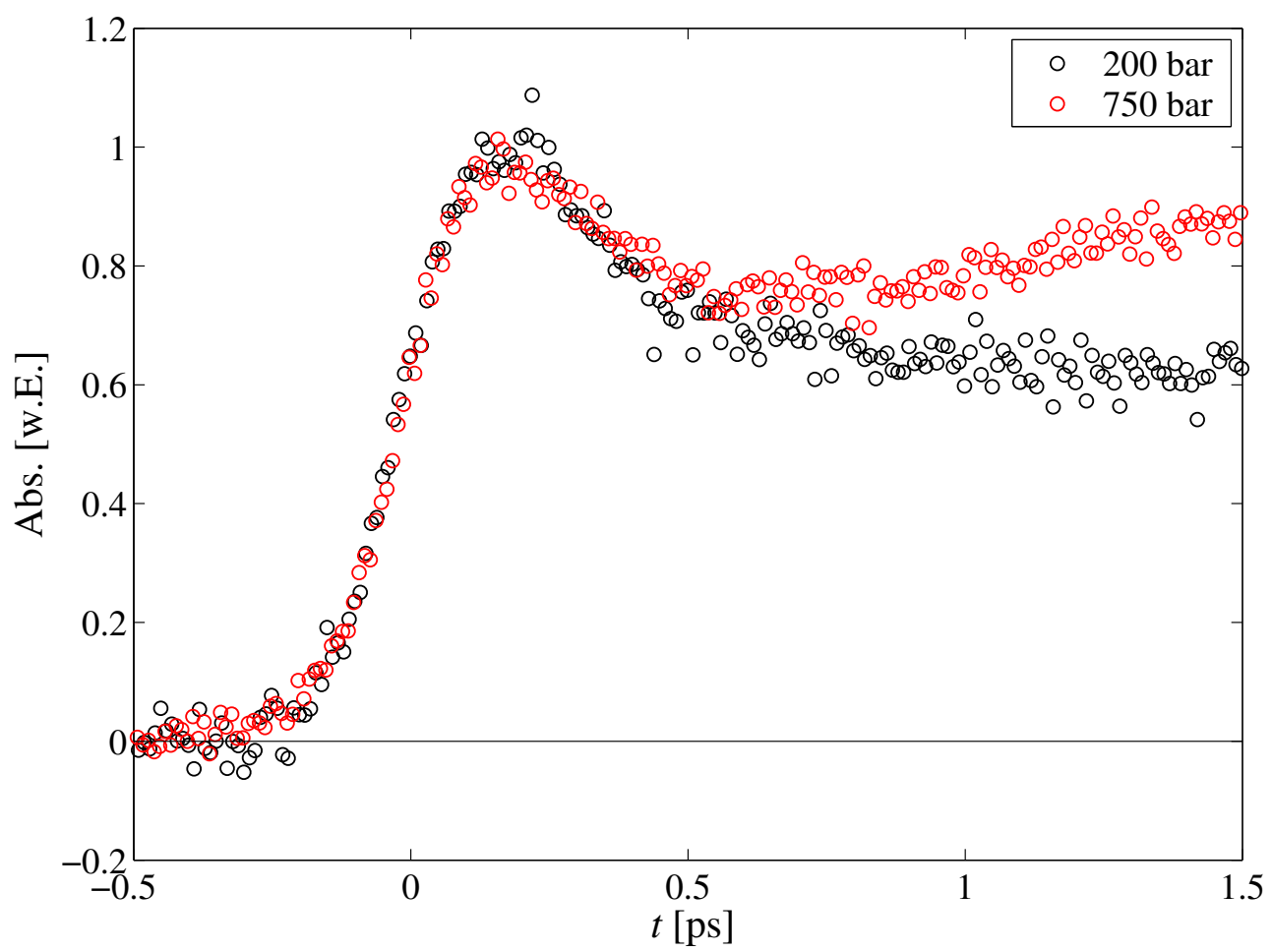

Abbildung 4.15: Absorptionszeitprofile zu frühen Verzögerungszeiten von $\mathrm{CH}_{2} \mathrm{I}_{2}$ in $\mathrm{CO}_{2}(200$ bzw. 750 bar, $35^{\circ} \mathrm{C}$ ) bei $300 \mathrm{~nm}$ Anregung und $405 \mathrm{~nm}$ Abfrage.

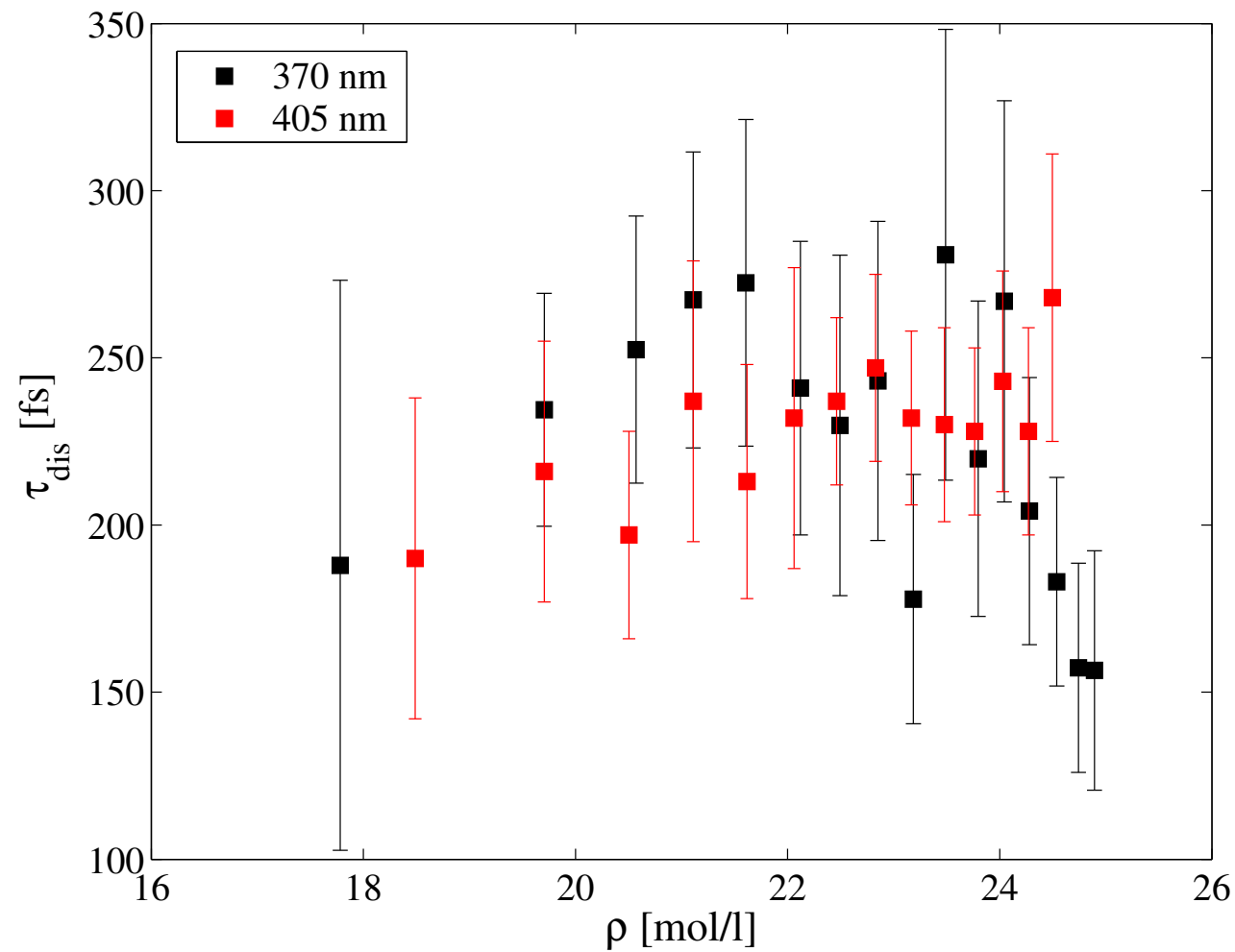

Abbildung 4.16: Auftragung von $\tau_{\text {dis }}$ gegen die Lösungsmitteldichte von $\mathrm{CO}_{2}\left(35^{\circ} \mathrm{C}\right)$ bei $300 \mathrm{~nm}$ Anregung und $405 \mathrm{~nm}$ Abfrage. 
Im Folgenden wird die Dichteabhängigkeit der Anstiegszeit $\tau$ sowie des schnellen Abfalls $\tau_{\text {dis }}$ beschrieben. Der langsame Anstieg wurde in allen Messungen bei einer Anregung von $304 \mathrm{~nm}$ mit einer einfach exponentiellen Funktion angepasst. Eine Auftragung der Geschwindigkeitskonstanten $k=1 / \tau$ gegen die Dichte bei verschiedenen Probewellenlängen ist in Abb.4.13 dargestellt. Die Fehlerbalken in dieser Auftragung entsprechen einem Konfidenzintervall von $66.7 \%$. Es ist ersichtlich, dass der langsame Anstieg von der Mitte der Absorptionsbande zu der roten Flanke hin, wie schon im voherigen Abschnitt beschrieben, schneller wird. Bei den einzelnen Wellenlängen ergibt sich eine nichtlinear mit der Dichte ansteigende Geschwindigkeitskonstante. Am quasi-isosbestischen Punkt bei der Probewellenlänge von $405 \mathrm{~nm}$ fällt die Lebensdauer von $\sim 23$ auf $\sim 8$ ps von den niedrigsten bis zu den höchsten erreichten Dichten ab. In der Auftragung 4.14 wurden die Messungen bei 435 und $405 \mathrm{~nm}$ Abfrage auf die Messung bei $370 \mathrm{~nm}$ skaliert. Der Kurvenverlauf ist bei allen drei Messreihen gleich, was bedeutet, dass die Dichteabhängigkeit von $k$ unabhängig von der Probewellenlänge ist.

Um zu klären, ob die schnelle abfallende Komponente mit der Lebensdauer $\tau_{\text {dis }}$ eine Dichteabhängigkeit aufweist, wurden zwei Dichteserien (bei 370 und $405 \mathrm{~nm}$ Abfrage) bis zu einer maximalen Verzögerung von 2 ps (hier nur bis 1.5 ps dargestellt) mit einer auf 10 fs verkürzten Schrittweite aufgenommen. Abb.4.15 zeigt zwei auf das Anfangsmaximum normierte Absorptionszeitprofile bei unterschiedlichen Dichten. Wie zu erkennen, können beide Prozesse, Abfall und anschließender Anstieg, nicht eindeutig voneinander getrennt werden. Die Lebensdauern $\tau_{\text {dis }}$ der beiden Dichteserien sind in Abb.4.16 zusammengefasst. Bei der Probewellenlänge von $405 \mathrm{~nm}$ ist ein ansteigender Trend sichtbar, bei der Abfragewellenlänge von $370 \mathrm{~nm}$ ist jedoch kein eindeutiger Trend zu erkennen. Die Verlangsamung mit steigenden Druck bei $405 \mathrm{~nm}$ Abfrage wird jedoch wahrscheinlich wie vorher beschrieben von dem langsamen Anstieg verursacht. Es konnte somit keine Dichteabhängigkeit der schnellen abfallenden Komponente aufgelöst werden.

\subsubsection{Lösungsmittelabhängigkeit}

Kohlendioxid ist auf Grund seiner guten Lösungseigenschaften das in dieser Arbeit am häufigsten eingesetzte Lösungsmittel. Um verschiedene Lösungsmitteleinflüsse zu untersuchen, wurden Messungen in drei weiteren Lösungsmitteln durchgeführt: Xenon, Ethan und Fluoroform.

Zur Untersuchung des Einflusses des Schwingungsenergietransfers wurden Xenon und Ethan verwendet. Xenon besitzt keine inneren Freiheitsgrade und kann daher Energie nur in den Translationsfreiheitsgraden aufnehmen. Im Gegensatz dazu besitzt Ethan 18 Schwingungsfreiheitsgrade sowie drei Rotationsfreiheitsgrade und kann somit wesentlich effektiver Schwingungsenergie aufnehmen. Um den Einfluss der Lösungsmittelpolarität auf die Dynamik zu untersuchen, wurde Fluoroform eingesetzt, weil es eine stark dichteabhängige Dielektrizitätskonstante besitzt [117,118]. Die vier in dieser Arbeit verwendeten Lösungsmittel weisen moderate kritische Temperaturen und Drücke auf (siehe Anhang A.1).

Die Spektren in Xenon wurden bei einer Anregungswellenlänge von $302 \mathrm{~nm}$ und einer reduzierten Temperatur $T_{\mathrm{r}}=1.06$, in Ethan bei $315 \mathrm{~nm}$ und $T_{\mathrm{r}}=1.02$ und in Fluoroform bei $315 \mathrm{~nm}$ und $T_{\mathrm{r}}=1.04$ durchgeführt. Die zeitaufgelösten Absorptionsspektren finden sich im Anhang A.2. Dabei zeigt sich in Xenon eine deutlich langsamere Dynamik als bei den 


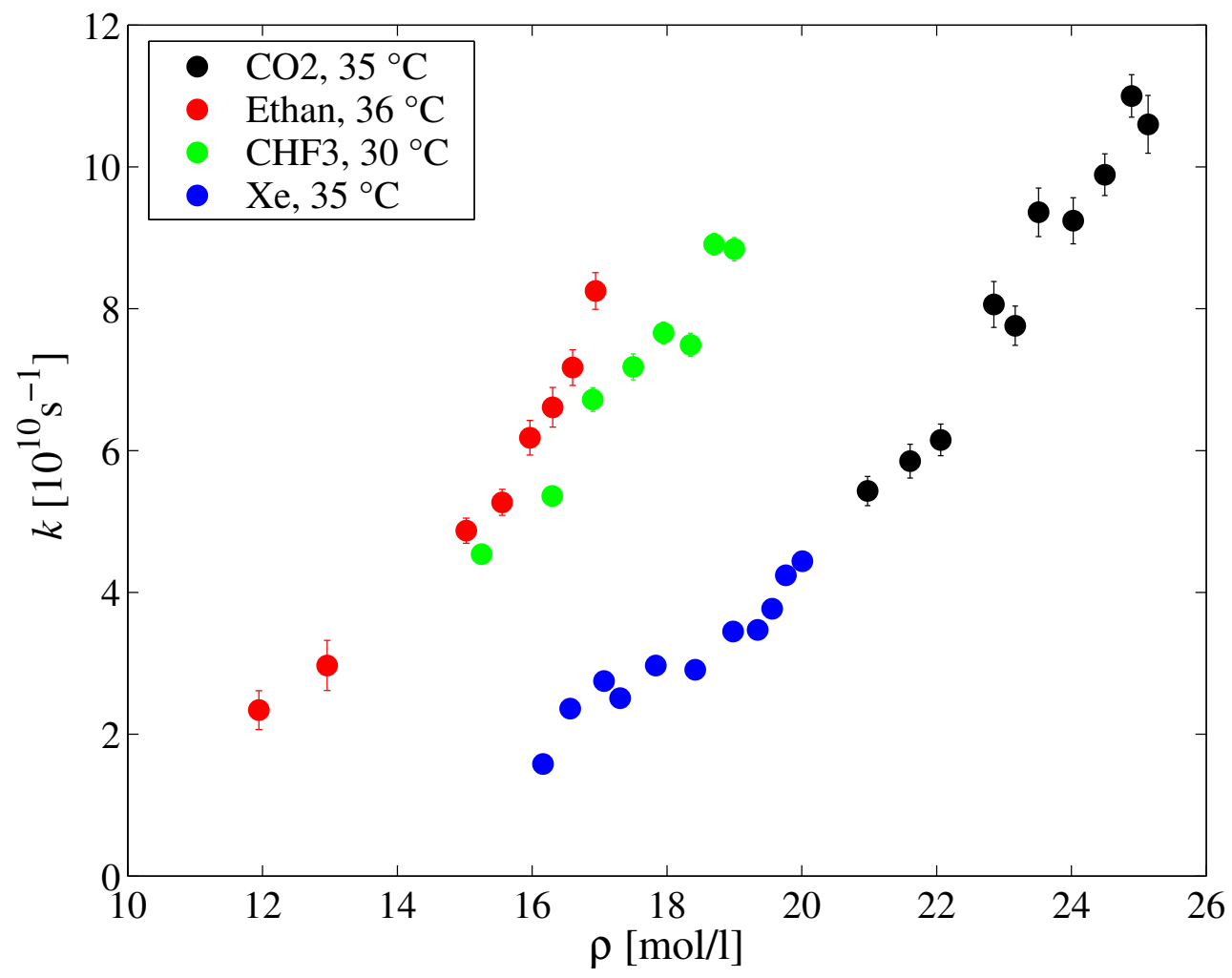

Abbildung 4.17: Auftragung von $k$ gegen die Lösungsmitteldichte von $\mathrm{CO}_{2}, \mathrm{C}_{2} \mathrm{H}_{6}, \mathrm{CHF}_{3}$ und Xe bei $405 \mathrm{~nm}$ Abfrage.

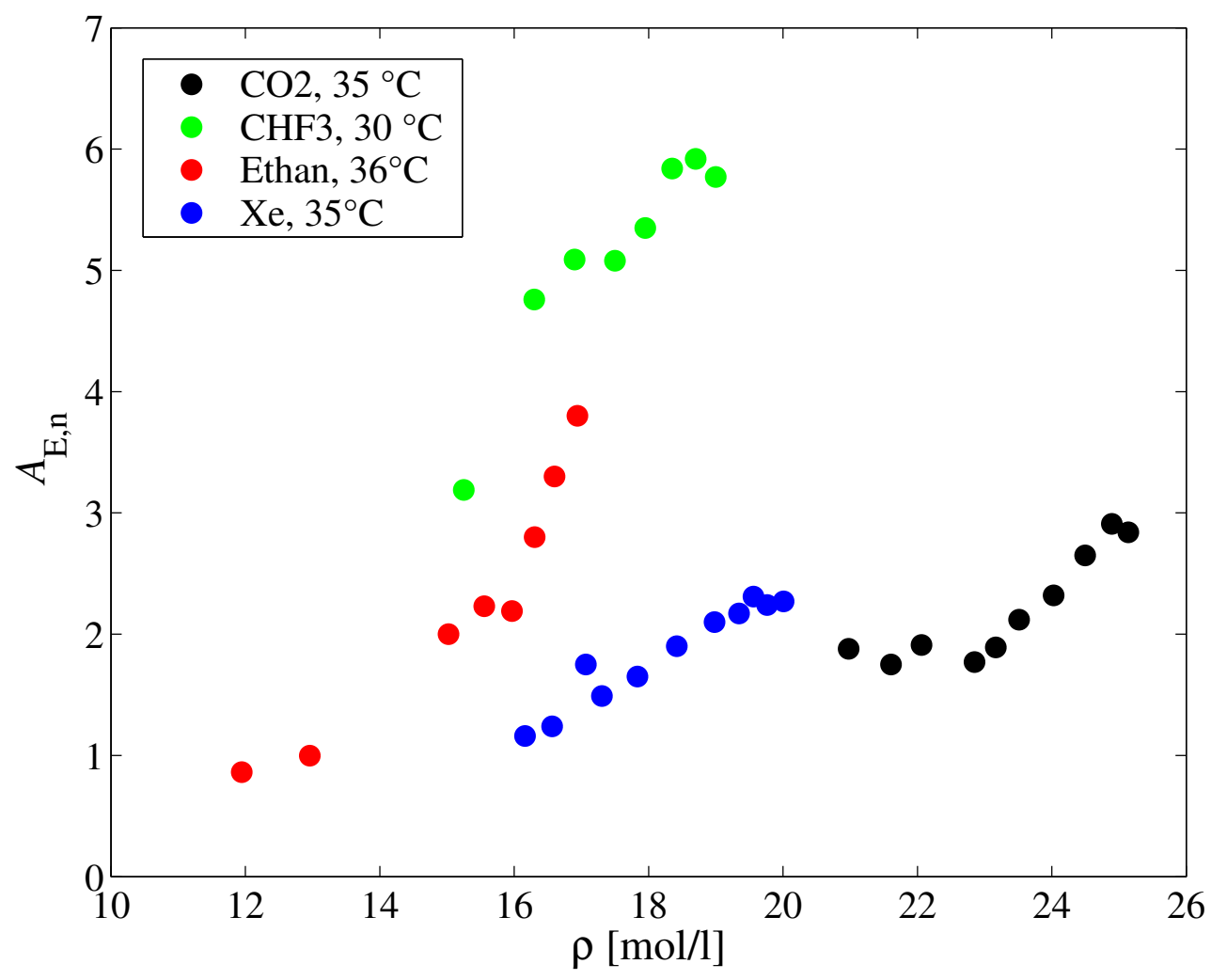

Abbildung 4.18: Auftragung der normierten Endamplitude $A_{\mathrm{E}, \mathrm{n}}$ gegen die Lösungsmittel40 dichte von $\mathrm{CO}_{2}, \mathrm{C}_{2} \mathrm{H}_{6}, \mathrm{CHF}_{3}$ und Xe bei $405 \mathrm{~nm}$ Abfrage. 


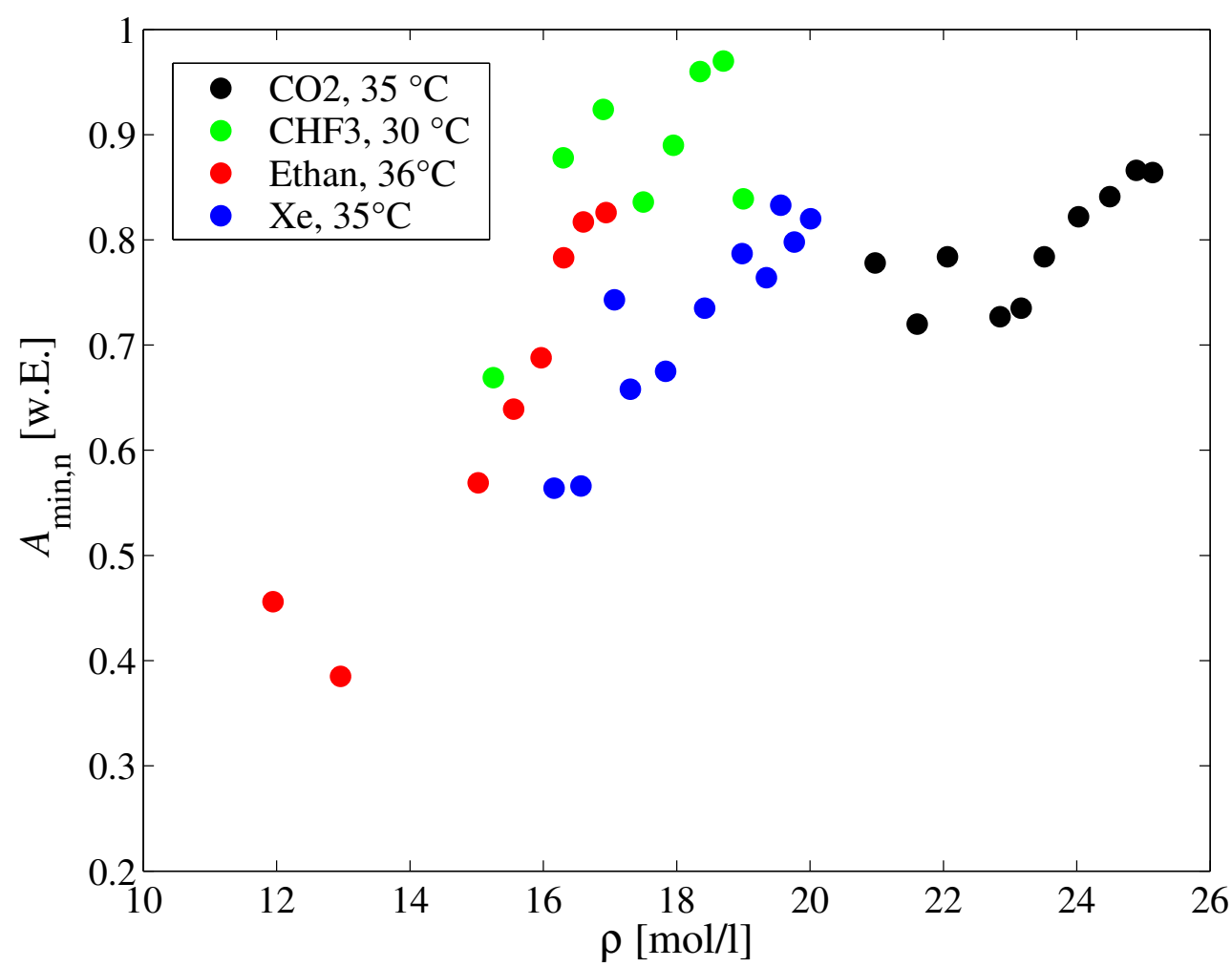

Abbildung 4.19: Auftragung der normierten Minima $A_{\text {min,n }}$ gegen Lösungsmitteldichte von $\mathrm{CO}_{2}, \mathrm{C}_{2} \mathrm{H}_{6}, \mathrm{CHF}_{3}$ und Xe bei $405 \mathrm{~nm}$ Abfrage.

anderen verwendeten Lösungsmitteln (siehe Abb.A.1). Der quasi-isosbestische Punkt (siehe Abb.A.2 liegt wie in $\mathrm{CO}_{2}$ bei $405 \mathrm{~nm}$. Das zeitaufgelöste Absorptionsspektrum in Ethan (siehe Abb.A.3) zeigt eine ähnliche Form wie in $\mathrm{CO}_{2}$. Es konnte ebenfalls bei $405 \mathrm{~nm}$ ein quasi-isosbestischer Punkt gefunden werden (siehe Abb. A.4). Das Spektrum in dem polaren Lösungsmittel $\mathrm{CHF}_{3}$ (siehe Abb. A.5) ist gegenüber den Spektren in den anderen Lösungsmitteln geringfügig rotverschoben, was an dem quasi-isosbestischen Punkt bei $410 \mathrm{~nm}$ erkennbar ist (siehe Abb. A.6). Diese solvatochromatische Rotverschiebung mit zunehmender Lösungsmittelpolarität wurde ebenfalls von Tarnovsky et al. beobachtet [63]. Im Gegensatz zu dem $\mathrm{CO}_{2}$-Spektrum wurden diese Spektren nur bis zu einer Probewellenlänge von $355 \mathrm{~nm}$ aufgenommen. Trotzdem zeigt sich in allen Spektren die dem $\mathrm{CH}_{2}$ I-Radikal zugeordnete Struktur an der blauen Flanke. Typische Absorptionszeitprofile sind in Anhang A.4 abgebildet.

Weil, wie im folgenden Abschnitt dargestellt, die Lebensdauer des langsamen Anstiegs stark von der Anregungswellenlänge abhängt, wurde für den direkten Vergleich der verschiedenen Lösungsmittel eine Messreihe in $\mathrm{CO}_{2}$, Ethan und $\mathrm{CHF}_{3}$ bei der gleichen reduzierten Temperatur $\left(T_{\mathrm{r}}=1.01\right)$ und bei $300 \mathrm{~nm}$ Anregung sowie $405 \mathrm{~nm}$ Abfrage durchgeführt. Die Messungen in Xe hingegen wurden bei $T_{\mathrm{r}}=1.06$ und $302 \mathrm{~nm}$ Anregung durchgeführt. Die Geschwindigkeitskonstanten $k$ der verschiedenen Lösungsmittel werden in Abb.4.17 verglichen. Die Daten für die Umrechnung von der Druck- in eine Dichteskala für Xenon und Ethan wurden [116] entnommen während die Umrechnung für Fluoroform nach einer em- 
pirischen Formel [92, 119] erfolgte. Auch in dieser Auftragung zeigt sich, dass die Dynamik in überkritischem Xenon deutlich langsamer als in den anderen verwendeten Lösungsmitteln ausfällt. In Abb.4.18 sind die normierten Endamplituden $A_{\mathrm{E}, \mathrm{n}}$ in Abhängigkeit von der Lösungsmitteldichte aufgetragen. Abb.4.19 zeigt das entsprechende Bild für die normierten Minima $A_{\text {min,n }}$.

\subsubsection{Anregungswellenlängenabhängigkeit}

Es wurde der Einfluss der Anregungswellenlänge auf die Dynamik der Photoisomerisierung untersucht. Bei kürzeren Anregungswellenlängen steigen die innere Energie des $\mathrm{CH}_{2} \mathrm{I}-$ Radikals, die kinetische Energie der Photofragmente und die Quantenausbeute an angeregten Iodatomen an (siehe Abschn.2.2.1). Als Lösungsmittel wurde Kohlendioxid gewählt. Zusätzlich zu den in Abschn.4.2.2 abgebildeten Messungen bei 304 nm wurden dichteabhängige Absorptionszeitprofile bei 263, 272 und $340 \mathrm{~nm}$ Anregung bei jeweils $405 \mathrm{~nm}$ Abfrage aufgenommen. Bei der Anregung mit der geringsten Überschussenergie (340 nm) wurde der langsame Anstieg mit einer zweifach exponentiellen Funktion angepasst, bei den übrigen mit einer einfach exponentiellen. Abb. 4.20 zeigt eine solche Messung mit zweifach exponentiellem Anstieg. In der Vergrößerung der Abbildung ist ersichtlich, dass die Zeitauflösung bei dieser Pumpwellenlänge wesentlich geringer als bei den anderen Pumpwellenlängen ausfällt $(\sim 0.5 \mathrm{ps})$. Verursacht wurde dies wahrscheinlich durch zwei zeitlich versetzte Pumppulse. Dies hat zur Konsequenz, dass der schnelle Abfall nicht aufgelöst werden konnte; die Absorptionszeitprofile wurden lediglich mit einem zweifach exponentiellen Anstieg angepasst. Die langsame Komponente besitzt dabei eine Lebensdauer von $\sim 60$ ps, die schnelle $5-8$ ps. In Abb.4.21 ist die Geschwindigkeitskonstante der langsamen Komponente $k_{2}$ sowie ihre relative Amplitude dargestellt. Aus dieser Abbildung geht hervor, dass sich für die Geschwindigkeitskonstante keine Dichteabhängigkeit auflösen lässt, jedoch sinkt ihr relativer Anteil mit zunehmenden Druck. Um zu klären, ob sich durch Variation der Anregungsenergie das transiente Spektrum verschiebt, wurde ein zeitaufgelöstes Spektrum bei $340 \mathrm{~nm}$ Anregung (siehe Abb.4.22) aufgenommen. Es zeigt eine ähnliche spektrale Evolution und einen quasi-isosbestischen Punkt wie das Spektrum bei $304 \mathrm{~nm}$ Anregung (vergleiche Abb.4.5).

Abb. 4.23 zeigt die Geschwindigkeitskonstante $k$ bei allen vier Anregungswellenlängen. Für die Dichteserie bei $340 \mathrm{~nm}$ Anregung wurde sowohl die Geschwindigkeitskonstante für die schnelle Komponente als auch das amplitudengewichtete Mittel aus beiden Komponenten dargestellt. Es zeigt sich, dass mit zunehmender Überschussenergie die Dynamik deutlich langsamer wird, die Kurvenform jedoch bei allen drei Messreihen ähnlich ist. Die absoluten Endamplituden $A_{\mathrm{E}}$ konnten wegen unterschiedlicher Pumpenergie nicht miteinander verglichen werden. Durch verschiedene Anregungswellenlängen wird in verschiedene elektronische Zustände angeregt, so dass die Übergangsmomente für den $S_{\mathrm{n}} \longleftarrow S_{1}$-Übergang ebenfalls verschieden sind. Die Anfangsabsorption ist dementsprechend eine Funktion der Anregungswellenlänge und die normierten Endamplituden eignen sich somit ebenfalls nicht zum Vergleich. Als Konsequenz ist eine Aussage über die Anregungswellenlängenabhängigkeit der Quantenausbeute $\Phi_{\text {iso }}$ nicht möglich. 

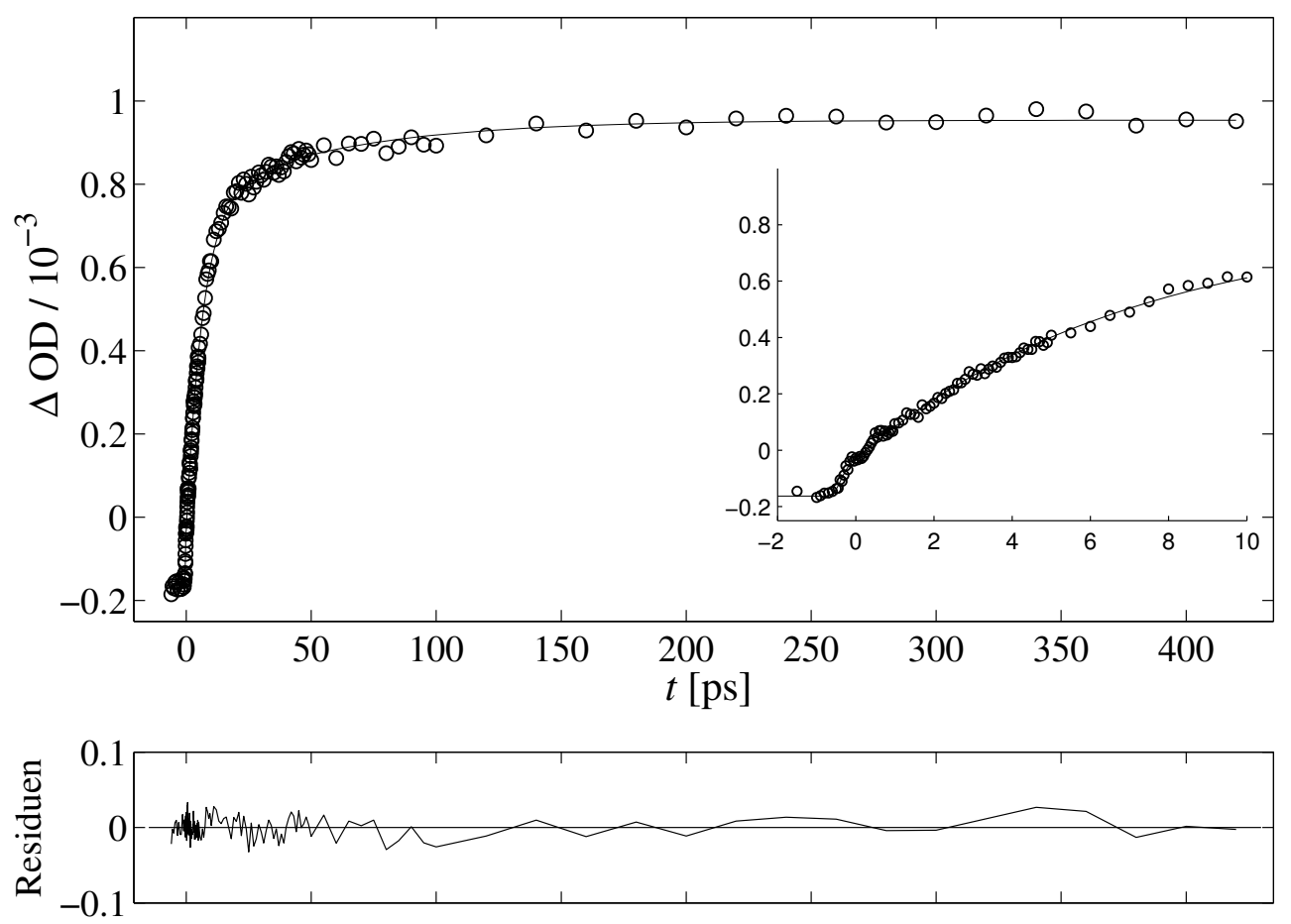

Abbildung 4.20: Absorptionszeitprofil von $\mathrm{CH}_{2} \mathrm{I}_{2}$ in $\mathrm{CO}_{2}\left(404\right.$ bar, $\left.35^{\circ} \mathrm{C}\right)$ bei $340 \mathrm{~nm}$ Anregung und $405 \mathrm{~nm}$ Abfrage.

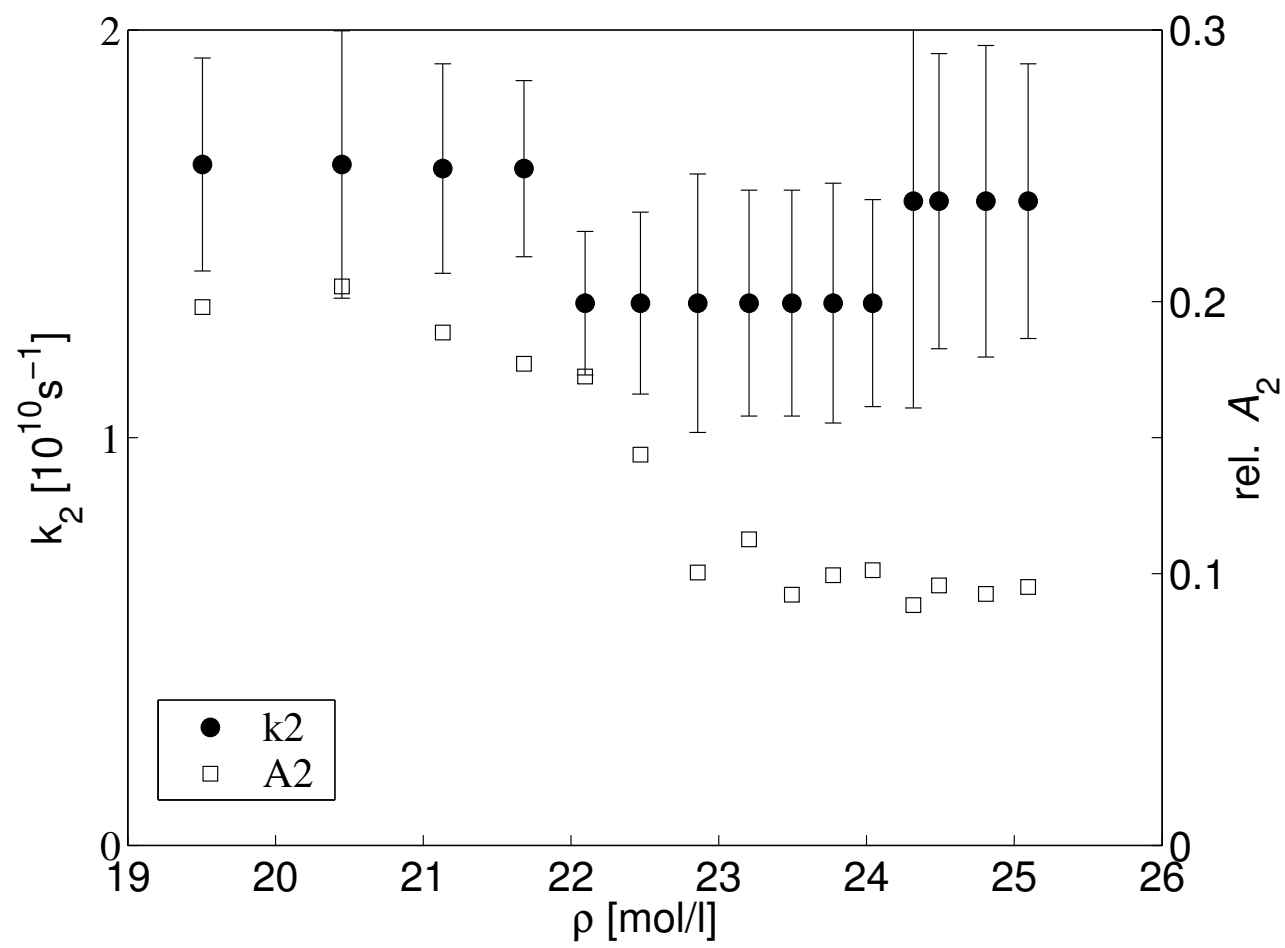

Abbildung 4.21: Auftragung von $k_{2}$ und der relativen Amplitude $A_{2}$ gegen die Lösungsmitteldichte von $\mathrm{CO}_{2}\left(35^{\circ} \mathrm{C}\right)$ bei $340 \mathrm{~nm}$ Anregung und $405 \mathrm{~nm}$ Abfrage. 


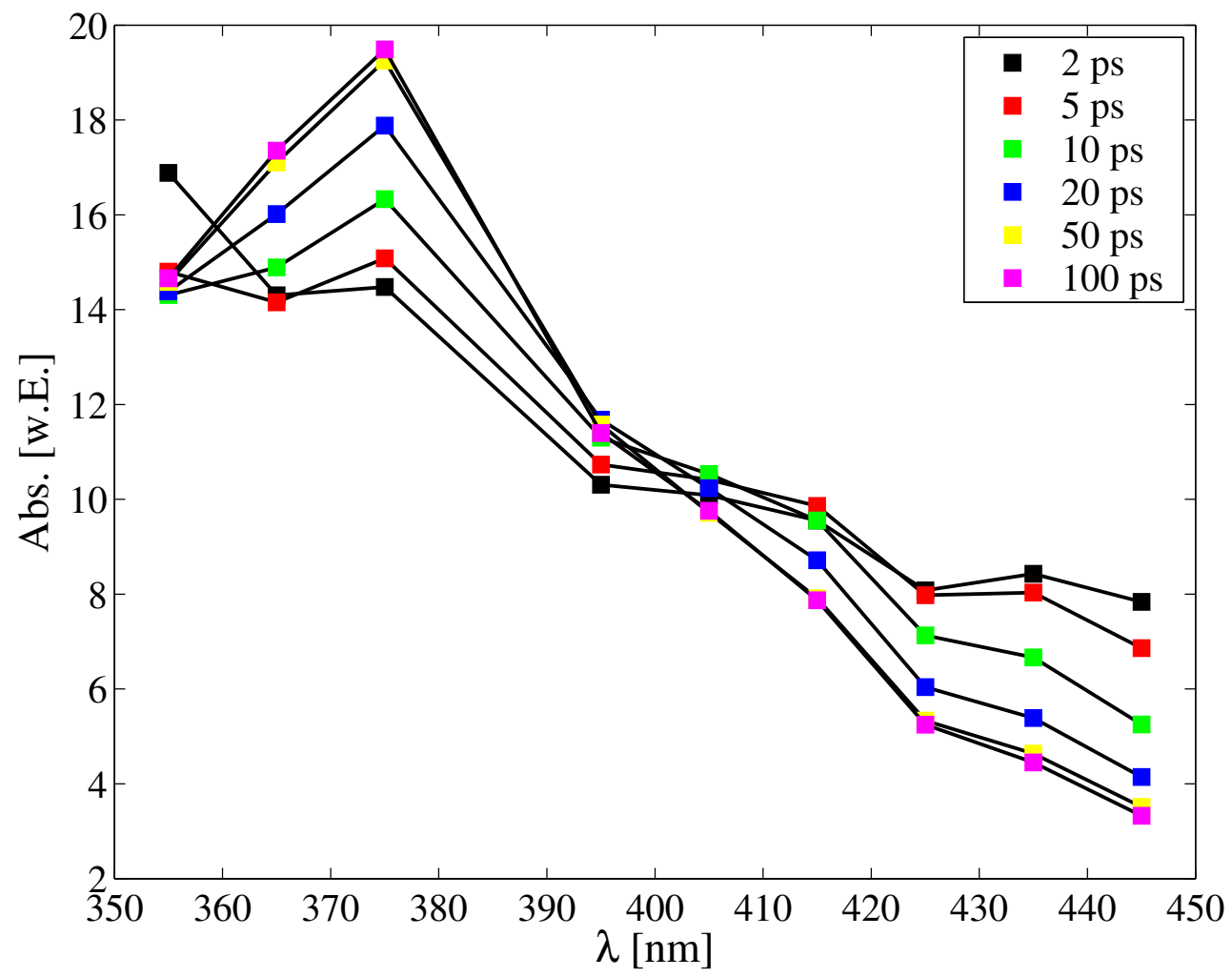

Abbildung 4.22: Rekonstruiertes und normiertes zeitaufgelöstes Spektrum von $\mathrm{CH}_{2} \mathrm{I}_{2}$ in $\mathrm{CO}_{2}$ (400 bar, $35^{\circ} \mathrm{C}$ ) bei $340 \mathrm{~nm}$ Anregung.

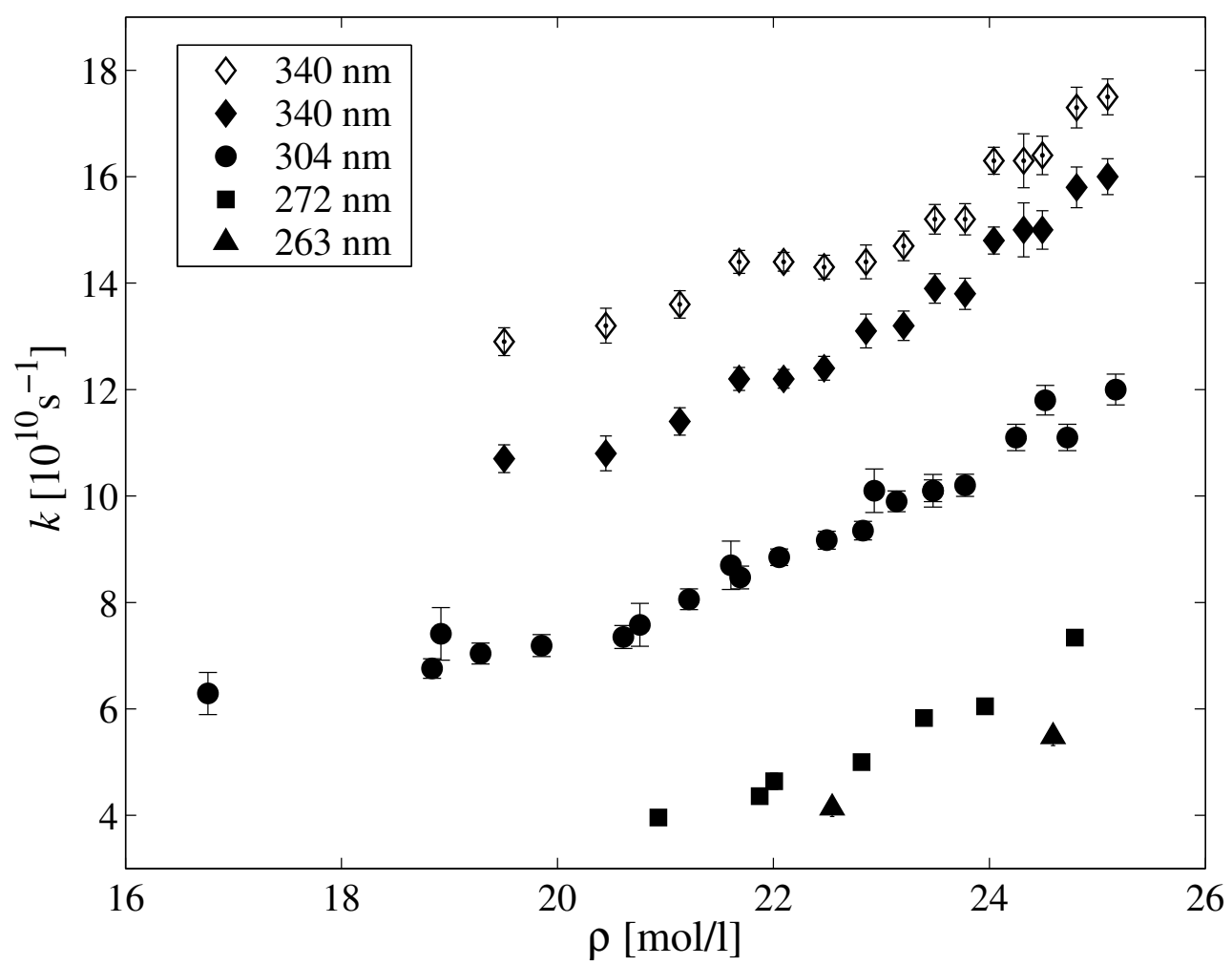

Abbildung 4.23: Dichteabhängigkeit von $k$ in $\mathrm{CO}_{2}$ bei verschiedenen Anregungswellenlängen $\left(405 \mathrm{~nm}\right.$ Probe, $\left.35^{\circ} \mathrm{C}\right)$. Die offenen Symbole bei $340 \mathrm{~nm}$ stellen die schnelle Komponente dar, die geschlossenen die gemittelten aus schneller und langsamer Komponente. 


\subsection{Zeitaufgelöste Absorptionsmessungen an $\mathrm{CH}_{2} \mathrm{BrI}$}

Die zweite in dieser Arbeit untersuchte Dihalogenverbindung ist das Bromiodmethan $\mathrm{CH}_{2} \mathrm{BrI}$. Die Intensität der Absorption des $\mathrm{CH}_{2} \mathrm{Br}-\mathrm{I}$-Isomers ist vergleichbar mit der des $\mathrm{CH}_{2} \mathrm{I}-\mathrm{I}$ (siehe Abb.2.7), jedoch ist die Quantenausbeute $\Phi_{\text {iso }}$ um ein Drittel geringer (vergl. Abschn.2.2.2). Mithin ist eine um ein Drittel geringere Signalintensität zu erwarten. Aufgrund dieses geringeren Signal-Rausch-Verhältnisses konnte nur eine Dichteserie bei einer Abfragewellenlänge $400 \mathrm{~nm}$ erhalten werden. Abb.4.24 zeigt ein Absorptionszeitprofil von Bromiodmethan in überkritischem Kohlendioxid. Das Signal zeigt die gleichen drei Komponenten wie die Transienten des Diiodmethans: schneller Anstieg innerhalb der Zeitauflösung, schneller Abfall sowie langsamer Anstieg auf einer Picosekundenzeitskala. Die auf das Anfangsmaximum normierten Endamplituden sind in Abb.4.25, die Geschwindigkeitskonstanten in Abb.4.26 dargestellt. Die Lebensdauern des langsamen Anstiegs liegen zwischen $\sim 13$ und $\sim 24$ ps. Ebenso wie bei den Messungen des Diiodmethans ergibt sich in beiden Auftragungen ein nichtlinear ansteigender funktioneller Zusammenhang der beiden Messgrößen mit der Dichte.
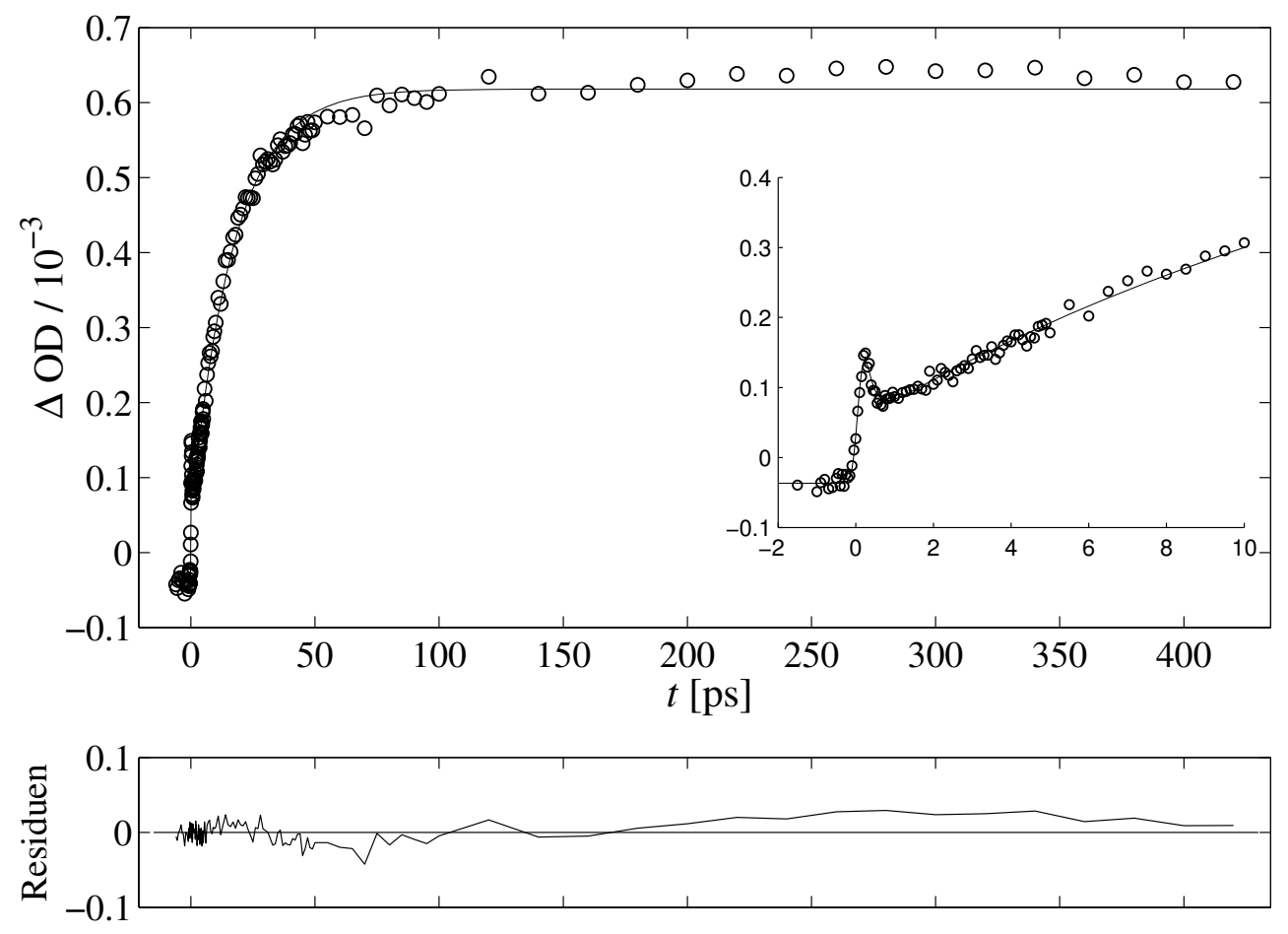

Abbildung 4.24: Absorptionszeitprofil von $\mathrm{CH}_{2} \mathrm{BrI}$ in $\mathrm{CO}_{2}\left(551\right.$ bar, $\left.35^{\circ} \mathrm{C}\right)$ bei $288 \mathrm{~nm}$ Anregung und $400 \mathrm{~nm}$ Abfrage. 


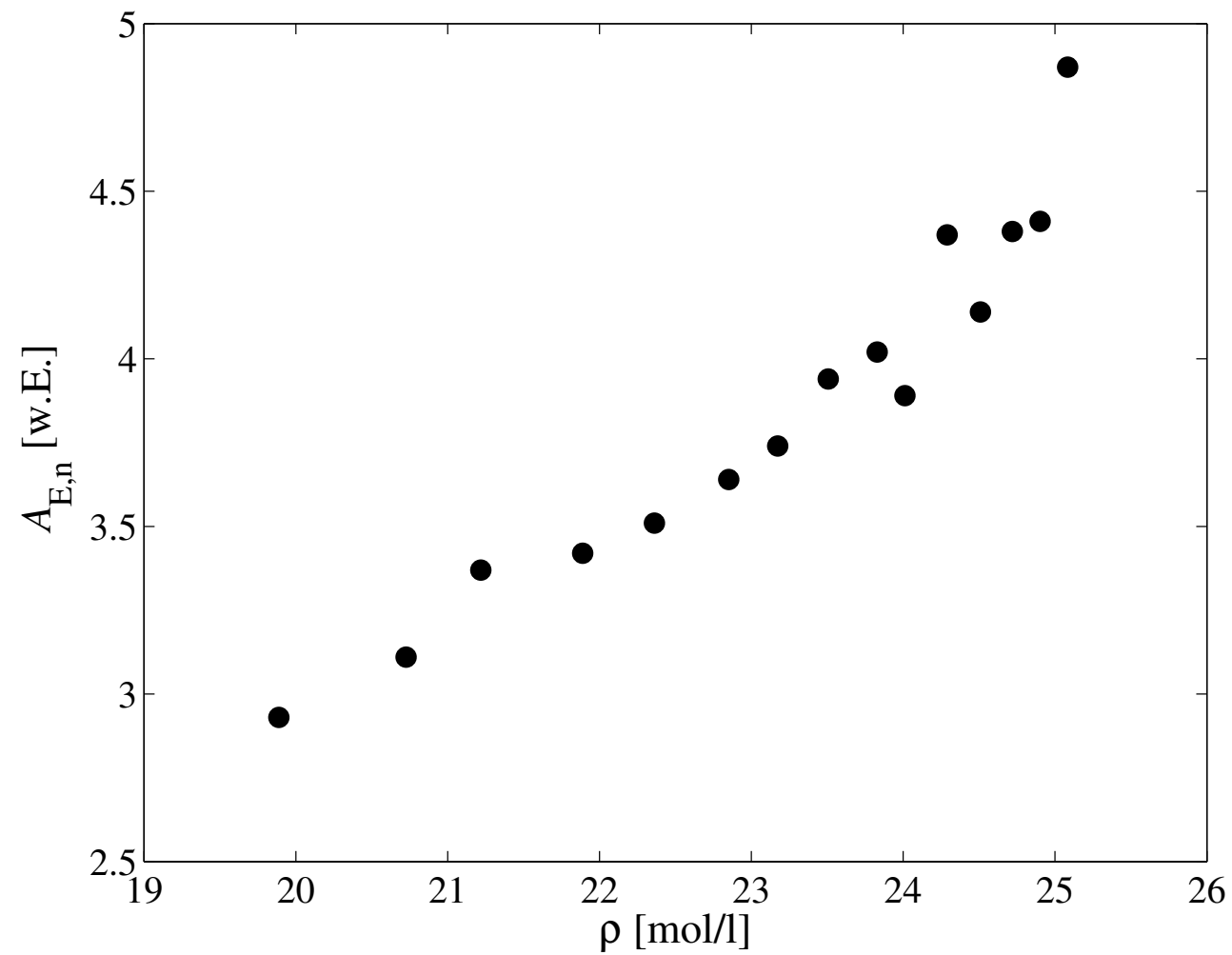

Abbildung 4.25: Auftragung der normierten Endamplitude $A_{\mathrm{E}, \mathrm{n}}$ gegen die Lösungsmitteldichte von $\mathrm{CH}_{2} \mathrm{BrI}$ in $\mathrm{CO}_{2}$ bei $288 \mathrm{~nm}$ Anregung und $400 \mathrm{~nm}$ Abfrage.

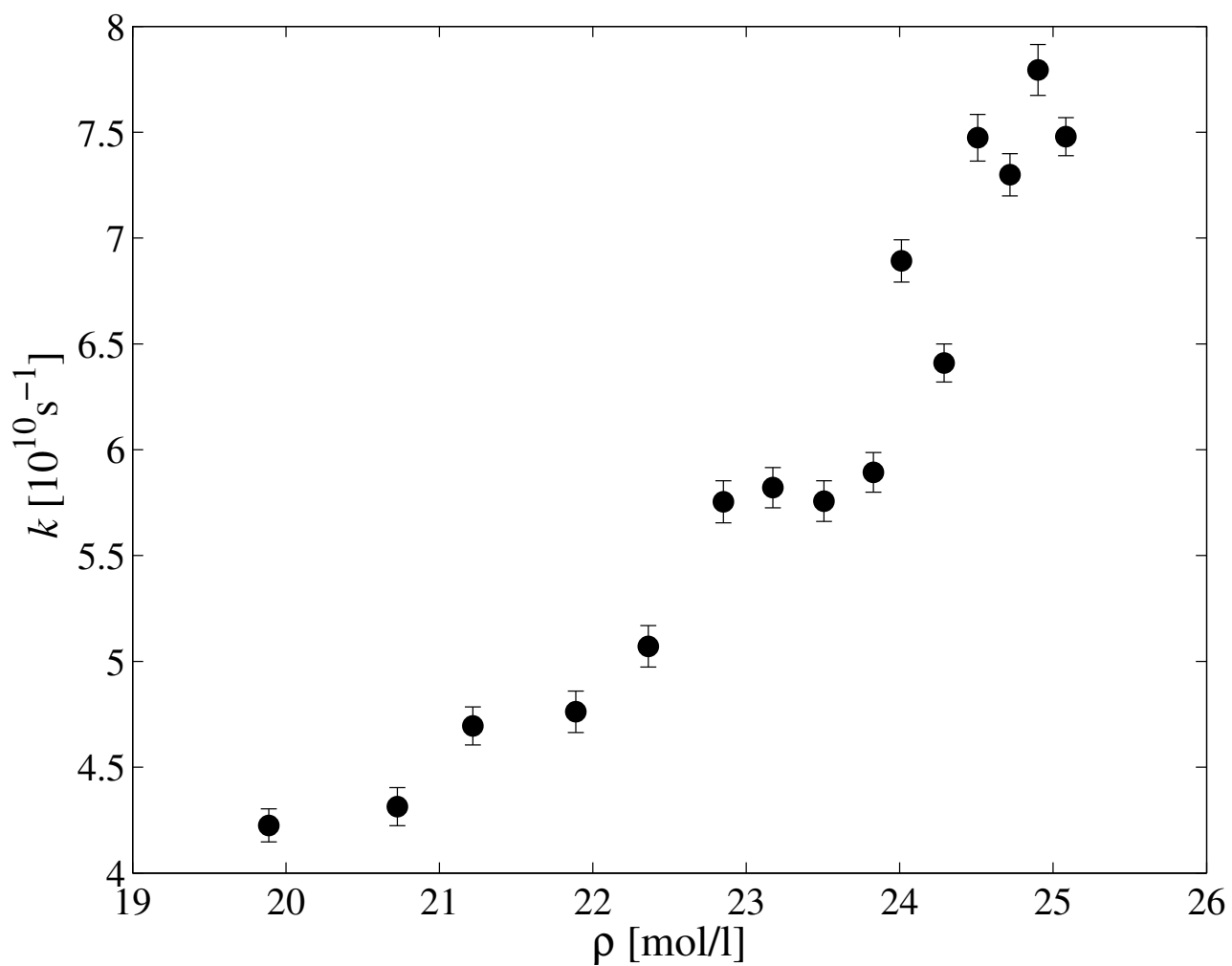

Abbildung 4.26: Auftragung von $k$ von $\mathrm{CH}_{2} \mathrm{BrI}$ gegen die Lösungsmitteldichte von $\mathrm{CO}_{2}$ bei $288 \mathrm{~nm}$ Anregung und $400 \mathrm{~nm}$ Abfrage. 


\subsection{Zeitaufgelöste Anisotropiemessungen}

Es wurden dichteabhängige Absorptionszeitprofile von Diiodmethan in Kohlendioxid bei paralleler und senkrechter Polarisation von Pump- und Probestrahl aufgenommen und zu langen Verzögerungszeiten aufeinander abgeglichen. Dabei zeigt sich zu Beginn der Dynamik bei paralleler Polarisation eine höhere Intensität als bei senkrechter (siehe Abb.4.27). Die Anisotropie $r$ (siehe Gl.3.4) wurde aus diesen aufeinander abgeglichenen Messungen berechnet und ebenfalls in Abb.4.27 dargestellt. Bei den höheren Dichten wurde die Anisotropie direkt nach dem schnellen Abfall, bei den niedrigeren Dichten ab dem Maximum angepasst. Es ergab sich in allen Messungen mit einer Anfangsanisotropie $r_{0}$ von $0.20 \pm 0.05$ einen deutlich kleineren Wert als 0.4, was bedeutet, dass die Übergangsdipolmomente von Anregung und Probe deutlich verschieden orientiert sind (vergl. Abschn. 3.4).

Die Anisotropie $r(t)$ wurde mit einer einfach abfallenden ungefalteten Exponentialfunktion angepasst:

$$
r(t)=r_{0} \exp \left(\frac{-\left(t-t_{0}\right)}{\tau_{\text {rot }}}\right)
$$

Der Anpassungsparameter ist die Lebensdauer der Orientierungsrelaxation $\tau_{\text {rot }}$. Die Orientierungsrelaxation aller durchgeführten Anisotropiemessungen ist in Abb.4.28 dargestellt. Es lässt sich bei den beiden verwendeten Probewellenlängen (370 und $405 \mathrm{~nm}$ ) keinen Unterschied erkennen. Beide Messreihen zeigen sich deutliche Verlangsamung der Orientierungsrelaxation mit zunehmender Dichte.

Desweiteren wurde eine Anisotropiemessung von Bromiodmethan in $\mathrm{CO}_{2}$ aufgenommen (siehe Abb.4.29). Die Messung weist ein geringeres Signal-Rausch-Verhältnis auf als die entsprechenden $\mathrm{CH}_{2} \mathrm{I}_{2}$-Messungen. Es ist jedoch eindeutig ersichtlich, dass die Anfangsanisotropie (am Maximum der Absorptionszeitprofile) des $\mathrm{CH}_{2} \mathrm{BrI}$ deutlich geringer ausfällt. Die transiente Absorption zeigt praktisch keine Anisotropie; es kann als hier keine Orientierungsrelaxation $\tau_{\text {rot }}$ angegeben werden. 


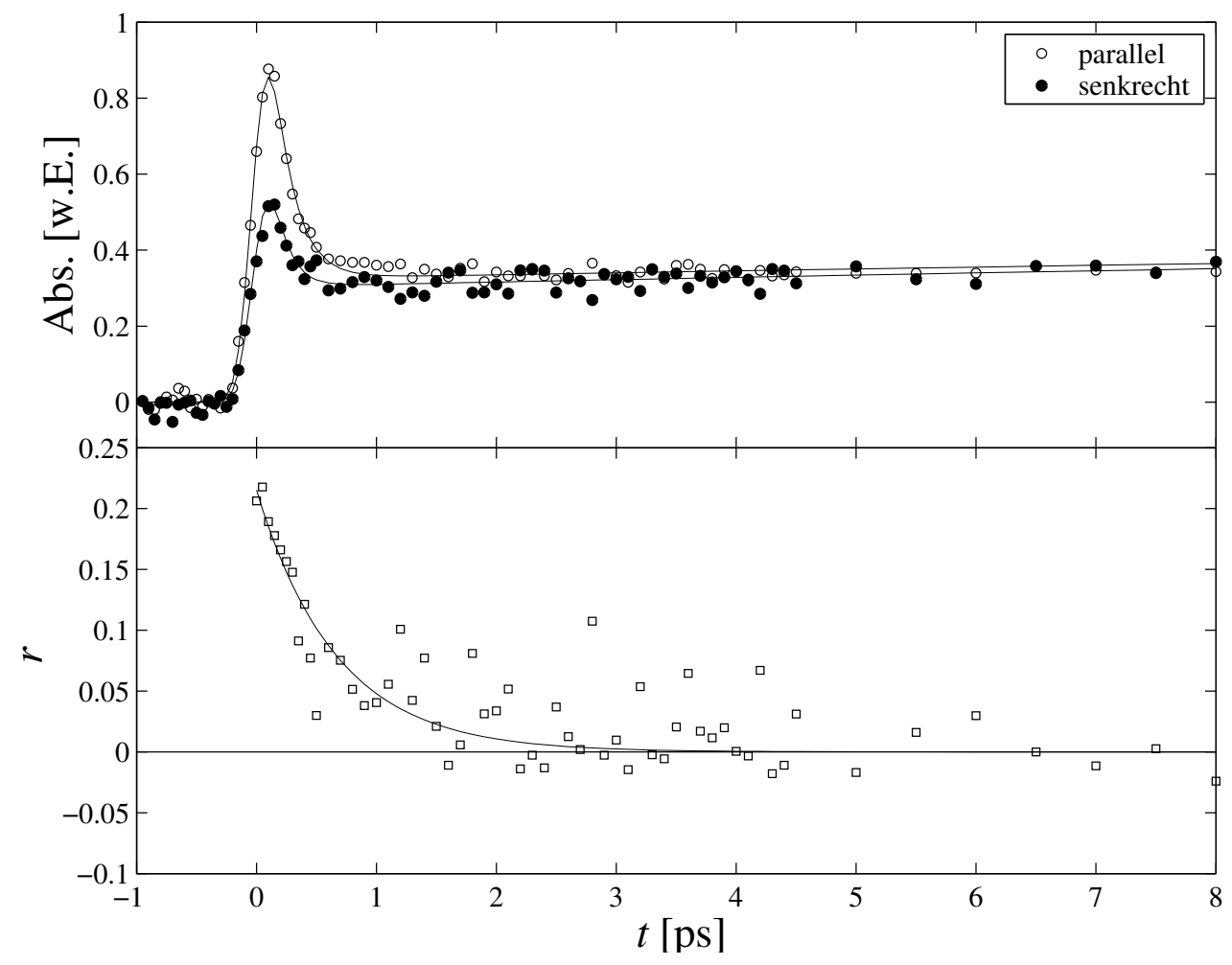

Abbildung 4.27: Oben: Absorptionszeitprofile von $\mathrm{CH}_{2} \mathrm{I}_{2}\left(\mathrm{CO}_{2}, 50^{\circ} \mathrm{C}, 103\right.$ bar $)$ bei $302 \mathrm{~nm}$ Anregung und $370 \mathrm{~nm}$ Abfrage bei paralleler und senkrechter Polarisation von Pump- und Probestrahl. Unten: Darstellung der Anisotropie $r(t)$.

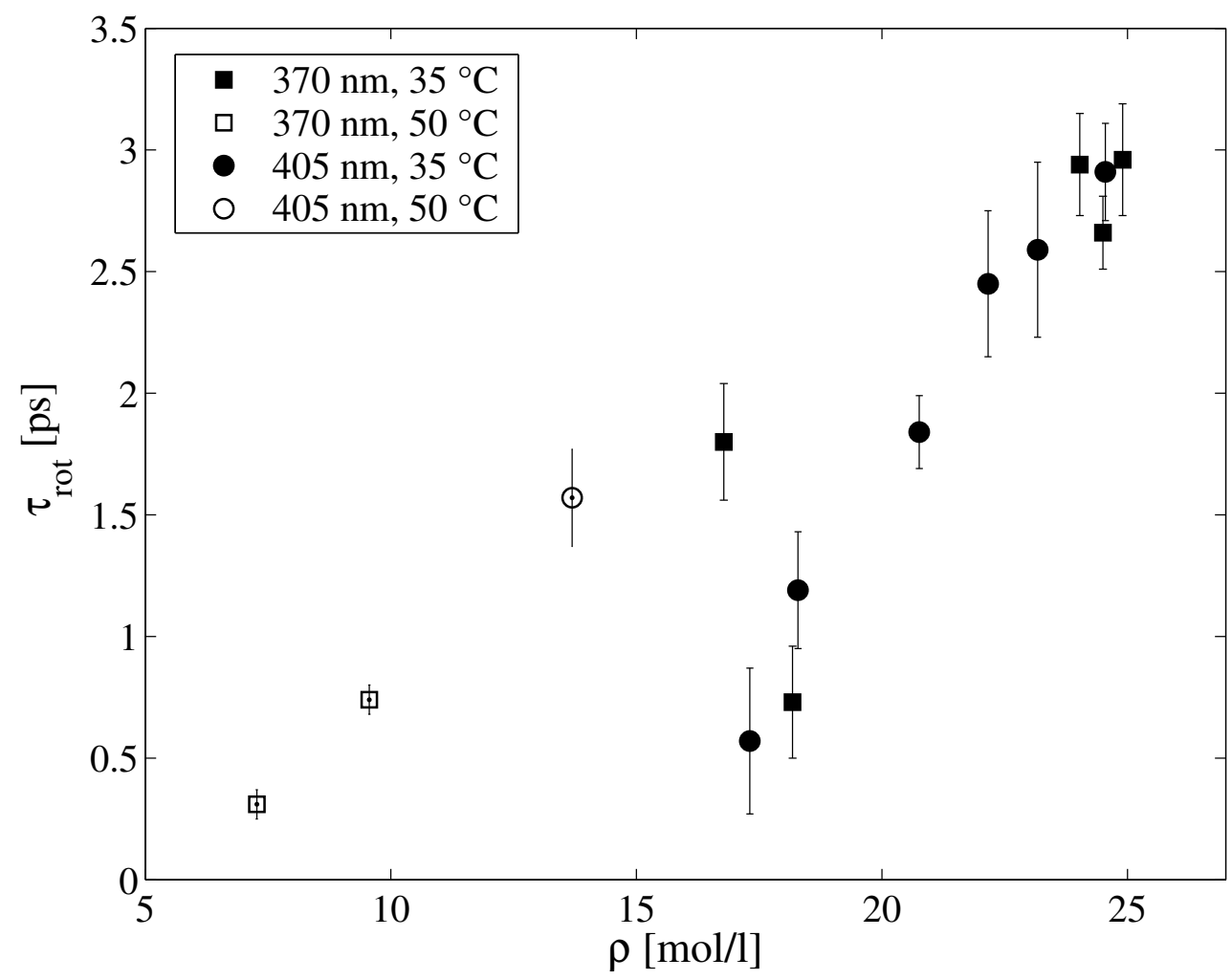

Abbildung 4.28: Auftragung der Orientierungsrelaxation $\tau_{\text {rot }}$ von $\mathrm{CH}_{2} \mathrm{I}_{2}$ gegen die Lösungsmitteldichte von $\mathrm{CO}_{2}$ bei 370 und $405 \mathrm{~nm}$ Probe. 


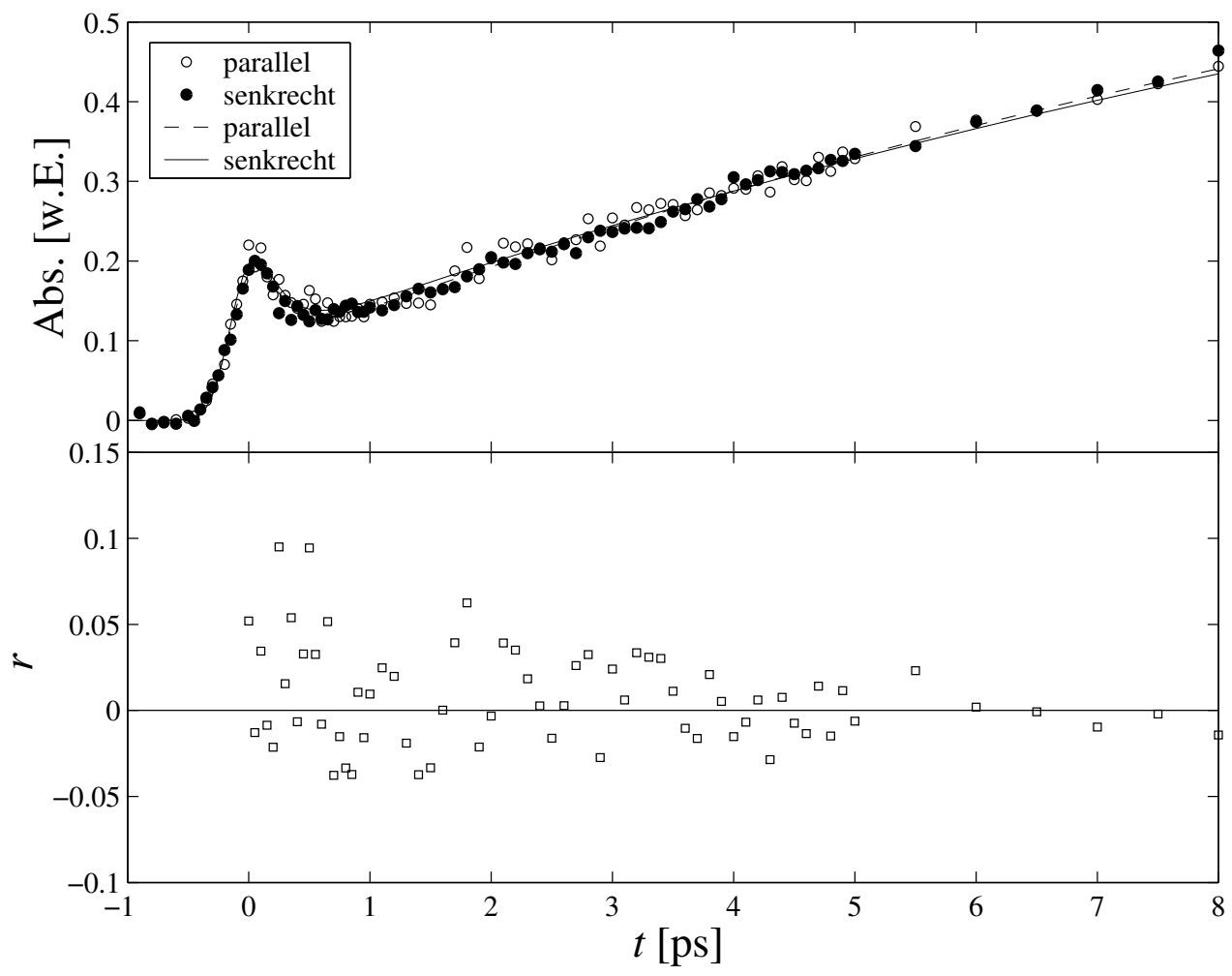

Abbildung 4.29: Oben: Absorptionszeitprofile von $\mathrm{CH}_{2} \mathrm{BrI}\left(\mathrm{CO}_{2}, 35^{\circ} \mathrm{C}, 928\right.$ bar $)$ bei $288 \mathrm{~nm}$ Anregung und $400 \mathrm{~nm}$ Abfrage bei paralleler und senkrechter Polarisation von Pump- und Probestrahl. Unten: Darstellung der Anisotropie $r$. 



\section{Molekulardynamische Simulationen}

In dieser Arbeit wurden klassische molekulardynamische Simulationen [86] zur Modellierung der Solvatstruktur der verschiedenen verwendeten Lösungsmittel um das Diiodmethan durchgeführt. Dazu wurden dichteabhängige Paar-Korrelationsfunktionen berechnet. Aus diesen Verteilungsfunktionen wurde die mittlere lokale Dichte entnommen, um mögliche Beiträge einer lokalen Dichtevergrößerung (siehe Abschn.2.4) auf die Photoisomerisierung zu untersuchen.

\subsection{Verwendete Potentiale}

Die Potentialparameter für das Diiodmethan und die verwendeten Lösungsmittel Kohlendioxid, Ethan, Fluoroform und Xenon wurden aus den Arbeiten von Stoll, Vrabec und Hasse [120, 121, 122, 123] entnommen. Die Parameter wurden dabei den Dampfdruckkurven der reinen Stoffe im Temperaturbereich $0.55 T_{\mathrm{c}}<T<0.95 T_{\mathrm{c}}$ angepasst. Obwohl die Potentiale dementsprechend nicht zur Beschreibung der überkritischen Phase entwickelt wurden, wurden sie in dieser Arbeit verwendet, weil so ein konsistenter Parametersatz für das Diiodmethan und die verwendeten Lösungsmittel vorliegt.

Im Folgenden werden die Potentiale beschrieben: Für die Diiodmethan-Diiodmethan Wechselwirkung wird ein Lennard-Jones Potential zusammen mit einer Punktdipol Wechselwirkung verwendet. Das Lennard-Jones Potential $u_{\mathrm{LJ}}$ hat die Form

$$
u_{\mathrm{LJ}}=4 \epsilon\left[\left(\frac{\sigma}{r}\right)^{12}-\left(\frac{\sigma}{r}\right)^{6}\right]
$$

wobei $\epsilon$ und $\sigma$ die Lennard-Jones Parameter und $r$ der Teilchenabstand ist. Die Punktdipol Wechselwirkung $u_{\mathrm{D}}$ wird gemäß

$$
u_{\mathrm{D}}\left(\mathbf{r}_{\mathrm{ij}}, \omega_{\mathrm{i}}, \omega_{\mathrm{j}}, \mu\right)=\frac{1}{4 \pi \epsilon_{0}} \cdot \frac{\mu^{2}}{\left|\mathbf{r}_{\mathrm{ij}}\right|^{3}} f_{\mathrm{D}}\left(\omega_{\mathrm{i}}, \omega_{\mathrm{j}}\right)
$$

erhalten. $\mathbf{r}_{\mathrm{ij}}$ ist der Schwerpunktsabstand zwischen den Teilchen i und $\mathbf{j}, \mu$ das Dipolmoment, $\epsilon_{0}$ die Dielektrizitätskonstante, $\omega_{\mathrm{i}}$ und $\omega_{\mathrm{j}}$ sind die Orientierungen von Teilchen i und $\mathrm{j}$, der Orientierungsfaktor $f_{\mathrm{D}}\left(\omega_{\mathrm{i}}, \omega_{\mathrm{j}}\right)$ ist in [124] gegeben.

Für Fluoroform wird zusätzlich zu der Punktdipol Wechselwirkung das 2-Zentren Lennard-Jones Potential $u_{2 \mathrm{CLJ}}$

$$
u_{2 \mathrm{CLJ}}\left(\mathbf{r}_{\mathrm{ij}}, \omega_{\mathrm{i}}, \omega_{\mathrm{j}}, L\right)=\sum_{a=1}^{2} \sum_{b=1}^{2} 4 \epsilon\left[\left(\frac{\sigma}{r_{a b}}\right)^{12}-\left(\frac{\sigma}{r_{a b}}\right)^{6}\right]
$$




\begin{tabular}{l|c|c|c|c|c} 
Stoff & $\sigma / \AA$ & $\left(\epsilon / k_{\mathrm{B}}\right) / \mathrm{K}$ & $L / \AA$ & $\mu / \mathrm{D}$ & $Q / \mathrm{D} \AA$ \\
\hline $\mathrm{Xe}[121]$ & 3.9011 & 227.55 & - & 0 & 0 \\
$\mathrm{CO}_{2}[121]$ & 2.9847 & 133.22 & 2.4176 & 0 & 3.7938 \\
$\mathrm{C}_{2} \mathrm{H}_{6}[121]$ & 3.4896 & 136.99 & 2.3762 & 0 & 0.8277 \\
$\mathrm{CHF}_{3}[123]$ & 3.2643 & 123.56 & 2.5670 & 2.1607 & 0 \\
$\mathrm{CH}_{2} \mathrm{I}_{2}[123]$ & 5.0481 & 387.14 & - & 4.8971 & 0
\end{tabular}

Tabelle 5.1: Potentialparameter.

verwendet. $L$ ist der Abstand der beiden Lennard-Jones Zentren, $r_{\mathrm{ab}}$ ist einer der vier zwischenmolekularen Lennard-Jones Zentrumsabstände; $a$ zählt die beiden Seiten von Teilchen i, $b$ die von Teilchen $\mathrm{j}$.

Kohlendioxid und Ethan werden durch ein 2-Zentren Lennard-Jones Potential sowie eine Punktquadrupol Wechselwirkung $u_{\mathrm{Q}}$ mit dem Quadrupolmoment Q beschrieben:

$$
u_{\mathrm{Q}}\left(\mathbf{r}_{\mathrm{ij}}, \omega_{\mathrm{i}}, \omega_{\mathrm{j}}, Q^{2}\right)=\frac{3}{4} \frac{Q^{2}}{\left|\mathbf{r}_{\mathrm{ij}}\right|^{5}} f_{\mathrm{Q}}\left(\omega_{\mathrm{i}}, \omega_{\mathrm{j}}\right) .
$$

Der Orientierungsfaktor $f_{\mathrm{Q}}\left(\omega_{\mathrm{i}}, \omega_{\mathrm{j}}\right)$ ist ebenfalls in [124] gegeben.

Xenon wird mit einem einfachen Lennard-Jones Potential nach G1.5.1 modelliert. Alle verwendeten Potentialparameter sind in Tabelle 5.1 zusammengefasst.

Für die Diiodmethan-Lösungsmittelwechselwirkungen wurden die Lennard-Jones Parameter gemäß der Lorentz-Berthelot Mischregeln [86]

$$
\sigma_{12}=\frac{1}{2}\left(\sigma_{1}+\sigma_{2}\right)
$$

und

$$
\epsilon_{12}=\left(\epsilon_{1} \epsilon_{2}\right)^{\frac{1}{2}}
$$

erhalten. Alle Dipole und Quadrupole wurden durch Partialladungen und damit durch Coulomb Wechselwirkungen ausgedrückt:

- $\mathrm{CH}_{2} \mathrm{I}_{2}$ : Das Dipolmoment wird durch zwei Partialladungen $|q|=0.9725$ e (e: Elementarladung) dargestellt. Die positive Ladung liegt auf dem C-Atom, die negative im Molekülschwerpunkt, der Abstand der beiden Ladungen beträgt 1.048395 ̊. Das Lennard-Jones Zentrum liegt auf dem Schwerpunkt.

- $\mathrm{CO}_{2}$ : Das Quadrupolmoment wird durch drei im Abstand von $L / 2$ linear angeordneten Partialladungen $q_{1}=-0.13514 e, q_{2}=-2 q_{1}$ und $q_{3}=q_{1}$ repräsentiert. Die beiden negativen Ladungen liegen dabei auf den Lennard-Jones Zentren.

- $\mathrm{C}_{2} \mathrm{H}_{6}$ : Das Quadrupolmoment wird durch drei im Abstand von $L / 2$ linear angeordneten Partialladungen $q_{1}=-0.031 e, q_{2}=-2 q_{1}$ und $q_{3}=q_{1}$ repräsentiert. Ebenso wie bei Kohlendioxid liegen die negativen Ladungen auf den Lennard-Jones Zentren.

- $\mathrm{CHF}_{3}$ : Das Dipolmoment wird durch zwei im Abstand von $L$ angeordnete Ladungen $q_{1}=-q_{2}=0.175$ e dargestellt. Die Partialladungen liegen auf den Lennard-Jones Zentren. 
Die MD-Simulationen wurden mit dem MOLDY Programmpaket [125] durchgeführt. Die Simulationsboxen bestanden jeweils aus einem Diiodmethanmolekül und 200 Lösungsmittelmolekülen. Der cut off-Radius betrug $5 \sigma_{\text {Lösungsmittel }}$. Bei niedrigen Dichten wurde mit Integrationsschritten von $2 \mathrm{fs}$, bei höheren mit $1 \mathrm{fs}$ gerechnet. Jedes System wurde zunächst 200 ps lang äquilibriert, um anschließend eine 2 ns dauernde Trajektorie zu berechnen. Obwohl die Potentiale von Diiodmethan in $\mathrm{CO}_{2}, \mathrm{C}_{2} \mathrm{H}_{6}$ und $\mathrm{CHF}_{3}$ anisotrop sind, wurde statt der Paar-Korrelationsfunktion die radiale Verteilungsfunktion $g(r)$ berechnet. Der Abstand $r$ entspricht hierbei den Abständen der Molekülschwerpunkte.

\subsection{Radiale Verteilungsfunktion von $\mathrm{CH}_{2} \mathrm{I}_{2}$ in $\mathrm{Xe}$}

Es wurden radiale Verteilungsfunktion in dem experimentell untersuchten Dichtebereich 15-22 mol/l im Abstand von $1 \mathrm{~mol} / \mathrm{l}$ berechnet. Die simulierte Temperatur betrug $35^{\circ} \mathrm{C}$. Abb.5.1 zeigt alle erhaltenen Verteilungsfunktionen. Bei größeren Abständen nähern sich alle Funktionen einem Wert von eins an, was einer vollständig statistischen Verteilung und damit der mittleren homogenen Lösungsmitteldichte entspricht. Nach Druckerhöhung steigt das Maximum der ersten Lösungsmittelschale an und die Schale verschiebt sich zu kürzeren Abständen. Zudem erscheint das Maximum der zweiten Lösungsmittelschale nach Dichteerhöhung ausgeprägter.

Als Maß für die mittlere lokale Dichte wird das Maximum der ersten Lösungsmittelschale $g_{\max }$ verwendet. Weil die radiale Verteilungsfunktion auf eine vollständig statistische Verteilung normiert ist, muss dieser Wert mit der mittleren homogenen Lösungsmitteldichte multipliziert werden: $\rho_{12}=g_{\max } \cdot \rho$. Die dichteabhängigen lokalen Dichten sind in Abb. 5.2 aufgetragen. Im höheren Dichtebereich verlaufen die lokalen Dichten, wie in Abschn.2.4 beschrieben, linear mit der homogenen Dichte. Für diesen linearen Zusammenhang sind nur die repulsiven Wechselwirkungen verantwortlich [81]. Im niedrigeren Dichtebereich weichen die mittleren lokalen Dichten durch die attraktiven Wechselwirkungen von diesem linearen Verlauf zu größeren Werten ab, das System $\mathrm{CH}_{2} \mathrm{I}_{2}$-Xe zeigt somit in dem experimentell untersuchten Dichtebereich eine lokale Dichtevergrößerung.

\subsection{Radiale Verteilungsfunktion von $\mathrm{CH}_{2} \mathrm{I}_{2}$ in $\mathrm{CO}_{2}$}

Es wurden radiale Verteilungsfunktionen von 10-28 mol/l im Abstand von $2 \mathrm{~mol} / \mathrm{l}$ bei beiden experimentellen Temperaturen berechnet. Abb.5.3 zeigt einige der simulierten Verteilungsfunktionen für $35^{\circ} \mathrm{C}$, in Abb. 5.4 ist das entsprechende Bild für $50^{\circ} \mathrm{C}$ dargestellt. In beiden Auftragungen zeigen sich ähnliche Trends wie bei den Verteilungsfunktionen für Xe: Nach Dichteerhöhung verschiebt die erste Lösungsmittelschale zu kürzeren Abständen und die zweite Lösungsmittelschale wächst heraus. Jedoch fällt mit zunehmender Dichte das Maximum der ersten Schale ab. Zudem weist die erste Schale bei den höchsten simulierten Dichten zwei Maxima auf. Das niedrigere Maximum bei kürzen Abständen entspricht einer Geometrie bei der das $\mathrm{CO}_{2}$-Molekül seitlich an das $\mathrm{CH}_{2} \mathrm{I}_{2}$ angelagert ist. Das zweite, höhere Maximum gehört zu einer terminalen Anlagerung. 


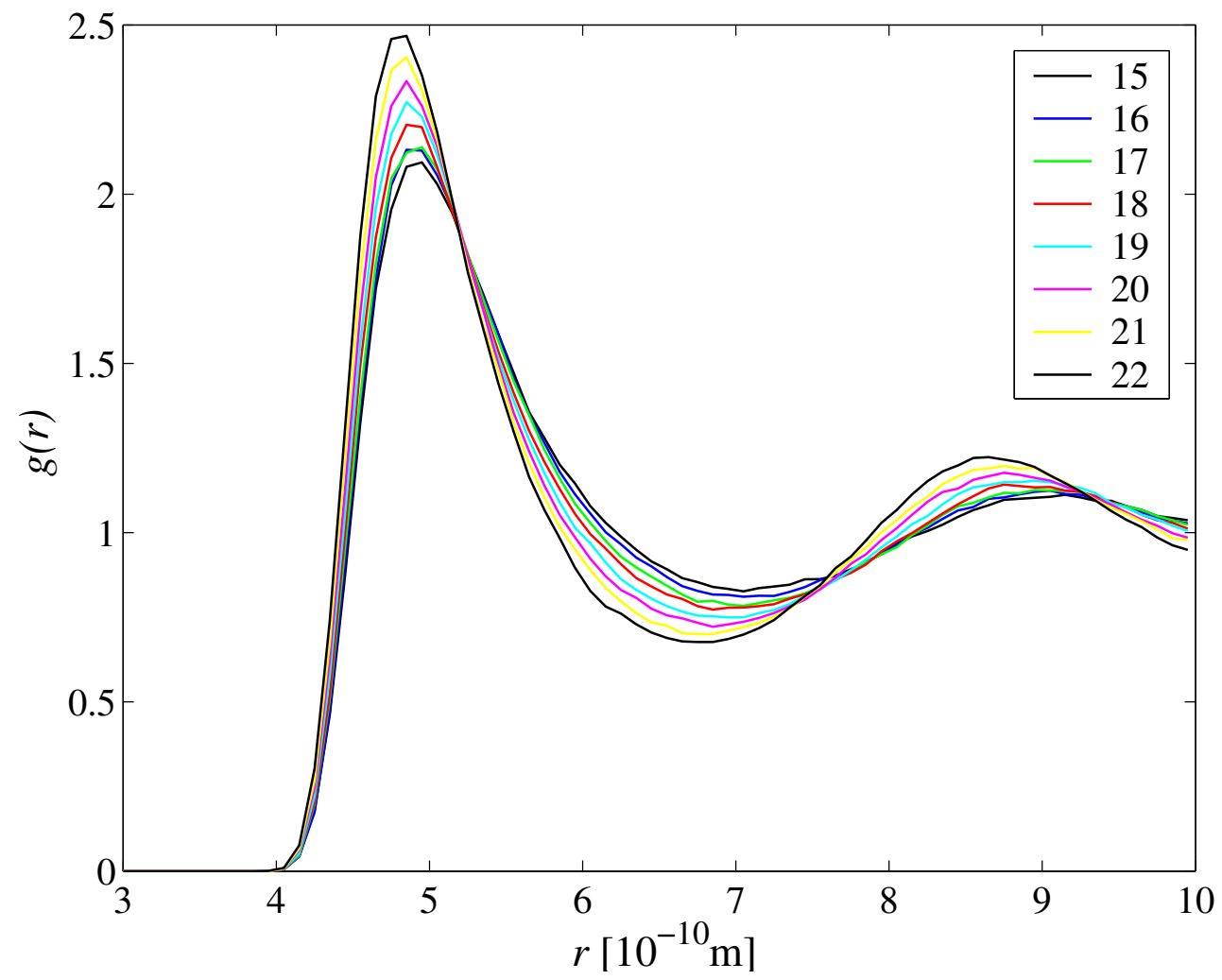

Abbildung 5.1: Dichteabhängige radiale Verteilungsfunktionen von $\mathrm{CH}_{2} \mathrm{I}_{2}$ in $\mathrm{Xe}$ bei $35^{\circ} \mathrm{C}$.

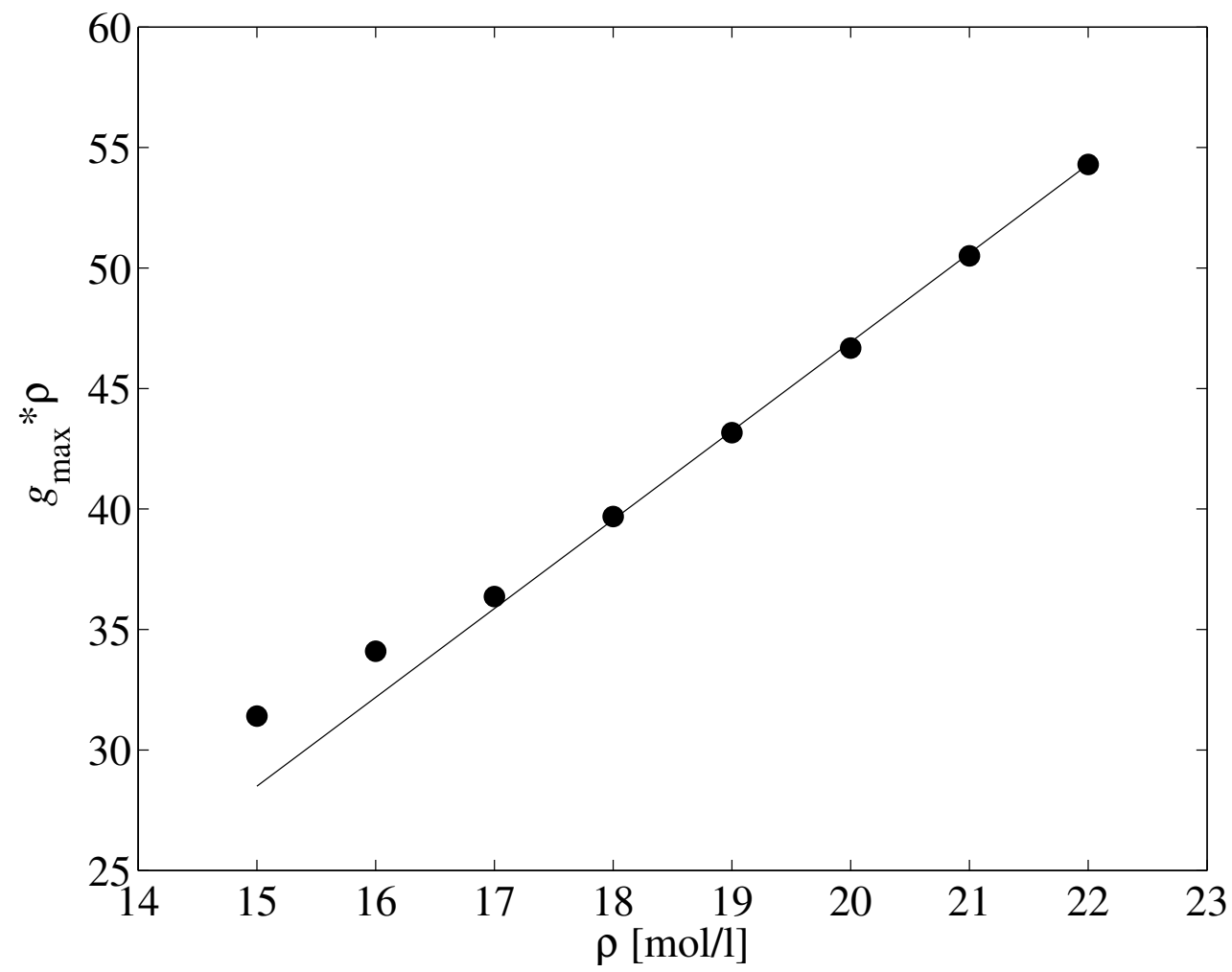

Abbildung 5.2: Dichteabhängige lokale Dichten von $\mathrm{CH}_{2} \mathrm{I}_{2}$ in Xe. 


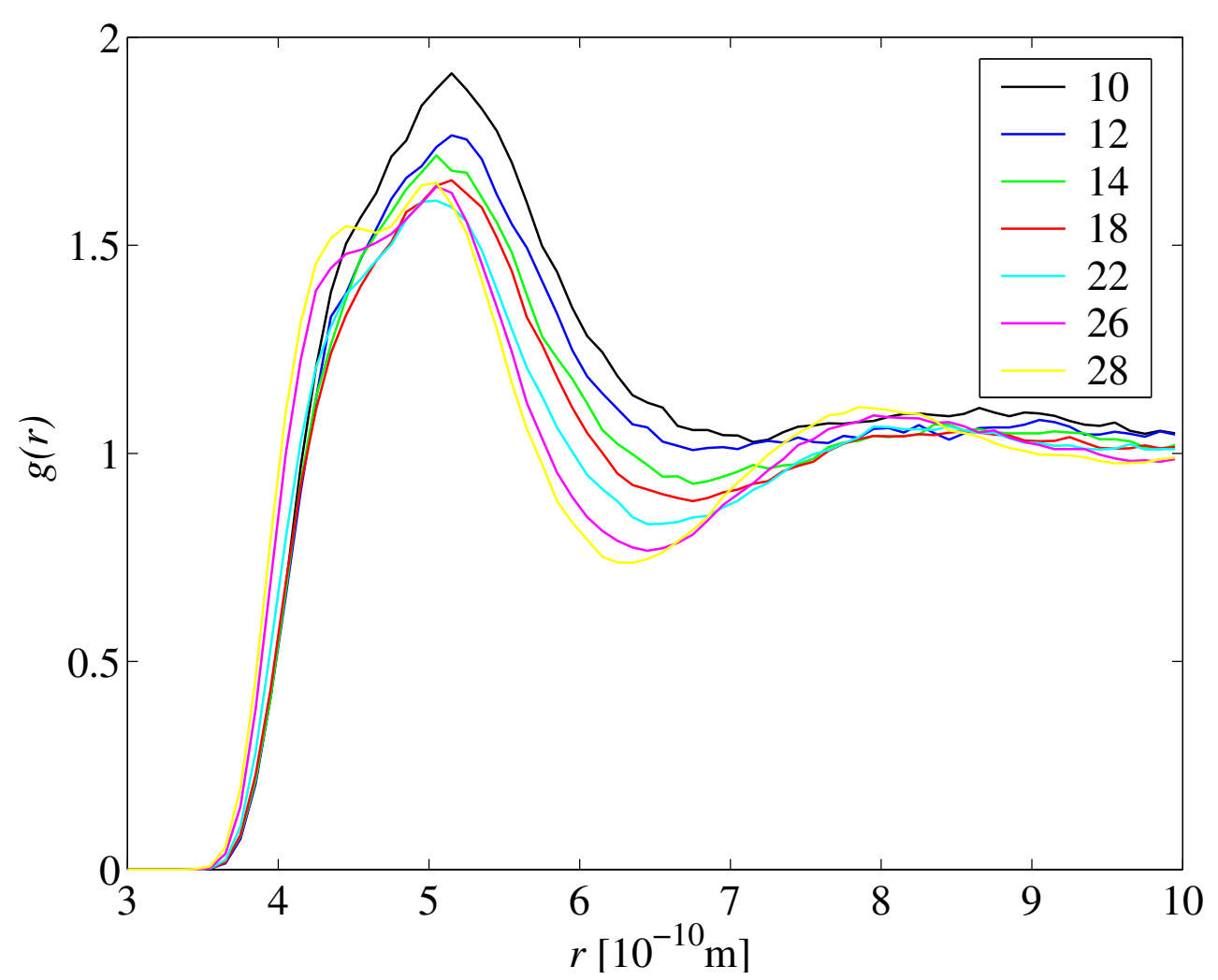

Abbildung 5.3: Dichteabhängige radiale Verteilungsfunktionen von $\mathrm{CH}_{2} \mathrm{I}_{2}$ in $\mathrm{CO}_{2}$ bei $35^{\circ} \mathrm{C}$.

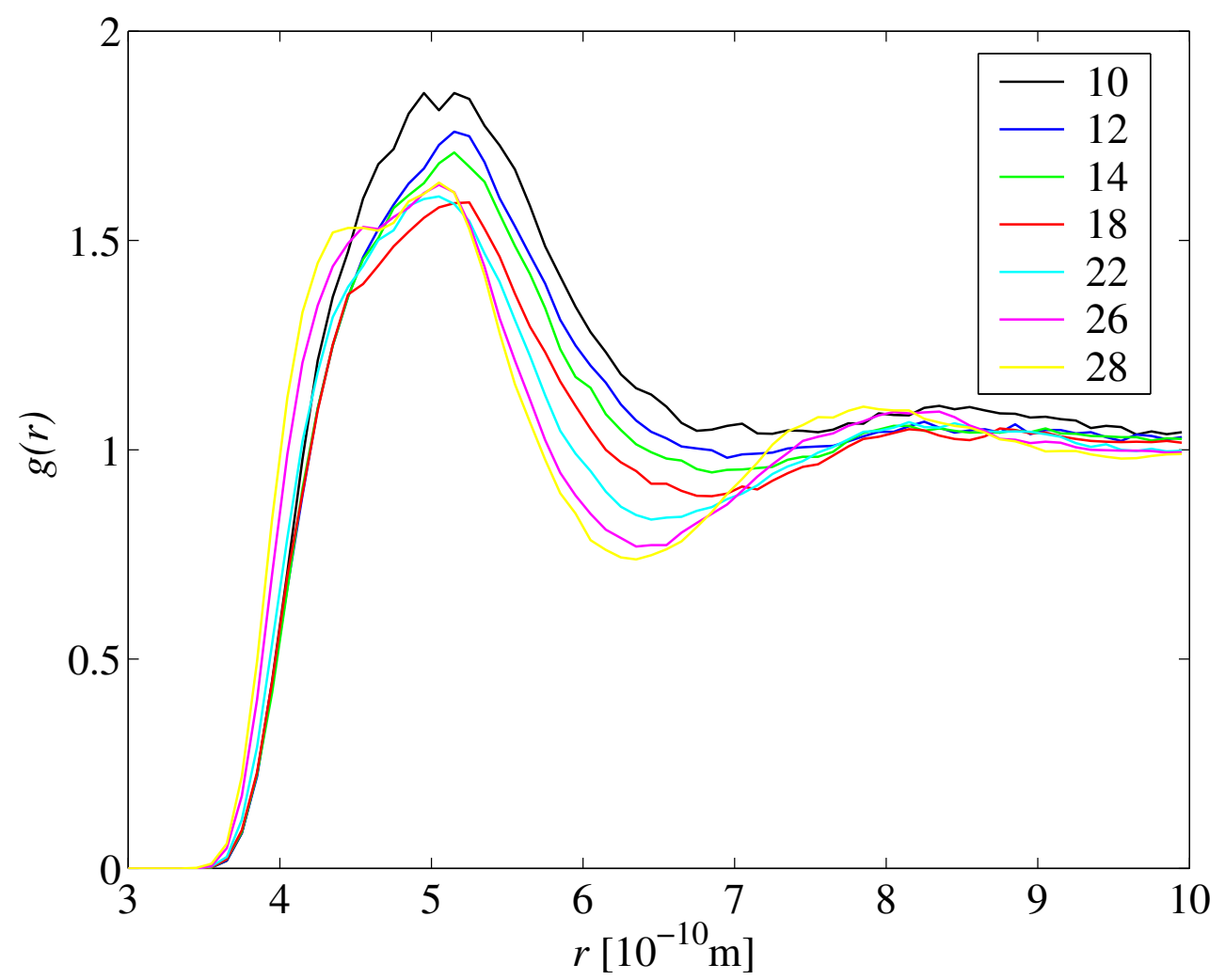

Abbildung 5.4: Dichteabhängige radiale Verteilungsfunktionen von $\mathrm{CH}_{2} \mathrm{I}_{2}$ in $\mathrm{CO}_{2}$ bei $50{ }^{\circ} \mathrm{C}$. 


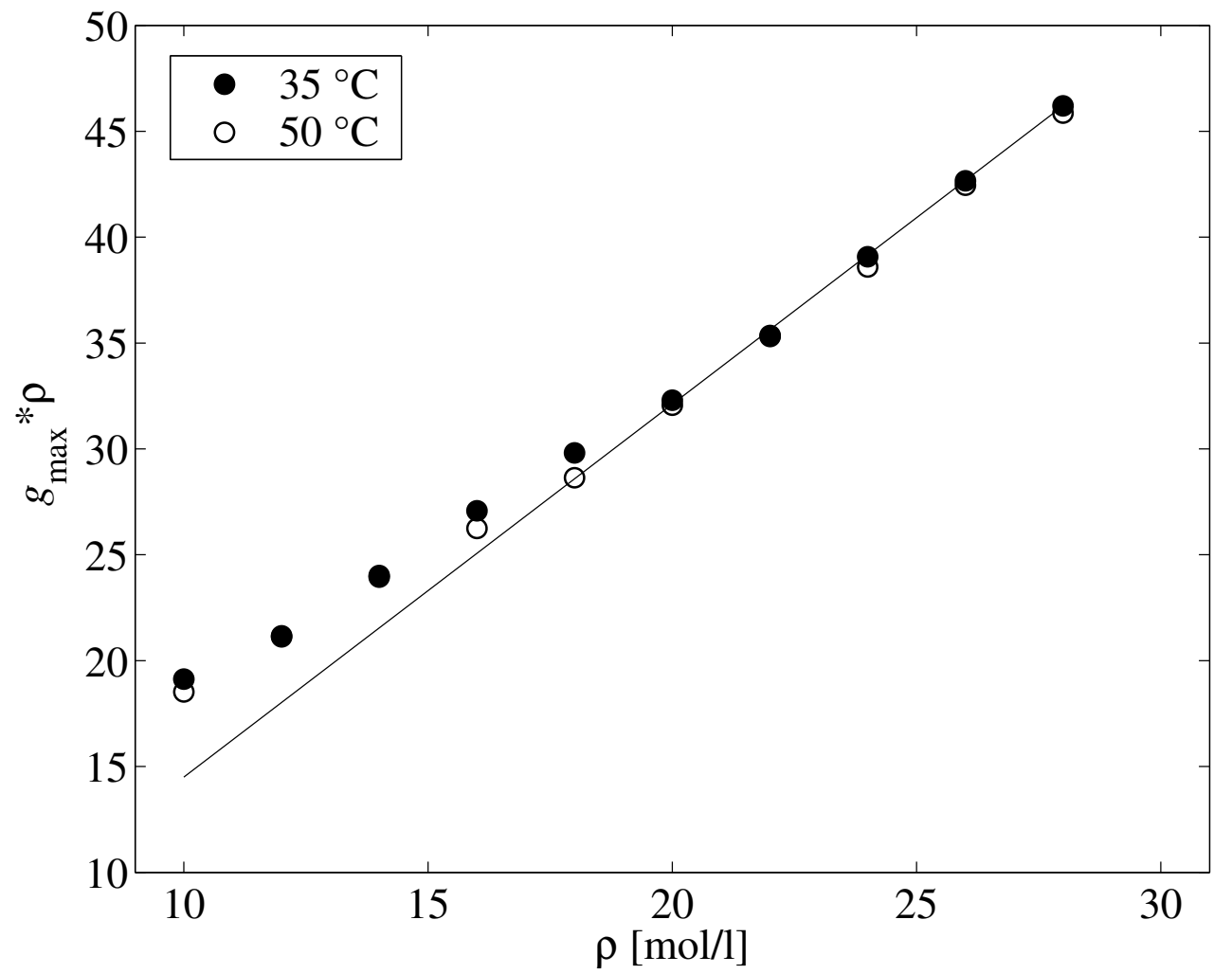

Abbildung 5.5: Vergleich der lokalen Dichten von $\mathrm{CH}_{2} \mathrm{I}_{2}$ in $\mathrm{CO}_{2}$ bei 35 und $50^{\circ} \mathrm{C}$.

In Abb.5.5 werden die lokalen Dichten beider Temperaturen miteinander verglichen. Die Werte für $50^{\circ} \mathrm{C}$ liegen geringfügig unter denen von $35^{\circ} \mathrm{C}$, zeigen ansonsten aber einen identischen Verlauf. Die lokale Dichtevergrößerung im unteren Dichtebereich ist wie im Falle des Xe gut sichtbar.

\subsection{Radiale Verteilungsfunktion von $\mathrm{CH}_{2} \mathrm{I}_{2}$ in $\mathrm{C}_{2} \mathrm{H}_{6}$}

Der modellierte Dichtebereich liegt zwischen 10-20 mol/l, die Verteilungsfunktionen wurden im Abstand von $1 \mathrm{~mol} / \mathrm{l}$ errechnet. Abb.5.6 zeigt einige der Verteilungsfunktionen für $36^{\circ} \mathrm{C}$. Es zeigt sich ein qualitativ ähnliches Verhalten wie für Xe: Anwachsen des Maximums und Verschiebung der ersten Lösungsmittelschale zu kürzeren Abständen sowie Herausbildung der zweiten Lösungsmittelschale.

Die Auftragung 5.7 zeigt ebenfalls bei den niedrigen Dichten eine lokale Dichtevergrößerung. 


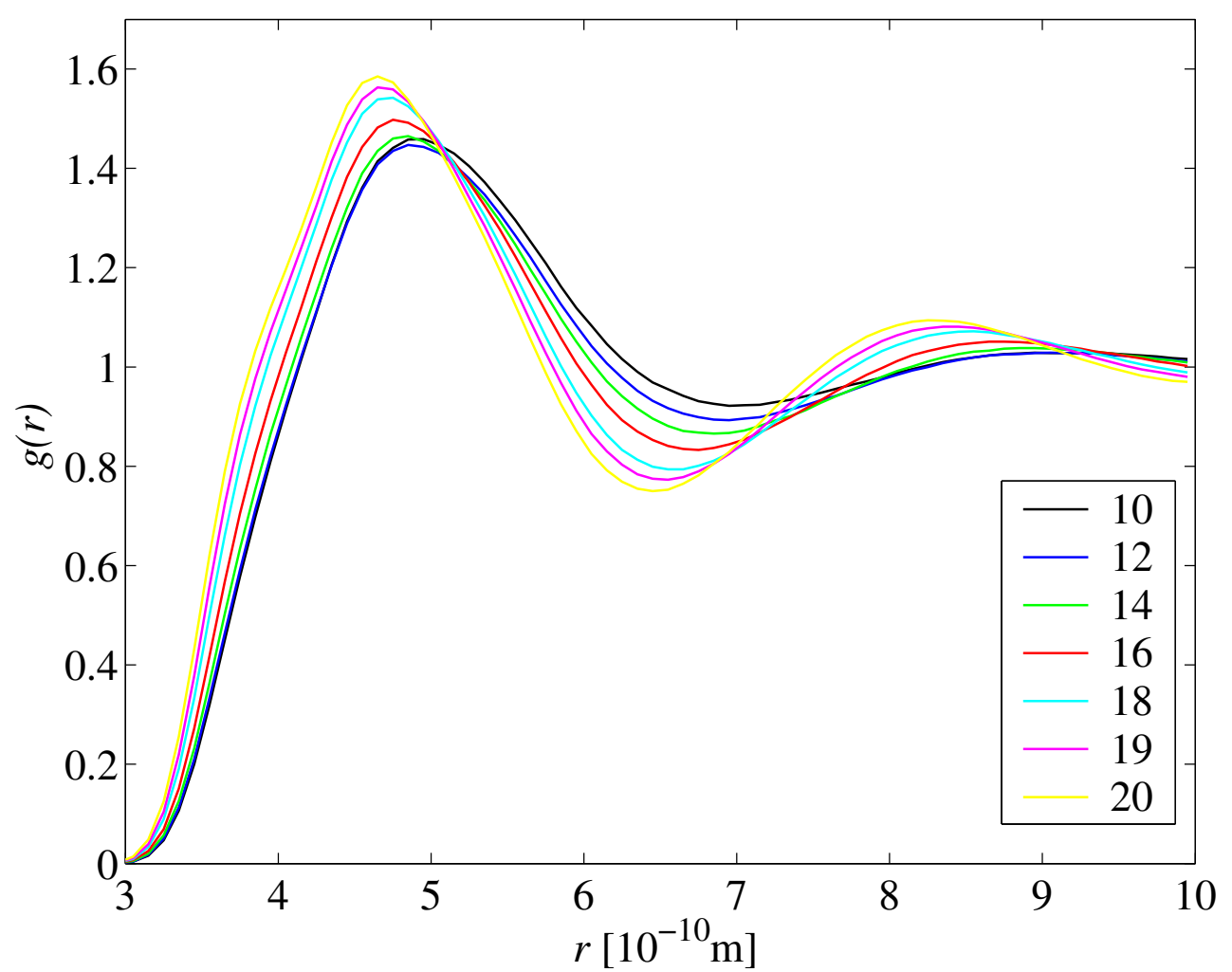

Abbildung 5.6: Dichteabhängige radiale Verteilungsfunktionen von $\mathrm{CH}_{2} \mathrm{I}_{2}$ in Ethan bei $36{ }^{\circ} \mathrm{C}$

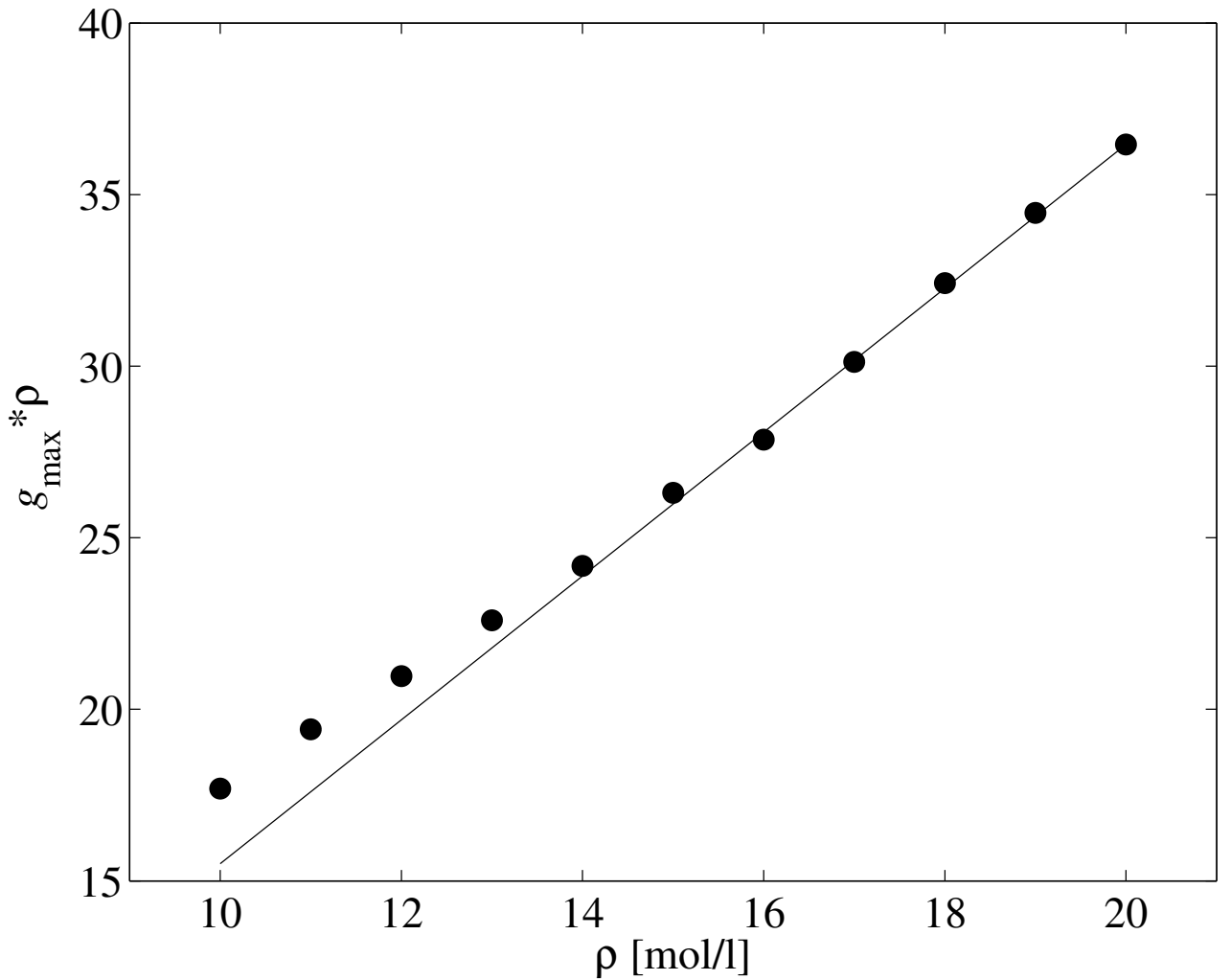

Abbildung 5.7: Dichteabhängige lokale Dichten von $\mathrm{CH}_{2} \mathrm{I}_{2}$ in Ethan. 


\subsection{Radiale Verteilungsfunktion von $\mathrm{CH}_{2} \mathrm{I}_{2}$ in $\mathrm{CHF}_{3}$}

Der untersuchte Dichtebereich entspricht dem des Ethans, die simulierte Temperatur beträgt $30^{\circ} \mathrm{C}$. Die radialen Verteilungsfunktionen in Abb.5.8 zeigen ein mit zunehmender Dichte abfallendes Maximum der ersten Lösungsmittelschale. Die zweite Lösungsmittelschale ist auch bei den höchsten simulierten Dichten kaum ausgeprägt.

Die lokalen Dichten des polaren Lösungsmittels Fluoroform in Abb.5.9 zeigen zu niedrigen Dichten eine besonders ausgeprägte Vergrößerung.

\subsection{Zusammenfassung}

Abb.5.10 zeigt die Maxima aller simulierten radialen Verteilungsfunktionen. Die reduzierten Temperaturen betrugen 1.01 für die polyatomaren Lösungsmittel und 1.06 für Xenon. Dabei weisen die Kurven für die polyatomaren Lösungsmittel einen gekrümmten Verlauf mit einem Minimum um die reduzierte Dichte von 2 auf, während Xenon im untersuchten Dichtebereich monoton mit zunehmender Dichte ansteigt. Die Tatsache, dass bei den polyatomaren Fluiden die Maxima um den kritischen Punkt herum größer als bei hohen Dichten ausfallen ist auf die vorher diskutierte lokale Dichtevergrößerung zurückzuführen. In dieser Auftragung ist besonders deutlich zu erkennen, dass das polare Fluoroform die größte lokale Dichtevergrößerung aller verwendeten Lösungsmittel zeigt.

In Abb.5.11 werden die lokalen Dichten von Diiodmethan in den verschiedenen Lösungsmitteln verglichen. Die lokalen Dichten wurden in dieser Auftragung gemäß $\rho_{12}=g_{\max } \cdot \rho_{\mathrm{r}}$ erhalten. Wiederum ergibt sich ein sehr ähnlicher Verlauf für die polyatomaren Lösungsmittel während Xenon ein abweichendes Verhalten aufweist. Die lokalen Dichten für Xenon liegen im Dichtebereich $\rho_{\mathrm{r}}>2$ über denen der anderen Lösungsmittel und weisen eine wesentlich stärkere Dichteabhängigkeit auf, was durch die größeren repulsiven Wechselwirkungen beschreibenden Lennard-Jones Durchmesser (siehe Tabelle5.1) zu erklären ist. 


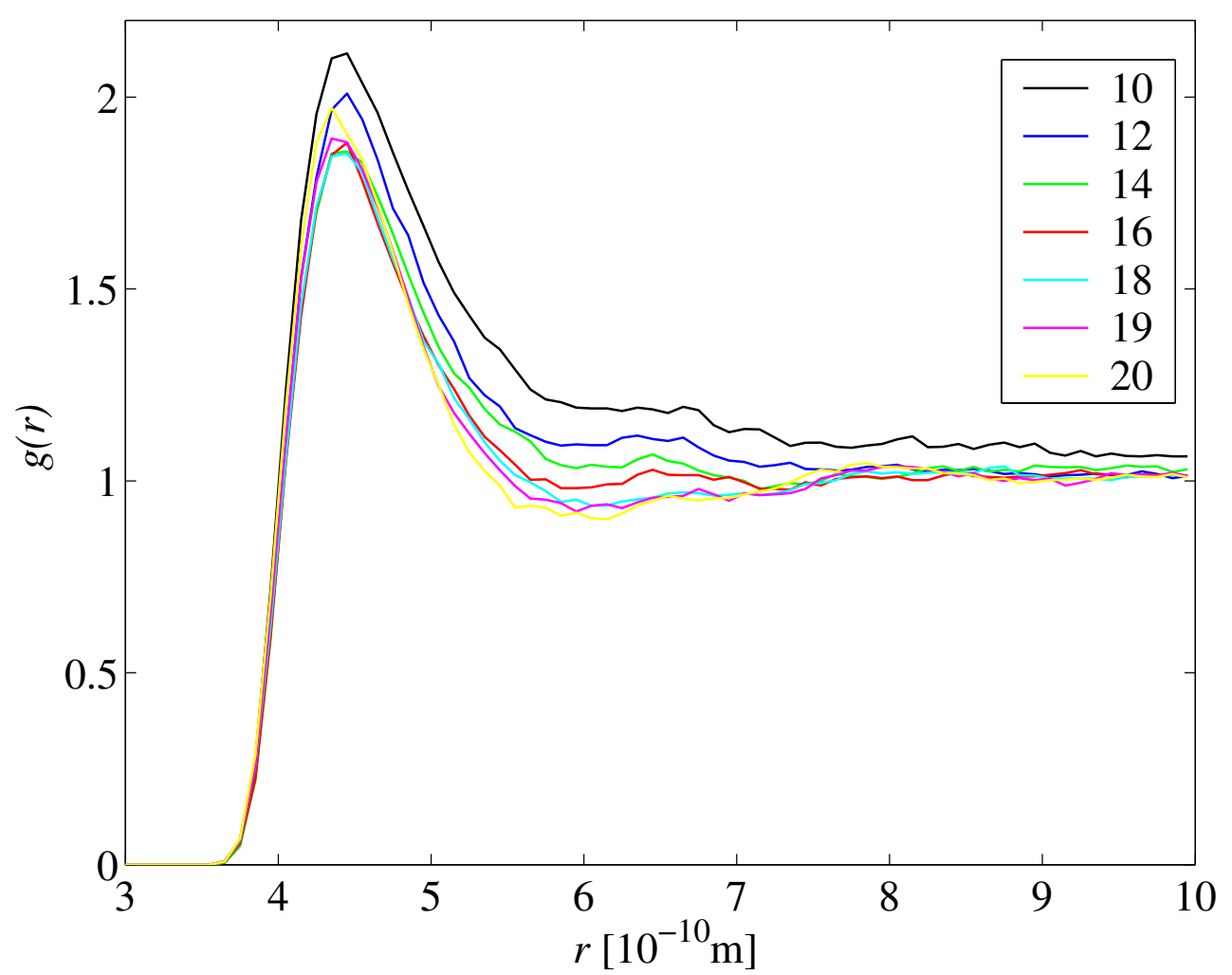

Abbildung 5.8: Dichteabhängige radiale Verteilungsfunktionen von $\mathrm{CH}_{2} \mathrm{I}_{2}$ in $\mathrm{CHF}_{3}$ bei $30^{\circ} \mathrm{C}$

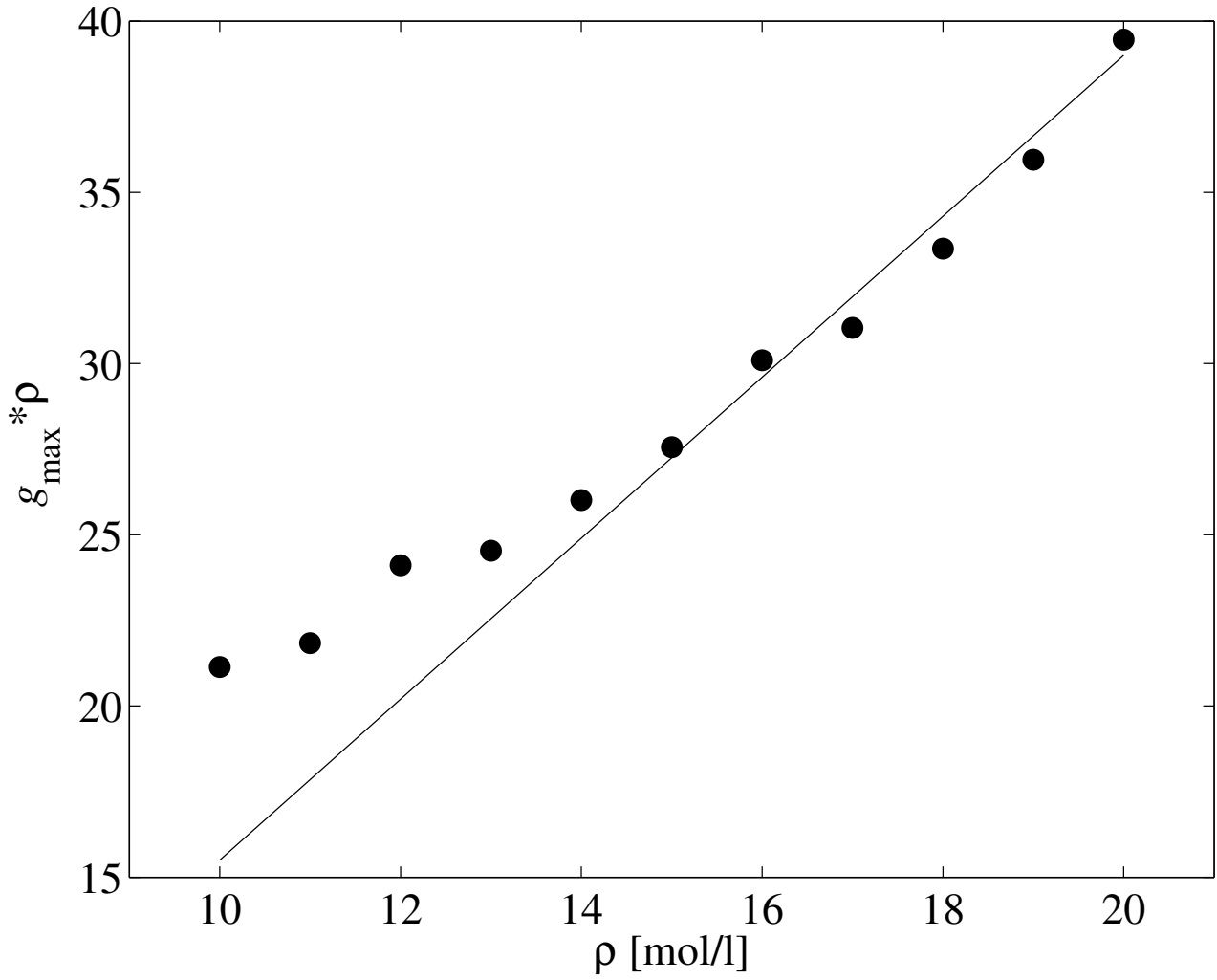

Abbildung 5.9: Dichteabhängige lokale Dichten von $\mathrm{CH}_{2} \mathrm{I}_{2}$ in $\mathrm{CHF}_{3}$. 


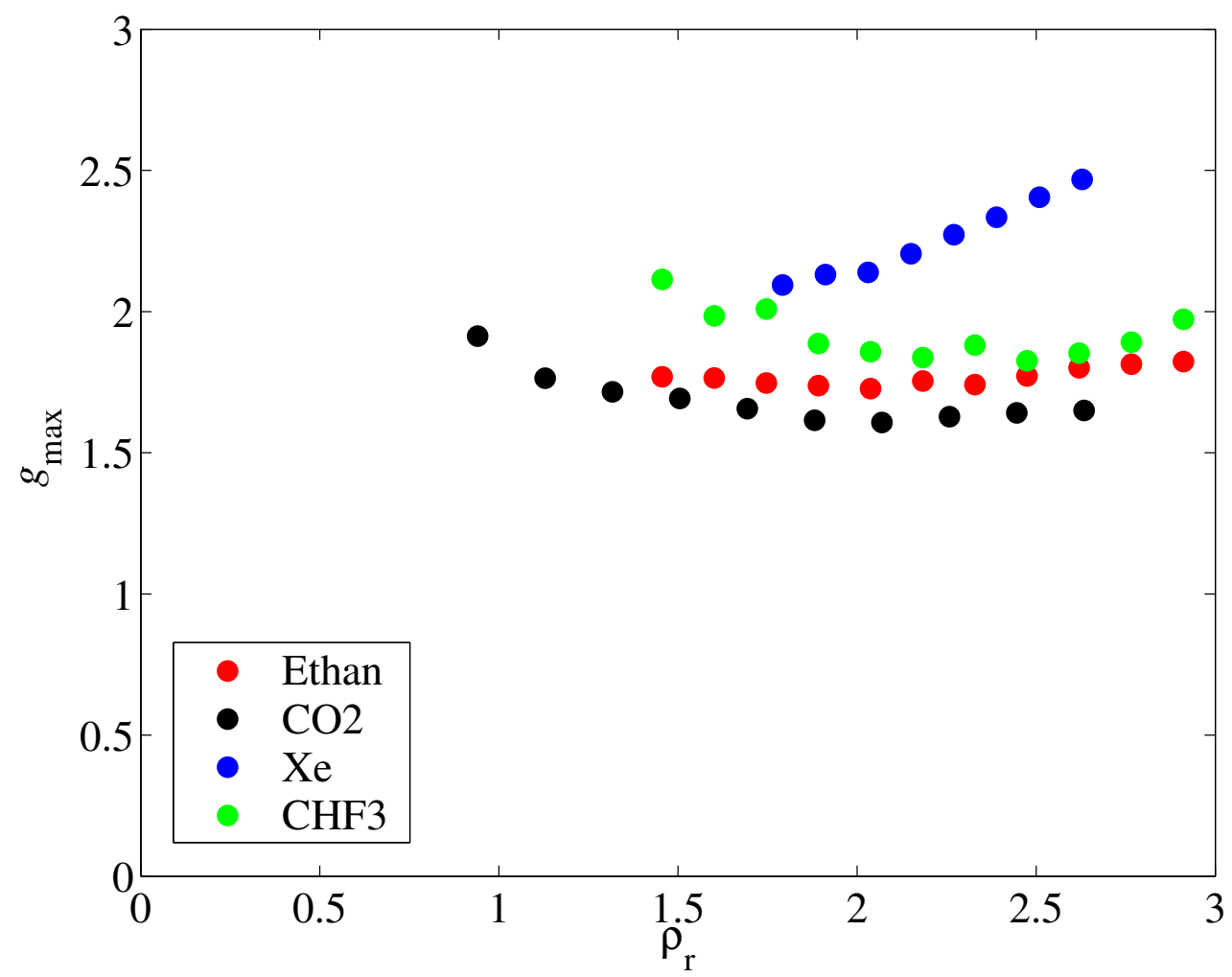

Abbildung 5.10: Maxima der radialen Verteilungsfunktionen in den verschiedenen Lösungsmitteln.

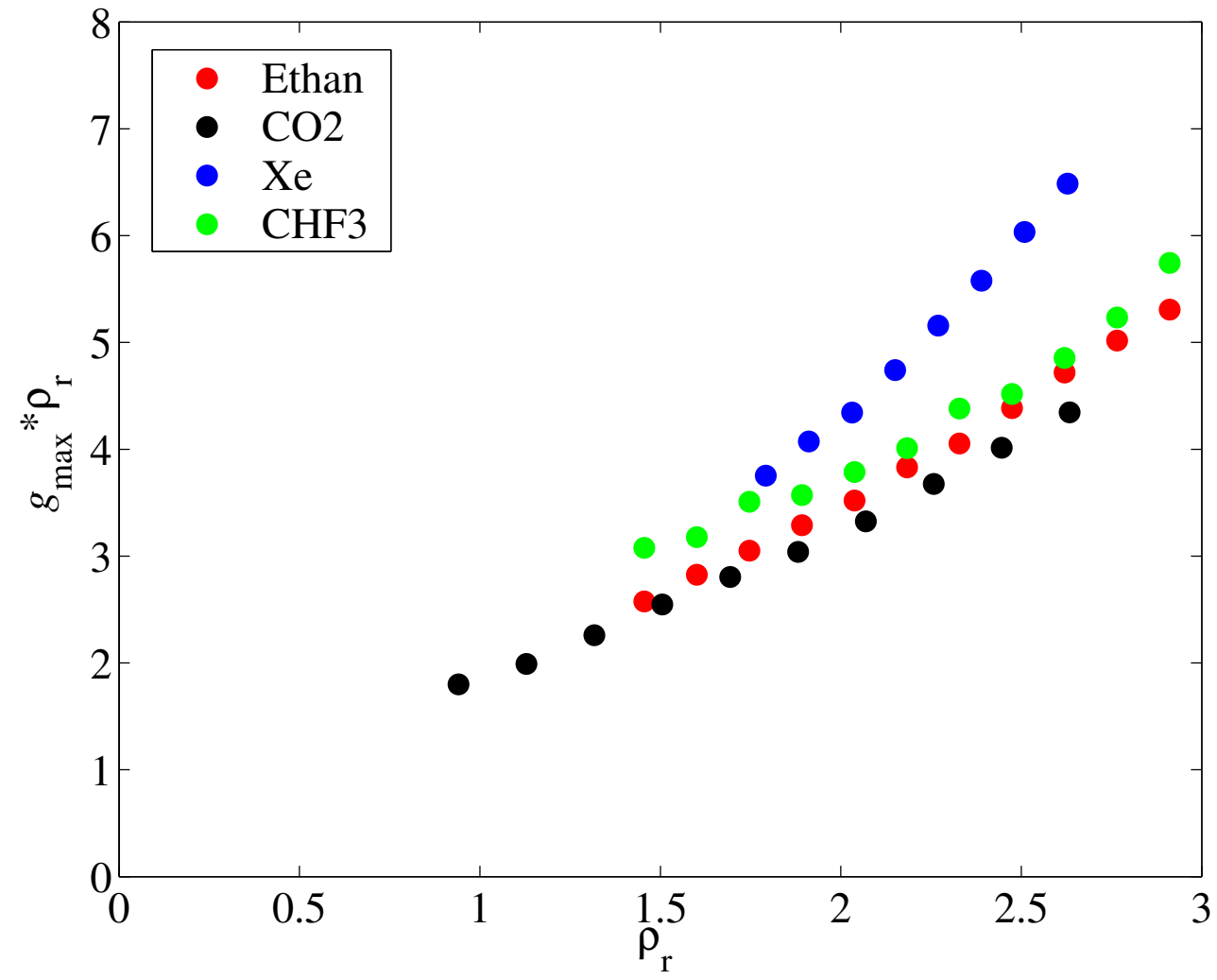

Abbildung 5.11: Lokale Dichten in den verschiedenen Lösungsmitteln. 


\section{Diskussion}

\subsection{Zuordnung im Kurzzeitbereich}

Die Aufklärung des Mechanismus der Photoisomerisierung von Diiodmethan mit Hilfe der in dieser Arbeit gemessenen Absorptionszeitprofile ist nur in dem Maße möglich, wie die Transienten mit den absorbierenden molekularen Spezies identifiziert werden können. Wie bereits in Abschn.2.2.2 dargestellt, wird die Absorption zu längeren Verzögerungszeiten dem $\mathrm{CH}_{2} \mathrm{I}$ - I-Isomer zugeordnet. Die Zuordnung im Kurzzeitbereich ist weniger eindeutig: Das Anfangsmaximum wird mit der transienten Absorption aus dem $S_{1}$-Zustand erklärt, das lokale Minimum nach Dissoziation wird einer schnellen Isomerbildung zugeschrieben [63]. Im Folgenden sollen Alternativen zu dieser Interpretation diskutiert werden.

\subsubsection{Franck-Condon Region des angeregten $\mathrm{CH}_{2} \mathrm{I}_{2}$}

In der Vorstellung von Tarnovsky etal. 61] befindet sich das direkt nach Photoanregung auf der repulsiven $S_{1}$-Potentialfläche gebildetete Wellenpaket in einem Bereich, der eine experimentell beobachtbare Absorption in noch höhere Energieniveaus ermöglicht. Der anschließende Abfall (siehe Abb.4.15) spiegelt demnach die Dissoziation des Diiodmethans in Lösung wider. In der Gasphase beträgt die Dissoziationszeit $\tau_{\text {dis }}<100$ fs (siehe Abschn.2.2.1).

Es stellt sich die Frage, ob und in welchem Maße die Lösungsmittelviskosität die Dissoziation, d. h. die Wellenpaketbewegung auf der repulsiven Potentialfläche, dämpft. Für Isomerisierungen, die über keine oder eine sehr niedrige Barriere ablaufen, wie z. B. die Isomerisierung von cis-Stilben, wird eine Viskositätsabhängigkeit der Geschwindigkeitskonstanten $k$ der Form $k \propto 1 / \eta^{a}$ mit $a \leq 1$ erwartet [126]. Es konnte wegen der zu geringen Zeitfauflösung (200-300 fs) im experimentell untersuchten Viskositätsbereich $(25-160 \mu \mathrm{Pa} \cdot \mathrm{s})$ jedoch keine Abhängigkeit von $\tau_{\text {dis }}$ aufgelöst werden (vergl. Abb.4.16).

\subsubsection{Direkte Isomerbildung}

Tarnovsky et al. ordneten die Absorption bei $\sim 1$ ps schnell gebildetem Isomer zu, was durch die Ähnlichkeit der Spektren zu diesen Verzögerungszeiten mit dem Langzeitspektrum erklärt wurde (siehe Abschn.2.2.2). Das Anwachsen des normierten Minimums $A_{\text {min,n }}$ mit zunehmender Dichte (siehe Abb.4.12 und 4.19) unterstützt diese These, weil ein dichter werdender Lösungsmittelkäfig den mit der Isomerbildung konkurrierenden Käfigausbruch erschwert und somit die Ausbeute an Isomer erhöht. Jedoch sprechen energetische Argumente gegen diese Interpretation: Die innere Energie $\left\langle E_{\mathrm{CH}_{2} \mathrm{I}}\right\rangle$ des $\mathrm{CH}_{2} \mathrm{I}$-Radikals nach Anregung mit $\lambda=304 \mathrm{~nm}$ kann unter der Annahme, dass $20 \%$ der Überschussenergie in 


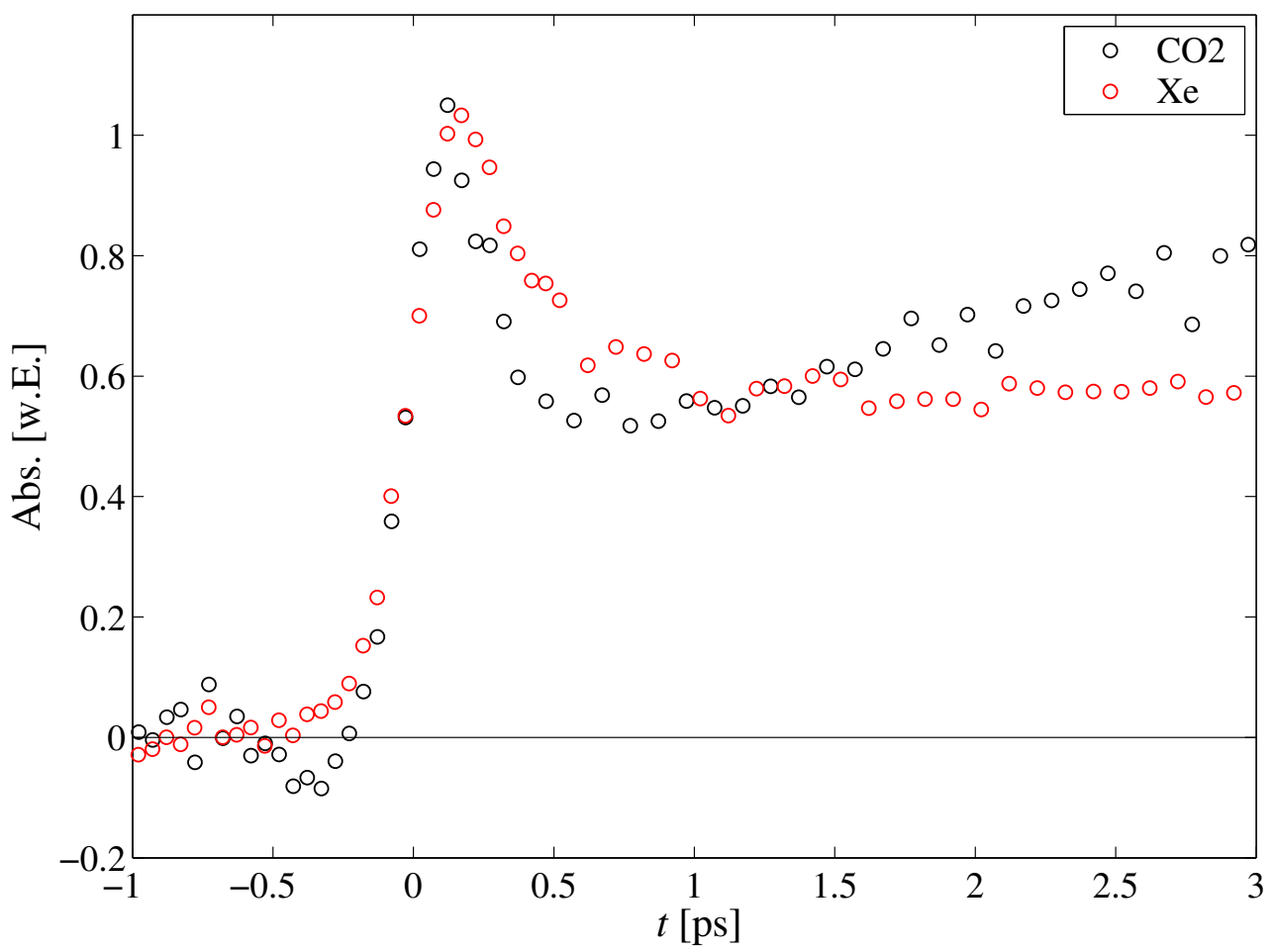

Abbildung 6.1: Absorptionszeitprofile $\mathrm{zu}$ frühen Verzögerungszeiten von $\mathrm{CH}_{2} \mathrm{I}_{2}$ in $\mathrm{CO}_{2}$ $\left(35^{\circ} \mathrm{C}, \rho_{\mathrm{r}}=2.2\right)$ und $\mathrm{Xe}\left(35^{\circ} \mathrm{C}, \rho_{\mathrm{r}}=2.0\right)$ bei $405 \mathrm{~nm}$ Abfrage.

die Translationsfreiheitsgrade fließt (siehe Abschn.2.2.1), mit Hilfe der Standardbildungsenthalpien bei $298 \mathrm{~K}\left(\Delta_{\mathrm{f}} H\left(\mathrm{I}\left({ }^{2} P_{3 / 2}\right)\right)=106.8 \mathrm{~kJ} / \mathrm{mol}, \Delta_{\mathrm{f}} H\left(\mathrm{CH}_{2} \mathrm{I}_{2}(g)\right)=119.5 \mathrm{~kJ} / \mathrm{mol}\right.$ [54], $\left.\Delta_{\mathrm{f}} H\left(\mathrm{CH}_{2} \mathrm{I}\right)=217.6 \mathrm{~kJ} / \mathrm{mol}[55]\right)$ gemäß $\left\langle E_{\mathrm{CH}_{2} \mathrm{I}}\right\rangle=0.8 \cdot\left(h c N_{\mathrm{A}} / \lambda-\Delta_{\text {dis }} H\right) \mathrm{zu} \sim 150 \mathrm{~kJ} / \mathrm{mol}$ abgeschätzt werden. Dagegen steht eine Dissoziationsenthalpie von $19.4 \mathrm{~kJ} / \mathrm{mol}$ für die Abspaltung des endständigen Iodatoms aus dem Isomer [59], das Radikal muss demnach $90 \%$ seiner Energie an die Lösungsmittelumgebung abgeben, bevor das Isomer gebildet werden kann. Es erscheint sehr unwahrscheinlich, dass das Radikal innerhalb einer Picosekunde einen solch beträchtlichen Energiebetrag an das Lösungsmittel abgeben kann. Zudem sollte das einatomige Lösungsmittel Xenon viel ineffektiver Energie aufnehmen, die Ausbeute an Isomer für die schnelle Bildung und somit die Absorption innerhalb der ersten Picosekunde kleiner sein. Abb.6.1 zeigt zwei bei ähnlichen reduzierten Dichten aufgenommene und auf das Anfangsmaximum normierte Absorptionszeitprofile in $\mathrm{CO}_{2}$ und Xenon. Der Transient in Xenon zeigt um 1 ps keine signifikant geringere Absorption als das entsprechende Absorptionszeitprofil in $\mathrm{CO}_{2}$.

\subsection{3 $\mathrm{CH}_{2}$ I-Radikal}

Weil das $\mathrm{CH}_{2}$ I-Radikal direkt nach Photolyse schwingungsheiß ist, werden zur Abschätzung der Radikalabsorption heiße Absorptionsspektren benötigt. Bei 304 nm Anregung dissoziiert das Diiodmethanmolekül in das $\mathrm{CH}_{2}$ I-Radikal und einer Mischung aus $\sim 20 \% \mathrm{I}^{*}$ - sowie $\sim 80 \%$ I-Atomen (vergl. Abb.2.5). Für die folgende Betrachtung wird jedoch der Anteil an 
angeregten Iodatomen vernachlässigt. Zur Abschätzung der Extinktionskoeffizienten muss zunächst die Schwingungstemperatur des Radikals abgeschätzt werden, hierfür wiederum muss eine Annahme über die Verteilung der Überschussenergie auf die drei Rotations- und sechs Schwingungsfreiheitsgrade gemacht werden. Weil Baughcum und Leone Emission des Radikals über einen breiten Wellenlängenbereich detektiert haben [40], wird angenommen, dass die Überschussenergie gleichmäßig über alle Rotations- und Schwingungsfreiheitsgrade verteilt ist. Die Temperatur des Radikals kann nun gemäß

$$
\left\langle E_{\mathrm{CH}_{2} \mathrm{I}}\right\rangle=\sum_{i=1}^{6} \frac{h \nu_{i}}{\exp \left(\frac{h \nu_{i}}{k_{\mathrm{B}} T}\right)-1}+\frac{3}{2} k_{\mathrm{B}} T
$$

berechnet werden. Die Summe auf der rechten Seite ist der Schwingungsanteil zur inneren Energie $\left\langle E_{\mathrm{CH}_{2} \mathrm{I}}\right\rangle$, für den Rotationsanteil wird auf Grund der hohen Anregung der Hochtemperaturgrenzwert $3 / 2 k_{\mathrm{B}} T$ angenommen. Wird für die mittlere innere Energie $\left\langle E_{\mathrm{CH}_{2} \mathrm{I}}\right\rangle$ der Wert von $150 \mathrm{~kJ} / \mathrm{mol}$ aus dem vorhergehenden Abschnitt eingesetzt, ergibt sich mit aus DFT-Rechnungen stammenden Schwingungsfrequenzen [64] $\left(\nu_{1}=3174 \mathrm{~cm}^{-1}, \nu_{2}=1353 \mathrm{~cm}^{-1}\right.$, $\nu_{3}=609 \mathrm{~cm}^{-1}, \nu_{4}=166 \mathrm{~cm}^{-1}, \nu_{5}=3335 \mathrm{~cm}^{-1}, \nu_{6}=855 \mathrm{~cm}^{-1} ; k_{\mathrm{B}}$ und $h$ stellen die Boltzmannbzw. Planck'sche Konstante dar) eine Schwingungstemperatur $T_{\mathrm{v}} \simeq 3100 \mathrm{~K}$.

Wird die Schwingungstemperatur nach Kroger et al. [43] abgeschätzt, wonach sich nur $60 \%$ der Überschussenergie in den Schwingungs- und $40 \%$ in der Rotationsfreiheitsgraden befindet (vergl. Abschn.2.2.1) und wird der Energiebetrag von $\sim 90 \mathrm{~kJ} / \mathrm{mol}$ gleichmäßig auf alle Schwingungsfreiheitsgrade verteilt, führt dies zu einer Schwingungstemperatur von $T_{\mathrm{v}} \simeq 1800 \mathrm{~K}$.

In Abb.6.2 wurden die Absorptionsspektren des $\mathrm{CH}_{2}$ I-Radikals für 300, 1800 und $3100 \mathrm{~K}$ nach einem modifizierten Sulzer-Wieland Modell [127] abgeschätzt (siehe Anhang A.3). Um die Wellenlänge von $405 \mathrm{~nm}$ unterscheiden sich die beiden Hochtemperaturspektren kaum, d.h. die Art der Abschätzung der Schwingungstemperatur gibt in diesem Bereich keinen signifikanten Einfluss auf den Beitrag der Radikalabsorption. Bei der Wellenlänge von $405 \mathrm{~nm}$ ergibt bei sowohl 1800 als auch $3100 \mathrm{~K}$ ein Absorptionsquerschnitt von $\sim 0.55 \cdot 10^{-18} \mathrm{~cm}^{2} /$ molecule. Weil der Durchmesser des Pumpfokuses am Ort des Überlapps mit dem Probestrahl nicht bekannt ist, kann jedoch keine Absorptionsänderung angegeben werden.

Ein Argument gegen einen signifikanten Beitrag des Radikals zur Absorption ist die Ähnlichkeit der Absorptionszeitprofile von $\mathrm{CH}_{2} \mathrm{I}_{2}$ und $\mathrm{CH}_{2} \mathrm{BrI}$ (vergleiche Abb.4.2 und 4.24). Die Photolyse des $\mathrm{CH}_{2} \mathrm{BrI}$ wurde mit einer Anregungswellenlänge von $288 \mathrm{~nm}$ durchgeführt. Dabei wird selektiv die $\mathrm{C}-\mathrm{I}$-Bindung gebrochen und das $\mathrm{CH}_{2} \mathrm{Br}$-Radikal gebildet (siehe Abschn.2.3.1), welches jedoche keine Absorption bei Wellenlängen $>260 \mathrm{~nm}$ aufweist [53] (siehe auch Abb.2.6).

Als weiteres Argument gegen einen signifikanten Beitrag des Radikals zur Kurzzeitabsorption ist die Dichteabhängigkeit des Minimums: Wird angenommen, dass direkt nach Photolyse das $\mathrm{CH}_{2}$ I-Radikal mit einer Quantenausbeute von eins gebildet wird und dieser Wert unabhängig von der Dichte ist, so sollte das normierte Anfangsminimum nicht, wie in Abb.4.12 zu sehen, mit zunehmender Dichte ansteigen. 


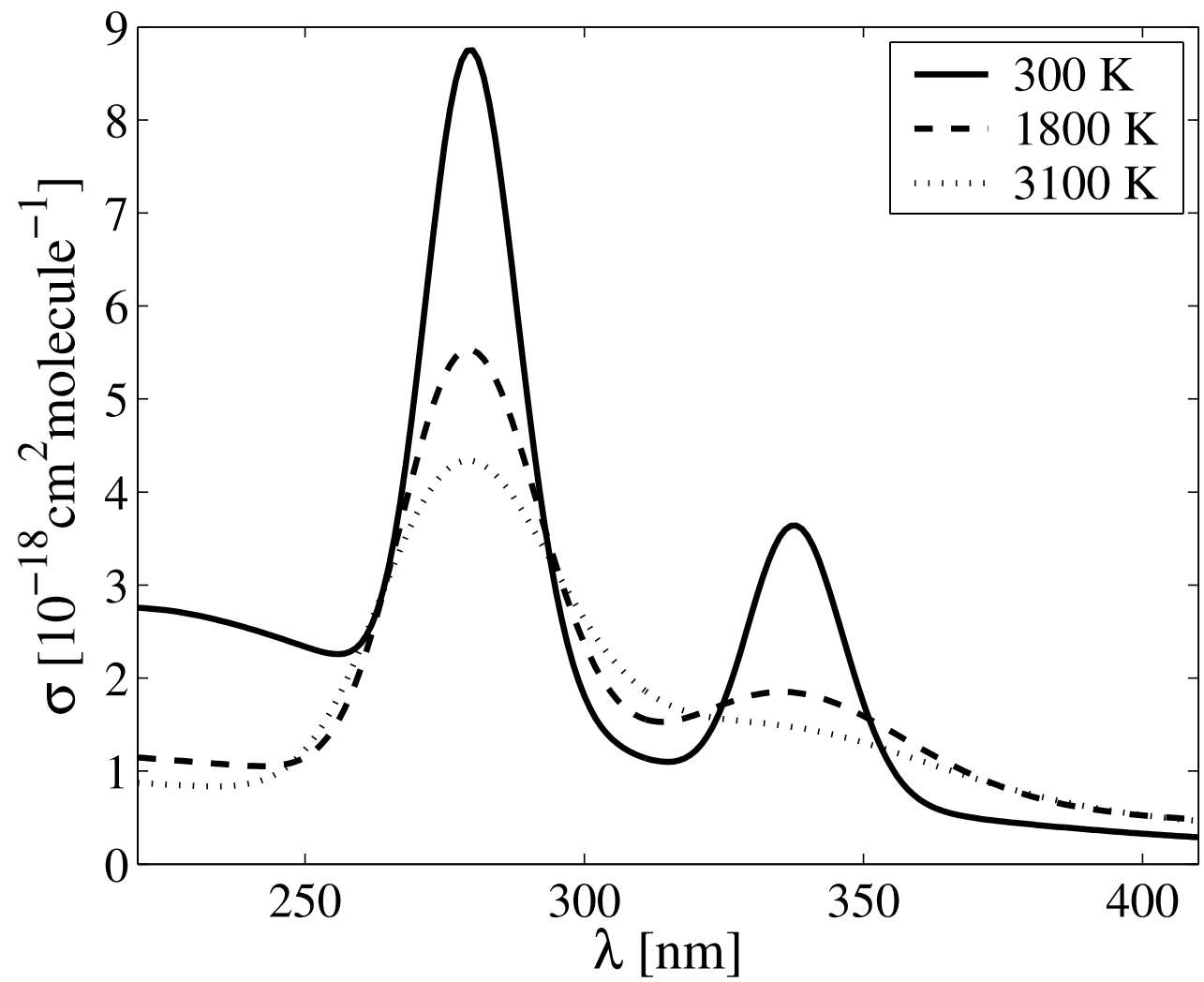

Abbildung 6.2: Simulation der Hochtemperaturspektren des $\mathrm{CH}_{2}$ I-Radikals nach [127].

\subsubsection{Geminale Rekombination zum Muttermolekül}

Ebenfalls wie bei der direkten Isomerbildung sprechen energetische Argumente gegen eine schnelle Rekombination zum Muttermolekül: Die $150 \mathrm{~kJ} / \mathrm{mol}$ Überschussenergie in Form von Schwingungsenergie müssen vor Rekombination dissipiert werden. Desweiteren sprechen sterische Effekte gegen eine Rekombination zum Muttermolekül: Weil das $\mathrm{CH}_{2}$ I-Radikal nach Dissoziation hoch rotationsangeregt ist, stellt sich nach sehr kurzer Zeit eine für die Rekombination ungünstige Geometrie ein. Die Ausbildung einer I - I-Bindung erscheint auf Grund der Größe des I- im Vergleich zum C-Atom wesentlich wahrscheinlicher.

Aufschluss über einen möglichen Beitrag der Rekombination zum Muttermolekül müsste ein Ausbleichexperiment mit einer Probewellenlänge in der Absorptionsbande des Muttermoleküls (um $300 \mathrm{~nm}$ ) geben. In einem solchen Experiment wird ein Ausbleichen der Absorption nach Photolyse innerhalb der Zeitauflösung erwartet sowie ein anschließender Anstieg auf Grund der Rekombination zum Muttermolekül. Rekombiniertes Diiodmethan wird schwingungsheiß vorliegen. Aus Stoßwellenuntersuchungen ist bekannt, dass die Absorption von schwingungsheißem $\mathrm{CH}_{2} \mathrm{I}_{2}$ bei $300 \mathrm{~nm}$ geringer als bei Raumtemperatur ausfällt [128]. In dem Ausbleichexperiment müsste demnach ein weiterer Anstieg der Absorption, hervorgerufen durch Schwingungskühlung, sichtbar sein. Experimentell konnte ein solches Verhalten jedoch nicht beobachtet werden [61]. 


\subsubsection{Ladungstransferkomplex}

Eine von uns vorgeschlagene Spezies [88, die für die Absorption im Kurzzeitbereich verantwortlich sein kann, ist ein Ladungstransferkomplex (charge transfer, CT-Komplex) aus Iodatom und $\mathrm{CH}_{2}$ I-Radikal. Es ist bekannt, dass CT-Komplexe aus Iodatomen als Elektronenakzeptor mit Alkyliodiden als Elektronendonor auftreten [129, 130. . Unsere Annahme ist, dass, begünstigt durch die räumliche Nähe der Fragmente im Lösungsmittelkäfig, ein solcher Komplex auch mit einem $\mathrm{CH}_{2}$ I-Radikal als Donor entsteht. Die aus der Literatur bekannten Ladungstransferkomplexe zeichnen sich in der Gasphase durch eine starke Absorption in der Größenordnung $10^{4} \mathrm{l} /(\mathrm{mol} \cdot \mathrm{cm})$ im Wellenlängenbereich von 300-400 $\mathrm{nm}$ aus. Die Frequenz $\nu_{\mathrm{CT}}$ des CT-Übergangs ergibt sich gemäß $h \nu_{\mathrm{CT}}=I_{\mathrm{D}}-E A_{\mathrm{A}}-e^{2} / d_{12}$, wobei $I_{\mathrm{D}}$ und $E A_{\mathrm{A}}$ das Ionisationspotential bzw. die Elektronenaffinität des Donors und Akzeptors, e die Elementarladung und $d_{12}$ der Gleichgewichtsabstand ist [129]. In Lösung hingegen verschiebt sich das Gleichgewicht zur Eduktseite, die Absorption weist höhere Extinktionskoeffizienten auf und das Spektrum wird rotverschoben [130].

\subsubsection{Orientierungsrelaxation}

Zur weiteren Charakterisierung der zu frühen Verzögerungszeiten absorbierenden Spezies wurden dichteabhängige Anisotropiemessungen durchgeführt. Wird die Anisotropie von nur einer Spezies verursacht, so wird ein zu den drei Rotationsachsen korrespondierender dreifach exponentiell abfallender Verlauf erwartet, jedoch ist eine experimentelle Beobachtung der drei Komponenten im Allgemeinen nicht möglich [110. Weil in dieser Arbeit nur eine einfach exponentiell abfallende Anisotropie beobachtet wird, wird davon ausgegangen, dass die Rotation um die Achse mit dem größten Trägheitsmoment die Orientierungsrelaxation verursacht.

Die Orientierungsrelaxation (oder auch Rotationsdiffusion) wird mit Hilfe des StokesDebye-Einstein Modells [110, 131] beschrieben:

$$
\tau_{\text {rot }}=\tau_{0}+\frac{\eta V}{k_{\mathrm{B}} T} f_{\text {stick }} C .
$$

$V$ ist das Volumen des Gelösten, $\eta$ die Lösungsmittelviskosität, $k_{\mathrm{B}}$ und $T$ sind die Boltzmannkonstante sowie die Temperatur, $f_{\text {stick }}$ ist ein molekularer Formfaktor, $C$ gibt die Kopplung von Gelösten und Lösungsmittel an und $\tau_{0}$ ist die freie Rotorzeit. Die Lebensdauer der

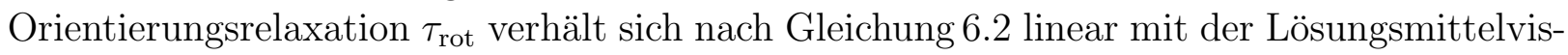
kosität. Weil die Messungen bei zwei verschiedenen Temperaturen durchgeführt wurden (35 und $50^{\circ} \mathrm{C}$ ), wurden in Abb. 6.3 die Lebensdauern $\tau_{\text {rot }}$ aus Abb. 4.28 gegen $\eta / T$ aufgetragen. Die Lösungsmittelviskositäten für $\mathrm{CO}_{2}$ wurden [116] entnommen. In der Auftragung zeigt sich kein Unterschied bei beiden verwendeten Probewellenlängen. Der Achsenabschnitt einer linearen Extrapolation ergibt gemäß Gl.6.2 $\tau_{0}$.

Zur Identifikation der Spezies, welche die Orientierungsrelaxation verursacht, werden die freien Rotorzeiten des Radikals, des Isomers und des Muttermoleküls berechnet: Für die freie Rotorzeit $\tau_{0}$ um eine Achse mit dem Trägheitsmoment $\Theta$ bei einer Temperatur $T$ gilt [132]:

$$
\tau_{0}=\sqrt{\frac{2 \pi \Theta}{9 k_{\mathrm{B}} T}} .
$$




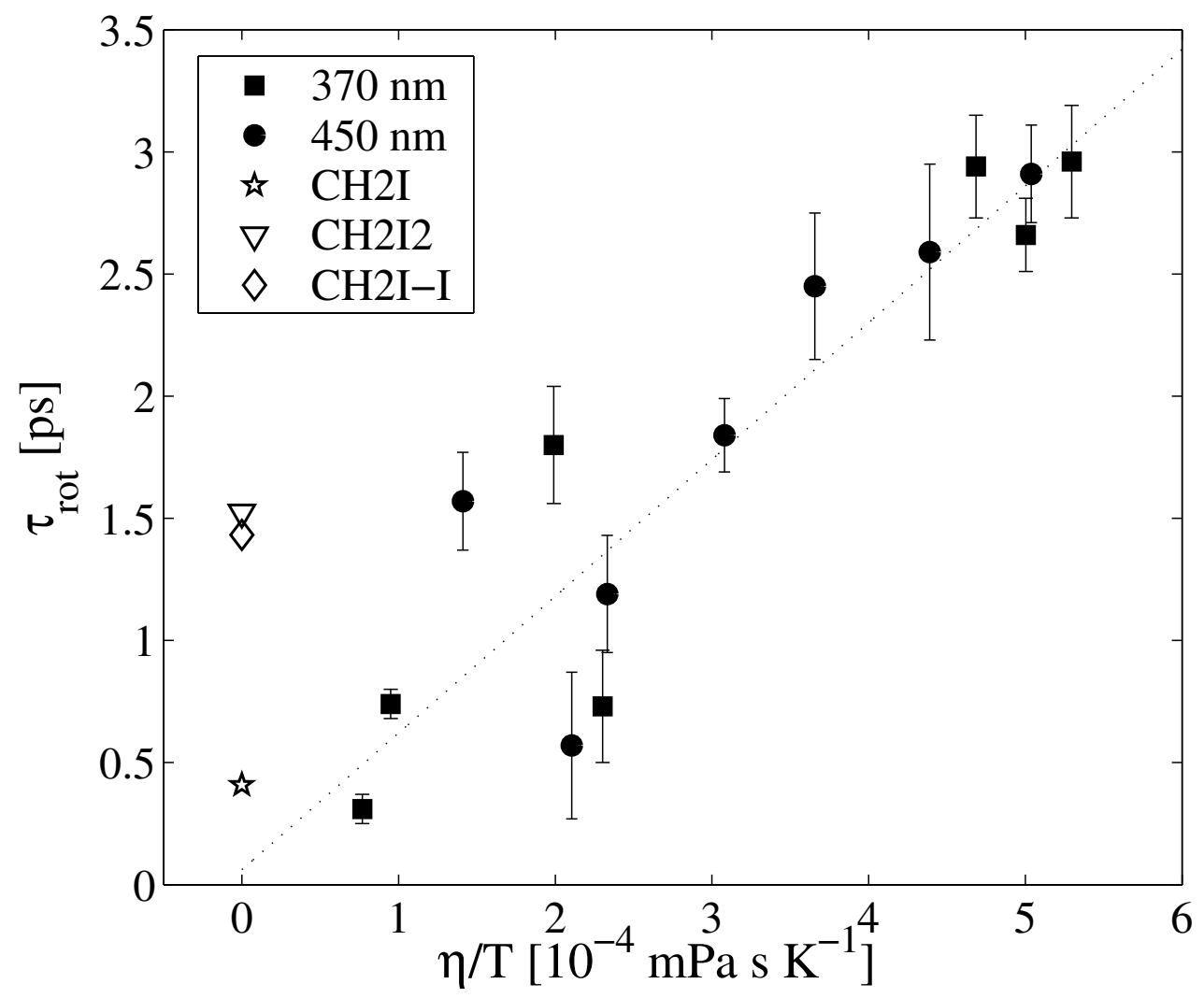

Abbildung 6.3: Auftragung der Orientierungsrelaxation $\tau_{\text {rot }}$ von $\mathrm{CH}_{2} \mathrm{I}_{2}$ gegen die Lösungsmittelviskosität von $\mathrm{CO}_{2}$ (geschlossene Symbole). Ebenfalls dargestellt sind die freien Rotorzeiten des Radikals, des Muttermoleküls und des Isomers (offene Symbole).

Die Trägheitsmomente des $\mathrm{CH}_{2}$ I-Radikals wurden [133] entnommen, die Trägheitsmomente des Muttermoleküls wurden aus den Rotationskonstanten A [134] gemäß $\Theta=h / 8 \pi^{2} A$ erhalten, während die des Isomers aus den berechneten Geometrien von Phillips et al. 64] durch die Beziehung $\Theta=\sum_{i} m_{i} r_{i}^{2}$ berechnet wurden. Die Trägheitsmomente aller drei Spezies sind in Tabelle6.1 zusammengefasst.

Es stellt sich nun die Frage nach der Rotationstemperatur in Gl.6.3. Aus MD-Simulationen ist bekannt, dass Rotationsfreiheitsgrade schnell innerhalb weniger Stöße mit Lösungsmittelmolekülen abkühlen [135. Die Abschätzung der Stoßzahl zwischen $\mathrm{CH}_{2} \mathrm{I}_{2}$ und $\mathrm{CO}_{2}$ erfolgt in Abschn.6.3.2. Das Ergebnis wird hier vorweggenommen: Bei einer Lösungsmitteldichte von $10 \mathrm{~mol} / \mathrm{l}$ beträgt die Stoßzahl $0.33 \mathrm{ps}^{-1}$, bei $28 \mathrm{~mol} / 10.14 \mathrm{ps}^{-1}$. Weil diese Stoßzahl klein im

\begin{tabular}{c|c|c}
$\mathrm{CH}_{2} \mathrm{I} / \mathrm{kg} \cdot \mathrm{m}^{2}$ & $\mathrm{CH}_{2} \mathrm{I}_{2} / \mathrm{kg} \cdot \mathrm{m}^{2}$ & $\mathrm{CH}_{2} \mathrm{I}-\mathrm{I} / \mathrm{kg} \cdot \mathrm{m}^{2}$ \\
\hline $9.793 \cdot 10^{-46}$ & $1.385 \cdot 10^{-44}$ & $1.209 \cdot 10^{-44}$ \\
$9.495 \cdot 10^{-46}$ & $1.352 \cdot 10^{-44}$ & \\
$3.011 \cdot 10^{-47}$ & $3.809 \cdot 10^{-46}$ &
\end{tabular}

Tabelle 6.1: Trägheitsmomente von $\mathrm{CH}_{2} \mathrm{I}, \mathrm{CH}_{2} \mathrm{I}_{2}$ und $\mathrm{CH}_{2} \mathrm{I}-\mathrm{I}$. 
Vergleich zur gemessenen Lebensdauer der Orientierungsrelaxation ist, wird angenommen, dass durch die Anisotropieexperimente thermisch äquilibrierte Rotation beobachtet wird. Demgemäß wird für die Rotationstemperatur die Lösungsmitteltemperatur (308 K) eingesetzt. Mit den jeweils größten Trägheitsmomenten aus Tabelle6.1 ergeben sich für die freien Rotorzeiten 0.40, 1.51 bzw. 1.41 ps für das Radikal, Muttermolekül bzw. Isomer. Vergleicht man nun diese freien Rotorzeiten mit der Extrapolation aus Abb.6.3, so kommt als Anisotropie verursachende Spezies nur das Radikal in Frage (der $\tau_{0}$-Wert liegt innerhalb der Fehlerbalken der Extrapolation). Jedoch wurde ein signifikanter Beitrag des Radikals schon in den vorhergehenden Abschnitten ausgeschlossen. Es erscheint wahrscheinlicher, dass sich das Übergangsdipolmoment des schwach gebundenen CT-Komplexes durch die Rotation des Radikals ändert.

\subsubsection{Zusammenfassung}

Das Maximum am Anfang der Absorptionszeitprofile kann durch die Absorption aus dem ersten elektronisch angeregten Zustand erklärt werden. Die Dichteabhängigkeit des der Dissoziation zugeschriebenen Abfalls konnte nicht aufgelöst werden. Die plausibelste Zuordnung für die nachfolgende Minimumsabsorption ist der von uns vorgeschlagene CT-Komplex als Hauptbestandteil. Die dichteabhängigen Anisotropiemessungen können in Übereinstimmung mit dieser Spezies interpretiert werden. Als weitere, jedoch wesentlich geringere Beiträge sind die des $\mathrm{CH}_{2}$ I-Radikals und möglicherweise die $S_{\mathrm{n}} \longleftarrow S_{1}$-Absorption des Muttermoleküls zu nennen.

\subsection{Quantenausbeuten}

Unter der Annahme, dass zu langen Verzögerungszeiten (> $50 \mathrm{ps}$ ) nur das Isomer zur Absorption beiträgt, ist die konstante Endabsorption direkt proportional zur Quantenausbeute an Isomer $\Phi_{\text {iso }}$. Wie in Abschn.4.2.2 ausführlich diskutiert, spiegelt sich die Dichteabhängigkeit der Quantenausbeute in der auf das Anfangsmaximum normierten Endamplitude $A_{\mathrm{E}, \mathrm{n}}$ wider: $A_{\mathrm{E}, \mathrm{n}} \propto \Phi_{\text {iso }}$. Für die Gesamtquantenausbeute gilt: $\Phi_{\text {dis }}+\Phi_{\text {iso }}+\Phi_{\text {rek }}=1$, wobei $\Phi_{\text {rek }}$ die Quantenausbeute für die geminale Rekombination zum Muttermolekül darstellt. Weil der spektroskopische Beweis für die Existenz dieses Reaktionskanals gegenwärtig nicht gegeben ist, vereinfacht sich die Gleichung zu $\Phi_{\text {dis }}+\Phi_{\text {iso }}=1$. Über diese Beziehung lassen sich die Quantenausbeuten für die Isomerbildung des Diiodmethans mit den Dissoziationsquantenausbeuten des Iods [35] vergleichen.

\subsubsection{Lösungsmittelabhängigkeit}

Aus den Untersuchungen zur Photodissoziationsquantenausbeute des Iods in überkritischen Lösungsmitteln ist bekannt, dass sich die in verschiedenen Fluiden bestimmten Quantenausbeuten eine übereinstimmende Abhängigkeit von der reduzierten Dichte aufweisen [35, 36] (siehe auch Abschn.2.1.3).

Daher werden in Abb. 6.4 die Amplituden $A_{\mathrm{E}, \mathrm{n}}$ aus Abb. 4.18 gegen die reduzierte Dichte aufgetragen. Diese Amplituden für die in Xenon, Kohlendioxid und Ethan durchgeführten 
Messungen liegen bis auf einige geringfügige Abweichungen aufeinander. Die Amplituden in $\mathrm{CHF}_{3}$ hingegen liegen signifikant über denen der anderen Lösungsmittel. Erklärbar ist dies dadurch, dass das Anfangsmaximum der Transienten in $\mathrm{CHF}_{3}$ wesentlich geringer als in den anderen Lösungsmitteln ausgeprägt ist (siehe Abb. A.9) und die Normierung auf dieses Maximum dementsprechend zu größeren Werten führt. Verursacht wird die Verringerung der Anfangsabsorption wahrscheinlich durch eine Lösungsmittelverschiebung des polaren Fluoroforms der $S_{\mathrm{n}} \longleftarrow S_{1}$-Absorptionsbande. Aus diesem Grund werden in Abb.6.5 zusätzlich die absoluten Amplituden $A_{\mathrm{E}}$ miteinander verglichen. Fluoroform und Kohlendioxid zeigen hier einen identischen Verlauf, während Xenon und Ethan wegen unterschiedlicher Anregungsintensitäten davon abweichen.

Die Quantenausbeuten für die Isomerbildung in den vier in dieser Arbeit verwendeten Lösungsmitteln lassen sich bis auf einige geringfügige Abweichungen befriedigend durch die reduzierte Dichte vergleichen. Die reduzierte Dichte ist dabei ein Maß für die Packungsdichte des Lösungsmittels um das Diiodmethan.

\subsubsection{Dichteabhängigkeit}

Bei Betrachtung der Isomerisierungs- und damit der Dissoziationsquantenausbeuten in $\mathrm{CO}_{2}$ im Dichtebereich $0.6<\rho_{\mathrm{r}}<2.4$ ergibt sich nicht wie im Falle des Iods 35] eine lineare Dichteabhängigkeit. Der Kurvenverlauf der $A_{\mathrm{E}, \mathrm{n}}$ in Abb.4.10 weist vielmehr eine positive Krümmung auf. Die Isomerisierungsquantenausbeute des Diiodmethans scheint somit zwar mit der Packungsdichte des Lösungsmittelkäfigs zusammenzuhängen, ist aber nicht mit einem einfachen kinematischen Modell (siehe Abschn.2.1.3) vollständig erklärbar.

Abb.6.6 zeigt die $A_{\mathrm{E}, \mathrm{n}}$ in Kohlendioxid bei einer Anregungswellenlänge von $304 \mathrm{~nm}$ und einer Abfragewellenlänge von $370 \mathrm{~nm}$. Zusätzlich wurde das Quadrat der Dichte $\rho^{2}$ aufgetragen. Abb. 6.7 zeigt das entsprechende Bild bei der Abfragewellenlänge von $405 \mathrm{~nm}$. Es ergibt sich in beiden Auftragungen eine gute Übereinstimmung der Amplituden mit $\rho^{2} \mathrm{im}$ oberen Dichtebereich $\left(\rho_{\mathrm{r}}>1.5\right)$ und geringfügige Abweichungen zu größeren Werten im Dichtebereich $\rho_{\mathrm{r}}<1.5$.

Diese quadratische Dichteabhängigkeit von $\Phi_{\text {iso }}$ kann durch zwei konsekutiv ablaufende Käfigausbrüche erklärt werden (siehe kinetisches Modell in Abb.6.8):

1. Nach Photoanregung dissoziiert das Diiodmethan auf einer repulsiven Potentialfläche in ein $\mathrm{CH}_{2}$ I-Radikal und ein I-Atom. Die Fragmente besitzen bei $304 \mathrm{~nm}$ Anregung eine relative kinetische Energie von $\sim 30 \mathrm{~kJ} / \mathrm{mol}$. Nachdem die beiden Fragmente sich voneinander getrennt haben, entscheidet es sich, ob sie durch Stoß mit dem Lösungsmittelkäfig zurückreflektiert werden oder ob sie ihn durchbrechen können. Die Quantenausbeute für den Käfigeinfang in diesem primären Schritt des Mechanismus ist $\Phi_{\text {prim. }}$, die Geschwindigkeitskonstante des zugehörigen Käfigausbruchs ist $k_{\text {prim. }}$. Weil die sekundäre geminale Rekombination vernachlässigt werden kann [37, 38, 39], werden im Folgenden nur die Fragmente betrachtet, die im Käfig verbleiben. Durch Stöße mit Lösungsmittelmolekülen geben die Fragmente auf einer 100 fs-Zeitskala ihre kinetische und rotatorische Energie ab, die Geschwindigkeitskonstante für diesen Prozess ist $k_{1}$. 


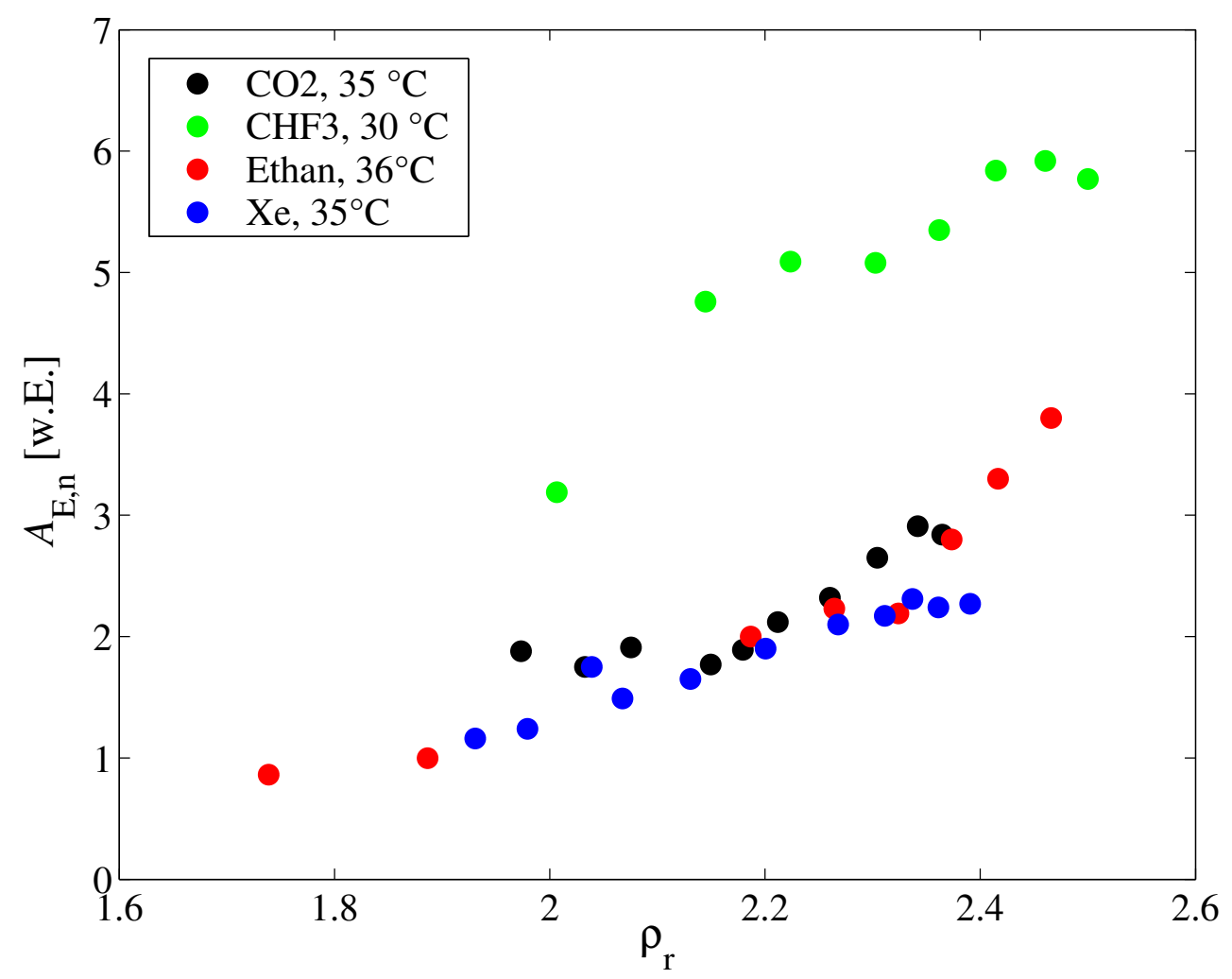

Abbildung 6.4: Auftragung der normierten Endamplituden $A_{\mathrm{E}, \mathrm{n}}$ gegen die reduzierte Lösungsmitteldichte von $\mathrm{CO}_{2}, \mathrm{C}_{2} \mathrm{H}_{6}, \mathrm{CHF}_{3}$ und Xe bei $405 \mathrm{~nm}$ Abfrage.

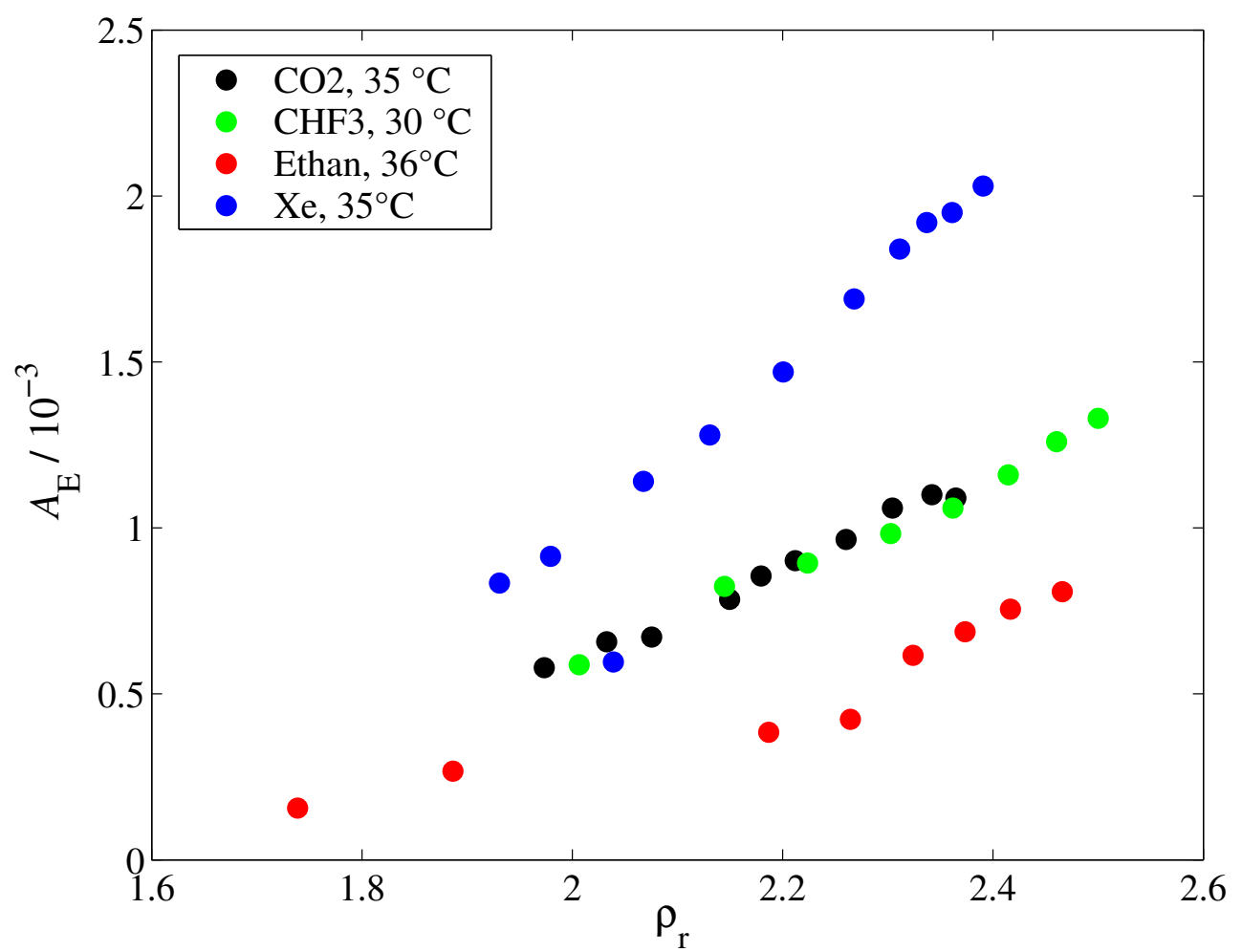

Abbildung 6.5: Auftragung der Endamplituden $A_{\mathrm{E}}$ gegen die reduzierte Lösungsmitteldichte von $\mathrm{CO}_{2}, \mathrm{C}_{2} \mathrm{H}_{6}, \mathrm{CHF}_{3}$ und Xe bei $405 \mathrm{~nm}$ Abfrage. 


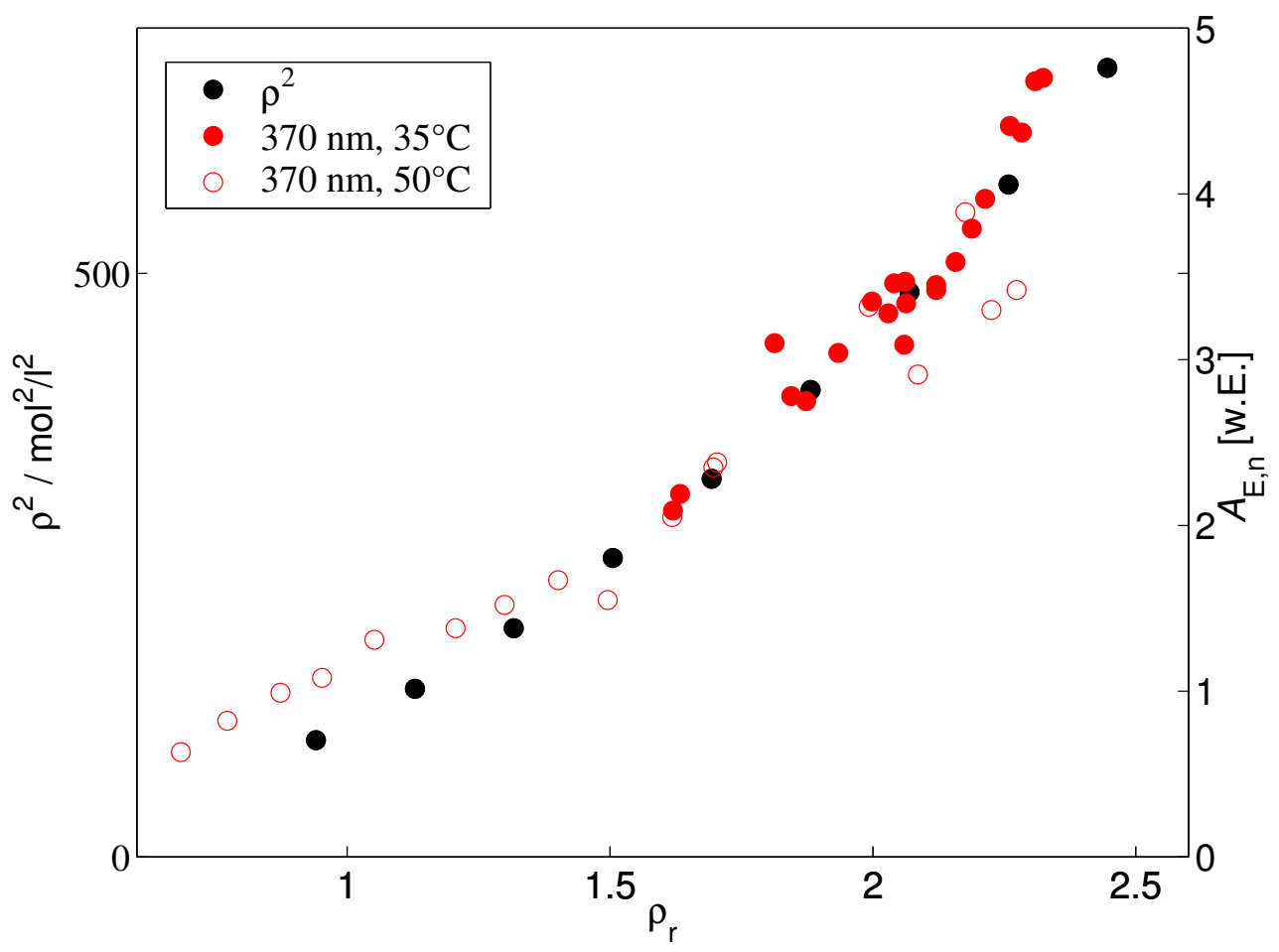

Abbildung 6.6: Korrelation der $A_{\mathrm{E}, \mathrm{n}}$ in $\mathrm{CO}_{2}$ bei $370 \mathrm{~nm}$ Abfrage mit $\rho^{2}$.

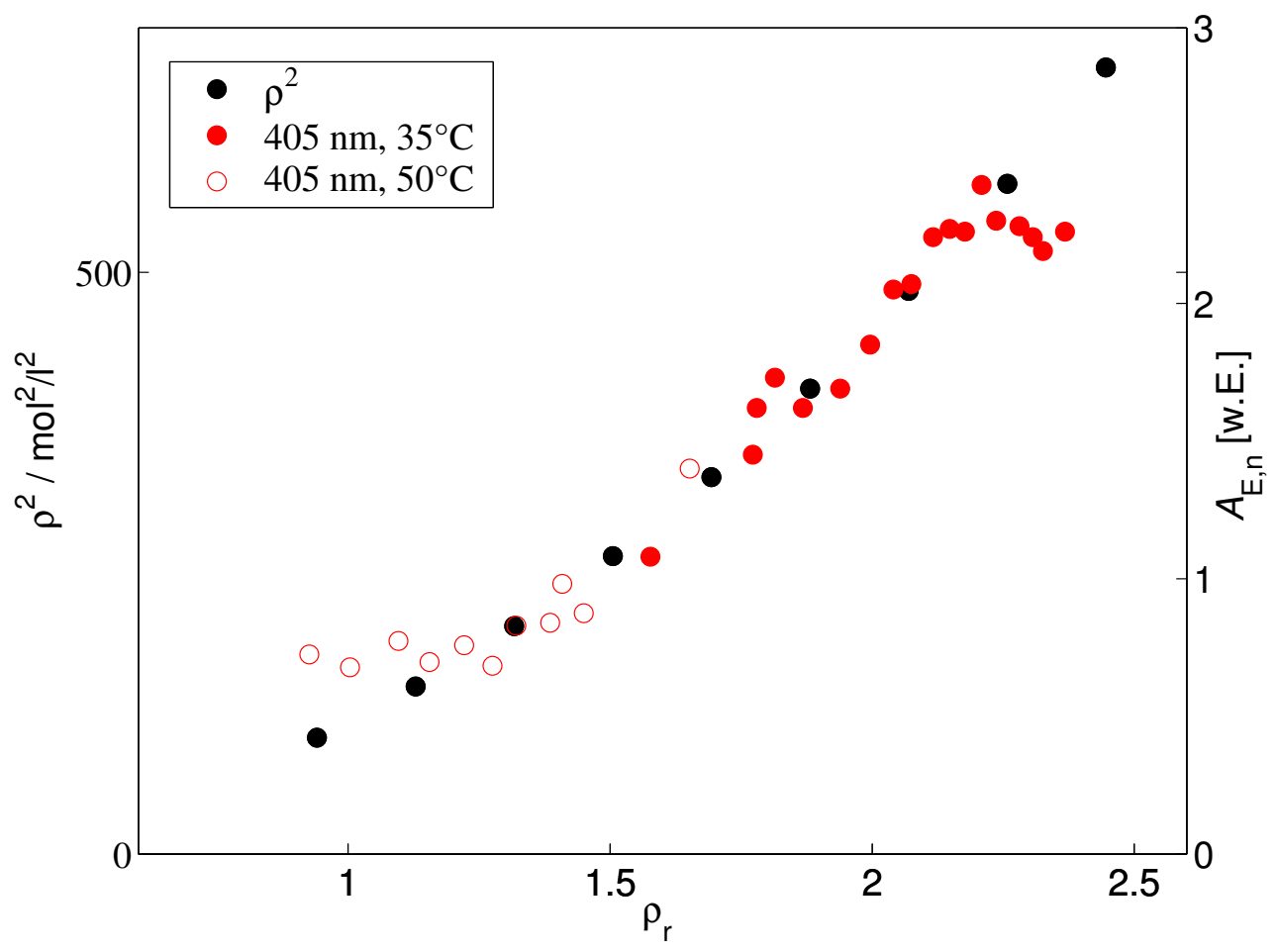

Abbildung 6.7: Korrelation der $A_{\mathrm{E}, \mathrm{n}}$ in $\mathrm{CO}_{2}$ bei $405 \mathrm{~nm}$ Abfrage mit $\rho^{2}$. 


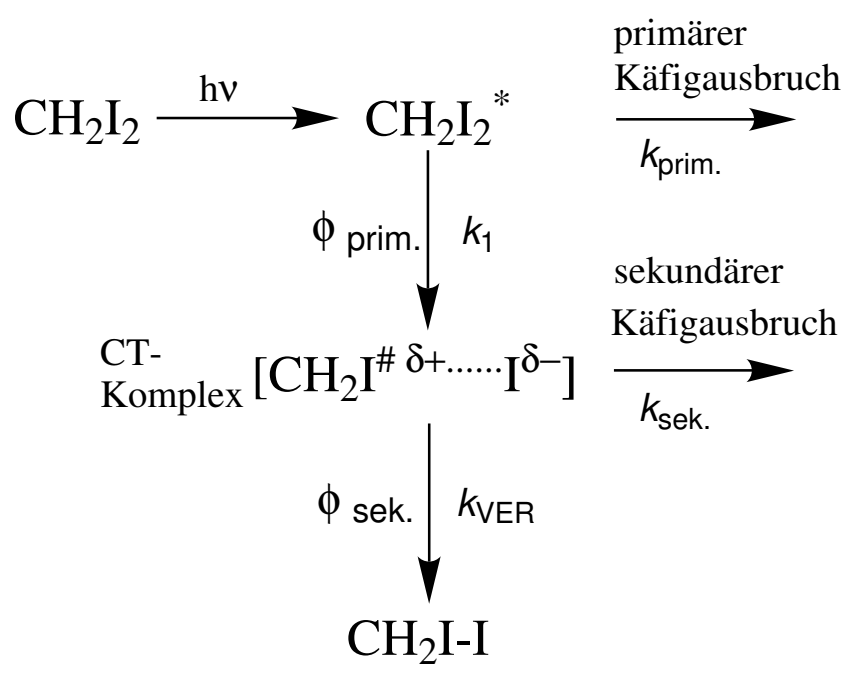

Abbildung 6.8: Vorläufiges kinetisches Modell.

2. Nachdem Translation und Rotation thermisch äquilibriert wurden, wird der in Abschn.6.1.5 vorgeschlagene CT-Komplex gebildet. Es wird erwartet, dass die Schwingungsenergierelaxation (mit der Geschwindigkeitskonstanten $k_{\mathrm{VER}}$ ) dieses Komplexes auf einer ps-Zeitskala abläuft und damit wesentlich langsamer als Translations- und Rotationsenergietransfer ist: Gemessene VER-Zeiten nach Anregung des ersten Obertons der C-H-Streckschwingung $\left(2 \nu_{1}\right)$ fallen in überkritischem $\mathrm{CO}_{2}$ im Dichtebereich $0.7<\rho_{\mathrm{r}}<1.7$ von 150 auf 50 ps ab [136]. Weil das Radikal im CT-Komplex erst $90 \%$ seiner Überschussenergie abgeben muss, bevor das Isomer gebildet werden kann, verbleibt für die Fragmente genügend Zeit den Käfig diffusiv zu verlassen. Die Geschwindigkeitskonstante für diesen diffusiven Käfigausbruch ist $k_{\text {sek. }}$, die Quantenausbeute für den Käfigeinfang im sekundären Schritt ist $\Phi_{\text {sek. }}$.

Die Gesamtausbeute an Isomer ergibt sich nun aus den Quantenausbeuten des primären und sekundären Käfigeinfangs gemäß $\Phi_{\text {iso }}=\Phi_{\text {prim. }} \cdot \Phi_{\text {sek. }}$. Es stellt sich nun die Frage nach der Dichteabhängigkeit der Quantenausbeuten $\Phi_{\text {prim. }}$ und $\Phi_{\text {sek. }}$. Der primäre Schritt des Mechanismus, die Separation der Photofragmente und der anschließende Käfigeinfang oder -ausbruch vor Abgabe des Überschusses an kinetischer Energie, ist identisch mit dem kinematischen Käfigeffekt des Iodmoleküls [35] und damit proportional zur Dichte: $\Phi_{\text {prim. }} \propto \rho$. Wird nun angenommen, dass die Quantenausbeuten für den sekundären Käfigeinfang ebenfalls proportional zur Dichte verlaufen $\left(\Phi_{\text {sek. }} \propto \rho\right)$, kann mit dem hier vorgeschlagenem konsekutiv ablaufenden Käfigausbruch die quadratische Dichteabhängigkeit von $\Phi_{\text {iso }}$ erklärt werden.

Ein weiterer Hinweis auf die Existenz des konsekutiven Käfigausbruchs ist die Dichteabhängigkeit der normierten Minima (siehe Abb.4.12 und 4.19): Wie im vorherigen Abschnitt dargestellt, wird die Minimumsabsorption dem CT-Komplex aus $\mathrm{CH}_{2}$ I-Radikal und Iodatom zugeschrieben. Die Quantenausbeute an CT-Komplex zu diesen frühen Verzögerungszeiten ist nach dem hier vorgeschlagenem Modell proportional zu $\Phi_{\text {prim. }}$ und damit proportional zur Dichte. In Abb.6.9 sind die normierten Minima bei $370 \mathrm{~nm}$ Abfrage und $50^{\circ} \mathrm{C}$ (die Messreihe mit dem größten Signal zu Rausch Verhältnis) gegen die Dichte aufgetragen. 


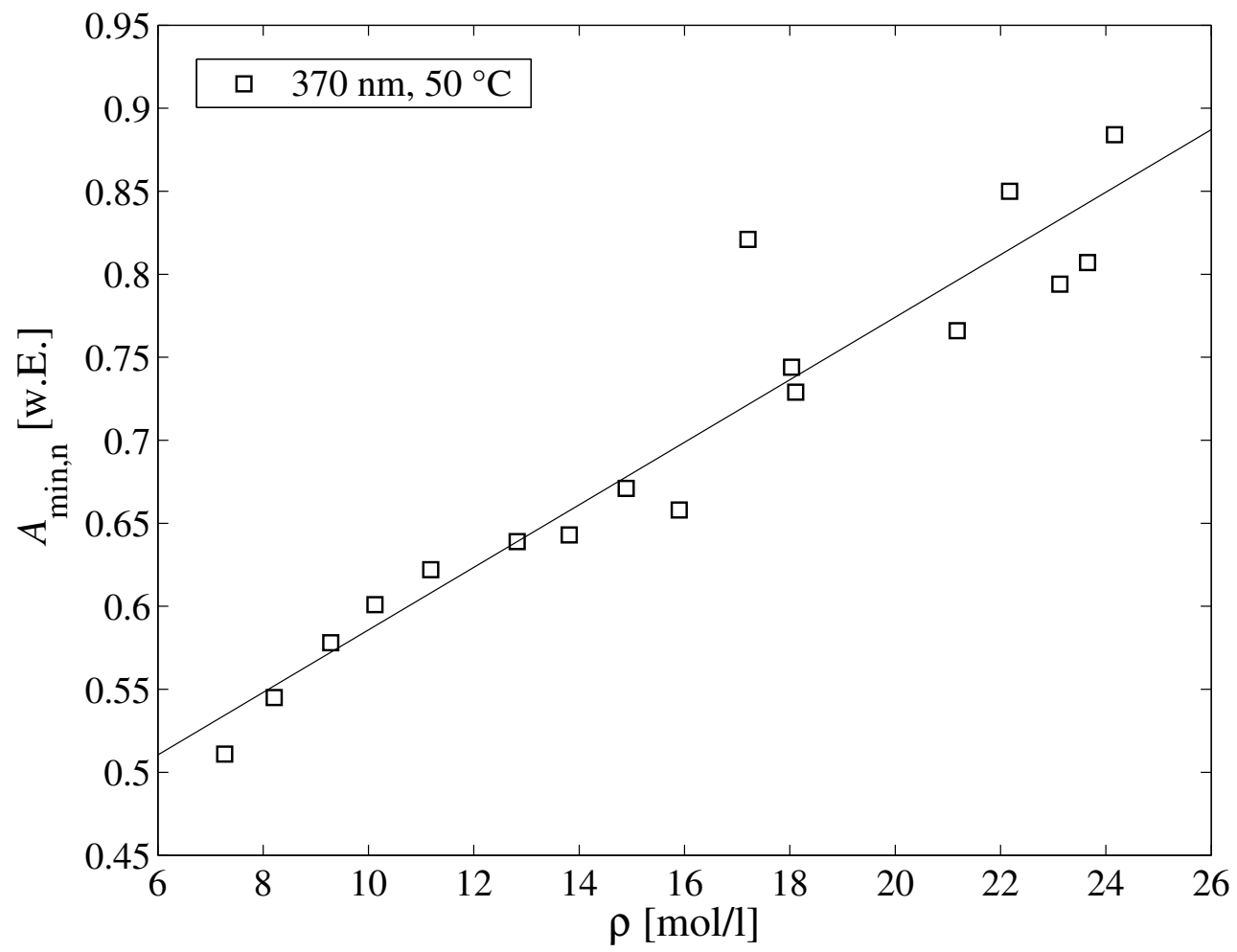

Abbildung 6.9: Auftragung des normierten Minimums $A_{\text {min,n }}$ gegen die Lösungsmitteldichte von $\mathrm{CO}_{2}\left(50^{\circ} \mathrm{C}\right)$ bei $304 \mathrm{~nm}$ Anregung und $370 \mathrm{~nm}$ Abfrage.

Die dichteabhängigen Minima lassen sich in Übereinstimmung mit dem Modell mit einer linearen Funktion anpassen. Diese Funktion verläuft jedoch nicht durch den Koordinatenursprung, sondern hat einen positiven Ordinatenabschnitt, was als als Hinweis verstanden werden kann, dass neben dem CT-Komplex noch weitere Spezies im Kurzzeitbereich zur Absorption beitragen.

Wie vorher beschrieben, weichen die $A_{\mathrm{E}, \mathrm{n}}$ im unteren Dichtebereich zu größeren Werten von $\rho^{2}$ ab. In Abb. 6.6 und 6.7 wurden die Endamplituden und damit die Quantenausbeuten an Isomer mit der homogenen Lösungsmitteldichte $\rho$ korreliert. Ausgehend von den MDSimulationen aus Kapitel 5 wird jedoch erwartet, dass die lokale Dichte um das Diiodmethan im unteren Dichtebereich von einer linearen Dichteabhängigkeit zu größeren lokalen Dichten abweicht. In den Abb.6.10 und 6.11 werden daher die Quantenausbeuten mit dem Quadrat der lokalen Dichte $g_{\max }^{2} \rho^{2}$ korreliert. Diese Korrelation impliziert die Annahme, dass sich die lokale Dichte im Zeitbereich zwischen Photoanregung und Isomerbildung nicht ändert, d.h. die Struktur der Lösungsmittelumgebung entspricht während der gesamten Dynamik der um das Muttermolekül. Obwohl die verwendeten Potentiale nicht für die überkritische Phase entwickelt wurden, stimmt das Quadrat der berechneten lokalen Dichten sehr gut mit den gemessenen $A_{\mathrm{E}, \mathrm{n}}$ überein. Der photolytische Käfigeffekt des Diiodmethans in überkritischem Kohlendioxid zeigt somit eindeutig eine lokale Dichtevergrößerung.

In Anhang A.5 sind die Korrelationen für die Lösungsmittel Xenon, Ethan und Fluoroform dargestellt. In Xenon (Abb.A.10) ist die Steigung der normierten Amplituden geringfügig 


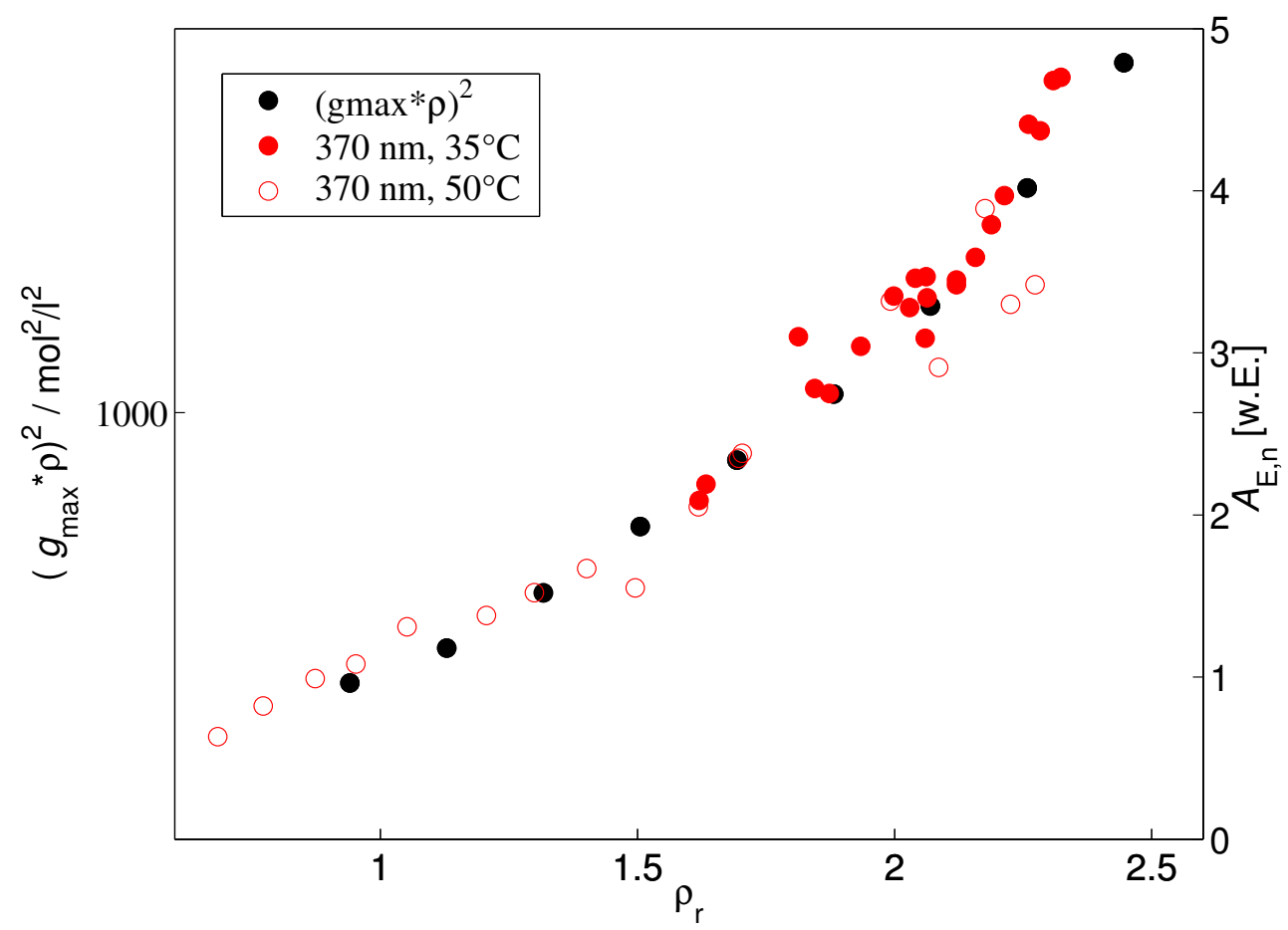

Abbildung 6.10: Korrelation der $A_{\mathrm{E}, \mathrm{n}}$ in $\mathrm{CO}_{2}$ bei $370 \mathrm{~nm}$ Abfrage mit $\left(g_{\max } \cdot \rho\right)^{2}$.

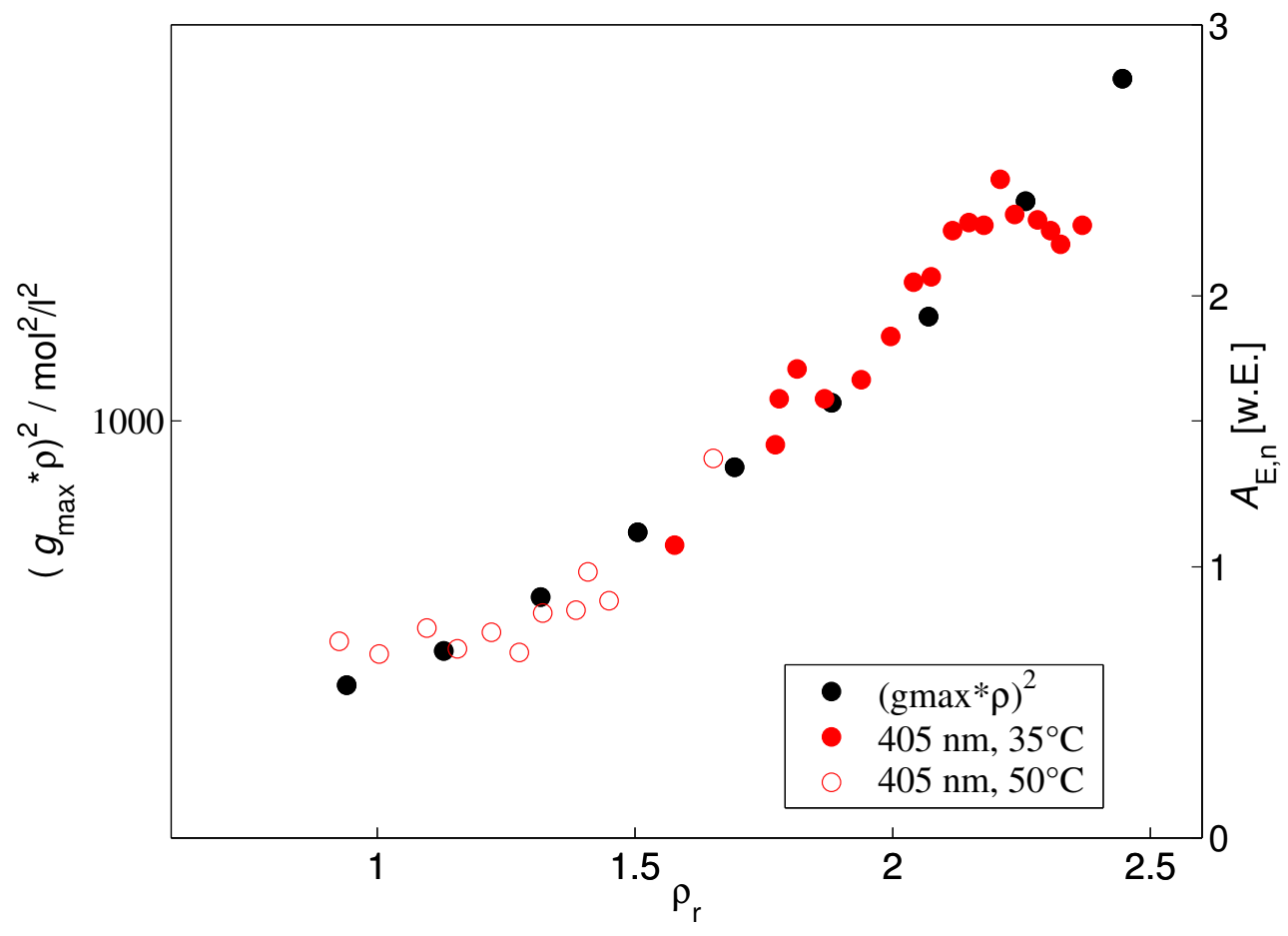

Abbildung 6.11: Korrelation der $A_{\mathrm{E}, \mathrm{n}}$ in $\mathrm{CO}_{2}$ bei $405 \mathrm{~nm}$ Abfrage mit $\left(g_{\max } \cdot \rho\right)^{2}$. 


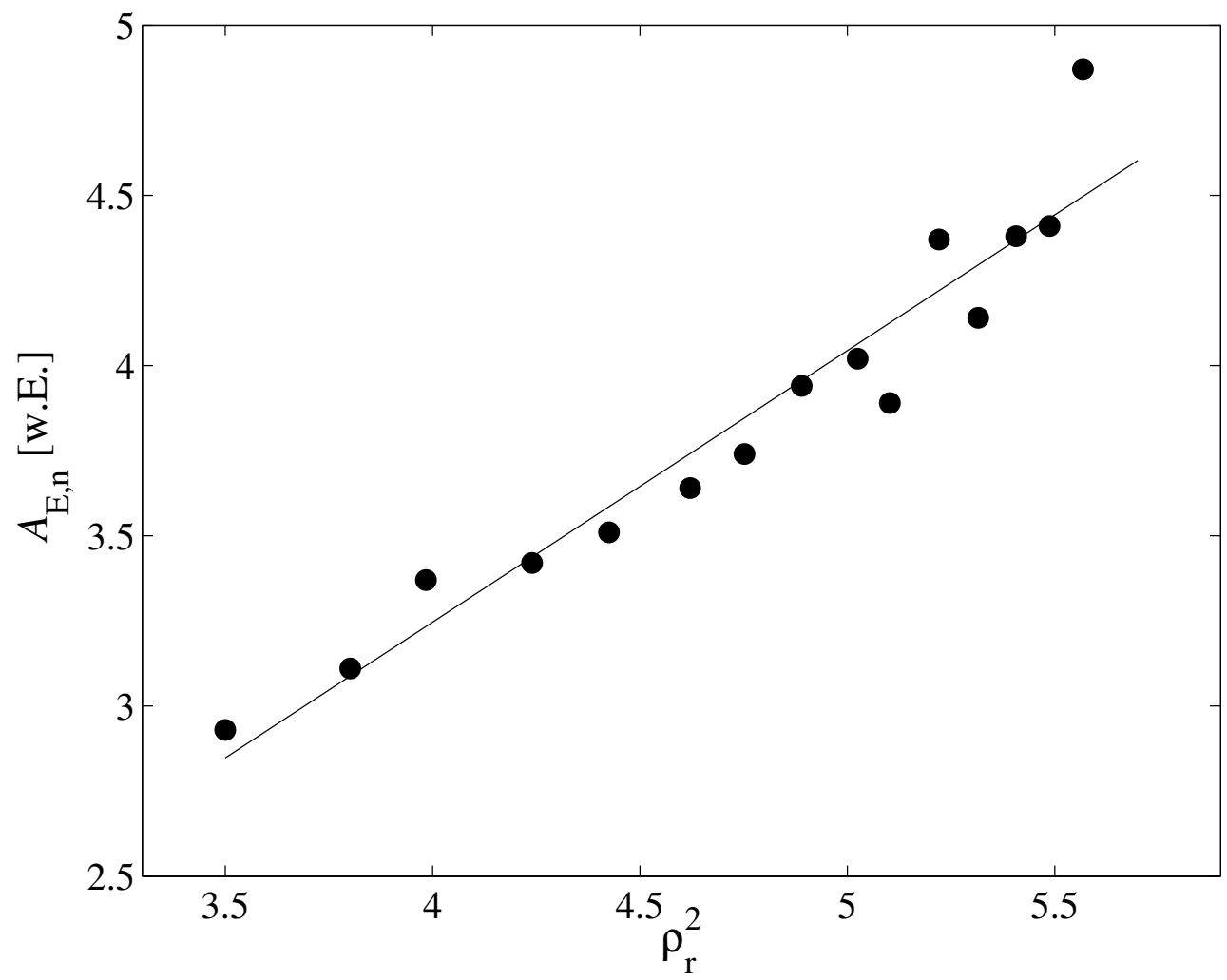

Abbildung 6.12: Auftragung der normierten Endamplitude $A_{\mathrm{E}, \mathrm{n}}$ gegen das Quadrat der reduzierten Dichte von $\mathrm{CH}_{2} \mathrm{BrI}$ in $\mathrm{CO}_{2}$ mit linearer Anpassung.

größer als die der entsprechenden lokalen Dichten. In Fluoroform (siehe Abb.A.11) passen Amplituden und lokale Dichte aufeinander, während in Ethan (siehe Abb.A.12) die Krümmung der Kurve durch das Modell unterschätzt wird. Das Versagen des Modells für Ethan ist $\mathrm{zu}$ diesem Zeitpunkt nicht verstanden.

Um zu überprüfen, ob bei den Isomerisierungsquantenausbeuten von $\mathrm{CH}_{2} \mathrm{BrI}$ in $\mathrm{CO}_{2}$ ebenfalls eine quadratische Dichtabhängigkeit vorliegt, wurden in Abb.6.12 die normierten Endamplituden aus Abb.4.25 gegen das Quadrat der reduzierten Dichte aufgetragen. Die Messpunkte lassen sich befriedigend in Übereinstimmung mit dem Modell des konsekutiven Käfigausbruchs linear anpassen.

An dieser Stelle sei noch einmal darauf hingewiesen, dass der Hauptunterschied des Käfigeffekts zwischen $\mathrm{I}_{2}$ und $\mathrm{CH}_{2} \mathrm{I}_{2}$ der ist, dass das Photofragment $\mathrm{CH}_{2} \mathrm{I}$ innere Freiheitsgrade besitzt. Diese inneren Freiheitsgrade sind nach Bildung des Fragments hoch angeregt. Die zur Isomerbildung nötige Schwingungsenergierelaxation auf einer ps-Zeitskala ermöglicht einen bei der Iodrekombination nicht auftretenden sekundären, diffusiven Käfigausbruch.

\subsection{Bildungsgeschwindigkeit}

Wie im vorherigem Abschnitt diskutiert, sind die Zeitskalen für den primären und sekundären Käfigausbruch separierbar: $k_{\mathrm{VER}}, k_{\text {sek. }} \ll k_{1}, k_{\text {prim. }}$. Der primäre Käfigeffekt hat somit 


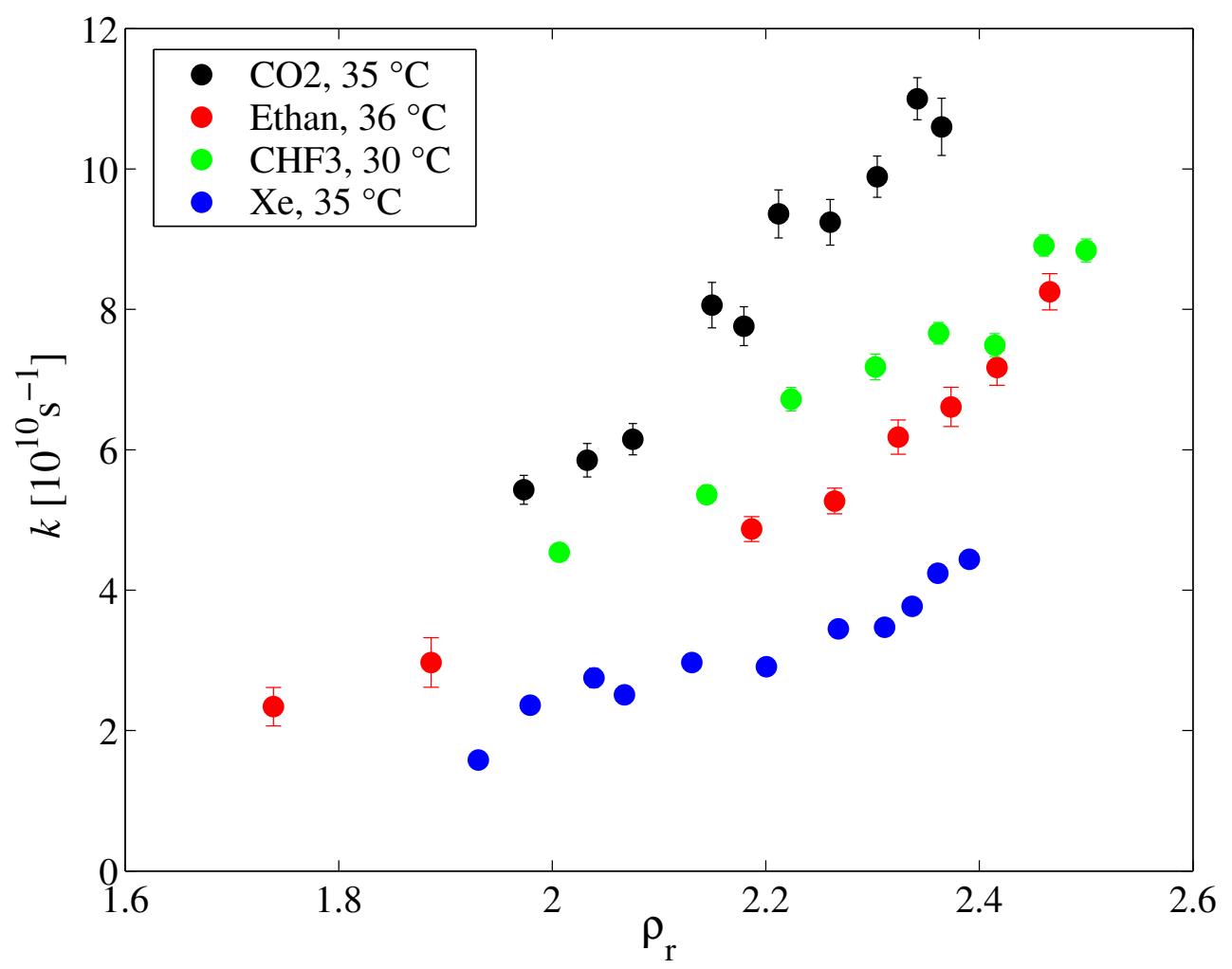

Abbildung 6.13: Auftragung von $k$ gegen die reduzierte Lösungsmitteldichte von $\mathrm{CO}_{2}, \mathrm{C}_{2} \mathrm{H}_{6}$, $\mathrm{CHF}_{3}$ und Xe bei $405 \mathrm{~nm}$ Abfrage.

keinen Einfluss auf die Isomerisierungsgeschwindigkeit $k$. Diese Bildungsgeschwindigkeit ergibt sich nach dem kinetischen Modell aus Abb.6.8 zu $k=k_{\mathrm{VER}}+k_{\text {sek. }}$. Wie bereits im Ergebnisteil dargestellt, kann $k$ mit der Anstiegszeit der Absorptionszeitprofile bei der Abfragewellenlänge von $405 \mathrm{~nm}$ identifiziert werden.

\subsubsection{Lösungsmittelabhängigkeit}

In Abb. 6.13 werden die Bildungsgeschwindigkeiten $k$ aus Abb.4.17 gegen die reduzierte Dichte aufgetragen. In dieser Auftragung zeigt sich eine Reihenfolge für die Isomerisierungsgeschwindigkeiten $k\left(\mathrm{CO}_{2}\right)>k\left(\mathrm{CHF}_{3}\right)>k\left(\mathrm{C}_{2} \mathrm{H}_{6}\right)>k(\mathrm{Xe})$. Weil sich nicht wie im Falle der Quantenausbeuten eine Übereinstimmung der verschiedenen Lösungsmittel ergibt, ist der Lösungsmitteleinfluss auf die Bildungsgeschwindigkeit nicht alleine durch die Packungsdichte erklärbar.

Das Lösungsmittel hat einen Einfluss auf beide Geschwindigkeitskoeffizienten, $k_{\text {VER }}$ und $k_{\text {sek. }}$. Größe, Masse und Struktur der Lösungsmittelmoleküle bestimmen über die Stoßzahl und die Stoßübergangswahrscheinlichkeit die Geschwindigkeit der Schwingungsenergierelaxation $k_{\text {VER }}$. Die Lösungsmittelviskosität hingegen kontrolliert die Mobilität der Teilchen und damit die Geschwindigkeit für den diffusiven Käfigausbruch $k_{\text {sek. }}$. Im Folgenden wird qualitativ beschrieben, wie beide Prozesse modelliert werden können. 
- $k_{\mathrm{VER}}$ : Nach Laseranregung wird eine mikrokanonische Energieverteilung mit der mittleren Energie $\langle E\rangle$ gebildet, welche durch Stöße mit Lösungsmittelmolekülen in die thermische Gleichgewichtsverteilung relaxiert. Wenn die innere Energie hinreichend weit vom thermischen Gleichgewicht entfernt ist, kann ihre zeitliche Entwicklung gemäß

$$
\frac{\mathrm{d}\langle E\rangle(t)}{\mathrm{d} t}=Z \cdot\langle\Delta E(\langle E\rangle)\rangle
$$

angegeben werden [137]. Dabei ist $Z$ eine energieunabhängige Stoßzahl und $\langle\Delta E(\langle E\rangle)\rangle$ die mittlere pro Stoß übertragene Energie. Hängt die mittlere pro Stoß übertragene Energie linear von der inneren Energie ab, fällt $\langle E\rangle(t)$ einfach exponentiell mit dem Geschwindigkeitskoeffizienten $k_{\text {VER }}$ ab [138. Das Lösungsmittel nimmt nach Gl.6.4 über die Stoßzahl und seine Fähigkeit, Schwingungsenergie aufzunehmen, Einfluss auf $k_{\mathrm{VER}}$.

- $k_{\text {sek. }}$ : Der Geschwindigkeitskoeffizient $k_{\text {sek. }}$ ist die reziproke Diffusionszeit $\tau_{\text {sek. }}$, die ein Teilchen benötigt, um die erste Lösungsmittelschale zu überwinden. $\tau_{\text {sek. }}$ kann gemäß

$$
\tau_{\text {sek. }}=\frac{\left\langle x^{2}\right\rangle}{2 D_{\mathrm{I}}}
$$

abgeschätzt werden. $\left\langle x^{2}\right\rangle$ ist die mittlere quadratische durch Diffusion zurückgelegte Strecke, wobei $\langle x\rangle$ mit dem Lennard-Jones Durchmesser des Lösungsmittelmoleküls gleichgesetzt werden kann. $D_{\text {I }}$ stellt den Diffusionskoeffiezienten des Iodatoms in dem betreffenden Lösungsmittel dar. In Gl.6.5 wurde der Diffusionskoeffizient des $\mathrm{CH}_{2} \mathrm{I}-$ Radikals mit dem des Iodatoms gleichgesetzt.

\subsubsection{Dichteabhängigkeit}

Im Folgenden wird versucht, die Dichteabhängigkeit der Bildungsgeschwindigkeit $k$ mit dem kinetischen Modell aus Abb.6.8 zu erklären. Die Bildungsgeschwindigkeit ergibt sich nach diesem Modell, wie an anderer Stelle schon bemerkt, gemäß $k=k_{\text {VER }}+k_{\text {sek. }}$. Für die Quantenausbeute des sekundären Käfigeinfangs gilt $\Phi_{\text {sek. }}=k_{\mathrm{VER}} /\left(k_{\mathrm{VER}}+k_{\text {sek. }}\right)$. Das Produkt aus Quantenausbeute und Bildungsgeschwindigkeit ergibt $k \cdot \Phi_{\text {sek. }}=k_{\mathrm{VER}}$.

Mit der Annahme $\Phi_{\text {sek. }} \propto \rho$ aus dem vorhergehendem Abschnitt und der Dichteabhängigkeit von $k$ aus Abb.4.14 wird das Produkt stark nichtlinear dichteabhängig. $k_{\text {VER }}$ wächst hingegen über die Stoßzahl aus G1.6.4 proportional mit der Dichte bzw. lokalen Dichte an: $k_{\text {VER }} \propto \rho$ [87,138,139]. Das Modell zur Erklärung der quadratischen Dichteabhängigkeit der Isomerisierungsquantenausbeute gibt folglich die Dichteabhängigkeit der Bildungsgeschwindigkeit nicht wieder.

In Abb.6.14 wird der vorläufige Reaktionsmechanismus um ein Vorgleichgewicht erweitert: Direkt nach dem primären Käfigeinfang stellt sich im Lösungsmittelkäfig ein Gleichgewicht zwischen unkomplexierten Photofragmenten und dem CT-Komplex ein. Für die Gleichgewichtskonstante gilt $K=c\left(\left[\mathrm{CH}_{2} \mathrm{I}^{\#++} \ldots \mathrm{I}^{\delta-}\right]\right) / c\left(\left[\mathrm{CH}_{2} \mathrm{I}^{\#}+\mathrm{I}\right]\right)=k_{2} / k_{-2}$, wobei $k_{2}$ und $k_{-2}$ die Geschwindigkeitskoeffizienten für die Hin- bzw. Rückreaktion sind. Unter der Annahme 


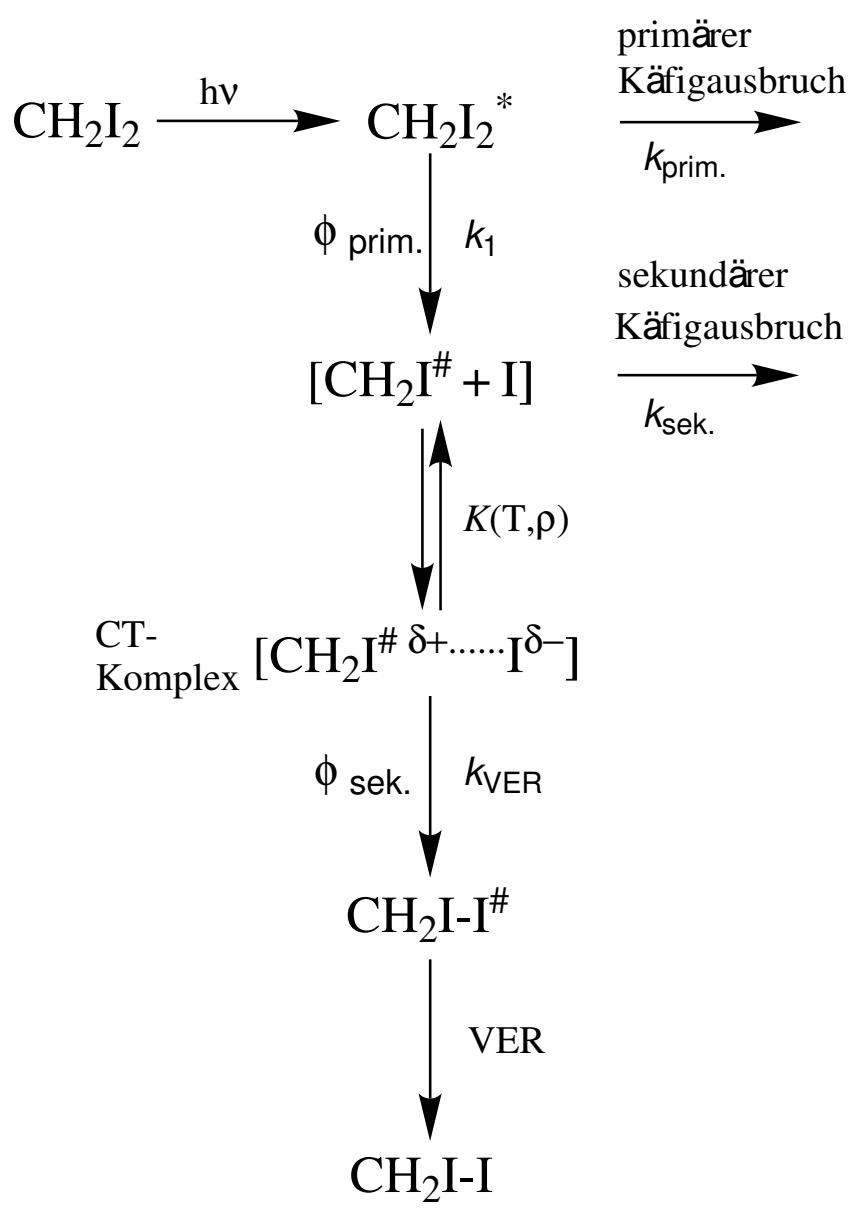

Abbildung 6.14: Kinetisches Modell.

der Quasistationarität des CT-Komplexes und mit $k_{2}, k_{-2} \gg k_{\text {sek. }}, k_{\mathrm{VER}}$ ergibt sich in diesem Modell die Bildungsgeschwindigkeit zu

$$
k=k_{\mathrm{sek} .}+K \cdot k_{\mathrm{VER}} .
$$

Für die Quantenausbeute des sekundären Käfigeinfangs gilt:

$$
\Phi_{\text {sek. }}=\frac{K \cdot k_{\mathrm{VER}}}{k_{\mathrm{sek} .}+K \cdot k_{\mathrm{VER}}} .
$$

Nach diesem Modell erhält man für das Produkt aus Bildungsgeschwindigkeit und Quantenausbeute $k \cdot \Phi_{\text {sek. }}=K \cdot k_{\mathrm{VER}}$. Die Dichteabhängigkeit der Bildungsgeschwindigkeit kann nun erklärt werden, sobald die Gleichgewichtskonstante $K$ dichteabhängig wird.

Im Folgenden wird die Dichteabhängigkeit von $K, k_{\mathrm{VER}}$ und $k_{\text {sek. }}$ diskutiert:

- $K$ : Aus der bekannten Beziehung $\Delta_{\mathrm{R}} G=-R T \ln K$ ergibt sich für die Druckabhängigkeit der Gleichgewichtskonstanten folgender Zusammenhang:

$$
\frac{\partial \ln K}{\partial p}=-\frac{1}{R T}\left(\frac{\partial \Delta_{\mathrm{R}} G^{\mathrm{id}}}{\partial p}+\frac{\partial \Delta_{\mathrm{R}} G^{\mathrm{solv}}}{\partial p}\right)
$$


Dabei wurde die freie Reaktionsenthalpie $\Delta_{\mathrm{R}} G$ in eine ideale Komponente $\Delta_{\mathrm{R}} G^{\text {id }}$ und einen Solvatationskomponente $\Delta_{\mathrm{R}} G^{\text {solv }}$ aufgeteilt. $\Delta_{\mathrm{R}} G^{\text {solv }}$ stellt hierbei die Änderung der Lösungsmittelstabilisierung zwischen Produkten und Edukten dar. Lösungsmittelstabilisierungen durch elektrostatische Wechselwirkungen werden überlicherweise durch das Onsager Modell beschrieben [140, 141, 142]. In diesem Modell wird das gelöste Teilchen durch einen Punktdipol $\mu$ im Mittelpunkt einer kugelförmigen Kavität mit dem Radius $r$ dargestellt. Die Kavität ist in einem polarisierbaren Dieelektrikum der Dieelektrizitätskonstante $\epsilon$ eingebettet. Der Punktdipol polarisiert das umgebende Dieelektrikum, was wiederum in der Kavität ein elektrisches Feld aufbaut. Die Energie des Punktdipols in diesem sogenannten Reaktionsfeld ist identisch mit der Lösungsmittelstabilisierung gegenüber dem Vakuum $\Delta G^{\text {solv,vak: }}$

$$
\Delta G^{\text {solv,vak }}=-N_{\mathrm{A}} \cdot \frac{\mu^{2}}{4 \pi \epsilon_{0} r^{3}} \frac{\epsilon-1}{2 \epsilon+1},
$$

mit der Avogadro- bzw. elektrischen Feldkonstanten $N_{\mathrm{A}}$ und $\epsilon_{0}$. Für die Änderung der Lösungsmittelstabilisierung gilt nun: $\Delta_{\mathrm{R}} G^{\text {solv }}=\Delta G^{\text {solv, vak }}$ (Produkte) $\Delta G^{\text {solv,vak }}$ (Edukte). Unter der Annahme, dass auf der Zeitskala der Reaktion nur die elektronische Polarisierbarkeit zur Stabilisierung beiträgt, ergibt sich mit der Maxwell Beziehung $n^{2}=\epsilon$ :

$$
\Delta_{\mathrm{R}} G^{\text {solv }}=-N_{\mathrm{A}} \cdot \frac{\left.\mu^{2} \text { (Produkte }\right)-\mu^{2}(\text { Edukte })}{4 \pi \epsilon_{0} r^{3}} \frac{n^{2}-1}{2 n^{2}+1},
$$

wobei $n$ den Brechungsindex darstellt.

Mit der Ableitung der idealen Komponente nach dem Druck $\left(\partial \Delta_{\mathrm{R}} G^{\text {id }}\right) /(\partial p)=\Delta_{R} V$ $\left(\Delta_{R} V\right.$ : Volumenänderung) ergibt sich für die Druckabhängigkeit der Gleichgewichtskonstanten:

$$
\frac{\partial \ln K}{\partial p}=-\frac{\Delta_{\mathrm{R}} V}{R T}+\frac{N_{\mathrm{A}}}{R T} \cdot \frac{\mu^{2}(\text { Produkte })-\mu^{2}(\text { Edukte })}{4 \pi \epsilon_{0} r^{3}} \frac{\partial \frac{n^{2}-1}{2 n^{2}+1}}{\partial p} .
$$

Die Dichteabhängigkeit des Brechungsindex für die Lösungsmittel Kohlendioxid, Ethan und Fluoroform ist z. B. von Maroncelli et al. [143] gegeben. Weil $\Delta_{\mathrm{R}} V$ für die Komplexbildung negativ ist und angenommen werden kann, dass das Dipolmoment des CT-Komplexes größer als das der nicht komplexierten Käfigfragmente ist, werden beide Summanden auf der rechten Seite positiv. Die Gleichgwichtskonstante $K$ wird nach dieser qualitativen Betrachtung mit zunehmender Dichte größer. Als weitere Näherung wird angenommen, dass das Volumen des umkomplexierten Käfigpaares und des CT-Komplexes nahezu identisch sind, d. h. die Dichteabhängigkeit von $K$ reduziert sich auf die Dichteabhängigkeit des Clausius-Mosotti Faktors

$$
\frac{\partial \ln K}{\partial \rho} \approx \frac{N_{\mathrm{A}}}{R T} \cdot \frac{\left.\mu^{2} \text { (Produkte }\right)-\mu^{2}(\text { Edukte })}{4 \pi \epsilon_{0} r^{3}} \frac{\partial \frac{n^{2}-1}{2 n^{2}+1}}{\partial \rho} .
$$


- $k_{\text {VER }}$ : Die Dichteabhängigkeit für den Schwingungsenergietransfer resultiert nur aus der Dichteabhängigkeit der Stoßzahl Z . Für die verdünnte Gasphase kann eine Stoßzahl $Z_{0}$ für harte Kugeln gemäß

$$
Z_{0}=\rho \sigma \sqrt{\frac{8 \pi k_{\mathrm{B}} T}{\mu}},
$$

angegeben werden. $\mu$ ist hierbei die reduzierte Masse, $\rho$ die Lösungsmitteldichte und $\sigma=\pi / 4\left[\sigma\left(\mathrm{CH}_{2} \mathrm{I}_{2}\right)+\sigma(\text { Lösungsmittel })\right]^{2}$ der Stoßquerschitt. Für Kohlendioxid wird der zur Berechnung des Stoßquerschnitts nötige Parameter $\sigma\left(\mathrm{CO}_{2}\right)$ aus dem 2-Zentren Lennard-Jones Potential (siehe Tabelle.5.1) abgeschätzt, in dem das $\mathrm{CO}_{2}$-Teilchen als Ellipsoid mit den drei Halbachsen der Länge $\sigma / 2, \sigma / 2$ und $(L+\sigma) / 2$ aufgefasst wird. Zur Berechnung von $\sigma\left(\mathrm{CO}_{2}\right)$ dient nun das arithmetische Mittel aus diesen drei Halbachsen (es ergibt sich ein Wert von $3.79 \AA$ ). Stoßzahlen in Lösung können im Rahmen des IBC (isolated binary collisions) Modells [144] ebenfalls durch Stoßzahlen für harte Kugeln ausgedrückt werden. Um den Einfluss der lokalen Dichte auf die Stosszahl einzubeziehen, wird $Z_{0}$ aus Gl.6.13 mit dem Maximum der radialen Verteilungsfunktion multipliziert:

$$
Z=Z_{0} \cdot g_{\max }(\rho)
$$

- $k_{\text {sek. }}$ : Der Geschwindigkeitskoeffizient $k_{\text {sek. }}$ in Kohlendioxid wird hier anhand Gleichung 6.5 durch ein einfaches Diffusionsmodell abgeschätzt. Für $\langle x\rangle$ wird der Wert von $3.79 \AA$ aus dem vorhergehendem Abschnitt eingesetzt. Der Diffusionskoeffizient des Iodatoms wird aus der Stokes-Einstein Beziehung gemäß

$$
D_{\mathrm{I}}=\frac{k_{\mathrm{B}} T}{6 \pi \eta a}
$$

berechnet. Für $\eta$ wird die Viskosität eingesetzt, die der lokalen Dichte entspricht: Bei einer homogenen Dichte von $10 \mathrm{~mol} / \mathrm{l}$ beträgt die lokale Dichte $g_{\max } \rho \simeq 19 \mathrm{~mol} / \mathrm{l}$ (siehe Abb.5.5). Die Viskosität von $\mathrm{CO}_{2}$ beträgt bei einer Dichte von $19 \mathrm{~mol} / \mathrm{l}$ und $35^{\circ} \mathrm{C} \sim 86 \mu \mathrm{Pa} \cdot \mathrm{s}$ [116]. Für $a$ wird der halbe Lennard-Jones Durchmesser des Iodatoms $\left(\sigma_{\mathrm{I}}=4.320 \AA[24]\right)$ eingesetzt. Mit diesen Werten ergibt sich $k_{\text {sek. }}$ zu $16.9 \cdot 10^{10} \mathrm{~s}^{-1}$. Die in dieser Art berechneten Geschwindigkeitskoeffizienten werden in Abb.6.15 mit den gemessenen Anstiegszeiten bei der Abfragewellenlänge von $405 \mathrm{~nm}$ verglichen. Nach Gleichung6.6 müssen die gemessenen $k$ bei allen Anregungswellenlängen über den berechneten $k_{\text {sek }}$ liegen, was jedoch bei den niedrigen Dichten nicht gegeben ist. Das hier vorgeschlagene einfache Diffusionsmodell überschätzt somit die Geschwindigkeit des sekundären Käfigausbruchs. Der sekundäre Käfigausbruch ist wahrscheinlich eher als ein Konkurrenzprozess aus diffusem Käfigausbruch und attraktiver Wechselwirkung des Käfigpaares aufzufassen [145, 146].

\subsubsection{Probewellenlängenabhängigkeit}

Als letzter Schritt wurde in dem Mechanismus in Abb6.14 die Schwingungsenergierelaxation des heißen Isomers eingefügt. Sichtbar ist die Schwingungsenergierelaxation an der spektra- 


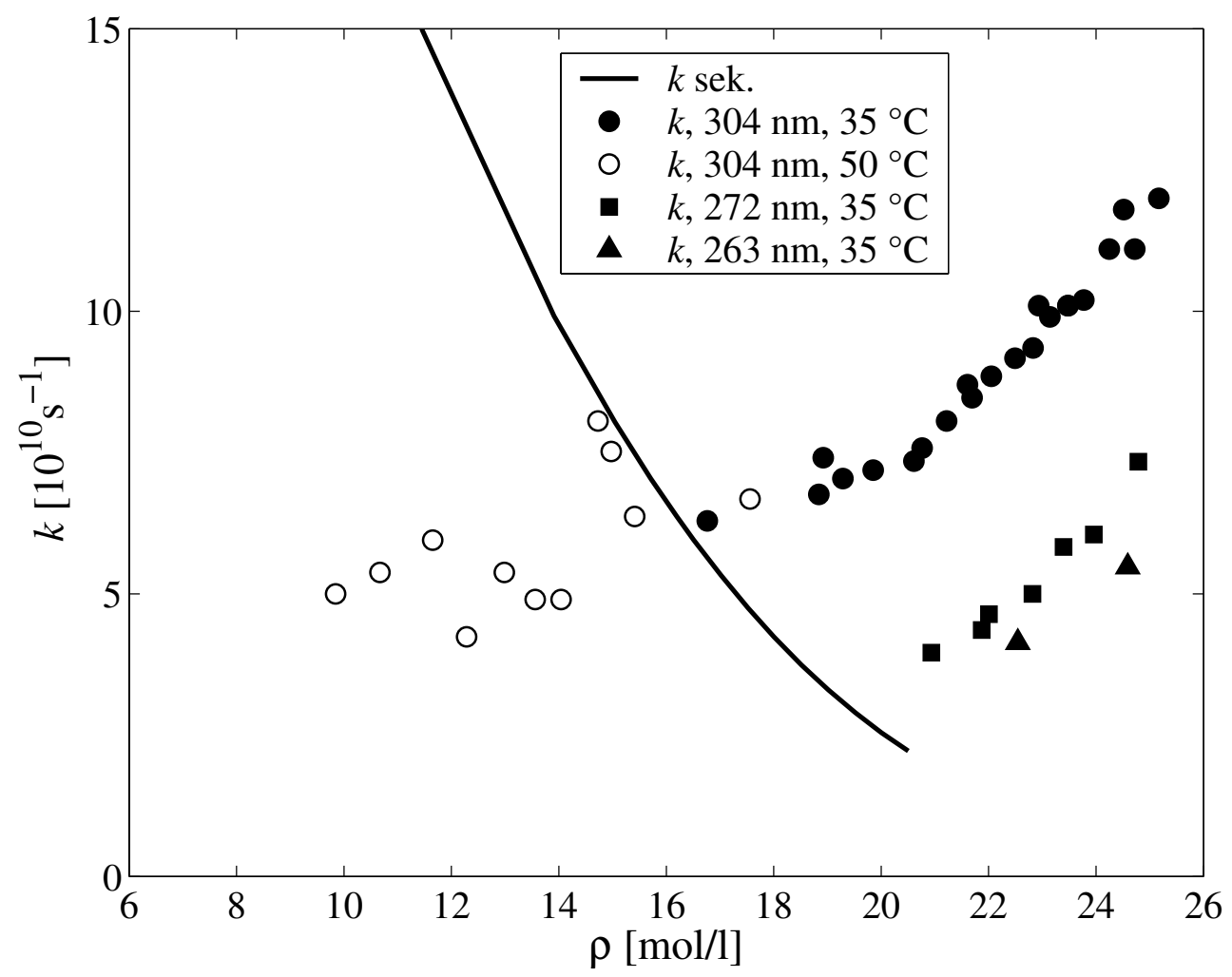

Abbildung 6.15: Vergleich der berechneten $k_{\text {sek. }}$ in $\mathrm{CO}_{2}$ mit den experimentellen Anstiegszeiten $k$ bei 263, 272 und $304 \mathrm{~nm}$ Anregung und $405 \mathrm{~nm}$ Probe.

len Evolution [114, 115] in den normierten Absorptionsspektren (siehe Abb.4.5, 4.22, A.2, A.4 und A.6): Die zeitaufgelösten und normierten Spektren zeigen an der roten Flanke einen zeitlichen Abfall und in der Mitte einen Anstieg. Dazwischen bei $405 \mathrm{~nm}$ befindet sich ein spektraler Bereich relativer Invarianz (quasi-isosbestischer Punkt). Anders ausgedrückt bedeutet dies, dass sich die Spektren mit fortschreitender Verzögerungszeit verschmälern.

Die Kühlung der Schwingungsenergie $\langle E\rangle(t)$ ist über die zeitliche Entwicklung des Extinktionskoeffizienten $\varepsilon$ messbar, jedoch muss dazu die Energieabhängigkeit von $\varepsilon$ bekannt sein. Temperaturabhängige Extinktionskoeffizienten werden überlicherweise aus Stoßwellenexperimenten erhalten. Unter der Annahme, dass bei gleicher mittlerer Energie thermisch und mikrokanonisch heiße Spektren identisch sind [115, 147], ist der Zusammenhang zwischen $\varepsilon$ und $\langle E\rangle$ gegeben. Weil das $\mathrm{CH}_{2} \mathrm{I}$ - I-Isomer jedoch eine metastabile Verbindung mit einer Lebensdauer im Nano- bis Mikrosekundenbereich ist, sind heiße Spektren auf diesem Weg nicht zugänglich.

Daher wird hier in folgender Weise aus den normierten Spektren die Größenordnung der Relaxationszeit abgeschätzt: Es wurde bei den Abfragewellenlängen $\geq 425 \mathrm{~nm}$ aus den rekonstruierten und normierten Spektren die Absorbanz in geringen Zeitabständen entnommen. Der zeitliche Abfall dieser normierten Absorbanz wurde mit einer einfach exponentiell abfallenden Funktion angepasst und die daraus erhaltene Lebensdauer $\tau_{\text {se }}$ in Abb.6.16 gegen die Wellenlänge aufgetragen.

Folgende Tendenzen sind aus der Auftragung ablesbar: 


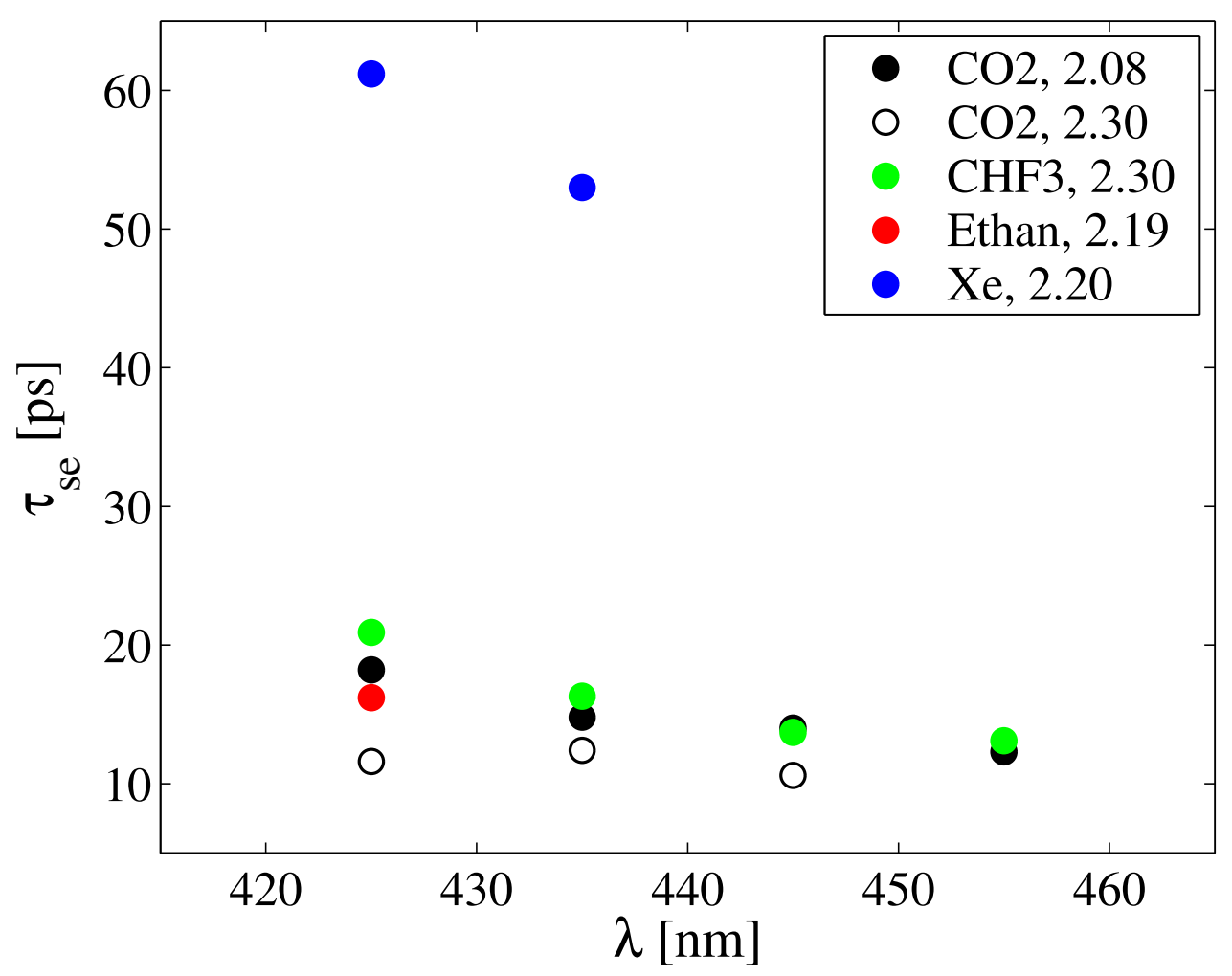

Abbildung 6.16: Auftragung der Lebensdauern der spektralen Evolution $\tau_{\text {se }}$ gegen die Abfragewellenlänge. Zusätzlich ist die reduzierte Dichte $\rho_{\mathrm{r}}$ angegeben.

- Die Lebensdauern der spektralen Evolution werden mit längeren Abfragewellenlängen kürzer.

- Mit 50-60 ps ist die Lebensdauer in Xenon wesentlich länger als in den polyatomaren Lösungsmitteln (10-25 ps).

- Die spektrale Evolution ist bei dem höheren $\mathrm{CO}_{2}$-Druck schneller.

Mit der Abkühlung des schwingungsheißen Isomers kann auch die Probewellenlängenabhängigkeit der Anstiegszeit (siehe Abb.4.13) erklärt werden: Längere Abfragewellenlängen proben energetisch höhere Schwingungszustände, welche durch den Energietransfer schneller entvölkert werden und somit zu einer Verkürzung der Anstiegszeit führen. Diese Beschleunigung mit längeren Abfragewellenlängen ist auch an der spektralen Evolution in Abb.6.16 sichtbar.

Die hier abgeschätzten Zeiten für den Energietransfer lassen sich nun mit den Relaxationszeiten nach C-H-Anregung von Diiodmethan in überkritischen $\mathrm{CO}_{2}$ und Xe vergleichen. Die VER Zeiten in $\mathrm{CO}_{2}$ fallen im untersuchten Dichtebereich $\left(0.7<\rho_{\mathrm{r}}<1.7\right)$ von 150 auf $50 \mathrm{ps}$ ab [136], während sie sich in Xe $\left(0.8<\rho_{\mathrm{r}}<1.6\right)$ von 600 auf $200 \mathrm{ps}$ verringern [148. Der VER im $\mathrm{CH}_{2} \mathrm{I}-\mathrm{I}$-Isomer ist dementsprechend gegenüber dem im Muttermolekül beschleunigt. Erklärbar ist dies dadurch, dass das Isomer mehr niederfrequente Moden als das Muttermolekül besitzt: Sowohl die C-I-I-Knick- als auch die I-I-Streckschwingung 
149] haben beide eine kleinere Frequenz als die niederfrequenteste Mode des $\mathrm{CH}_{2} \mathrm{I}_{2}$ (die I-C-I-Streckschwingung) [150].

\subsubsection{Anregungswellenlängenabhängigkeit}

Mit kürzeren Anregungswellenlängen nimmt nicht nur die innere Energie des $\mathrm{CH}_{2}$ I-Radikals zu, sondern auch die Ausbeute an angeregten Iodatomen. Im untersuchten Anregungsbereich von $340-263 \mathrm{~nm}$ steigt der Anteil an $\mathrm{I}^{*}$ von $\sim 0.05$ auf $\sim 0.4$. Weil jedoch nicht bekannt ist, in welcher Weise die angeregten Iodatome die Isomerisierung beeinflussen, wird für die folgende Betrachtung ihr Beitrag vernachlässigt.

Mit dem Vorgleichgewicht aus Abb.6.14 kann auch die Abhängigkeit der Bildungsgeschwindigkeit von der Anregungswellenlänge (siehe Abb.4.23) erklärt werden: Die Vorgleichgewichtskonstante $K$ ist eine Funktion der Schwingungstemperatur des $\mathrm{CH}_{2}$ I-Radikals. Mit zunehmender Schwingungstemperatur verschiebt sich das Gleichgewicht auf die Eduktseite, d. h. $K$ wird mit kürzeren Anregungswellenlängen kleiner. Nach Gl.6.6 wird dadurch auch $k$ kleiner, was sich mit der experimentellen Beobachtung deckt.

In Abb.6.17 wurden die Messreihen aus Abb.4.23 aufeinander skaliert. Bei der Messreihe mit der Anregungswellenlänge von $340 \mathrm{~nm}$ mit zweifach exponentiellem Anstieg wurde das amplitudengewichtete Mittel aus beiden Geschwindigkeitskoeffizienten aufgetragen. Die reziproken Skalierungsfaktoren wurden als quasi-Gleichgewichtskonstanten aufgefasst und in einer van't Hoff Auftragung in Abb.6.18 gegen die inverse Schwingungstemperatur des Radikals aufgetragen. Die innere Energie des Radikals wurde gemäß $\left\langle E_{\mathrm{CH}_{2} \mathrm{I}}\right\rangle=$ $0.8 \cdot\left(h c N_{\mathrm{A}} / \lambda-\Delta_{\text {dis }} H\right)$ abgeschätzt und mit diesem Wert die Schwingungstemperatur über G1.6.1 berechnet. Die Gleichgewichtskonstanten, inneren Energien und Schwingungstemperaturen sind in Tabelle 6.2 zusammengefasst. Aus der van't Hoff Auftragung wird schließlich eine Reaktionsenthalpie für die Bildung des CT-Komplex von $\Delta_{\mathrm{R}} H=-(43 \pm 7) \mathrm{kJ} / \mathrm{mol}$ abgeschätzt.

\begin{tabular}{c|c|c|l}
$\lambda / \mathrm{nm}$ & $\left\langle E_{\mathrm{CH}_{2} \mathrm{I}}\right\rangle / \mathrm{kJ} / \mathrm{mol}$ & $T_{\mathrm{vib}} / \mathrm{K}$ & \multicolumn{1}{|c}{$K$} \\
\hline 263 & 200 & 3200 & 0.455 \\
272 & 188 & 3000 & 0.550 \\
304 & 151 & 2400 & 1 \\
340 & 118 & 1900 & 1.37
\end{tabular}

Tabelle 6.2: Anregungswellenlängenabhängige Schwingungstemperaturen des $\mathrm{CH}_{2} \mathrm{I}$-Radikals und zugehörige quasi-Gleichgewichtskonstanten. 


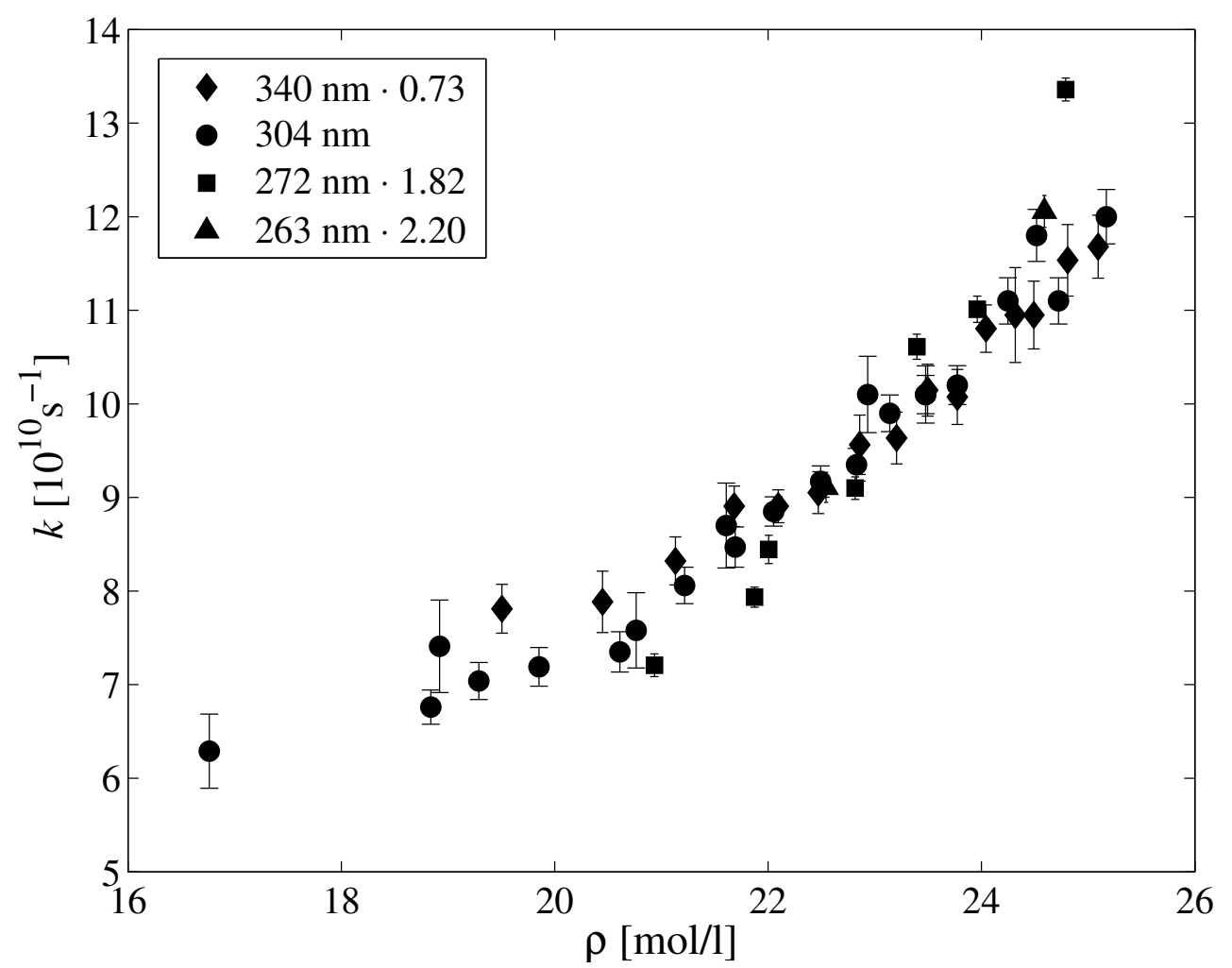

Abbildung 6.17: Auftragung der skalierten $k$ in $\mathrm{CO}_{2}$ bei verschiedenen Anregungswellenlängen $\left(405 \mathrm{~nm}\right.$ Probe, $\left.37^{\circ} \mathrm{C}\right)$.

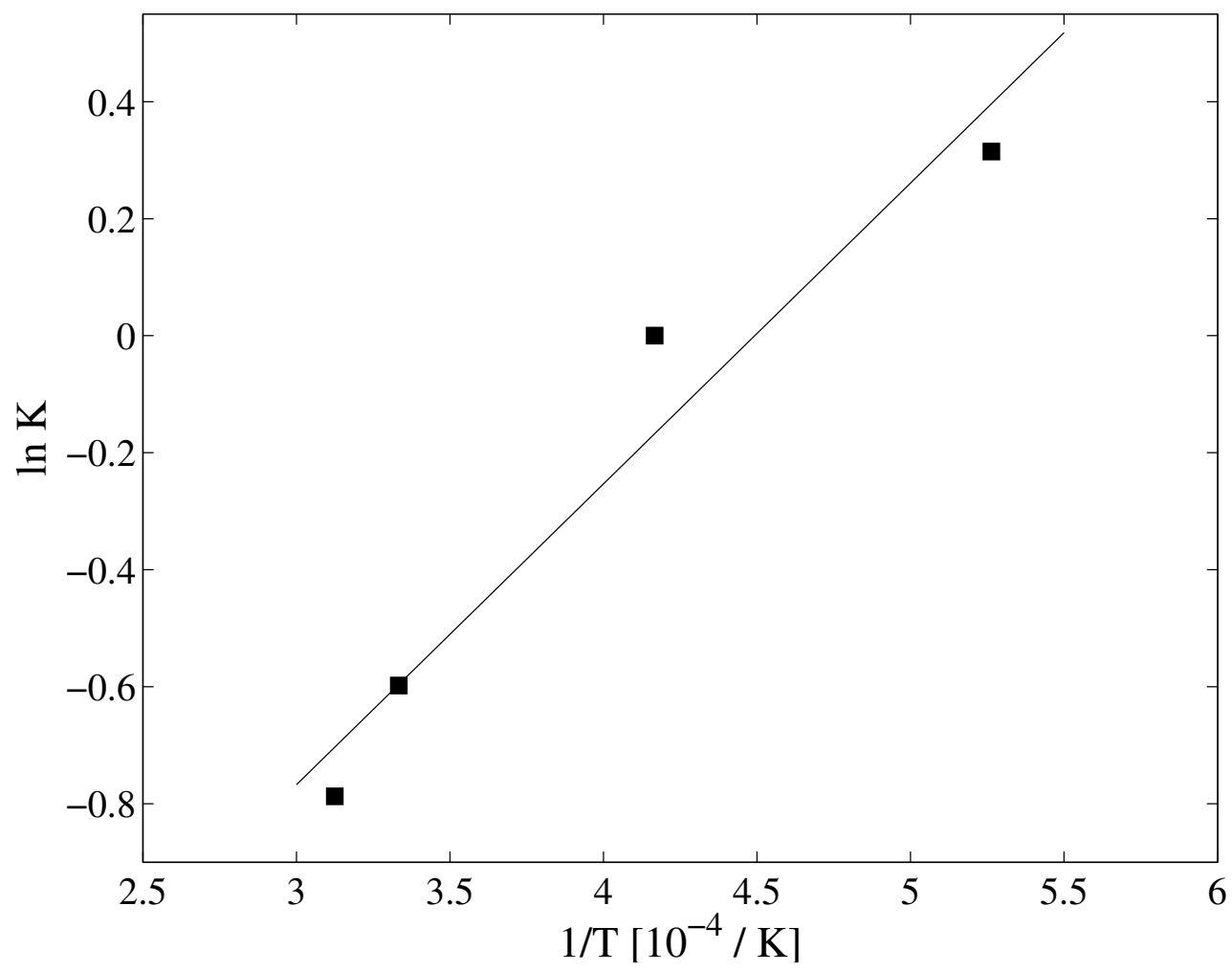

Abbildung 6.18: Van`t Hoff Auftragung zur Abschätzung der Komplexbildungsenthalpie. 


\subsection{Zusammenfassung}

In der vorliegenden Arbeit wurde ein Reaktionsmechanismus zur Interpretation der Dichteabängigkeit der photoinduzierten Isomerisierung von Diiodmethan aufgestellt. An dieser Stelle sollen die wichtigsten Gesichtspunkte noch einmal zusammengefasst werden.

Die gemessenen Quantenausbeuten konnten befriedigend durch das Quadrat der lokalen Dichte angepasst werden, was durch einen konsekutiven Käfigausbruch erklärt wurde. Der primäre Käfigeffekt verläuft dabei auf der Zeitskala der Translationskühlung und kann als rein kinematisch aufgefasst werden. Der sekundäre Käfigeffekt hingegen ergibt sich als Konkurrenzprozess zwischen Isomerisierung und diffusivem Ausbruch; die Zeitskala dieses Prozesses beträgt je nach experimentellen Bedingungen 5-50 ps. Zur Erklärung der Dichte- und Anregungswellenlängenabhängigkeit der Bildungsgeschwindigkeiten wurde ein Vorgleichgewicht zwischen unkomplexierten Photofragmenten und einem von uns postulierten Ladungstransferkomplex eingeführt. Dieses Gleichgewicht stellt sich direkt nach dem primären Käfigeffekt ein. Als experimentelle Hinweise für die Existenz des Ladungstransferkomplexes wurden das Minimum der Absorptionszeitprofile und die dichteabhängigen Anisotropieexperimente aufgeführt. Als letzter Schritt wurde im Reaktionsmechanismus die Schwingungskühlung des Isomers aufgenommen, welche sich in der spektralen Evolution widerspiegelt.

Nach diesem kinetischen Modell ergibt sich die Bildungsgeschwindigkeit aus den Geschwindigkeiten der einzelnen Teilschritte gemäß Gleichung 6.6 zu $k=k_{\text {sek. }}+K \cdot k_{\mathrm{VER}}$. Die Dichteabhängigkeit der Gleichgewichtskonstanten $K$ und des Geschwindigkeitskoeffizienten der Energierelaxation $k_{\text {VER }}$ wurde qualitativ beschrieben, während für $k_{\text {sek. }}$ eine Abschätzung durch ein einfaches Diffusionsmodell gegeben wurde. Die Abschätzung ergab einen zu großen Wert, was zu der Schlussfolgerung führte, dass eine attraktive Wechselwirkung der Photofragmente den diffusiven Käfigausbruch hemmt.

Für die Quantenausbeute des sekundären Käfigeinfangs wird nach Gleichung 6.7 eine wesentlich komplexere Dichteabhängigkeit als die ursprünglich angepasste lineare erwartet. Diese Inkonsistenz im Modell liegt aber jenseits dem, was experimentell aufgelöst werden kann.

Abschließend lässt sich feststellen, dass die experimentellen Ergebnisse zur Dichte- und Wellenlängenabhängigkeit der Photoisomerisierung von Diiodmethan qualitativ erklärt werden konnten. Im gesamten experimentell untersuchten Dichtebereich $\left(0.68<\rho_{\mathrm{r}}<2.5\right)$ wurde die Isomerisierung beobachtet. Die Frage, ob es eine kritische Dichte gibt, bei der der Isomerisierungskanal öffnet, konnte in dieser Arbeit nicht beantwortet werden. 


\section{Anhang}

\section{A.1 Kritische Daten der verwendeten Lösungsmittel}

\begin{tabular}{c|c|c|c} 
& $T_{\mathrm{c}} /{ }^{\circ} \mathrm{C}$ & $\rho_{\mathrm{c}} / \mathrm{mol} / \mathrm{l}$ & $p_{\mathrm{c}} /$ bar \\
\hline $\mathrm{CO}_{2}$ [116] & 30.98 & 10.63 & 73.8 \\
Ethan [116] & 32.18 & 6.87 & 48.72 \\
$\mathrm{CHF}_{3}$ [117] & 26.2 & 7.6 & 48.6 \\
Xenon [116] & 16.58 & 8.37 & 58.4
\end{tabular}

$T_{\mathrm{c}}$ : kritische Temperatur, $\rho_{\mathrm{c}}$ : kritische Dichte, $p_{\mathrm{c}}$ : kritischer Druck.

A.2 Zeitaufgelöste Absorptionsspektren in $\mathrm{Xe}, \mathrm{C}_{2} \mathrm{H}_{6}$ und $\mathrm{CHF}_{3}$ 


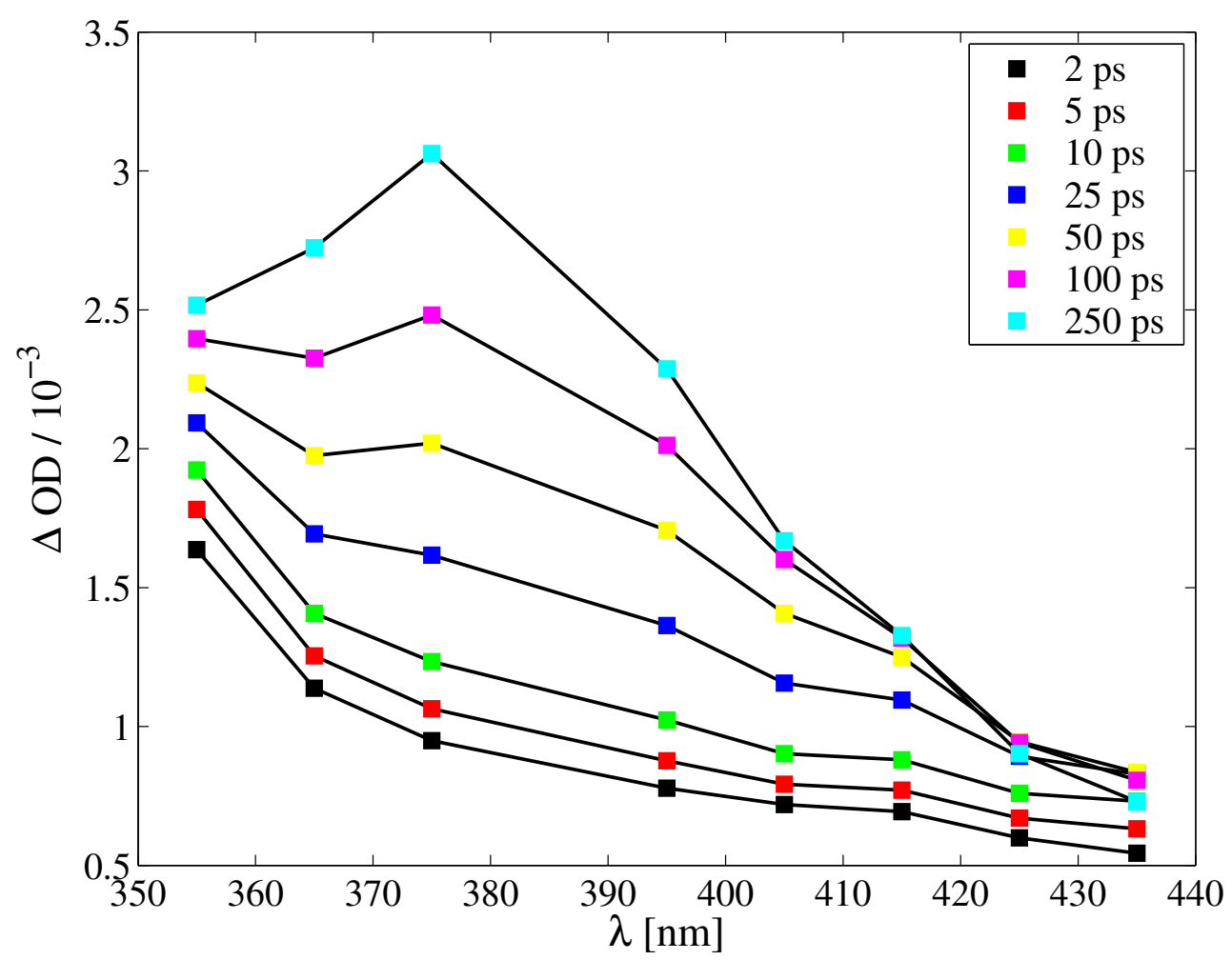

Abbildung A.1: Rekonstruiertes zeitaufgelöstes Spektrum von $\mathrm{CH}_{2} \mathrm{I}_{2}$ in $\mathrm{Xe}\left(505\right.$ bar, $\left.35^{\circ} \mathrm{C}\right)$.

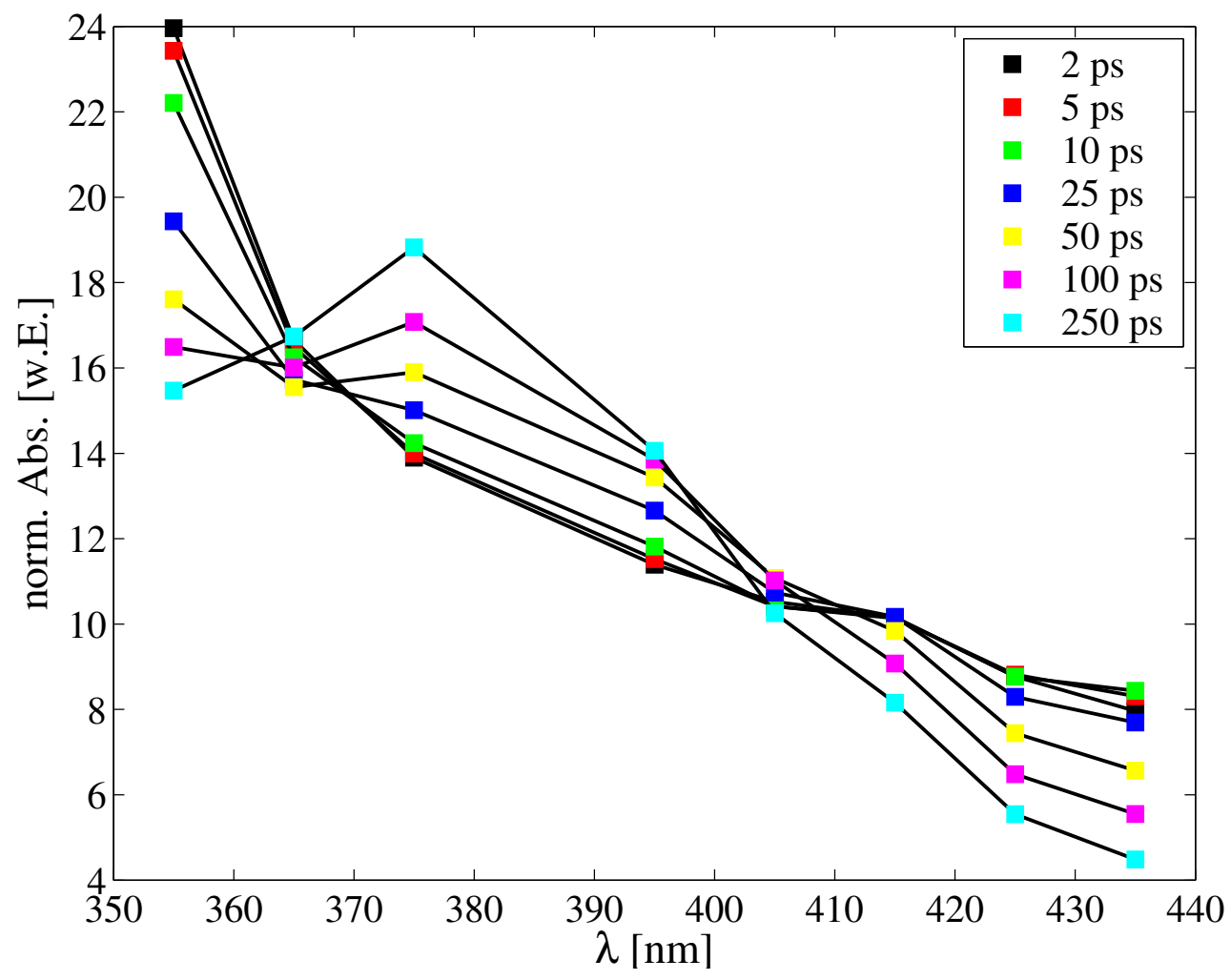

Abbildung A.2: Rekonstruiertes und normiertes zeitaufgelöstes Spektrum von $\mathrm{CH}_{2} \mathrm{I}_{2}$ in Xe (505 bar, $35^{\circ} \mathrm{C}$ ). 


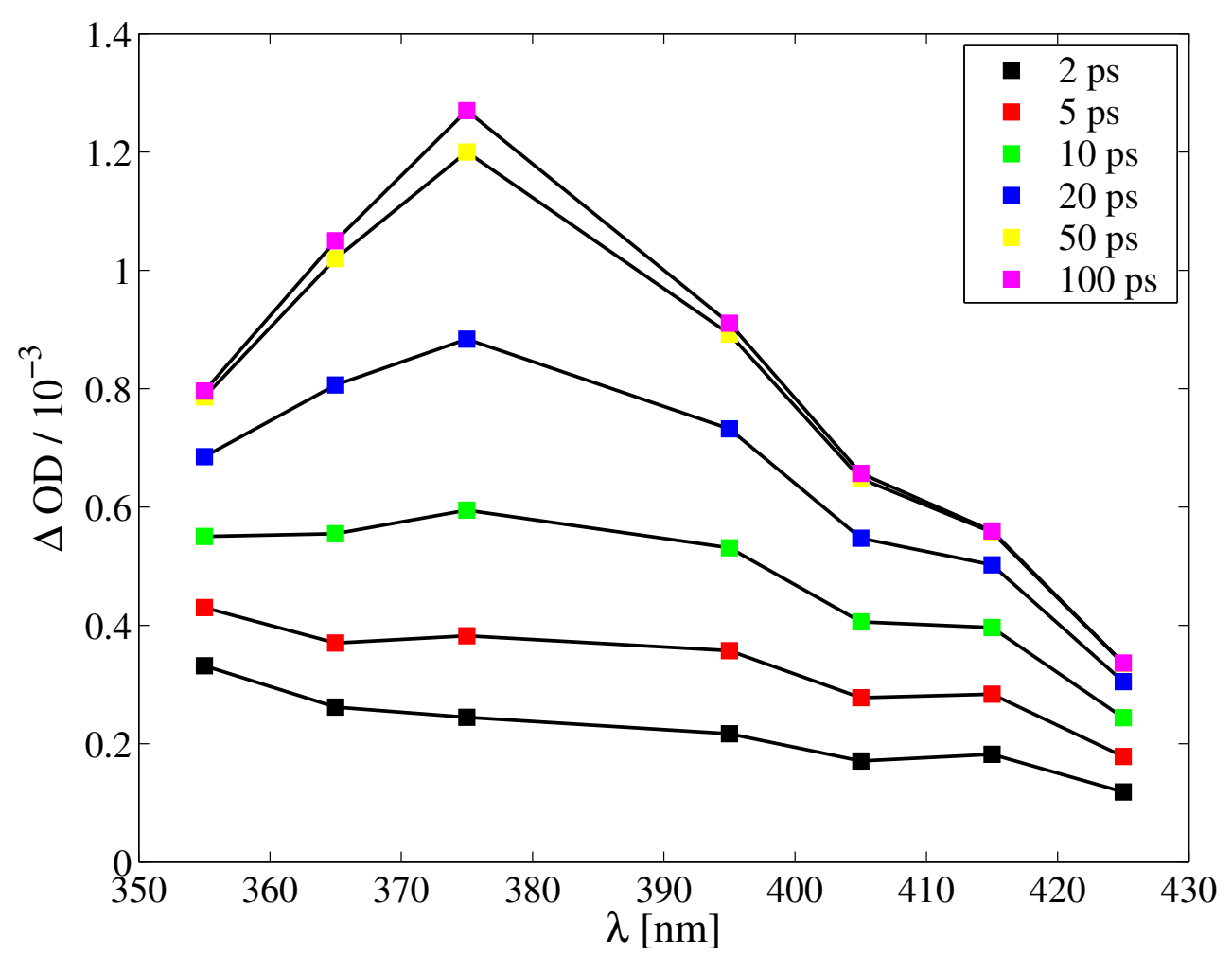

Abbildung A.3: Rekonstruiertes zeitaufgelöstes Spektrum von $\mathrm{CH}_{2} \mathrm{I}_{2}$ in Ethan (400 bar, $\left.37^{\circ} \mathrm{C}\right)$.

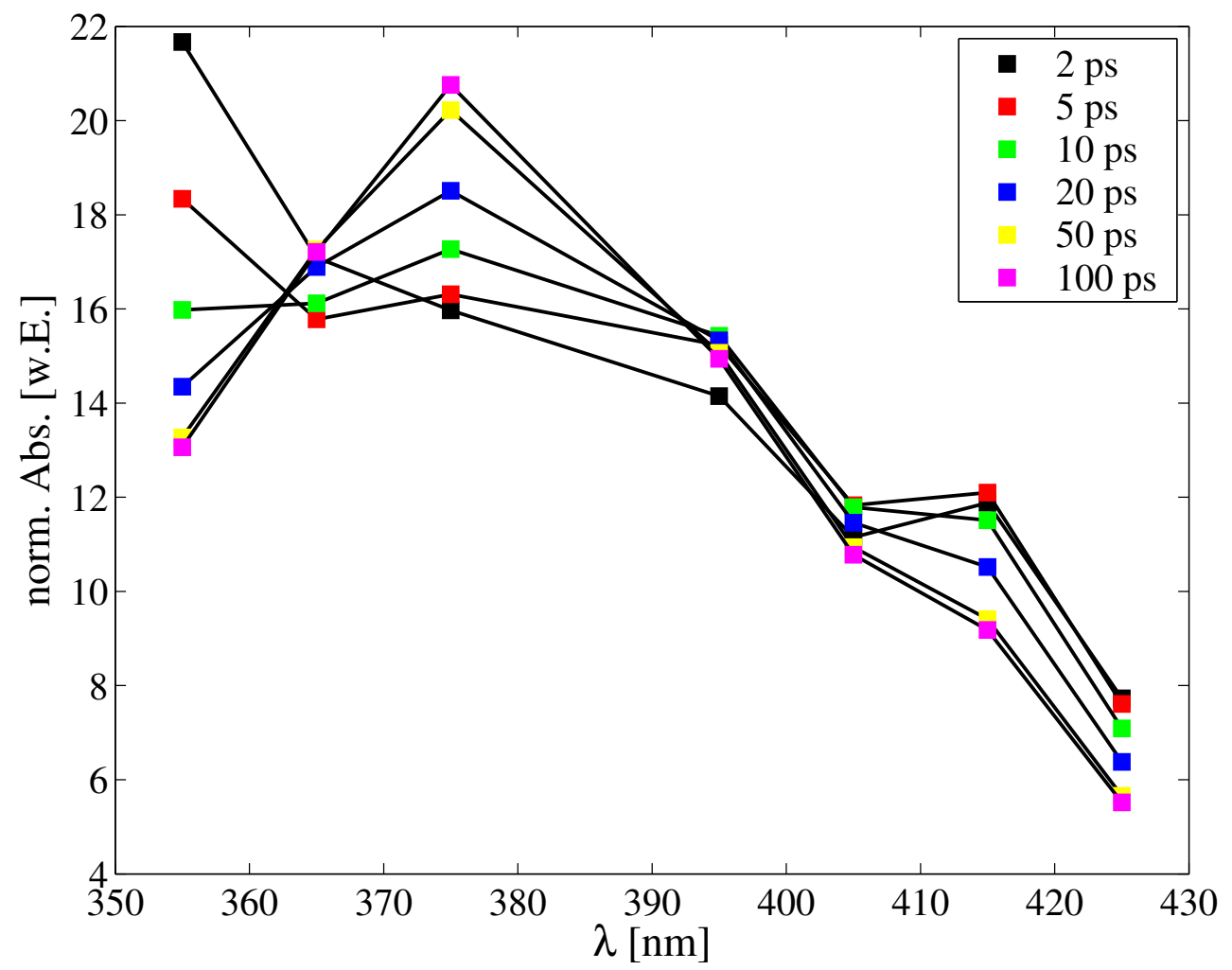

Abbildung A.4: Rekonstruiertes und normiertes zeitaufgelöstes Spektrum von $\mathrm{CH}_{2} \mathrm{I}_{2}$ in Ethan $\left(400\right.$ bar, $\left.37^{\circ} \mathrm{C}\right)$. 


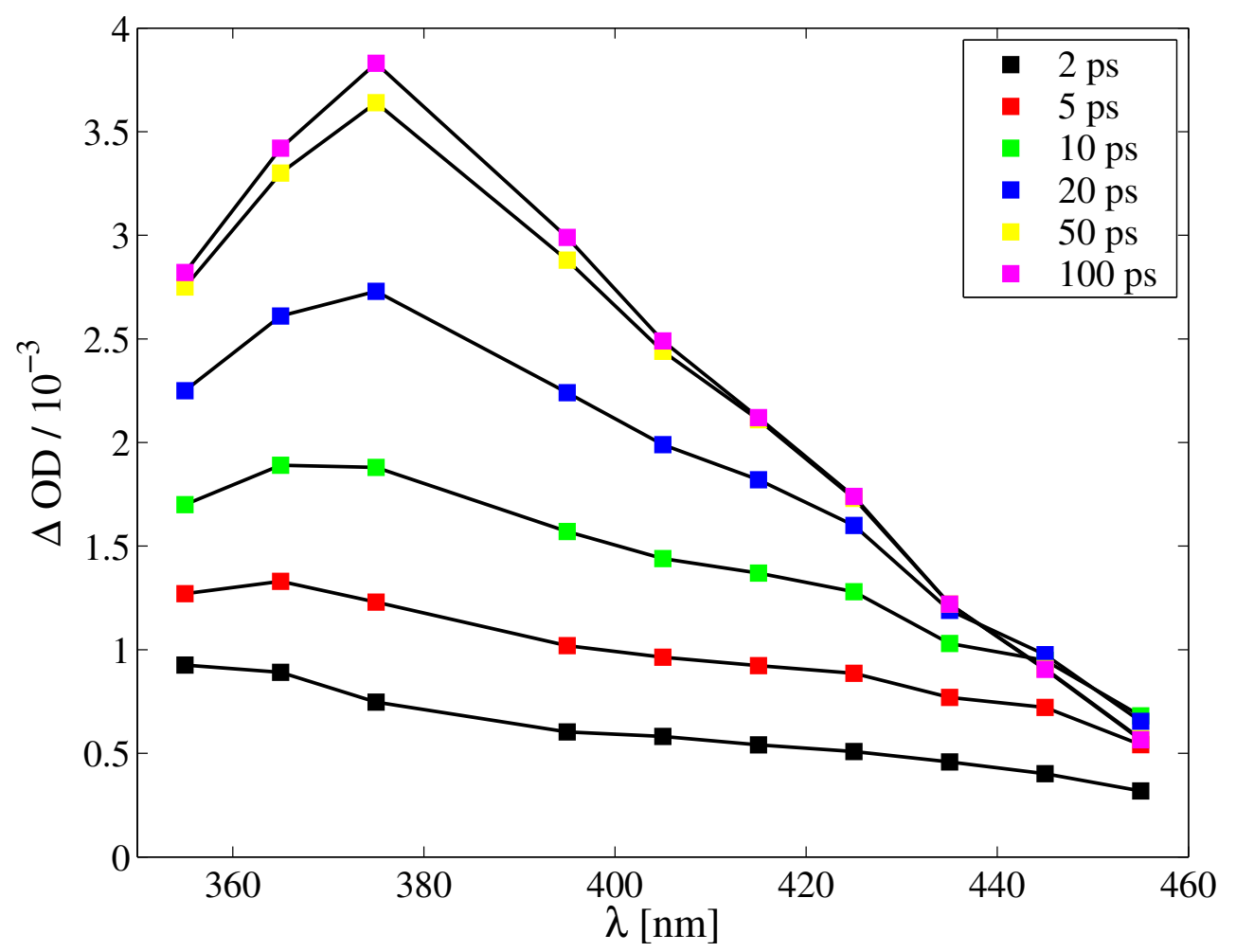

Abbildung A.5: Rekonstruiertes zeitaufgelöstes Spektrum von $\mathrm{CH}_{2} \mathrm{I}_{2}$ in $\mathrm{CHF}_{3}$ (510 bar, $37^{\circ} \mathrm{C}$ ).

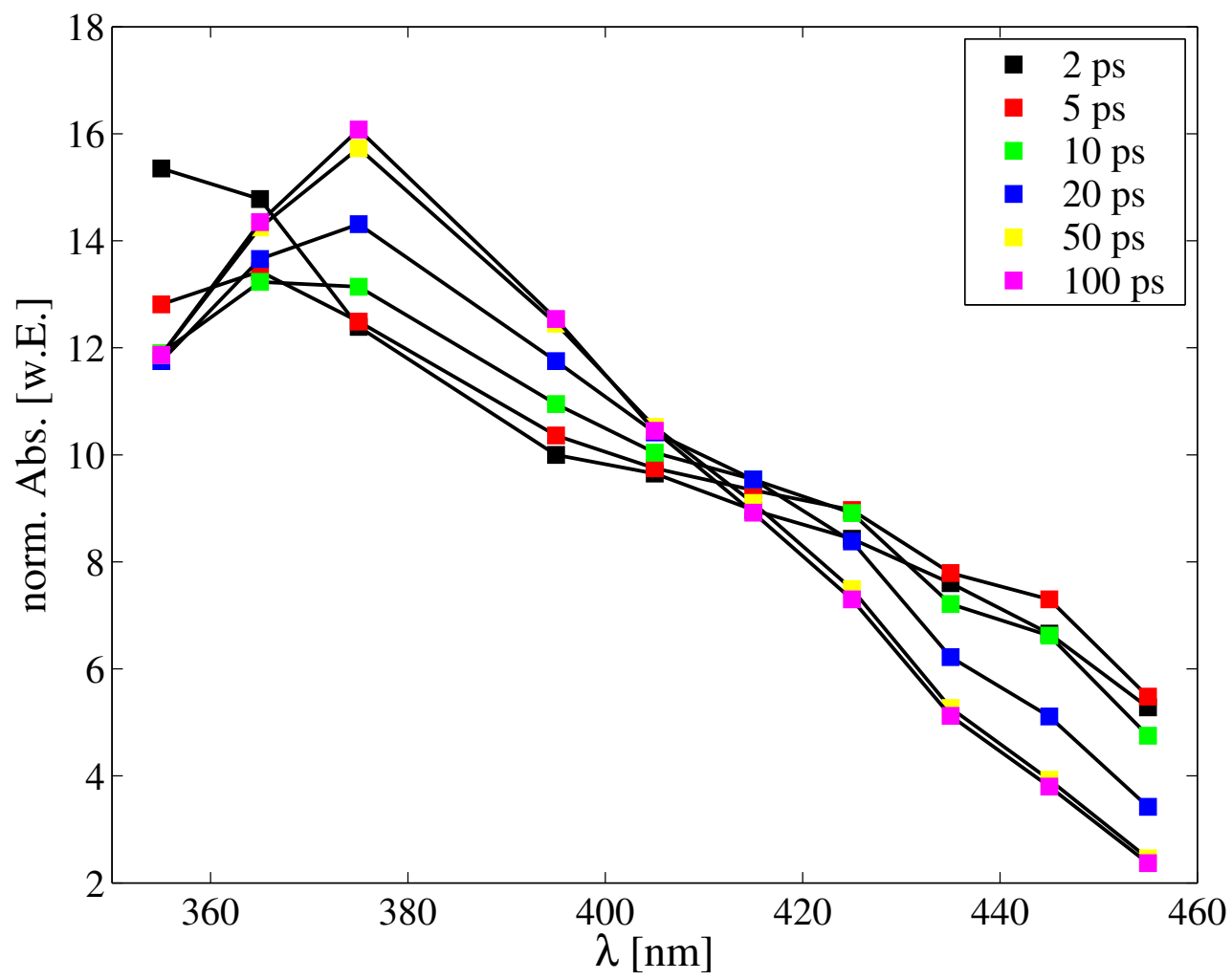

Abbildung A.6: Rekonstruiertes und normiertes zeitaufgelöstes Spektrum von $\mathrm{CH}_{2} \mathrm{I}_{2}$ in $\mathrm{CHF}_{3}\left(510\right.$ bar, $37^{\circ} \mathrm{C}$ ). 


\section{A.3 Sulzer-Wieland Modell zur Abschätzung der Radikalabsorption}

Für ein quasi-zweiatomiges Molekül ist der Absorptionsquerschnitt für einen Übergang von einem gebundenen zu einem repulsiven Zustand durch

$$
\sigma(\nu, T)=\sigma_{\max } \frac{\nu}{\nu_{0}} \sqrt{\tanh \left(\frac{\Theta_{0}}{2 T}\right)} \exp \left[-\tanh \left(\frac{\Theta_{0}}{2 T}\right)\left(\frac{\nu-\nu_{0}}{\Delta \nu_{0}}\right)^{2}\right]
$$

gegeben. $\sigma_{\max }$ ist das Maximum des Absorptionsquerschnitts, $\nu$ die Beobachtungsfrequenz, $\nu_{0}$ das Absorptionsmaximum bei $0 \mathrm{~K}, \Delta \nu_{0}$ und $\Theta_{0}=h c \omega_{0} / k_{\mathrm{B}}$ ist die Halbwertsbreite bzw. die charakteristische Temperatur der Schwingungsmode mit Frequenz $\omega_{0}$ bei $0 \mathrm{~K}$ und $T$ die Temperatur. Der Ausdruck kann auf polyatomare Systeme durch einen modifizierten Ausdruck für $\nu_{0}$ ausgeweitet werden: $\nu_{0, \text { eff }}=\nu_{0}-\omega_{0} /\left(\exp \left(\Theta_{0} / T\right)-1\right)$. Für das $\mathrm{CH}_{2}$ I-Radikal wurden die Parameter $\sigma_{\max }=1.9,4.0,2.9 \cdot 10^{-18} \mathrm{~cm}^{2}$ molecule ${ }^{-1}, \Delta \nu_{0}=1400,2000,2300 \mathrm{~cm}^{-1}$ und $\Theta_{0}=432$, 432, $576 \mathrm{~K}$ für den Peak der Wellenlänge 247, 283, bzw. $313 \mathrm{~nm}$ verwendet.

\section{A.4 Zeitaufgelöste Absorptionszeitprofile in $\mathrm{Xe}, \mathrm{C}_{2} \mathrm{H}_{6}$ und $\mathrm{CHF}_{3}$}
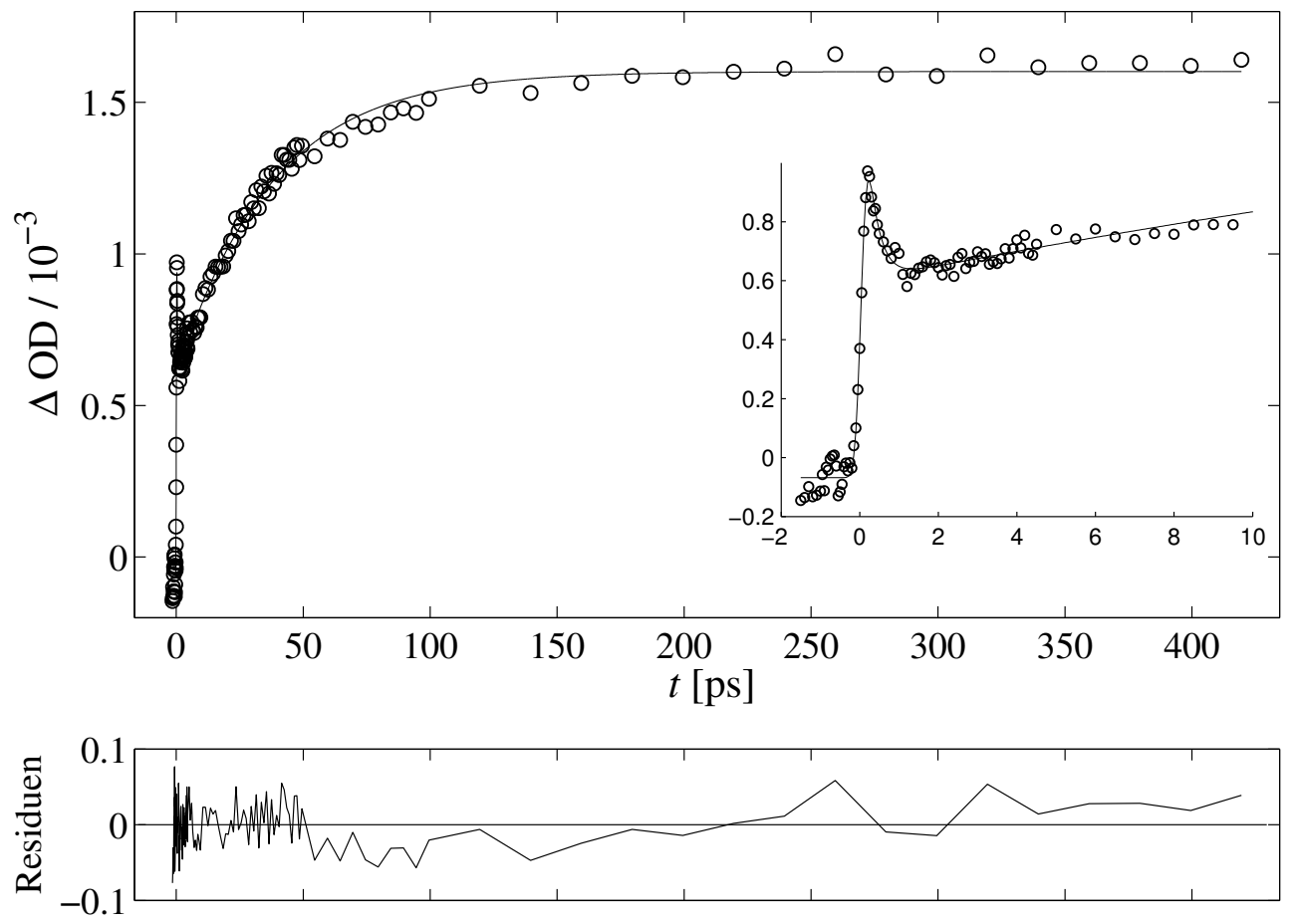

Abbildung A.7: Absorptionszeitprofil von $\mathrm{CH}_{2} \mathrm{I}_{2}$ in $\mathrm{Xe}\left(505\right.$ bar, $\left.35^{\circ} \mathrm{C}\right)$ bei $302 \mathrm{~nm}$ Anregung und $405 \mathrm{~nm}$ Abfrage. 

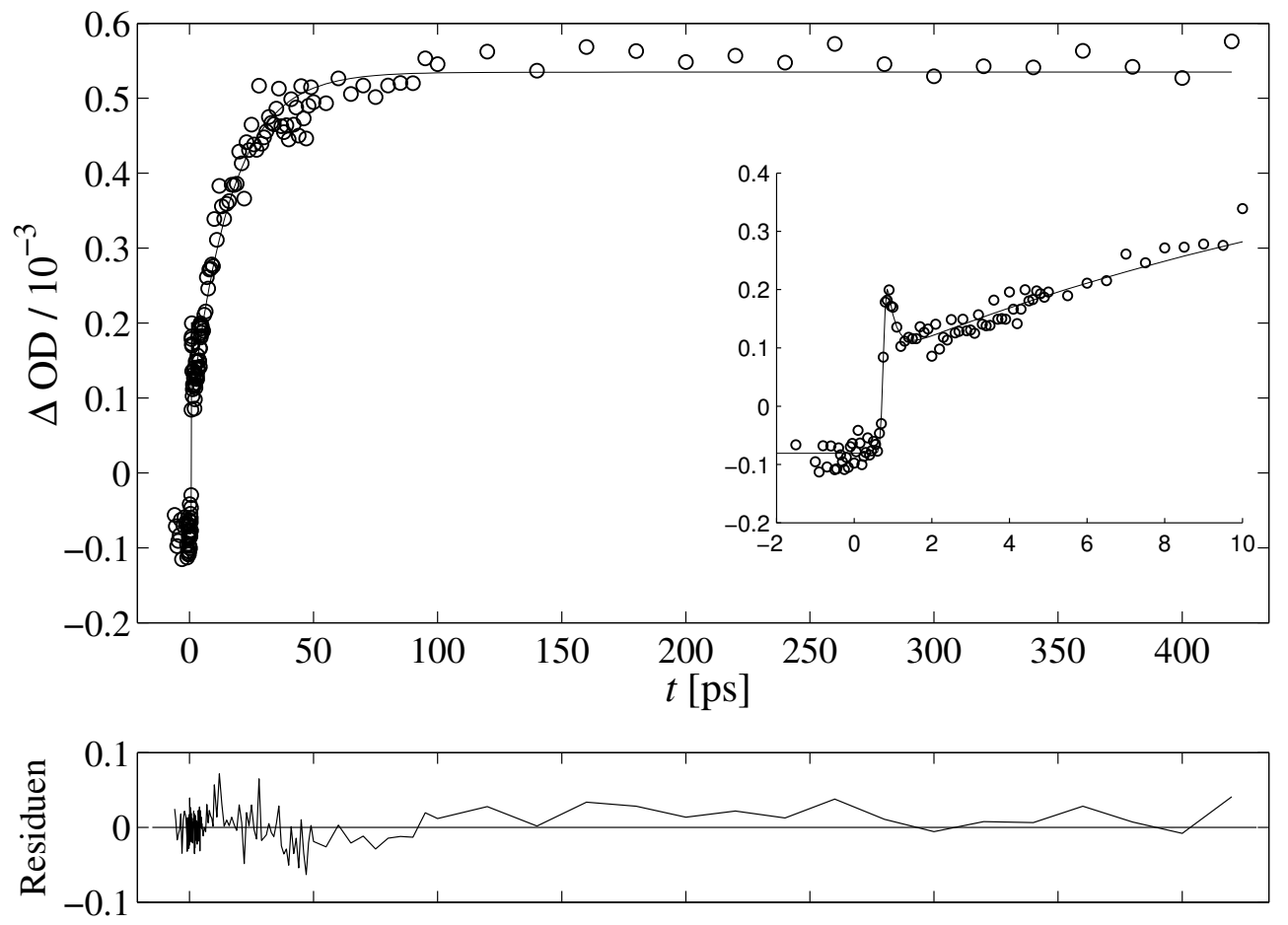

Abbildung A.8: Absorptionszeitprofil von $\mathrm{CH}_{2} \mathrm{I}_{2}$ in Ethan $\left(603\right.$ bar, $36^{\circ} \mathrm{C}$ ) bei $300 \mathrm{~nm}$ Anregung und $405 \mathrm{~nm}$ Abfrage.
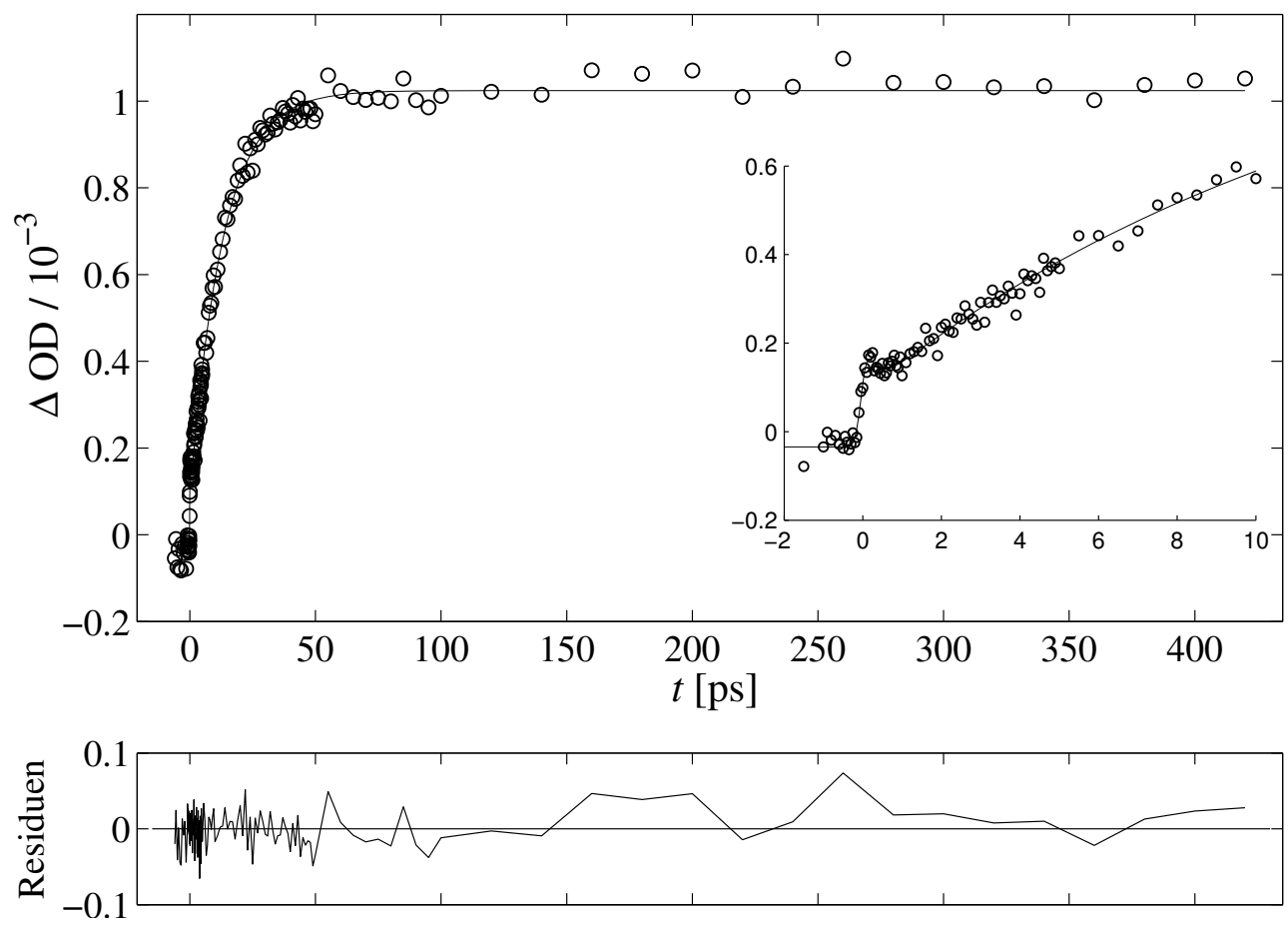

Abbildung A.9: Absorptionszeitprofil von $\mathrm{CH}_{2} \mathrm{I}_{2}$ in $\mathrm{CHF}_{3}\left(600\right.$ bar, $\left.30^{\circ} \mathrm{C}\right)$ bei $302 \mathrm{~nm}$ Anregung und $405 \mathrm{~nm}$ Abfrage. 


\section{A.5 Korrelation der Quantenausbeuten mit den lokalen Dichten in $\mathrm{Xe}, \mathrm{C}_{2} \mathrm{H}_{6}$ und $\mathrm{CHF}_{3}$}

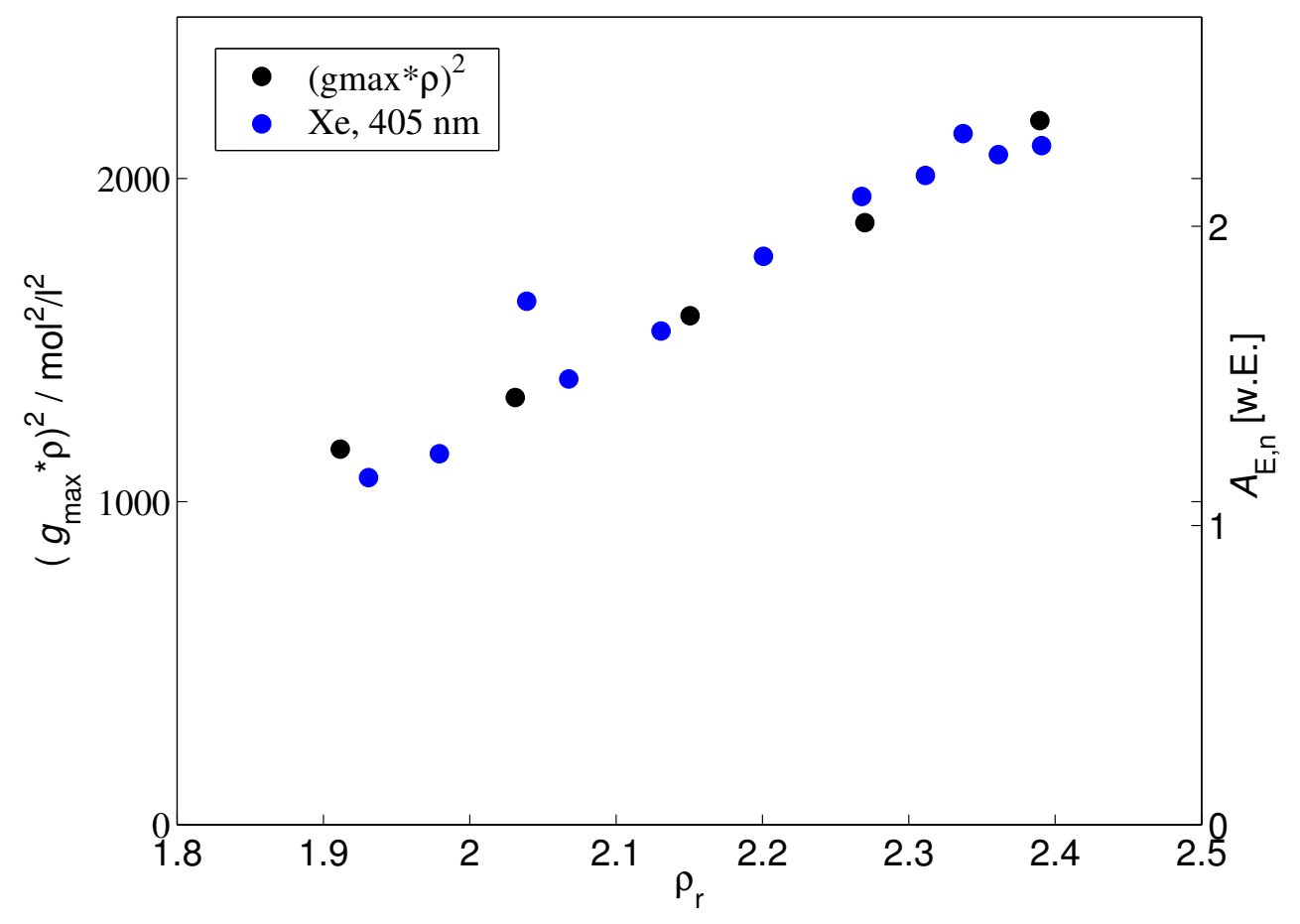

Abbildung A.10: Korrelation der $A_{\mathrm{E}, \mathrm{n}}$ in Xe bei $405 \mathrm{~nm}$ Abfrage mit $\left(g_{\max } \cdot \rho\right)^{2}$. 


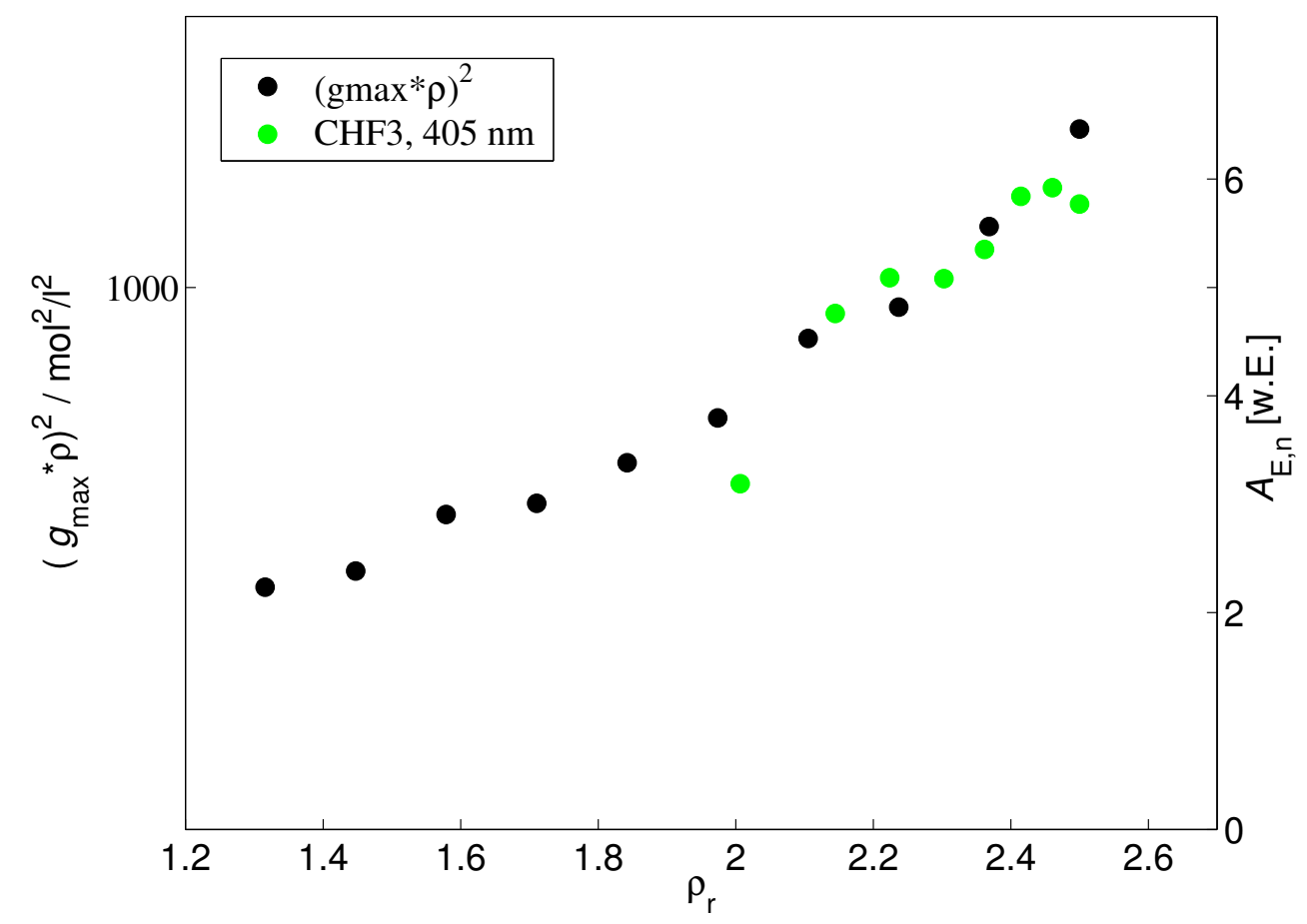

Abbildung A.11: Korrelation der $A_{\mathrm{E}, \mathrm{n}}$ in $\mathrm{CHF}_{3}$ bei $405 \mathrm{~nm}$ Abfrage mit $\left(g_{\max } \cdot \rho\right)^{2}$.

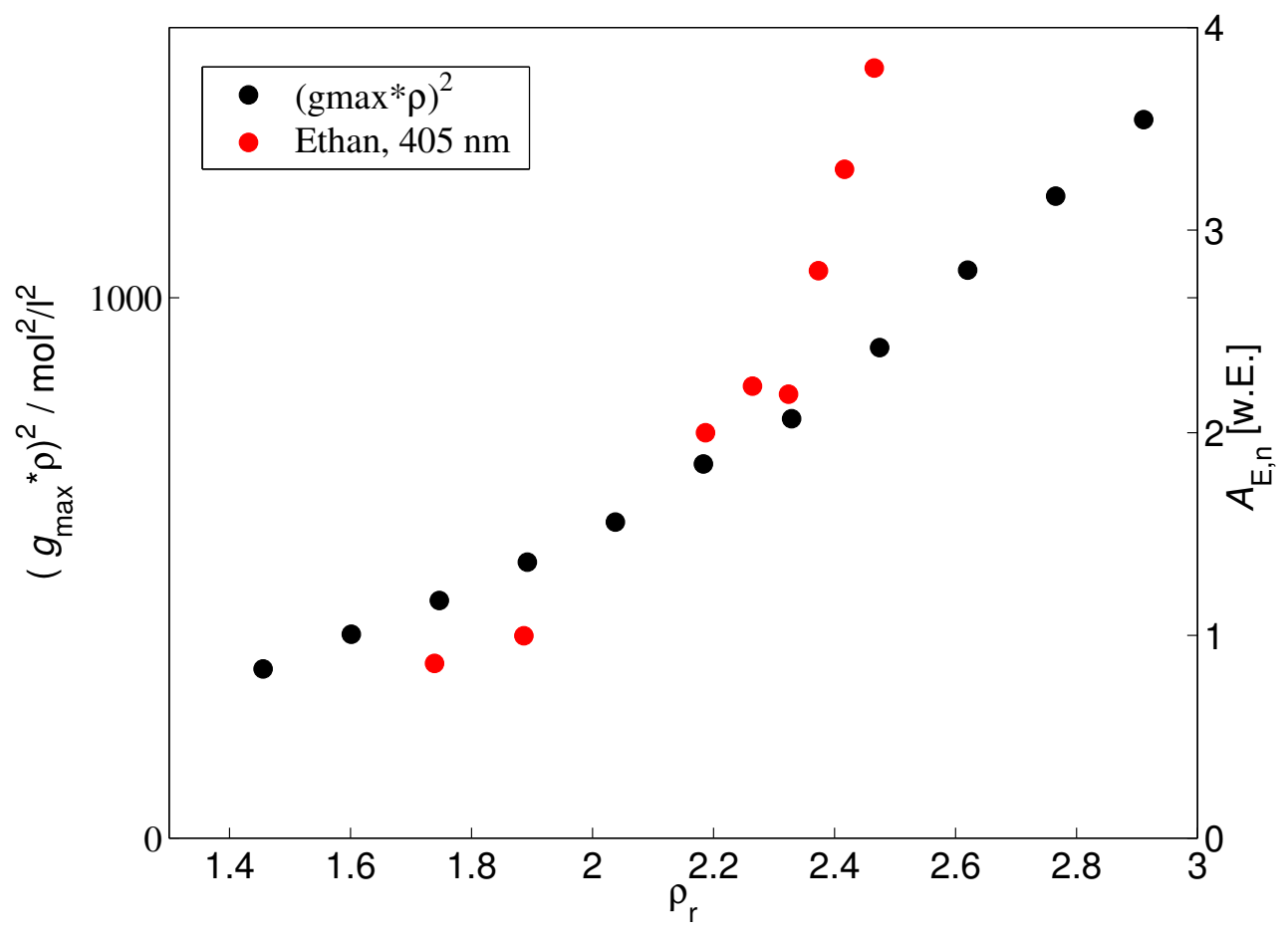

Abbildung A.12: Korrelation der $A_{\mathrm{E}, \mathrm{n}}$ in $\mathrm{C}_{2} \mathrm{H}_{6}$ bei $405 \mathrm{~nm}$ Abfrage mit $\left(g_{\max } \cdot \rho\right)^{2}$. 


\section{Literaturverzeichnis}

[1] E. Rabinowitsch, W. C. Wood, Trans. Faraday Soc., 1936, 32, 1381. 1

[2] R. M. Noyes, J. Am. Chem. Soc., 1955, 77, 2042. 3

[3] J. Franck, Trans. Faraday Soc., 1926, 21, 536. 3

[4] J. Franck, E. Rabinowitsch, Trans. Faraday Soc., 1934, 30, 120. 3

[5] J. Zimmerman, R. Noyes, J. Chem. Phys., 1950, 18, 658. 3

[6] R. M. Noyes, J. Chem. Phys., 1954, 22, 1349. 3

[7] F. W. Lampe, R. M. Noyes, J. Am. Chem. Soc., 1954, 76, 2140. 3

[8] D. Booth, R. M. Noyes, J. Am. Chem. Soc., 1960, 82, 1868. 3

[9] L. F. Meadows, R. M. Noyes, J. Am. Chem. Soc., 1960, 82, 1872. 3

[10] R. M. Noyes, Z. Elektrochm., 1960, 64, 153. 3

[11] K. Luther, J. Troe, Chem. Phys. Lett., 1974, 24, 85. 3

[12] K. Luther, J. Schroeder, J. Troe, U. Unterberg, J. Phys. Chem., 1980, 84, 3072. 4

[13] C. Lienau, A. H. Zewail, J. Phys. Chem., 1996, 100, 18629. 4, 5

[14] A. Materny, C. Lienau, A. H. Zewail, J. Phys. Chem., 1996, 100, 18650. 4

[15] M. Wall, A. N. Tarnovsky, T. Pascher, V. Sundström, E. Åkesson, J. Phys. Chem. A, 2003, 107, 211. 4

[16] X. Zheng, D. L. Phillips, Chem. Phys. Lett., 2000, 324, 175. 4

[17] J. Thøgersen, C. L. Thomsen, J. A. Poulsen, S. R. Keiding, J. Phys. Chem. A, 1998, 102, 4186. 4

[18] C. L. Thomsen, P. J. Reid, S. R. Keiding, J. Am. Chem. Soc., 2000, 122, 12795.4

[19] C. L. Thomsen, D. Madsen, J. Thøgersen, J. R. Byberg, S. R. Keiding, J. Chem. Phys., 1999, 111, 703. 4

[20] C. L. Thomsen, J. Thøgersen, S. R. Keiding, J. Chem. Phys., 2001, 114, 4099.4 
[21] C. L. Thomsen, D. Madsen, J. A. Poulsen, J. Thøgersen, S. J. Knak Jensen, J. Chem. Phys., 2001, 115, 9361. 4

[22] D. Madsen, C. L. Thomsen, J. A. Poulsen, S. J. Knak Jensen, J. Thøgersen, S. R. Keiding, E. B. Krissinel, J. Phys. Chem. A, 2003, 107, 3606. 4

[23] J. Larsen, D. Madsen, J.-A. Poulsen, T. D. Poulsen, S. R. Keiding, J. Thøgersen, J. Chem. Phys., 2002, 116, 7997. 4

[24] B. Otto, J. Schroeder, J. Troe, J. Chem. Phys., 1984, 81, 202. 4, 5, 79

[25] J. Troe, J. Phys. Chem., 1986, 90, 357. 4

[26] J. Schroeder, J. Troe, Ann. Rev. Phys. Chem., 1987, 38, 163. 4

[27] K. J. Naqvi, Razi K. ans Mork, S. Waldenström, J. Phys. Chem., 1980, 84, 1315. 5

[28] A. L. Harris, J. Brown, C. B. Harris, Ann. Rev. Phys. Chem., 1988, 39, 341. 6

[29] J. Tellinghuisen, J. Chem. Phys., 1973, 58, 2821. 6, 22

[30] K. Saenger, G. McClelland, D. Herschbach, J. Phys. Chem., 1981, 85, 3333. 6

[31] J. J. Valentini, J. B. Cross, J. Chem. Phys., 1982, 77, 572. 6

[32] A. E. S. Miller, C.-C. Chuang, H. C. Fu, K. J. Higgins, W. Klemperer, J. Chem. Phys., 1999, 111, 7844. 7

[33] J.-Y. Fang, C. C. Martens, J. Chem. Phys., 1996, 105, 9072. 7

[34] A. J. Conley, J.-Y. Fang, C. C. Martens, Chem. Phys. Lett., 1997, 272, 103. 7

[35] D. Schwarzer, J. Schroeder, C. Schröder, Z. Phys. Chem., 2001, 215, 183. 7, 67, 68, 71

[36] H. Ooe, Y. Kimura, M. Terazima, N. Hirota, J. Phys. Chem. A, 1999, 103, 7730. 8, 67

[37] D. L. Bunker, B. S. Jacobson, J. Am. Chem. Soc., 1972, 94, 1843. 8, 68

[38] J. N. Murrel, A. J. Stace, R. Dammel, J. Chem. Soc. Faraday Trans. 2, 1978, 74, 1532. 8, 68

[39] J. K. Brown, C. B. Harris, J. C. Tully, J. Chem. Phys., 1988, 89, 6687. 8, 68

[40] S. L. Baughcum, S. R. Leone, J. Chem. Phys., 1980, 72, 6531. 8, 9, 63

[41] M. Kawasaki, S. Lee, R. Bersohn, J. Chem. Phys., 1975, 63, 809. 8, 10

[42] H. Xu, Y. Guo, S. Liu, X. Ma, D. Dai, G. Sha, J. Chem. Phys., 2002, 117, 5722. 8, 9, 10, 27, 28 
[43] P. Kroger, P. Demou, S. Riley, J. Chem. Phys., 1976, 65, 1823. 8, 10,63

[44] J. Zhang, D. G. Imre, J. Chem. Phys., 1988, 89, 309. 8, 10

[45] S. R. Cain, R. Hoffmann, E. R. Grant, J. Phys. Chem., 1981, 85, 4046. 8

[46] U. Marvet, M. Dantus, Chem. Phys. Lett., 1996, 256, 57. 8

[47] U. Marvet, Q. Zhang, E. J. Brown, M. Dantus, J. Chem. Phys., 1998, 109, 4415 . 8

[48] U. Marvet, Q. Zhang, M. Dantus, J. Phys. Chem. A, 1998, 102, 4111. 8

[49] J. B. Koffend, S. R. Leone, Chem. Phys. Lett., 1981, 81, 136. 8, 9, 11

[50] T. F. Hunter, K. S. Kristjansson, Chem. Phys. Lett., 1982, 90, 35. 9, 11

[51] H. Hofmann, S. R. Leone, J. Chem. Phys., 1978, 69, 641. 9

[52] J. Sehested, T. Ellermann, O. J. Nielsen, Int. J. Chem. Kinet., 1994, 26, 259. 9, 12, 22

[53] E. Villenave, R. Lesclaux, Chem. Phys. Lett., 1995, 236, 376. 9, 12, 14,63

[54] D. R. Lide, CRC Handbook of Chemistry and Physics, CRC Press, 2000-2001. 9, 62

[55] W. B. DeMore, D. M. Golden, R. F. Hampson, C. J. Howard, C. E. Kolb, M. J. Kurylo, M. J. Molina, A. R. Ravishankara, S. P. Sander, Chemical Kinetics and Photochmical Data for Use in Stratospheric Modeling, Evaluation Number 12, JPL Publication 97-4, NASA National Aeronautics and Space Administration / Jet Propulsion Laboratory, California Institute of Technology, Pasadena, CA, 1997. 9, 62

[56] W. M. Kwok, D. L. Phillips, J. Chem. Phys., 1996, 104, 2529. 11

[57] G. Maier, H. P. Reisenauer, Angew. Chem., 1986, 98, 829. 11, 13,14

[58] G. Maier, H. P. Reisenauer, J. Hu, L. J. Schaad, B. A. J. Hess, J. Am. Chem. Soc., 1990, 112, 5117. 11, 13,14

[59] M. N. Glukhovtsev, R. D. Bach, Chem. Phys. Lett., 1997, 269, 145. 11, 12,62

[60] A. E. Orel, O. Kühn, Chem. Phys. Lett., 1999, 304, 285. 11

[61] A. N. Tarnovsky, J.-L. Alvarez, A. P. Yartsev, V. Sundström, E. Åkesson, Chem. Phys. Lett., 1999, 312, 121. 12, 28, 30, 61, 64

[62] M. Odelius, M. Kadi, J. Davidsson, A. N. Tarnovsky, J. Chem. Phys., 2004, 121, 2208. 12

[63] A. N. Tarnovsky, V. Sundström, E. Åkesson, T. Pascher, J. Phys. Chem. A, 2004, 108, 237. 12, 13, 30, 41, 61.

[64] X. Zheng, D. L. Phillips, J. Phys. Chem. A, 2000, 104, 6880. 13, 63, 66 
[65] W. M. Kwok, C. Ma, A. W. Parker, D. Phillips, M. Towrie, P. Matousek, D. L. Phillips, J. Chem. Phys., 2000, 113, 7471. 13

[66] S. J. Lee, R. Bersohn, J. Phys. Chem., 1982, 86, 728. 14

[67] S.-Q. Man, W. M. Kwok, D. L. Phillips, J. Chem. Phys., 1996, 105, 5842. 14, 15

[68] L. Butler, E. Hintsa, Y. T. Lee, J. Chem. Phys., 1986, 84, 4104. 14

[69] L. Butler, E. Hintsa, S. Shane, Y. T. Lee, J. Chem. Phys., 1987, 86, 2051. 14

[70] X. Zheng, D. L. Phillips, J. Chem. Phys., 2000, 113, 3194. 14

[71] W. M. Kwok, C. Ma, D. Phillips, A. W. Parker, M. Towrie, P. Matousek, D. L. Phillips, Chem. Phys. Lett., 2001, 341, 292. 14

[72] S.-Q. Man, W. M. Kwok, D. L. Phillips, J. Phys. Chem., 1995, 99, 15705.15

[73] A. N. Tarnovsky, M. Wall, M. Gustafsson, N. Lascoux, V. Sundström, E. Åkesson, J. Phys. Chem. A, 2002, 106, 5999. 15

[74] Y. Arai, T. Sako, Y. Takebayashi, Supercritical Fluids, Springer, Berlin Heidelberg, 2002. 15

[75] C. A. Eckert, B. L. Knutson, P. G. Debenedetti, Nature, 1996, 383, 313. 16

[76] K. Nishikawa, A. A. Arai, T. Morita, J. Supercritical Fluids, 2004, 30, 249. 16

[77] S. C. Tucker, Chem. Rev., 1999, 99, 391. 16, 17

[78] P. G. Debenedetti, R. S. Mohamed, J. Chem. Phys., 1989, 90, 4528. 16

[79] I. B. Petsche, P. G. Debenedetti, J. Chem. Phys., 1989, 91, 7075. 16

[80] I. B. Petsche, P. G. Debenedetti, J. Phys. Chem., 1991, 95, 386. 16, 17

[81] O. Kajimoto, Chem. Rev., 1999, 99, 355. 17, 53

[82] W. Song, R. Biswas, M. Maroncelli, J. Phys. Chem. A, 2000, 104, 6924. 17

[83] W. Song, M. Maroncelli, Chem. Phys. Lett., 2003, 378, 410. 17

[84] R. S. Berry, S. A. Rice, J. Ross, Physical Chemistry, 2 edn., Oxford University Press, 2000. 17

[85] N. Metropolis, A. W. Rosenbluth, M. N. Rosenbluth, A. H. Teller, E. Teller, J. Chem. Phys., 1953, 21, 1087. 17

[86] M. P. Allen, D. J. Tildesley, Computer Simulation of Liquids, Clarendon Press, Oxford, 1987. 17, 51, 52

[87] D. Schwarzer, J. Troe, M. Zerezke, J. Chem. Phys., 1997, 107, 8380. 17, 76 
[88] C. Grimm, M. Kling, J. Schroeder, J. Troe, J. Zerbs, Isr. J. Chem., 2003, 43, 305. 19,65

[89] B. Abel, J. Aßmann, P. Botschwina, M. Buback, M. Kling, R. Oswald, S. Schmatz, J. Schroeder, T. Witte, J. Phys. Chem. A, 2003, 107, 5157. 19

[90] B. Abel, J. Aßmann, M. Buback, M. Kling, S. Schmatz, J. Schroeder, Angew. Chem. Int. Ed., 2003, 42, 399. 19

[91] M. Kling, Experimentelle und Theoretische Untersuchungen der FemtosekundenKinetik des Photoinduzierten Zerfalls Aromatischer Peroxidverbindungen, Dissertation, Georg-August-Universität Göttingen, 2002. 19

[92] C. Grimm, Femtosekunden Photolyse von Diiodmethan in überkritischen Fluiden: Konkurrenz zwischen Photodissoziation und Photoisomerisierung, Dissertation, Georg-August-Universität Göttingen, 2003, http://webdoc.sub.gwdg.de/diss/ 2004/grimm/grimm.pdf. 19, 22, 42

[93] J. Zerbs, Untersuchungen zur Ultrakurzzeitdynamik von intramolekularen Ladungsübertragungsprozessen von Aminobenzonitrilderivaten in Lösung, Diplomarbeit, Georg-August-Universität Göttingen, 2002. 19

[94] P. Wagener, Käfigdynamik der Photodissoziation von Diiodmethan: Druck- und Temperaturabhängigkeit in überkritischen Lösungsmitteln, Diplomarbeit, Georg-AugustUniversität Göttingen, 2004. 19, 27

[95] Clark-MXR Inc., CPA-2001: User's Manual Version 1.0, 1998. 19, 21

[96] P. Maine, D. Strickland, P. Bado, M. Pesset, G. Mouru, IEEE J. Quantum Electron., 1988, 24, 398. 20

[97] K. Tamura, M. Nakazawa, Appl. Phys. Lett., 1995, 67, 3691. 20

[98] K. Tamura, E. P. Ippen, H. A. Haus, Appl. Phys. Lett., 1995, 67, 158. 20

[99] W. Lauterborn, T. Kurz, Coherent Optics, 2 edn., Springer, 2003. 20, 21

[100] C. Rulliere, Femtosecond Laser Pulses, 2 edn., Springer, 2003. 20

[101] F. Krausz, M. E. Fermann, T. Brabec, P. F. Curley, M. Hofer, M. H. Ober, C. Spielmann, E. Wintner, A. Schmidt, IEEE J. Quantum Electron., 1992, 28, 2097. 20

[102] U. Keller, W. H. Knox, G. W. 'tHooft, IEEE J. Quantum Electron., 1992, 28, 2123. 20

[103] C. Spielmann, M. Lenzner, A. Stingl, R. Szipöcs, F. Krausz, Phys. Bl., 1995, 51, 289. 20

[104] J. Piel, E. Riedle, Optics Letters, 1997, 22, 1494. 21 
[105] J. Piel, M. Beutter, E. Riedle, Optics Letters, 2000, 25, 180. 21

[106] Jobin Yvon GmbH, Manual: NOPA Nichtkollinear gepumptes OPA-System, 2001. 21

[107] Light Conversion, TOPAS: User's Manual, 2001. 21

[108] Light Conversion, TOPAS 4/800 Alignment Guide, 2003. 21

[109] B. Valeur, Molecular Fluorescence. Principles and Applications, Wiley-VCH, 2001. 23

[110] G. R. Fleming, Chemical Applications of Ultrafast Spectroscopy, Oxford University Press, 1985. 23, 65

[111] B. J. Schwartz, J. C. King, J. Z. Zhang, C. B. Harris, Chem. Phys. Lett., 1993, 203, 503. 28

[112] K.-i. Saitow, Y. Naitoh, K. Tominaga, K. Yoshihara, Chem. Phys. Lett., 1996, 262, 621. 28

[113] P. Sulzer, K. Wieland, Helv. Phys. Acta, 1951, 25, 653. 30

[114] L. Brouwer, J. Troe, Chem. Phys. Lett., 1981, 82, 1. 30, 80

[115] L. Brouwer, H. Hippler, L. Lindemann, J. Troe, J. Phys. Chem., 1985, 89, 4608. 30. 80

[116] National Institute of Standards and Technology, NIST Chemistry WebBook, NIST Standard Reference Database, 69 edn., 2003, http://webbook.nist.gov/chmistry/. 34, 41, 65, 79, 85

[117] K. Reuter, S. Rosenzweig, E. U. Franck, Physica A, 1989, 156, 294. 39, 85

[118] W. Song, N. Patel, M. Maroncelli, J. Phys. Chem. B, 2002, 106, 8783. 39

[119] R. G. Rubio, J. A. Zollweg, W. B. Streett, Ber. Bunsenges. Phys. Chem., 1989, 93, 791. 42

[120] J. Stoll, J. Vrabec, H. Hasse, Fluid Phase Equilib., 2001, 179, 339. 51

[121] J. Vrabec, J. Stoll, H. Hasse, J. Phys. Chem. B, 2001, 105, 12126. 51,52

[122] J. Stoll, J. Vrabec, H. Hasse, Fluid Phase Equilib., 2003, 209, 29. 51

[123] J. Stoll, J. Vrabec, H. Hasse, J. Chem. Phys., 2003, 119, 11396. 51, 52

[124] C. G. Gray, K. E. Gubbins, Theory of Molecular Fluids, Clarendon, Oxford, 1984. 51,52

[125] K. Refson, Comp. Phys. Commun., 2000, 126, 310. 53

[126] L. Nikowa, D. Schwarzer, J. Troe, J. Schroeder, J. Chem. Phys., 1992, 97, 4827.61 
[127] T. Lenzer, K. Oum, J. Schroeder, K. Sekiguchi, J. Phys. Chem. A, 2005, 109, 10824. 63,64

[128] A. Charvat, J. Aßmann, B. Abel, D. Schwarzer, K. Henning, K. Luther, J. Troe, Phys. Chem. Chem. Phys., 2001, 3, 2230. 64

[129] M. Tamres, in: R. Foster, Hg., Molecular Complexes, vol. 1, Paul Elek (Scientific Books) Ltd., London, 1973 49-116, 49-116. 65

[130] M. Tamres, in: R. Foster, Hg., Molecular Associations, vol. 2, Academic Press Inc. (London) Ltd., London, 1979 331-456, 331-456. 65

[131] K. Dahl, R. Biswas, M. Maroncelli, J. Phys. Chem. B, 2003, 107, 7838. 65

[132] G. T. Evans, D. Kivelson, J. Chem. Phys., 1986, 84, 385. 65

[133] J. A. Seetula, Phys. Chem. Chem. Phys., 2002, 4, 455. 66

[134] Z. Kisiel, L. Pszcölkowski, W. Caminati, P. Favero, J. Chem. Phys., 1996, 105, 1778. 66

[135] G. Käb, V. S. Vikhrenko, Phys. Chem. Chem. Phys., 2001, 3, 2223. 66

[136] K. Sekiguchi, A. Shimojima, O. Kajimoto, Chem. Phys. Lett., 2002, 356, 84. 71, 81

[137] J. Troe, J. Chem. Phys., 1982, 77, 3485. 76

[138] D. Schwarzer, J. Troe, M. Votsmeier, M. Zerezke, J. Chem. Phys., 1996, 105, 3121. 76

[139] D. Schwarzer, J. Troe, M. Zerezke, J. Phys. Chem. A, 1998, 102, 4207. 76

[140] C. Böttcher, Theory of Electric Polarisation, Elsevier, 1973. 78

[141] N. Mataga, T. Kubota, Molecular Interactions and Electronic Spectra, Marcel Dekker, New York, 1970. 78

[142] J. Schroeder, in: J. H. Moore, N. D. Spencer, Hgs., Encyclopedia of Chemical Physics and Physical Chemistry, vol. 1, Institute of Physics Publishing, 2001 711-743, 711743. 78

[143] J. Lewis, R. Biswas, A. Robinson, M. Maroncelli, J. Phys. Chem. B, 2001, 105, 3306. 78

[144] T. A. Litovitz, E. H. Carnevale, P. A. Kendall, J. Chem. Phys., 1957, 26, 465.79

[145] S. H. Northrup, J. T. Hynes, J. Chem. Phys., 1979, 71, 871.79

[146] S. H. Northrup, J. T. Hynes, J. Chem. Phys., 1979, 71, 884. 79

[147] M. Quack, Nuovo Cim., 1981, 63B, 358. 80 
[148] K. Sekiguchi, A. Shimojima, O. Kajimoto, Chem. Phys. Lett., 2003, 370, 303.81

[149] X. Zheng, W.-H. Fang, D. L. Phillips, J. Chem. Phys., 2000, 113, 10934. 82

[150] F. Duschek, M. Schmitt, P. Vogt, A. Materny, W. Kiefer, J. Raman Spec., 1997, 28, 445. 82 


\section{Lebenslauf}

Am 9.07.1976 wurde ich als Sohn von Friedrich Zerbs und Ehefrau Gisela, geb. Aust in Fulda geboren.

Von 1983 bis 1987 besuchte ich die Don-Bosco Grundschule in Künzell. Von 1987 bis 1996 besuchte ich das Freiherr-vom-Stein Gymnasium in Fulda, wo ich die Allgemeine Hochschulreife im Juni 1996 erhielt.

Vom WS 1996/97 bis zum SS 1997 studierte ich Bauingenieurwesen an der Bauhaus Universität Weimar. Anschließend wechselte ich an die Georg-August Universität Göttingen, wo ich im WS 1997/98 mit dem Studium der Chemie begann. Unter Anleitung von Prof. Jürgen Troe fertigte ich im Institut für Physikalische Chemie eine Diplomarbeit mit dem Titel „Untersuchungen zur Ultrakurzzeitdynamik von intramolekularen Ladungsübertragungsprozessen von Aminobenzonitrilderivaten in Lösung" an und schloss das Studium im Februar 2002 mit der Diplomprüfung ab. Vom WS 2002/2003 bis zum SS 2004 absolvierte ich das Fernstudium Lasertechnik an der Friedrich-Schiller-Universität Jena.

Als wissenschaftlicher Mitarbeiter des Institutes für Physikalische Chemie beschäftigte ich mich mit Femtosekundenspektroskopie von unimolekularen Reaktionen in Lösung. Meine Arbeit beinhaltet die Photoisomerisierung von all-trans-1,4-Diphenylbutadien, ultraschneller elektronischer Energietransfer in bichromophorischen Molekülen (in Zusammenarbeit mit Prof. Vlastimil Fidler, Prag) und die Photoisomerisierung von Dihalomethanen in überkritischen Fluiden als Hauptthema. Als Ergebnis dieser Forschung entstand die vorliegende Dissertation. 